


\title{
Molecular DNA Sensors to Measure Distribution of \\ Cytoskeletal Forces
}

\author{
Dissertation \\ for the award of the degree \\ "Doctor rerum naturalium" \\ of the Georg-August-Universität Göttingen \\ within the doctoral program \\ Göttingen Graduate School for Neurosciences, \\ Biophysics, and Molecular Biosciences (GGNB) \\ of the Georg-August University \\ School of Science (GAUSS)
}

submitted by

Christina Jayachandran

from Chennai (India)

Göttingen, 2019 



\section{Thesis Committee:}

Prof. Dr. Christoph F. Schmidt

$3^{\text {rd }}$ Institute of Physics - Biophysics

Georg-August-Universität, Göttingen

Dr. Florian Rehfeldt

$3^{\text {rd }}$ Institute of Physics - Biophysics

Georg-August-Universität, Göttingen

Prof. Dr. Fred Wouters

Molecular and Cellular Systems Lab, Institute for Neuropathology

University Medical Center, Göttingen

Prof. Dr. Max Wardetzky

Institute of Numerical and Applied Mathematics,

Georg-August-Universität, Göttingen 


\section{Members of the Examination Board:}

$1^{\text {st }}$ Reviewer: Prof. Dr. Christoph F. Schmidt

$3^{\text {rd }}$ Institute of Physics - Biophysics

Georg-August-Universität, Göttingen

$2^{\text {nd }}$ Reviewer: Prof. Dr. Fred Wouters

Institute for Neuropathology, Molecular and Cellular Systems Lab

University Medical Center, Göttingen

\section{Additional members of the Examination Board:}

Dr. Florian Rehfeldt

$3^{\text {rd }}$ Institute of Physics - Biophysics,

Georg-August-Universität, Göttingen

Prof. Dr. Max Wardetzky

Institute of Numerical and Applied Mathematics,

Georg-August-Universität, Göttingen

Prof. Dr. Stefan Klumpp

Institute for Dynamic Complex Systems

Georg-August-Universität, Göttingen

Dr. Andreas Neef

Centre for Biostructural Imaging and Neurodegeneration (BIN)

Max Planck Institut for Experimental Medicine, Göttingen

\section{Date of Oral Examination:}




\section{Contents}

\begin{tabular}{ll}
\hline 1. Introduction 1 & 1
\end{tabular}

1.1. Outline . . . . . . . . . . . . . . . . . . 5

\begin{tabular}{ll}
\hline 2. Methods & 7
\end{tabular}

2.1. Spectrometer - Emission Spectrum . . . . . . . . . . . . . . . 7

2.2. Force Spectroscopy and Imaging . . . . . . . . . . . . . . . . 8

2.2.1. Confocal Microscopy . . . . . . . . . . . . . . . . 8

2.2.2. Förster Resonance Energy Transfer (FRET) . . . . . . . . . . . 8

2.2.3. $\quad$ Fluorescence Lifetime Imaging Microscopy (FLIM) _ . . . . . 10

2.2.3.1. Time Correlated Single Photon Counting (TCSPC) . 11

2.3. Macrorheology . . . . . . . . . . . . . . . . . . . 12

2.3.1. Viscoelastic Measurements . . . . . . . . . . . . . . . . . 14

2.4. Microrheology . . . . . . . . . . . . . . . . . . . 17

2.4.1. Single Particle Passive Microrheology (1PMR) . . . . . . . . 17

2.4.2. $\quad$ Principle of Optical Trapping . . . . . . . . . . . . . . . . . 19

2.4.3. Detection via Back Focal Plane Interferometry . . . . . . . . . 19

2.4.4. Calibration of Trap Stiffness . . . . . . . . . . . . . . 21

2.4.5. Instrumentation of Optical Trap . . . . . . . . . . 22 
Contents

\begin{tabular}{ll}
\hline 3. Force Sensor Characterization 25 & 25
\end{tabular}

3.1. Sensor Design . . . . . . . . . . . . . . . . . . . 25

3.2. Spectrometer: Bulk Fluorescence Intensity . . . . . . . . . . . . . 27

3.2.1. Assembly and Conformation of Sensor via Bulk Fluorescence Measurements . . . . . . . . . . . . . 27

3.2.2. Hairpin Opening: Binding Energy . . . . . . . . . . . . . . 28

3.2.3. Bulk Fluorescence: DNA Sensors versus Controls . . . . . . . 30

3.2.4. Structural Quenching: Reduction in Fluorescence Intensities 30

3.3. Sensor Characterization via Fluorescence Lifetime Imaging Microscopy

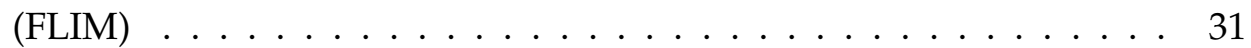

3.3.1. Experimental Procedure . . . . . . . . . . . . . . . 32

3.3.2. FLIM: Structural Quenching of Sensors . . . . . . . . . . . 32

3.3.3. Finding the Efficient Quenching stoichiometry . . . . . . . . . 34

3.3.4. FLIM on Quenched and Opened Sensors . . . . . . . . . . . 37

3.3.5. FLIM on Controls in Closed and Open Position . . . . . . . . 38

3.3.6. PCR Annealing of Sensors Distorts Quenching . . . . . . . . 39

3.3.7. FRET Efficiency of DNA Sensors . . . . . . . . . . . . . . 42

3.3.8. Discussion . . . . . . . . . . . . . . . . 43

4. DNA Sensors in In Vitro Actin Network \& in Cellular Actin 45

4.1. DNA Sensors Crosslinked to Actin (In Vitro) . . . . . . . . . . . . . . 45

4.1.1. Spectrometer: Bulk Fluorescence of Sensors Crosslinked to

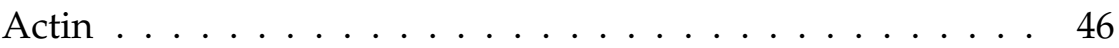

4.1.2. Confocal Laser Scan: Quenching and Fluorescence of Sensors across the Network . . . . . . . . . . . . . . . . 48 48 
4.1.3. Fluorescence Lifetime Imaging Microscopy (FLIM) on ActinDNA Sensor Network . . . . . . . . . . . . . . . . 50

4.1.3.1. FLIM on Sparsely Crosslinked Actin-DNA Sensor Network $(R=0.01) \ldots \ldots \ldots \ldots \ldots$

4.1.3.2. $\quad$ FLIM on Densely Crosslinked Actin-DNA Sensor Network $(R=0.1)$

4.2. DNA Sensors in Fibroblast's Actin . . . . . . . . . . . . . . . . . . . 59

4.2.1. DNA Sensors in 3T3 Fibroblasts . . . . . . . . . . . . . . 59

4.2.2. Controls in 3T3 Fibroblasts . . . . . . . . . . . . . . . . . 62

4.2.3. Discussion . . . . . . . . . . . . . . . . . . . . 64

5. Mechanics of Actin-DNA Sensor Networks 67

5.1. Introduction . . . . . . . . . . . . . . . . . . 67

5.2. Macrorheology of Actin and Actin-DNA Sensor Networks . . . . . . 68

5.2.1. Gelation Kinetics: Elastic Shear Modulus of Networks . . . . 69

5.2.2. Bulk Frequency Response of Networks . . . . . . . . . . . . . 71

5.3. Imaging of Actin-DNA Sensor Networks Morphology . . . . . . . . 73

5.4. Optical Trap: Microrheology of Actin and Actin-DNA Sensor Network 75

5.4.1. Laser Power Optimization . . . . . . . . . . . . . . . . . . . . 75

5.4.2. Frequency Response of Densely Crosslinked Actin-DNA Sensor Network $(R=0.1) \ldots \ldots \ldots \ldots$. . . . . . . . . . 81

5.4.3. Discussion . . . . . . . . . . . . . . . . . . . 84

6. Summary \& Outlook 87

\begin{tabular}{ll}
\hline A. Appendix - Protocols & 91
\end{tabular}

A.1. Biochemical Protocols . . . . . . . . . . . . . . . . . . . . . . . . 91

A.1.1. KOH Cleaning . . . . . . . . . . . . . . . . . . . . . 92

A.1.2. Preparation of Chambers for Actin Networks . . . . . . . . 92 
A.1.3. Actin and Actin-DNA Sensor Network Sample Preparation . 93

A.1.4. Preparation of Sensor Strands F and Q . . . . . . . . . . 93

A.1.5. VALAP Recipe . . . . . . . . . . . . . . . . . . . . 94

A.1.6. PCR Annealing and PAGE Electrophoresis . . . . . . . . . . 94

A.2. Cell Culture Protocols . . . . . . . . . . . . . . . . . . . . . . . . . 98

A.2.1. Passaging of Cells . . . . . . . . . . . . . . . 98

A.2.2. Double Transfection of Cells . . . . . . . . . . . . . . . . . . 99

A.3. Protein Expression and Purification . . . . . . . . . . . . 101

\begin{tabular}{lr}
\hline B. Supporting Information & 105
\end{tabular}

B.1. Spectrometer: Bulk Fluorescence of Sensor and Controls across Actin $(1: 1: 1$ - F:H:Q) $\ldots \ldots \ldots \ldots \ldots \ldots$

B.2. Confocal Laser Scan: Quenching and Fluorescence of Sensors across the Network (1:1:1 - F:H:Q) ～. . . . . . . . . . . . . . . . . . . 107

B.3. FLIM: Actin-DNA Sensor Network Equal stoichiometry (1:1:1-F:H:Q) 108 B.3.1. FLIM: Sparsely Crosslinked Network $R=0.01$. . . . . . . . 108 B.3.2. FLIM: Densely Crosslinked Network $R=0.1$. . . . . . . . . 110

B.4. FLIM: DNA sensors in Fibroblasts . . . . . . . . . . . . . . . . 112

\begin{tabular}{ll}
\hline Bibliography & 113
\end{tabular}

\begin{tabular}{ll}
\hline List of Figures & 127
\end{tabular}

\begin{tabular}{ll}
\hline List of Tables & 131
\end{tabular}

\begin{tabular}{ll}
\hline Terms and Abbreviations & 133
\end{tabular} 


\section{Abstract}

Actin, a major cytoskeletal biopolymer in eukaryotic cells, is crosslinked into networks of filaments and bundles. These networks are largely responsible for the maintenance of cellular shape, rigidity, and mechanical stability. Other assemblies of actin are involved in a myriad of cellular processes, such as cell migration, division, intracellular transport, and morphogenesis. In these processes, the spatial and temporal regulation of the network structure, their dynamics, and force generation due to myosin motors are crucial. Experimentally, one of the challenges is to measure force transmission across such networks, which is vital to properly understand the function, failure, and repair mechanisms beyond the linear regime. To measure forces across the cytoskeletal network, we have developed a FRET-based, reversible DNA force sensor. We employ these DNA constructs as flexible crosslinkers across semiflexible actin, thereby reconstituting model networks of cytoskeletal structures. Characterization of the rheology and frequency response of these model actin-DNA sensor networks is performed via a macrorheometer and also by utilizing a large bandwidth, high-resolution microrheology set up. DNA force sensors are crosslinked in vitro with actin filaments in order to map force distributions and stress relaxations in the resulting network. We characterize the DNA force sensor in solution and across actin networks through fluorescence lifetime imaging microscopy (FLIM) measurements. From these results, we estimate the FRET efficiency of our DNA sensor. We also test DNA sensors in a cellular environment and describe its preliminary results. 



\section{1 \\ Introduction}

Cells, the fundamental unit of life, generate and transmit forces to their environment. The internally created forces are borne by the cytoskeleton. It is a fibrous polymer scaffold that is comprised of the actin microfilaments, microtubules and intermediate filaments and molecular motors - primarily myosin and kinesin (Fig. 1.1). These scaffold structures support cellular rigidity, function and act as mediator of mechanical forces inside the cell [131]. Extensive research has been done to elucidate the mechanical role of these structures [38], [21], [13], [109], [53], [124] indicating that each play a significant function in aiding the cell to resist and recover from mechanical forces. Molecular motors create tension along cytoskeletal filaments (that are anchored to the surrounding via so-called focal adhesions) by actively contracting neighboring filaments [29], [115]. For example the cross-bridging of actin with myosin leads to muscle contractions [18] and the propulsive force created by actin polymerization drives cell migration [105]. Since the focus of this thesis will be on the actin cytsokeleton and networks, the next section will elaborate on them further.

F-actin (filamentous actin) is a semiflexible polymer composed of monomers of $42 \mathrm{kDa}$ globular actin (G-actin) that assembles into a double stranded, helical structure with $7 \mathrm{~nm}$ diameter. Inside the cell, a crosslinked polymeric actin network forms the cellular cortex. It acts as a mechanical barrier [24], transmits internal forces [55] and contributes to cellular elasticity [88],[56],[30]. 


\section{\begin{tabular}{l|l} 
Chapter 1 & INTRODUCTION
\end{tabular}}

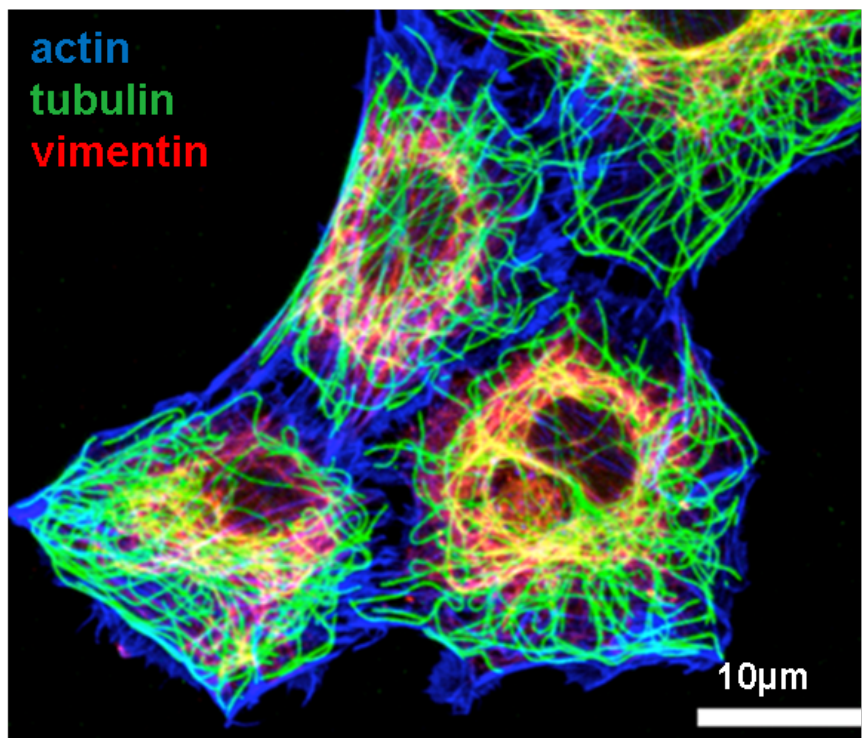

Figure 1.1.: Cytoskeletal components of a cell. A fluorescent image of a cell with the three cytoskeletal filaments - actin, microtubules (tubulin) and intermediate filament (vimentin). Image is reproduced with permission from [98] which was originally obtained from Rosmarie Sütterlin and Ueli Aebi, Biozentrum, University of Basel.

A few hundred nanometers thick cortical shell [89],[27], consists of branched actin networks, that is nucleated by actin binding protein (ABP) Arp2/3 that localizes to the cortical actin network and is responsible for cortex formation [72]. The different types of actin networks present inside the cell are as follows. F-actin bundles are located in lamellopodial and filopodia. Linear arrays of actin filaments that constitute the lamellum run behind the lamellopodium that gets organized into longitudinal contractile bundles called stress fibers. These anchor the cell to a substrate via focal adhesions (Fig 1.2] [85], [86].

To understand the mechanical contribution of these actin networks, they are reconsituted in vitro with the help of actin binding proteins (ABPs). An example of such an ABP in the cortex is the large and flexible filamin, that crosslinks actin filaments into orthogonal networks [117]. When reconsituted in vitro, these networks show a moderate linear elasticity but when a pre-stress is applied they exhibit a pronounced non-linear elastic behavior [39]. Other ABP's are $\alpha$-actinin, fascin, and scruin which organize actin into bundled networks due to their smaller size and produce stiffer networks than filamin. Hence by varying the crosslinking density, these networks can be mechanically tuned for their stiffnesses [127], [64] , [5], [40].

\footnotetext{
${ }^{\mathrm{i}}$ membrane protrusions
} 


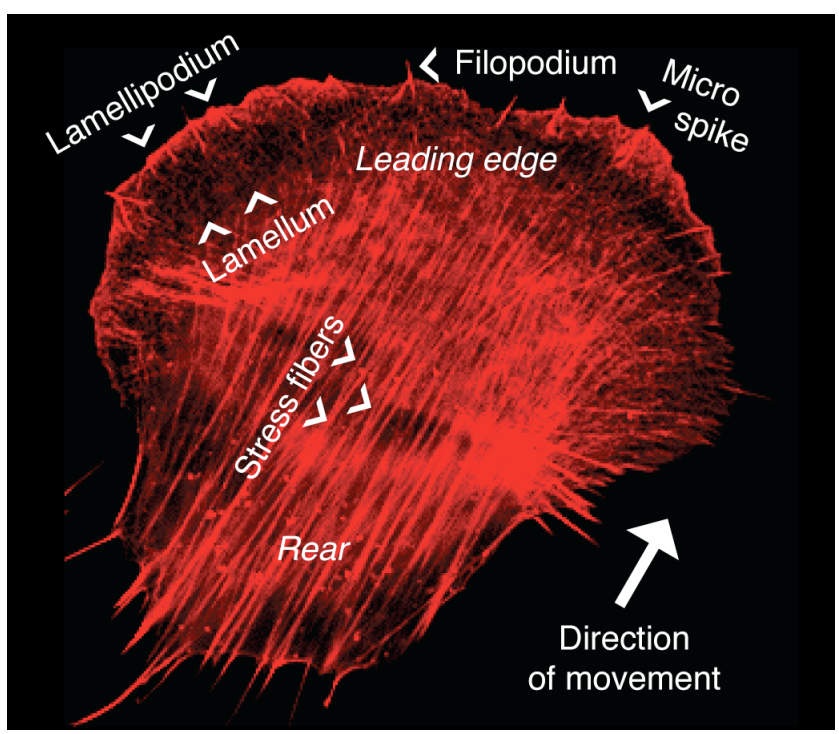

Figure 1.2.: Actin structures in an adherent cell. The different actin architectures inside a fixed cell of a primary chick embryo fibroblast. The filopodia and microspikes are seen originating from the lamellipodium. Lamellum is present beneath the lamellopodia followed by the presence of contractile bundles of actin stress fibers found at the rear end of the cell. Figure is adapted after [86].

A major question is how are stresses transmitted in such networks. To address this, we have developed molecular tension sensors (DNA based) that crosslink actin filaments into networks and simultaneously might be able to probe tension distribution upon external force application. Physical forces that exists inside the cell ranges from a picoNewton $(\mathrm{pN})$ to several hundred's of nanoNewton $(\mathrm{nN})$. For example, actin polymerization occurs at $1 \mathrm{pN} \mathrm{[34]} \mathrm{and} \mathrm{the} \mathrm{pulling} \mathrm{force} \mathrm{between} \mathrm{cells} \mathrm{are} \mathrm{around} 100 \mathrm{nN}$ [31], [95].

To measure these forces at the molecular scale, mechanical instruments have been employed in the past. They can be classified into two categories. The first category is single molecule force spectroscopy which includes atomic force microscopy - AFM [48], optical trap (OT) [10], magnetic tweezers [114] or biomembrane force probes [65]. In these techniques, forces on single molecules are measured as well as force can be exerted on them to observe their response. In the second category, forces that are externally transmitted are measured. For example, force exerted by cells on their underlying substrate are quantified. Traction force microscopy (TFM) [118] or micropost array detectors are the commonly used methods to measure these external transmitted forces [120].

An ideal approach in these measurements would be, to have a single molecule force resolution alongside observation of individual molecule interaction. For this purpose, molecular force sensors (MFSs) had been developed. They can be directly integrated into 


\section{\begin{tabular}{l|l} 
Chapter 1 & INTRODUCTION
\end{tabular}}

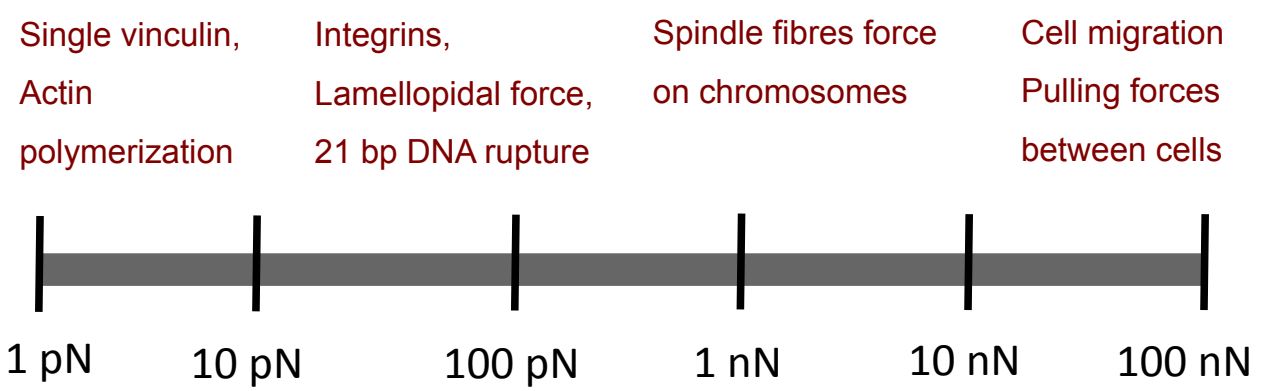

Figure 1.3.: Physical forces at the molecular scale. Molecular physical forces that exists across various biological structures and during cellular processes is sketched. It ranges from a piconewton to hundred's of nanonewton.

the structure to be probed. MFSs are designed with an elastic extendable molecular spring flanked by fluorophore pairs based on Förster resonance energy transfer (FRET) such that any structural change due to force application will result in a fluorescence readout. The other ends of MFSs can be modified to attach to the force component of interest. In this way, high throughput of TFM can be combined along with the piconewton sensitivity of MFSs.

Two classes of MFSs exists, the protein based and DNA based MFSs. Protein MFSs uses protein as the elastic extendable linker between two fluorescent probes. They are genetically integrated into the protein or cell structure to be probed. These measure intracellular forces of around 2-6 pN [37], [83], [46], [12], [23], [132]. The first genetically encoded protein MFS's measured stresses in proteins such as $\alpha$-actinin, filamin A and non-erythryoid spectrin by a direct insertion into these proteins [84].

The second new class of tension sensing probes are the DNA force sensors which have been recently developed. It employs DNA hairpins that act as a 'switch' element, i.e. they open at a threshold force and closes when forces cease [19], [138]. The design of DNA sensors is based on molecular beacons (MBs) which are single stranded nucleic acid probes. They are composed of 3 elements, a stem, a loop and a fluorophore/quencher pair. MBs function by a on/off mechanism depending on their conformation. Hairpins (MBs) are employed in DNA sensors since their folding energies, kinetic rates and transition state distance can be modified, eventually their dynamics [137], [125]. DNA sensors offers the feasibility of having a varied force range by simpling tuning the Guanine-Cytosine (GC) content, the stem length and loop sizes [138]. Also, by varying the force application geometry, a broad force range can be detected for a given same length, sequence and thermal stability. The tension gauge thether (TGT) is one such, with 21 base pairs (bp) that can be unzipped at $12 \mathrm{pN}$ while the same tether when sheared, ruptures at $56 \mathrm{pN}$ [132]. Since the length between two base pairs in a DNA $(0.33 \mathrm{~nm})$ is known, it gives the feasibility of designing structures with different lengths. 
Single stranded [19] and double stranded DNA sensors were used to probe forces across focal adhesions [138]. Single stranded DNA force sensor can sense forces about $6 \mathrm{pN}$ [113]. The first force information on an individual T-cell receptor antigen was performed using a DNA hairpin probe [71]. TGT were used either as a single strand or double strands to quantify forces in Notch-receptor mechanics [26], B-cell receptor activation forces [130], T-cell receptors [66], E-cadherin and P-selectin mechanics [133].

The added advantage of a DNA force sensor is that the problem of ensemble averaging is overcome by using a one-component DNA sensor (e.g MBs). This design has a high FRET (ON) and low FRET (OFF). Thus, the the fraction of sensors in either of the states can be directly calculated from the FRET efficiency [45]. Also the flexibilty of having a vast array of designs, thereby a varied force range, makes it a desirable choice over protein force sensors which measure low forces of approximately 2-6 pN. The downside of utilizing DNA tension sensors is that directional information of forces is unavailable [45]. Also, the unfolding of DNA and protein structures are dependent on loading rate. Therefore in a DNA sensor, secondary structures can unfold in the presence of small forces (1-5 $\mathrm{pN}$ ) [67, provided their length are long. Our design of DNA force sensor is similar to MBs that utilizes a hairpin by having a on \& off mechanism that gives a high \& low FRET. Also our DNA sensors are quite short (hairpin stem and loop $=24 \mathrm{bp}$ ) for any secondary structures to be present.

\subsection{Outline}

Chapter 2 describes the various methodologies used in this work. This thesis employs techniques from the field of spectroscopy, imaging and mechanics. Hence the theory behind each method is discussed. On the force spectroscopy and imaging side, the method of FLIM (Fluorescenece lifetime imaging microscopy), spectrometer and confocal laser scanning microscopy is discussed. Macro- and Micorrheology principles, their instrumentation and calibration are described in detail.

Chapter 3 describes the entire characterization of our molecular DNA sensor. The efficient functionality of our sensors is tested in a spectrometer. Lifetimes of sensors were determined in two different buffer solutions by performing FLIM measurements. In this chapter we also introduce the control probes termed as "controls" that we had developed for our DNA sensors and had also tested their performance in a spectrometer. Their lifetimes were then determined through FLIM. Finally, the FRET efficiency of our sensors is estimated from lifetimes determined via FLIM. 


\section{\begin{tabular}{l|l} 
Chapter 1 & INTRODUCTION
\end{tabular}}

In chapter 4 the attachment of sensors to actin in vitro and inside cells is shown. We characterize the quenched and fluorescence state of the sensor while it is crosslinked in an actin network. The lifetime of sensors in the actin network in these two states is determined. We then introduce our sensors into a live cellular environment. 3T3 fibroblasts were used for this to test the working of molecular DNA sensors by investigating their lifetimes inside the cell. As a control experiment, we also introduce our controls into the actin network, into cells and measured their lifetimes.

Chapter 5 illustrates the mechanics of the model actin-DNA sensor networks. We use macrorheology and microrheological approaches to probe the linear elastic behavior and the frequency response of actin-DNA sensor networks. The DNA sensor concentration in the networks is varied to obtain crosslinked networks with different mechanical stiffness. This effect of crosslinking is proved via the gelation kinetics experiment in a macrorheometer. The local viscoelastic properties of the actin-sensor network was investigated through microrheology. Prior to this experiment, optimization experiments in microrheology were done in which an appropriate laser power and sampling frequency was chosen to study the frequency response of actin-DNA sensor network.

Chapter 6 summarizes this work, and briefs upon the potential developments and modifications that can be done for our design of molecular DNA sensor. Protocols, materials and supporting information are given in appendices $A, B$. 


\section{2 \\ Methods}

\subsection{Spectrometer - Emission Spectrum}

The efficiency of our DNA molecular force sensor, which has the FRET pair Alexa 488 and a quencher, was tested in a spectrometer by measuring the quenched and fluorescence intensities via an emission spectrum. Hence a brief illustration of fluorescence, spectrometer and its instrumentation will be given. A fluorescent molecule when illuminated with light, absorbs energy and reaches the excited state. After losing some energy due to vibrational collision, it returns to the ground state resulting in an emission of photons that is termed as fluorescence. An emission spectrum thus is a plot of wavelength against the emission intensity when the fluorescent molecule is excited with a specific wavelength [60].

In this thesis, a single beam spectrometer was used, where the components are arranged in a single beam sequence. The light beam from a Xenon flash lamp was passed through a stepper meter controlled monochromator (diffraction grated - ion etched concave holographic gratings). The monochromator is placed between the source and sample to analyze one wavelength at a time. Hence the reference measurement (blank sample) has to be measured separately from the test sample. An AMINCO-Bowman Series $2 \mathrm{Lu}-$ minescence Spectrometer (Thermo Electron Scientific Instruments Corporation, Madison, WI 53711,USA) was used for all the emission spectrums recorded for this work. The emission intensity scan was performed with the following settings. Excitation wavelength - 


\section{\begin{tabular}{l|l} 
Chapter 2 & METHODS
\end{tabular}}

$488 \mathrm{~nm}$, Emission wavelength - $520 \mathrm{~nm}$, Bandpass - 1. Emission scan range : $490-600 \mathrm{~nm}$. Cuvette containing the sample solution was placed in line with the incident beam and the detector was at right angles to the incident beam.

\subsection{Force Spectroscopy and Imaging}

Confocal laser scanning microscopy was used for quantitative and qualitative imaging of actin-DNA sensor networks. Likewise fluorescence lifetime measurements were done to determine the lifetimes of the quenched and fluorescent sensors. Hence the methodology behind these two techniques will be described here in short.

\subsubsection{Confocal Microscopy}

Confocal laser scanning microscopy was used to quantify the intensity of sensors in both quenched and fluorescent state while crosslinked to actin filaments. Qualitative imaging of these actin-DNA sensors networks were done via confocal scans to visualize their morphology across their z-height. It was achieved by staining actin with Atto 647N Phalloidin, in which phalloidin intercalates actin filaments. A Leica TCS SP5 (Leica Microsystems CMS GmbH, Mannheim, Germany) was used for this purpose with a 63X oil immersion objective $(\mathrm{NA}=1.4)$. DNA sensors fluorescence intensity in actin networks were quantified with $488 \mathrm{~nm}$ wavelength. The microstructure of the actin-DNA sensor networks were imaged at $647 \mathrm{~nm}$. A white light laser was used as the illumination source with $20 \%$ intensity for the sensor channel (excitation wavelength - $488 \mathrm{~nm}$ ) and $22 \%$ for the actin network imaging channel (excitation wavelength - $647 \mathrm{~nm}$ ). Image acquisition was performed by a 16 line scan in a bidirectional manner with pixel dimensions of 1024 X 1024 . This gives a field of view of $100 \mu \mathrm{m} \times 100 \mu \mathrm{m}$. A zoom factor of 2.5 was used. A pinhole size of Airy $1(95.4 \mu \mathrm{m})$ was used for Z- stack imaging of networks with a step size of $3 \mu \mathrm{m}$.

\subsubsection{Förster Resonance Energy Transfer (FRET)}

Also known as fluorescence energy transfer, was first described by Theodor Förster in 1948 [35]. FRET is a mechanism of non-radiative energy transfer that takes place between two fluorophores when they are in close vicinity with each other. In this process, the excited state donor fluorophore transfers part of its energy to a second, non-excited, fluorophore (acceptor) in a radiation-less manner (no emission of photons from the donor takes place) due to a long range dipole-dipole interaction (Fig. 2.14). The theory behind this interaction is, that the excited donor is considered as an oscillating dipole that can transfer its energy to a second dipole which resonates at the same frequency. Hence the 


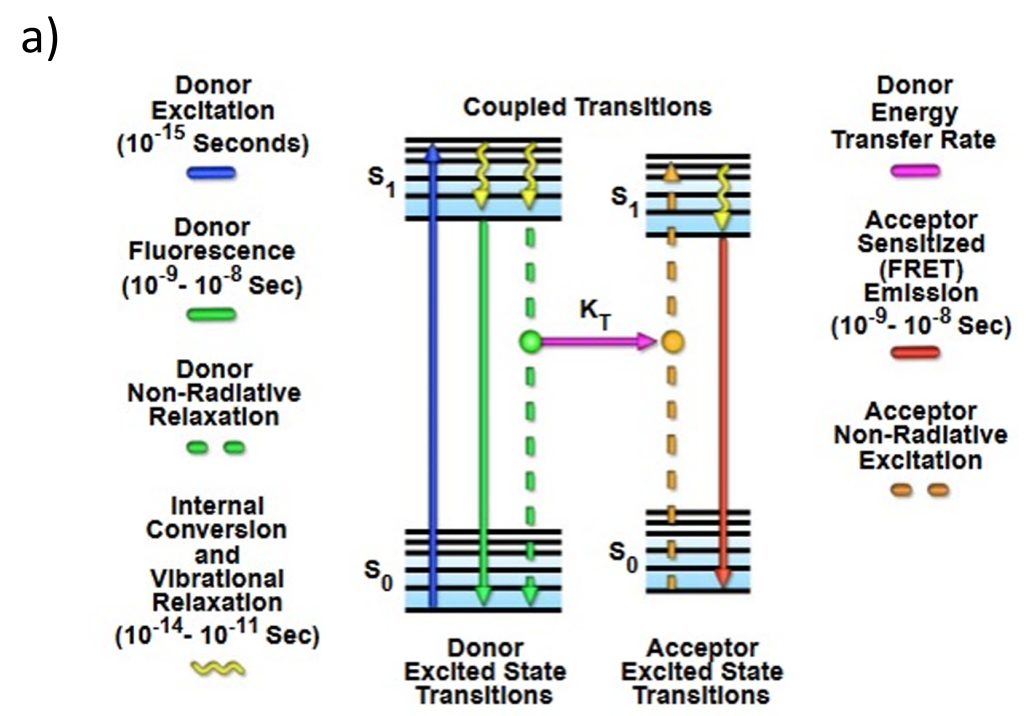

b)

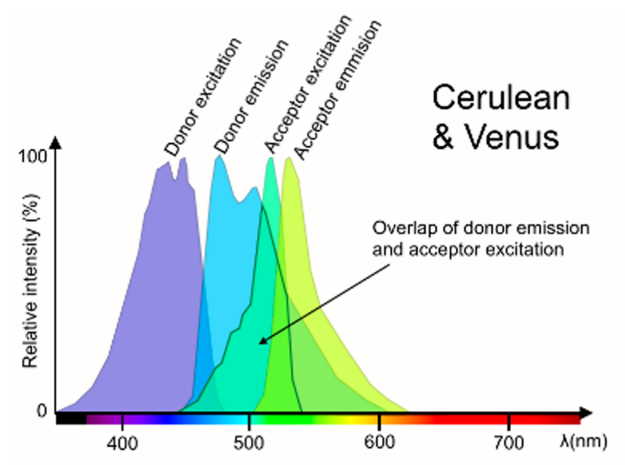

c)

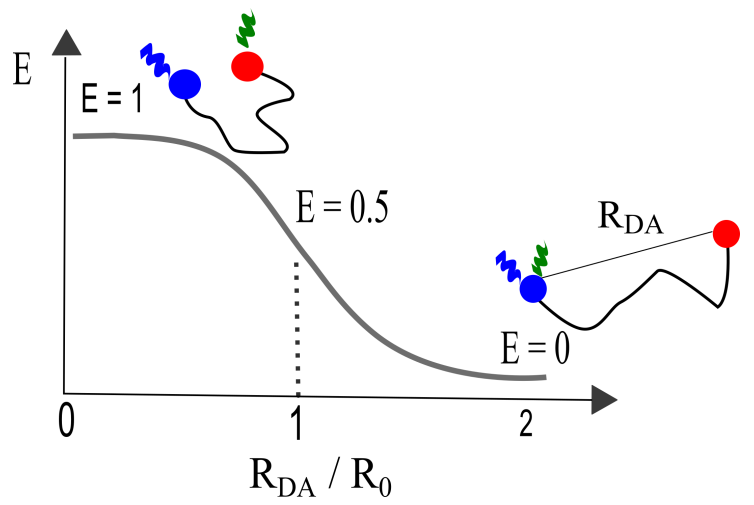

Figure 2.1.: FRET mechanism. a) Jablonski diagram illustrating the phenomenon of FRET. Upon light absorption, a fluorescent molecule gets excited to higher electronic states and emits fluorescence when returning to the ground state (solid arrows). Dashed arrow (green) represents the energy relaxation which is taken up by the second fluorophore (yellow dashed arrow) at the nearby vicinity. It then gets excited to higher energy state which upon its return to the ground state, emits fluorescence. b) The spectral overlap integral for a FRET pair, cerulean and venus. c) Scheme of FRET efficiency $(E)$. When fluorophores are apart $(E=0)$ and when near to each other $(E=1) . E=0.5$ represents the Förster distance $R_{0}$ at which $50 \%$ FRET has taken place. a) adapted from [96, b taken from [50], c) redrawn after [4].

term resonance energy transfer, as it is analogous to the behavior of coupled oscillators [35].

Certain pre-requirements are needed for FRET to occur. The two fluorophores (donor and acceptor) should be at a distance of 1-10 nm, where the energy transfer between them 


\section{\begin{tabular}{l|l} 
Chapter 2 & METHODS
\end{tabular}}

varies as the sixth power of its distance. The emission spectra of the donor fluorophore should overlap with the absorption spectrum of the acceptor (second fluorophore) which is given as the spectral overlap integral in Eqn. 2.1. An example of the overlap integral is shown in (Fig. 2.1p) for a cerulean-venus FRET pair. Their dipole vectors should be oriented relative to each other for a high FRET efficiency. While designing FRET pairs, the second fluorophore (acceptor) can also be a dark absorber or quencher where it diminishes the fluorescence of the first fluorophore (donor). Two types of FRET can happen, heteroFRET and homo-FRET. When it occurs between two fluorophores it is hetero-FRET and when it occurs within the same fluorophore due to a small stokes shift of few nanometeres it is called homo-FRET. The spectral overlap integral (J) is given as

$$
J=\int_{0}^{\infty} F_{D}(\lambda) \epsilon_{A}(\lambda)(\lambda)^{4} d \lambda
$$

$\mathrm{F}_{D}$ - donor fluorescence, $\epsilon_{A}$ - maximum molar extinction coefficient. The FRET efficiency (E) varies inversely as the sixth power of the distance between the two fluorophores. It is written as,

$$
E=\frac{1}{1+\left(\frac{R_{D A}}{R_{0}}\right)^{6}}
$$

where $\mathrm{R}_{D A}$ is the distance between the donor and acceptor fluorophore, $\mathrm{R}_{0}$ is the Förster radius distance. It describes the characteristic distance at which FRET efficiency is $50 \%$ $(E=0.5)$ or in other words, $50 \%$ of donor excitation events lead to FRET. Fig. 2.1p represents the efficiency of the FRET process [99], [68], [50].

\subsubsection{Fluorescence Lifetime Imaging Microscopy (FLIM)}

When two fluorophores of a FRET pair fluoresce, their sensitised emission can be determined by radiometeric techniques which characterizes the FRET efficiency. However, when dark quenchers are used as acceptors, as in our DNA sensors, their emission cannot be quantified, in which case the donor lifetime is measured in a fluorescence lifetime imaging microscopy (FLIM) experiment. Fluorescence lifetime is the average lifetime that the fluorophore spends in the excited state after absorbing photons, before returning to the ground state. This lifetime is in the order of several nanoseconds $\left(10^{-8}-10^{-9} \mathrm{~s}\right)$. But in the presence of an acceptor, the donor lifetime gets shortened due to FRET. Hence fluorescence lifetime is a direct indication of an energy transfer process from the first fluorophore to the nearby second fluorophore or to its local environment [60].

In a FLIM-FRET experiment, these changes in the donor fluorescence lifetime due to FRET are measured. Lifetimes are independent of the concentration, are not altered by laser or detector gain settings [60], [123] and are the best characterization when dark 
quenchers are used as one of the FRET pair. In this thesis, lifetime measurements are therefore utilized for our DNA sensors to estimate its FRET efficiency which is the fraction of photons transferred from donor (fluorophore) to acceptor (quencher) and is given as

$$
E=1-\frac{\tau_{D A}}{\tau_{D}}
$$

$\tau_{D A}$ - Donor lifetime in the presence of acceptor, $\tau_{D}$ - Donor lifetime in absence of acceptor.

Lifetime measurements can be made either in the time or frequency domain which differs in the signal recording technique [17].

(a) Time-Domain technique: Here a short sharp laser pulse is given to excite fluorophores. The decay of photons as fluorescence is then recorded directly. This is performed mostly on confocal laser scanning microscopes.

(b) Frequency-Domain technique: Here a phase shift is employed between the pulsed excitation and the sample emission. This measurement is done in wide field fluorescence microscopes [100].

\subsubsection{Time Correlated Single Photon Counting (TCSPC)}

\section{Principle \& Instrumentation}

The time domain experiment is done via TCSPC. It is based on single photon counting that is combined with image scanning methods [100]. It is a highly sensitive technique as it counts every single photon that arrives at the detector. Besides it also gives a high accuracy when multi-exponential decays are involved. The key principle here is the precise measurement of the time between a given laser pulse of excitation and the arrival of the first emitted photon at the detector that gives the fluorophore's lifetime.

In brief, short intense laser pulses are repetitively given to excite the photons. The time difference between the excitation and emission is measured at the level of single photons by electronics which act as a stopwatch. Hence a start and stop signal is needed. The 'start' signal is the time of laser pulse excitation of donor fluorophore which is provided by electronics that steer either the laser pulse or the photodiode. The 'stop' signal is the time at which a single photon arrives at the detector. The time between the laser pulse and the photon detection is then measured for several million times. Detectors used for this purpose are extremely sensitive and are single photon sensitive detectors. They are photomultiplier tube, micro channel plate, a single photon avalanche diode or hybrid PMT [128].

Since fluorescence emission is a statistical process each photon can arrive at different time points. As shown in Fig. 2.2. the second laser pulse may not have any photons arriving at the detector when we count at the single photon level. Thus according to their 


\section{\begin{tabular}{l|l} 
Chapter 2 & METHODS
\end{tabular}}

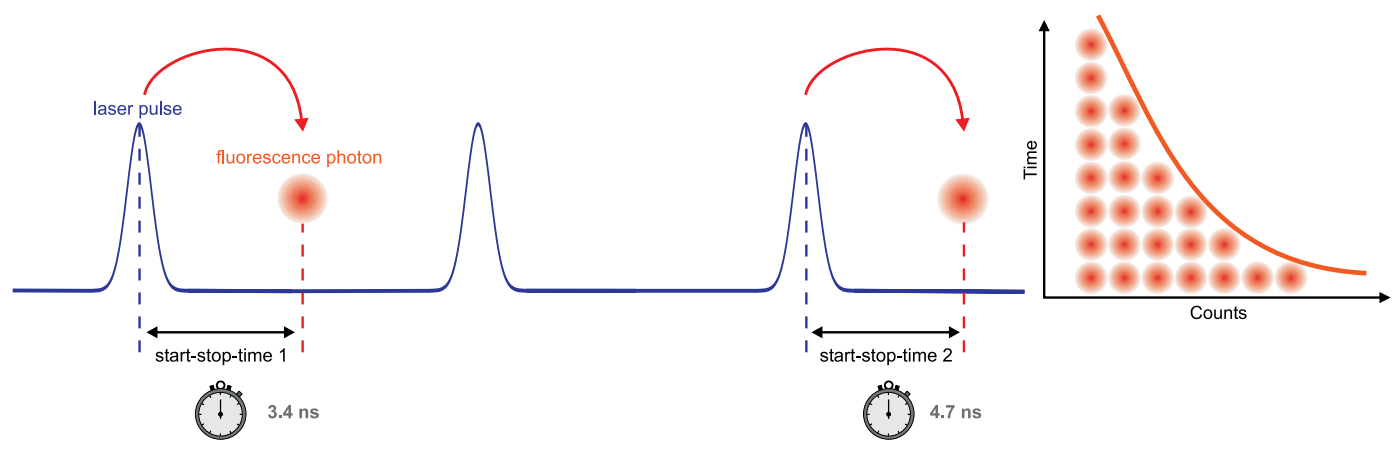

Figure 2.2.: Time correlated single photon counting. From the left: An electronics stopwatch based counting of photons. The start signal is assigned to the pulsing of the laser and the stop signal is counted when the photon arrives at the detector. There may be no photon as in the second laser pulse, or a different photon arrival time like in the third pulse. Such random photon arrivals are sorted out into a time binned histogram (extreme right) which plots the photon emission intensity (counts) over time, where the width of the time bin correlates to the stopwatch resolution [128].

arrival time they are sorted out into a histogram that consists of a range of time bins where each width of the time bin corresponds to stopwatch resolution. The typical profile of such a histogram is then an exponential decay of photons (given as intensity) over time (ns). In order to finally acquire a fluorescence lifetime measurement or image, the photons are assigned to different pixels. This is done by storing the absolute arrival times of photon plus the relative arrival time of the photon with respect to a laser pulse. For a more detailed understanding, one can refer to literature found in Wahl and Müller [129].

\subsection{Macrorheology}

Rheology deals with the branch of studying deformation of materials and their responses under the application of force. It is achieved by applying either a flow velocity (shear rate) in the case of liquids or by applying a force (stress) to a solid.

Ideal solids are described by Hooke's law and ideal liquids are given by Newton's law.

Real and biological materials are neither ideal solids nor ideal liquids, but have characteristics intermediate to both, displaying solid and liquid like properties at a given time giving rise to visco-elasticity. These materials are anisotropic in deformation i.e. the viscoelastic properties vary when deformations are applied in different directions.

An ideal elastic solid with a height $\mathrm{H}$ and an area $\mathrm{A}$ is subjected to a shear deformation of force $\mathrm{F}$ on the surface of its plane (Fig. 2.3. It leads to a deformation of the solid that results in an extension of its length $\Delta x$. The volume however remains constant. The stress 
a)

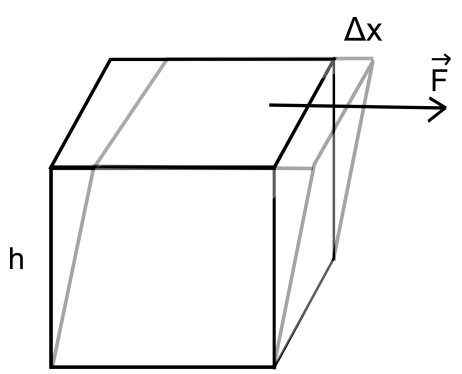

b)

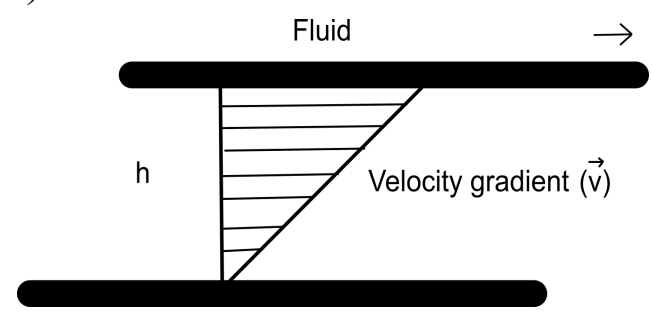

Figure 2.3.: Shear deformation of an elastic solid and viscous fluid. (a)The elastic solid is subjected to a shear force $\vec{F}$ for a given area, $\mathrm{A}$ and height, $\mathrm{h}$ which results in a deformation $\Delta x$. The stress and the strain is given by $\sigma=F / A$ and $\gamma=\Delta x / h$. (b) Similarly, a liquid when sheared flows with a varying velocity $\vec{v}$ across the different fluid layers for a given height ' $h$ '. The strain rate is then given as $\dot{\gamma}=\vec{v} / \mathrm{h}$.

acting on the solid is then given as the force acting per unit area $(\sigma=\mathrm{F} / \mathrm{A})$. The resulting deformation (strain) is the change in length of a material to its original length $(\gamma=\Delta x / h)$. It is a dimensionless quantity. The stress and the strain are related by the elastic shear modulus $(G)$ which gives the rigidity or stiffness of the system which is

$$
G=\frac{\sigma}{\gamma}
$$

In a viscous material, the stress is not proportional to the strain but rather to the rate of strain $(\mathrm{d} \gamma / \mathrm{d} t)$ with the unit $\sec ^{-1}$. When two plates containing a fluid are sheared, the fluid layers begin to move with different velocities (Fig. 2.3). The top layer moves at higher velocity than the bottom layer where fluid flow is almost stationary. Hence when strain is increased in a continuous manner, the change in the strain rate becomes more dominant than the strain itself which determines the fluid drag forces. The strain rate is given as $\dot{\gamma}=\vec{v} / \mathrm{h}$, where $\mathrm{v}$ is the velocity of the fluid $(\mathrm{m} / \mathrm{s})$ and $\mathrm{h}$ is the height or gap between two plates $(\mathrm{m})$. The strain rate $(\dot{\gamma})$ and stress $(\sigma)$ (i.e. force exerted on the fluid for a given unit area) are related through the proportionality constant shear viscosity $(\eta)$ as

$$
\eta=\frac{\sigma}{\dot{\gamma}}
$$

Viscosity describes fluids material property as a measure of the resistance to the flow [87, [54],[76],[1]. Stress and strain describes material properties of the system irrespective of their size and shape. In a rheometer, the stress is measured as a shear force applied via the torque and the strain is measured as the velocity of the rotational speed of the moving top plate. 


\section{\begin{tabular}{l|l} 
Chapter 2 & METHODS
\end{tabular}}

\section{Measuring Geometries}

Measuring geometries are discussed to show how rheology is affected by the geometry of plates used. In this thesis all measurements were made with the commercially available Anton-Paar Physica MCR 501 (Anton Paar GmbH, Ostfildern, Germany) in a cone plate geometry. It consists of a moving top plate which is available in different geometries and a stationary bottom plate. The cone plate and the parallel plate geometry (Fig. 2.4 will be briefly discussed with which time dependent measurements can be made more readily.

\section{Parallel Plate}

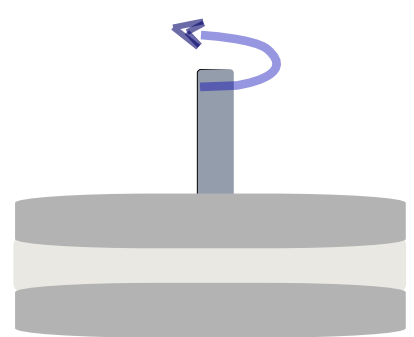

Cone Plate

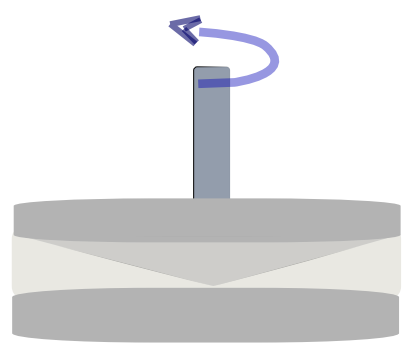

Figure 2.4.: Measuring geometries of a rheometer. The two widely used geometries in rheological measurements, cone plate (CP) and parallel plate (PP). The sample is filled between two plates. Arrowhead represents the rotation of the top plate which is oscillated at a particular strain, which leads to a deformation of the material. The gap distance between the plates and the diameters of the plates are not drawn to scale in this sketch.

The cone plate is one of the most widely used measuring geometry due to uniform shearing, possibility of measuring normal stresses and the feasibility of using low material volumes which makes it easier for biological samples that are expensive and available only in small amounts. The parallel plate geometry is preferred only where uniform gap conditions are a requisite, i.e. for materials where fluid flow shows a gap dependent behaviour or when fluid slips at the wall. Shearing is not uniform in this geometry type and large sample volumes are needed as the gap distance between two plates are larger than in CP. Normal stresses are also measured with parallel plate [61].

\subsubsection{Viscoelastic Measurements}

Viscoelastic measurements are carried out in biological materials over large time and deformation scales. It is described by $\mathrm{G}(\mathrm{t})$, a time-dependent modulus. Small amplitude oscillatory deformations are applied at a specific frequency in given intervals of time such that the material is disturbed only slightly from its equilibrium and a linear response can be measured. Such deformations in a cone and plate geometry are imposed by rotating the cone about its axis at an angular velocity that oscillates sinusoidally. 


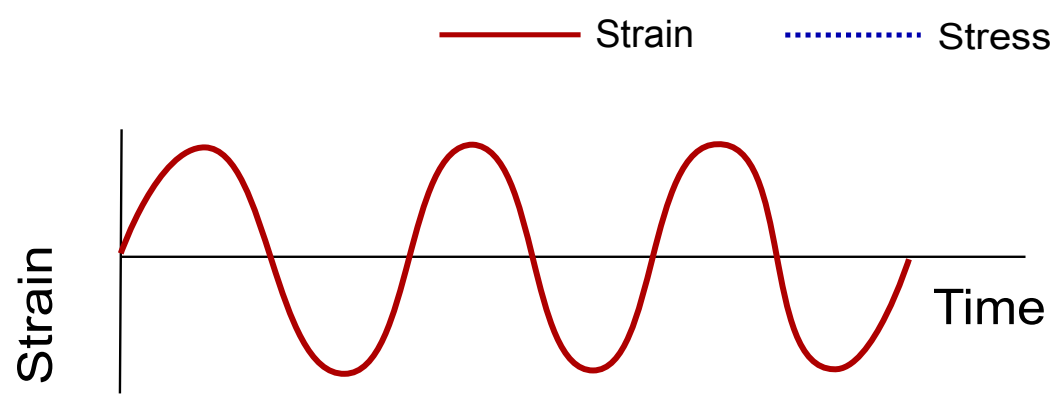

Elastic solid (stress and strain in phase)
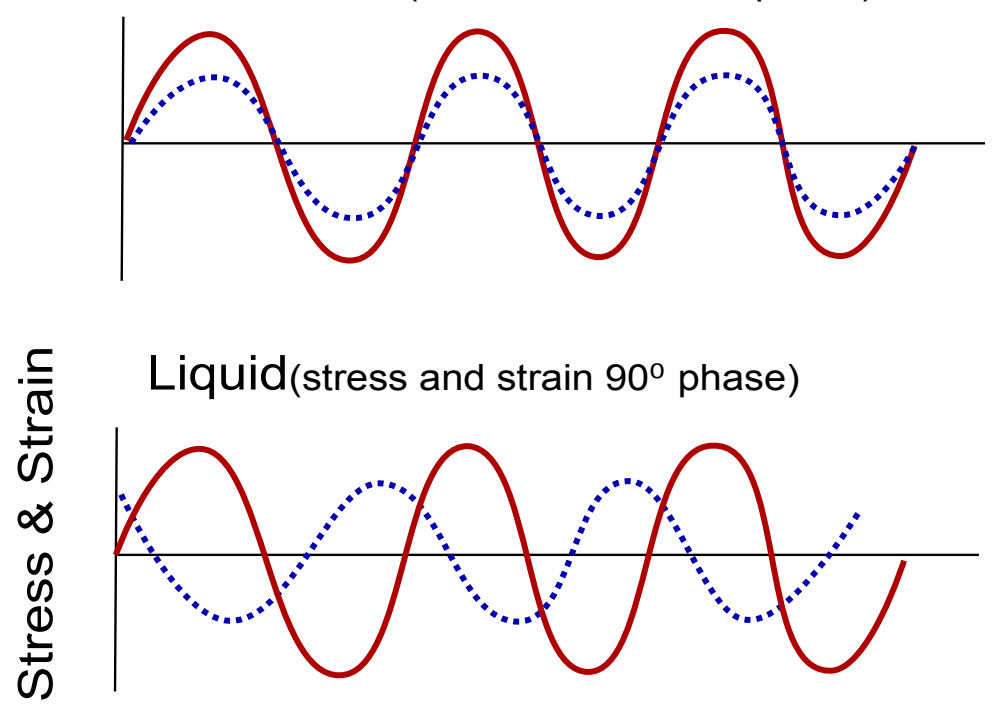

Viscoelastic (phase difference between $0^{\circ} \& 90^{\circ}$ )

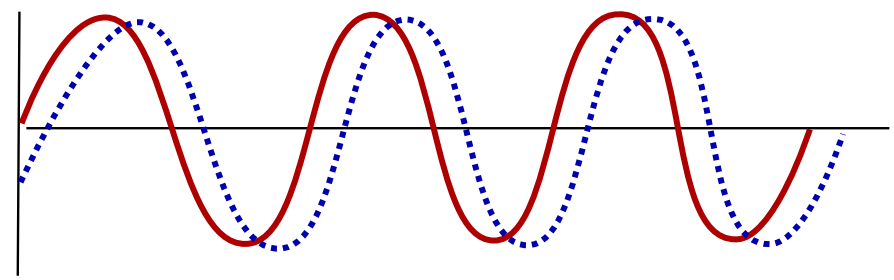

Figure 2.5.: A dynamic experiment illustrating responses of a solid, liquid and viscoelastic material. A strain with constant amplitude is applied to the material. The response of the material when its a solid, liquid and viscoelastic is shown beneath it. For a solid, the stress and strain are in phase, in a liquid it is phase shifted by $90^{\circ}$ and for a viscoelastic material, it has both the contributions in which the phase shift is between $0^{\circ}$ and $90^{\circ}$. The stress is represented by the dotted curve (blue) and the strain is given as a smooth curve (red). Figure has been redrawn after [87]. 


\section{\begin{tabular}{l|l} 
Chapter 2 & METHODS
\end{tabular}}

When a small oscillatory strain is applied to the material at a particular frequency $\omega$ the stress will oscillate with time, $t$ but will be phase shifted by $\delta$ with respect to strain as

$$
\begin{gathered}
\gamma(t)=\gamma_{0} \sin \omega t \\
\sigma(t)=\sigma_{0} \sin (\omega t+\delta)
\end{gathered}
$$

$\sigma=$ stress, $\gamma=$ strain, $\sigma_{0}=$ amplitude of stress, $\gamma_{0}=$ amplitude of strain, $\omega=2 \pi v=$ angular frequency $(\mathrm{rad} / \mathrm{s})$, where $v=$ frequency $(\mathrm{Hz})$ and $\delta=$ phase shift which is between $0^{\circ}$ and $90^{\circ}$ [54].

If a material is a pure elastic solid, then the stress and strain are in phase with each other $(\delta=0)$ (Fig. 2.5). For a purely viscous fluid, the stress and the strain are out of phase with each other $\left(\delta=90^{\circ}\right)$ (Fig. 2.5). For visco-elastic materials, $\delta=0^{\circ}$ to $90^{\circ}$ (Fig. 2.5). The stress then is a sum of both elastic and viscous contributions. The stress and strain for such viscoelastic materials is related as

$$
\sigma(t)=\gamma_{0}\left(G^{\prime} \sin \omega t+G^{\prime \prime} \cos \omega t\right)
$$

where $G^{\prime}$ is the elastic storage modulus, $G^{\prime \prime}$ is the viscous loss modulus.

In the above equation $\mathrm{G}^{\prime}$, the storage elastic component gives the ratio of stress component in phase with strain. $G^{\prime \prime}$ viscous loss modulus represents the ratio of stress component out of phase $90^{\circ}$ with strain. In an ideal elastic system $G^{\prime}=G$ and $G^{\prime \prime}=0$. In case of an ideal liquid (Newtonian) $G^{\prime \prime}=\omega \eta$ and $G^{\prime}=0$.

A linear viscoelastic regime, in a dynamic experiment, is when the ratio of stress and strain at a given frequency is not dependent on the magnitude of the strain. This holds valid for all materials when probed at small strains. When strain or strain amplitudes become larger, the stress and strain will not be proportional. The material will exhibit a strain dependent behavior and is said to be in the non-linear viscoelastic regime. They will either exhibit strain dependent stiffening or softening. 


\subsection{Microrheology}

Complex fluids exhibit rich linear viscoelastic behavior due to their inherent large length scales (ranging from $\mathrm{nm}$ to $\mu \mathrm{m}$ ) that arise from their structure. This leads to a complex time-dependent behavior. Therefore, to examine their mechanical properties, a wide frequency range is required. Although it can be determined from rheometer as $\mathrm{G}^{*}$ (complex shear modulus), the frequency range is limited to the inertia of the instrument which is $50 \mathrm{~Hz}$ [61].

Microrheology offers the advantage of probing viscoelastic materials in a wide frequency range from $0.1 \mathrm{~Hz}$ to $100 \mathrm{kHz}$ [11]. Also only small sample volumes are needed which is suitable for biological materials. In addition, the local rheological properties in inhomogenous materials can be studied [63]. This technique has vastly expanded due to significant advances in methods of force generation, detection and manipulation.

\subsubsection{Single Particle Passive Microrheology (1PMR)}

1PMR was used in this work to measure the local viscoelastic properties of actin and actinDNA sensor network. In 1PMR, tracers (beads) are embedded in the material and their displacements due to thermal fluctuations from Brownian motion are recorded.

A spherical bead thus embedded in an incompressible viscous fluid experiences a drag force $\vec{F}$ for a velocity $\vec{v}$ which is given by the Navier stokes equation for small Reynolds number as

$$
\vec{F}=\gamma \vec{v}=6 \pi \eta r \vec{v}
$$

$\gamma=$ drag coefficient, $\mathrm{r}=$ bead's radius, and $\eta=$ dynamic viscosity. The displacement of the particle is described by the Langevin equation of motion which gives the motion for an overdamped particle driven by a random force. This describes the motion of the bead in a liquid at a harmonic potential generated by the trap.

$$
m \ddot{x}(t)+\eta \dot{x}(t)+\kappa x(t)-f(t)-\zeta(t)=0
$$

where $\mathrm{m}=$ mass of the particle (bead), $\mathrm{f}=$ an external force, $\zeta=$ thermal force. Ensembling the average $\zeta(t)=0$ and applying Fourier transform one gets the response function $(\chi)$

$$
x(\omega)=\frac{1}{\kappa-m \omega^{2}-i \gamma \omega} f(\omega)=\chi(\omega) f(\omega)
$$

with $\chi=1 /\left(\kappa-m \omega^{2}-i \gamma \omega\right)$. The fluctuation-dissipation theorem (FDT) is applied to the response function $\chi$ to obtain the complex shear modulus $\left(\mathrm{G}^{*}\right)$. 


\section{\begin{tabular}{l|l} 
Chapter 2 & METHODS
\end{tabular}}

The convolution of Eqn. 2.11 in time domain can be written as

$$
x(t)=\int \chi\left(t-t^{\prime}\right) f\left(t^{\prime}\right) d t^{\prime}
$$

This convolution is an asymmetric function. $\chi(t)$ can be decomposed into an even and odd part. Before applying the FDT, a subtelty $t>0$ where the response function is non zero has to be considered.

$$
\chi(t)=\chi_{E}(t)+\chi_{O}(t)
$$

$\chi_{E}(t)=\frac{1}{2}(\chi(t)+\chi(-t))$ and $\chi_{O}(t)=\frac{1}{2}(\chi(t)-\chi(-t))$. The even part of the Fourier transform $\chi^{\prime}(\omega)$ is real and symmetric, the odd part referred to as $\mathrm{i} \chi^{\prime}(\omega)$ is imaginary and odd in $\omega$.

$$
\chi(\omega)=\chi^{\prime}(\omega)+i \chi^{\prime \prime}(\omega)
$$

Applying the FDT one gets,

$$
\chi(\omega)=\frac{i \omega}{k_{B} T}<x(\omega) x(-\omega)>
$$

The imaginary part in the above equation for all $t$ is given as

$$
\chi^{\prime \prime}(\omega)=\frac{\omega}{2 k_{B} T}<x(\omega) x(\omega)>=\frac{\omega}{2 k_{B} T}<\left|x(\omega)^{2}\right|>
$$

This is another form of expressing the fluctuation-dissipation theorem which has the term for power-spectral density $C(\omega)=<\mid x\left(\omega^{2} \mid>\right.$ for equilibrium fluctuations. The real part of the response function is obtained from the Kramers-Kronig relation.

$$
\chi^{\prime}(\omega)=\frac{1}{\pi} P \int_{-\infty}^{\infty} \frac{\chi^{\prime \prime}\left(\omega^{\prime}\right)}{\omega^{\prime}-\omega} d \omega^{\prime}
$$

$\mathrm{P}$ is the Cauchy principal value. Thus the power-spectral density can be used to get the response function of the particle $\chi(\omega)$. The generalised form of Stoke's law of Eqn. 2.9 given below can now be used to obtain the complex shear modulus of the material.

$$
\chi(\omega)=\frac{1}{6 \pi r G} F(\omega)=\chi(\omega) F(\omega)
$$

The stress and the strain are related for a linear response of the medium as

$$
\sigma(\omega)=G^{*} \epsilon(\omega)
$$

where $\sigma$ is a small oscillating stress, $\epsilon$ is the shear strain, $G^{*}$ is the complex shear modulus. It has a real part the storage modulus $\left(\mathrm{G}^{\prime}(\omega)\right)$ and an imaginary part, the loss modulus $\left(\mathrm{G}^{\prime \prime}(\omega)\right)$ [77],[32]. 


\subsubsection{Principle of Optical Trapping}

Arthur Ashkin in 1970 used the radiation pressure of light to trap small dielectric particles or objects (of microscopic dimensions) in a focused laser beam [9]. Since then small dielectric objects are trapped in the same manner with a high numerical aperture (NA) lens. Hence the term optical trap or more precisely optical tweezers, as the object is optically tweezed or trapped. Subsequently it has found diverse applications in fields of mechanoenzymes and biopolymers mechanics [126], [7], [81], [25], complex fluids microrheology [88], [102], [122] and molecular kinetic free energy measurements [6].

The forces measured or exerted are in the order of piconewton to tens of piconewton. The displacements of beads is measured with quadrant photodiodes at subnanometres spatial resolution. Detection is achieved with a back focal plane interferometry [94]. Near infrared lasers are generally used for trapping as they cause minimal radiation damage to biological materials [119].

The principle of optical trapping is that a force balance is achieved between refracted and scattered rays of an incident radiation depending upon the object's geometry and refractive index. In other words, the momentum of photons entering and leaving an object of microdimensions translates to a difference in the object's momentum. Hence one needs to consider the object's dimension (particle size) with respect to the wavelength of trapping light for momentum calculation which gives rise to two regimes.

Ray optics regime is used when dimension of the object (d) is much larger than the wavelength of trapping light $(\mathrm{d} \gg \lambda)$. Rayleigh regime is considered for $\mathrm{d} \ll \lambda$. Since in biophysics, the bead size $(\mu \mathrm{m})$ and wavelength of trapping light (visible or near infra red) are on the same order of magnitude, force calculations here needs to have an approach that is between the two regimes [58], [77], [14],[107].

\subsubsection{Detection via Back Focal Plane Interferometry}

In order to have a quantitative output of the force and displacement from an optical trap, position detection of the particle is recorded to have a high spatial and temporal resolution. Experimentally it is done as follows. The motion of the trapped particle in the focal volume of the trap is detected by interferomtery via the back focal plane detection. The trapping light after it passes through the sample gets scattered by it. The scattered and the trapping light (transmitted beam) that exits the sample are then collimated by a condenser. Superposition of these two beams results in an interferometry pattern at the back focal plane (BFP) of the condenser.

The characteristics of this pattern (relative to two beam positions) on the back focal 


\section{\begin{tabular}{l|l} 
Chapter 2 & METHODS
\end{tabular}}

plane is imaged onto split photodiodes called quadrant photodiode (QPD). This has four equal light sensitive segmented parts that detects intensity changes. This is translated into spatial intensity distribution. At the BFP, the intensity distribution of the beams do not change when the optical trap is moved around in the sample. It is only affected by the motion of the trapped bead with respect to the trap which causes a shift in the interference pattern on the BFP. This is why the back focal plane interferometry is used as a detection method as subnanometer displacements from the trapped bead are recorded that leads to a high spatial resolution. Scheme Fig. 2.6 shows the detection at the back focal plane of the condenser.



Figure 2.6.: Detection via back focal plane interferometry. The lateral displacement of the bead at the center of the trap is shown on the left side of the image. The intensity of the scattered and transmitted beam interferes at the back focal plane of the condenser which is imaged onto a quadrant photodiode. The distribution of intensities in the four quadrants is illustrated on the extreme right. Figure is taken from [58].

The Intensity distributions (differential signals) $D_{x}, D_{y}$ can be calculated as

$$
\begin{gathered}
\left\{\begin{array} { c } 
{ I _ { y + } = I _ { 1 } + I _ { 2 } } \\
{ I _ { y - } = I _ { 3 } + I _ { 4 } }
\end{array} \quad \left\{\begin{array}{l}
I_{x+}=I_{2}+I_{4} \\
I_{x-}=I_{1}+I_{3}
\end{array}\right.\right. \\
D_{y}=\frac{I_{y+}-I_{y-}}{I_{y+}+I_{y-}} .
\end{gathered}
$$

and

$$
D_{x}=\frac{I_{x+}-I_{x-}}{I_{x+}+I_{x-}} .
$$

For displacements around $\pm 250 \mathrm{~nm}$ the response of the QPD photocurrent to voltage remains linear without any crosstalk between the $\mathrm{x}$ and $\mathrm{y}$ channels. 


\subsubsection{Calibration of Trap Stiffness}
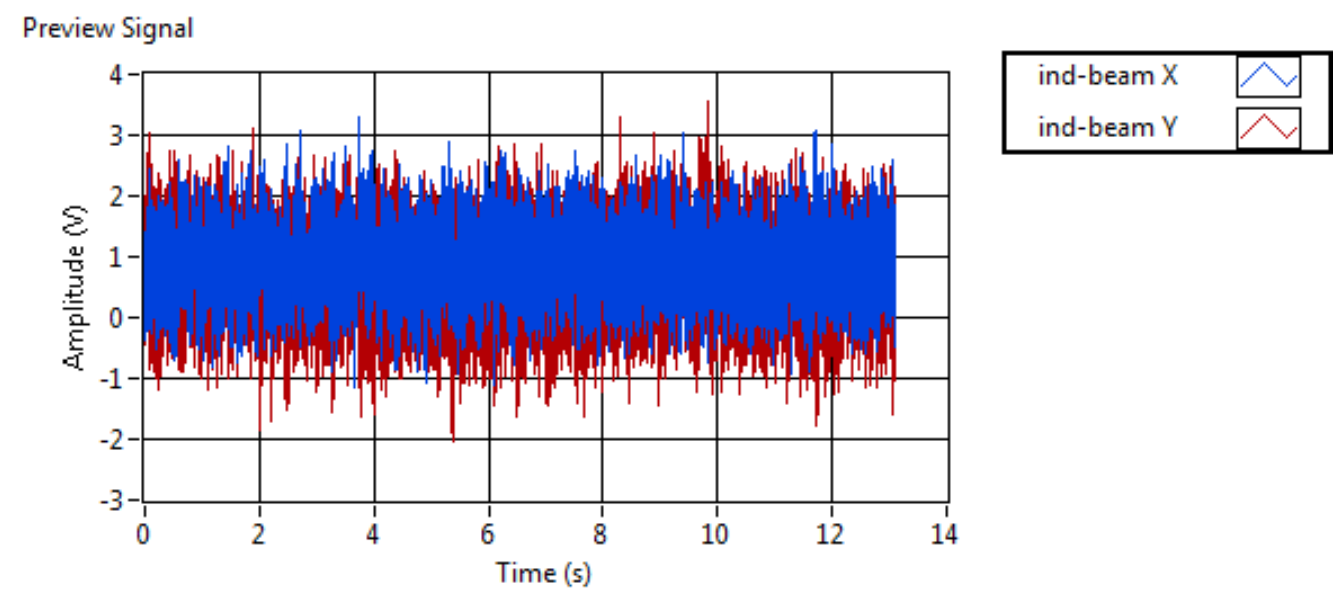

Preview Powerspectrum
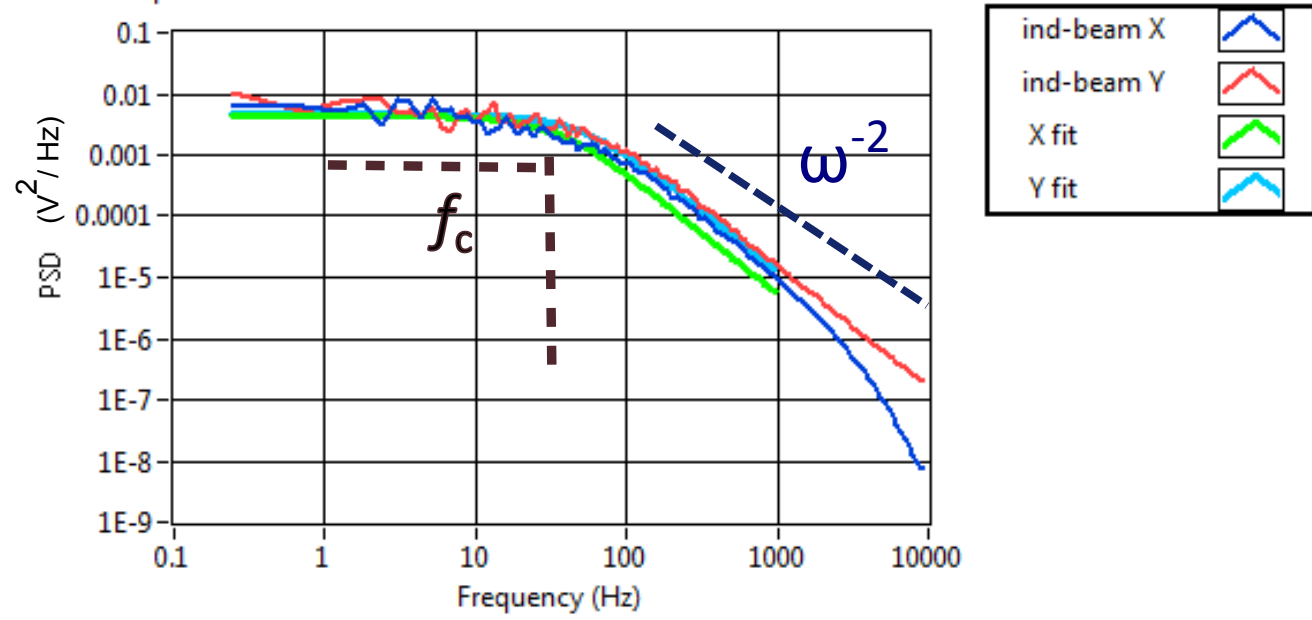

Figure 2.7.: Calibration of an optical trap using the power spectrum of an embedded particle. Upper image shows the displacement of a $1 \mu \mathrm{m}$ trapped particle (bead). The blue and red curves represent the bead's displacement amplitude in the $X$ and $Y$ direction. The lower image shows the bead's power spectral density (PSD) corresponding to its displacements. A Lorentzian fit of the PSD is shown in green and blue colors. $f_{c}$ is the corner frequency proportional to the trap stiffness. At low frequencies, a plateau regime is seen, whereas at higher frequencies the scaling of $\omega^{-2}$ represents free diffusion of the particle not confined to the trap. This figure is an exported preview interface of the LabVIEW trap commander analysis program.

The QPD's voltage signals have to be converted into physical units for measuring displacements and forces in the trap. This is done by calibrating the signal in the detector. The simplest approach is to employ the Brownian motion of a trapped particle in water and use the Langevin equation of motion which describes the motion of a dielectric 


\section{\begin{tabular}{l|l} 
Chapter 2 & METHODS
\end{tabular}}

particle in a viscous medium. This is given as

$$
m \frac{\partial^{2} x}{\partial^{2} t}+\gamma \frac{\partial x}{\partial t}+k x(t)+F(t)=0
$$

$\mathrm{m}$ - mass of particle, $\gamma$ - hydrodynamic drag coefficient, $k$ - trap stiffness, $\gamma \frac{\partial x}{\partial t}$ - drag force exerted on the particle from the medium and $k x$ - restoring force of the trap. $F(t)$ is the thermal force caused by Brownian motion and therefore has the time averaged to zero, $<F(t)>=0$. From the Langevin equation the theoretically expected form of PSD is a Lorentz function [44]. Hence the power spectrum of a particle's position can be calculated from the Lorentz function as [43] and Eqn 2.24

$$
S_{x}(f)=\frac{k_{B} T}{\pi^{2} \gamma\left(f_{c}^{2}+f^{2}\right)}
$$

where $k_{B}$ is Boltzmann's constant and $\mathrm{T}$ is absolute temperature and

$$
f_{c}=\frac{k}{2 \pi \gamma}
$$

is the characteristic frequency called 'corner frequency'. For frequencies $\left(f \ll f_{c}\right)$, the power spectrum is fairly constant indicating that the particle is confined within the trap. However, at higher frequencies $\left(f \gg f_{c}\right), S_{x}(f)$ it falls off with $1 / f^{2}$ (or $\omega^{-2}$ ), which represents the free diffusion of the particle. This means at shorter time scales the particle does not feel the confinement of the trap (see Fig. 2.7). To calculate the trap stiffness (k), the Stokes drag coefficient can be used. A more detailed description about power spectrum can be found in [43].

\subsubsection{Instrumentation of Optical Trap}

The instrumentation of the optical trap used in this thesis is discussed here. It is integrated into a custom built inverted microscope (Fig. 2.8). A $1064 \mathrm{~nm}$ laser which is used in this trap, is a near infrared solid state (Compass, Nd:YVO4, $1064 \mathrm{~nm}$, Coherent Inc., Santa Clara, CA, USA). It is linearly polarized by a $4 \mathrm{~W}$ laser and split into parallel and perpendicular beams to create two individual steerable traps at the specimen plane. Back reflections from the $1064 \mathrm{~nm}$ laser is prevented by using an optical isolator (IO-5-1064VHP,Thorlabs, Newton, NJ, USA).

The beam diameter is made upto 3 times larger with the help of a beam expander (Qioptic Photonics GmbH \& Co KG, Munich, Germany). It is approximately $4 \mathrm{~mm}$ in size in order to slightly overfill the objective. The intensity of the trapping power can be tuned with a motor-driven half-wave plate (PRM1Z8, Thorlabs, Newton, NJ, USA), alongside a polarizer. 


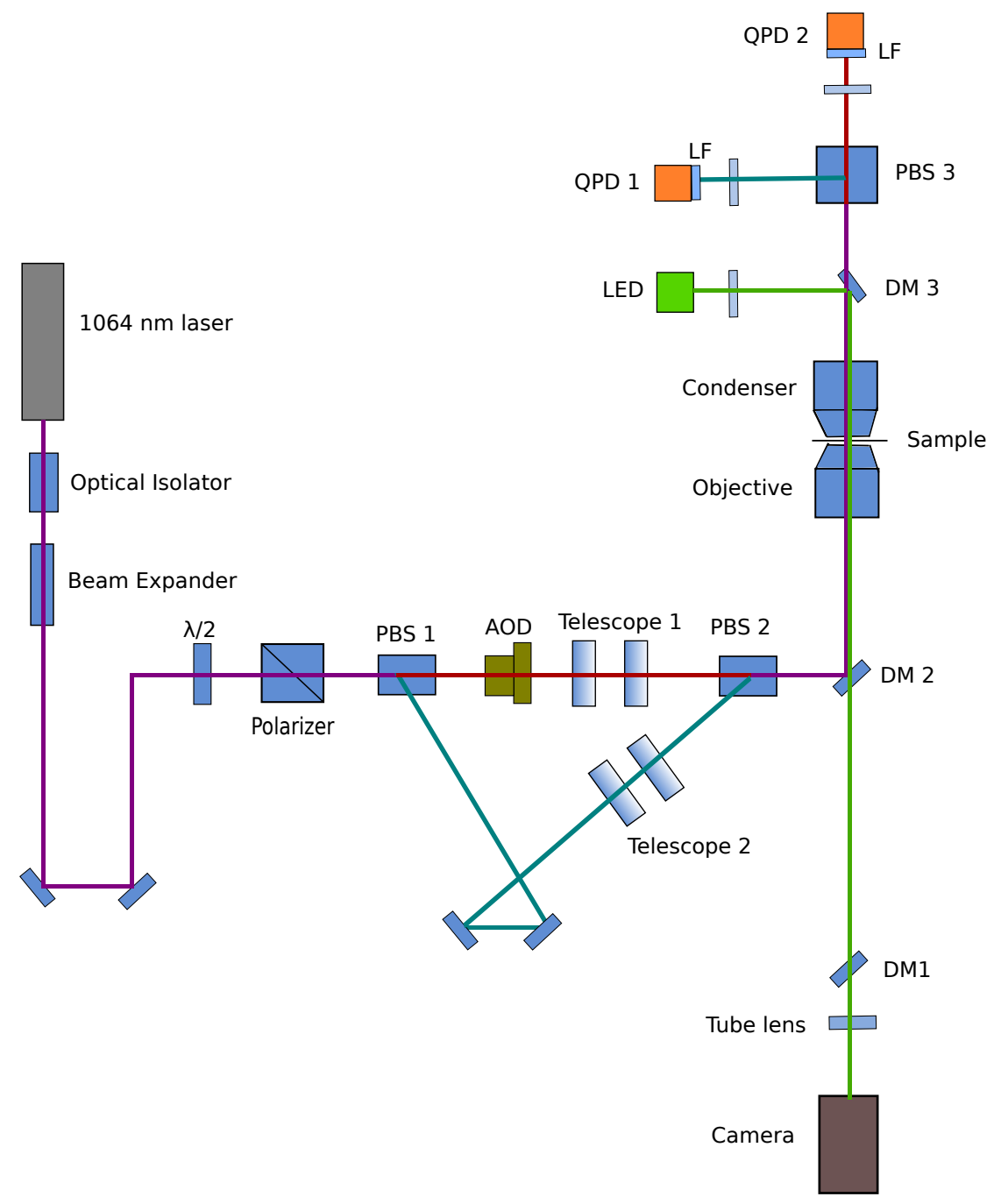

Figure 2.8.: A scheme of the optical trap set up. A $1064 \mathrm{~nm}$ laser passes through a polarizing beam splitter, gets split into two beam paths and is focused on the sample by the objective. The condenser collimates both the beams which are again split into two beams for their individual QPD detection (QPD 1 and QPD 2) via the back focal plane interferometry. Telescope lenses serve to steer these two beams, among which one of them can be steered fast by an AOD. A LED source illuminates the sample and is imaged by a camera. PBS - polarization beam splitter, DM-dichroic mirror, QPD - quadrant photodiode, LF - 1064 nm laser line filter.

The two orthogonally split beams pass through a 1:1 telescope lens (TL1 \& TL2) which helps to position the two traps individually at the specimen plane. A 2-axis acusto-optic deflector is introduced into the direct path before the telescope. Both of these beams are recombined again with a second glan laser polarizing beam splitter and is coupled into the objective with a dichroic mirror (DM2 in Fig. 2.8. A 100x oil immersion objective 


\section{\begin{tabular}{l|l} 
Chapter 2 & METHODS
\end{tabular}}

(NA=1.3, Neofluor, Carl Zeiss MicroImaging GmbH, Jena, Germany) focusses the laser beam into the sample.

A 3-D Translational stage which mounts the sample is steerable in the $x-y$ direction (with the help of micrometer screws) and in the z-direction with the help of a stepper motor. After the laser light passes through the sample it is collected by a 100x NA=1.4 oil immersion objective (Carl Zeiss MicroImaging GmbH, Jena, Germany). The light is split again into two orthogonally polarized beams by a third Glan laser polarizing beam splitter. The back focal plane of the condenser for each of the beam is focussed by a lens onto the quadrant photodiode (QPD) .

The laser beam before reaching the photodiode passes through a thin-film polarizer in order to reduce crosstalk and clean up the polarization signal and through a laser line filter (LF) which allows only $1064 \mathrm{~nm}$ trapping light to pass through. The currents in the QPD are converted to volts which measures the intensity distributions of the two lateral direction displacements [110]. In order to image the sample, a green LED is coupled into the microscope with a dichroic mirror which is present above the condenser. A second dichroic mirror couples the trapping laser light and the green LED into the microscopic path and is imaged onto a CCD camera (Coolsnap EZ, Photometrics, Tucson, AZ, USA). Since in this thesis single particle passive microrheology (1PMR) was employed, only the indirect beam path was used. 


\section{Force Sensor Characterization}

We have developed DNA based reversible molecular force sensor. This chapter focuses on testing and characterizing DNA sensors. The conformational states of sensors are reported through bulk fluorescence measurements in a spectrometer. The characterization of sensor is done via fluorescence lifetime imaging microscopy (FLIM) in DNA and actin buffers. The design of the sensor [80] will be recapitulated. Throughout this and the next chapter for convenience, the following abbreviations will be used. F - fluorophore strand, $\mathrm{H}$ hairpin, Q - quencher strand.

\subsection{Sensor Design}

The sensor consists of a hairpin which has a stem, a loop and two arms with which it can hybridize to the F and $\mathrm{Q}$ strand. The stem of the hairpin is 8 base pairs (bp) long, the self looping structure has $16 \mathrm{bp}$ and each of the arms (F strand with the fluorophore Alexa 488 and the $Q$ strand with quencher) have $20 \mathrm{bp}$. When the sensor is assembled (closed state), the fluorophore and quencher present on the inner ends of their respective strands (indicated by stars in Fig. 3.1) undergo FRET and contribute to a quenched (low) fluorescence. At a threshold force, the sensor opens. To characterize the sensor in the opened state, we employ a complementary strand, C. The sequence of this strand is complementary to the loop region of the hairpin. Thus, it hybridizes to the loop by competing with the stem's binding energy (Fig. 3.1. We characterize the quenched and open state of 


\section{\begin{tabular}{l|l} 
Chapter 3 & FORCE SENSOR CHARACTERIZATION
\end{tabular}}

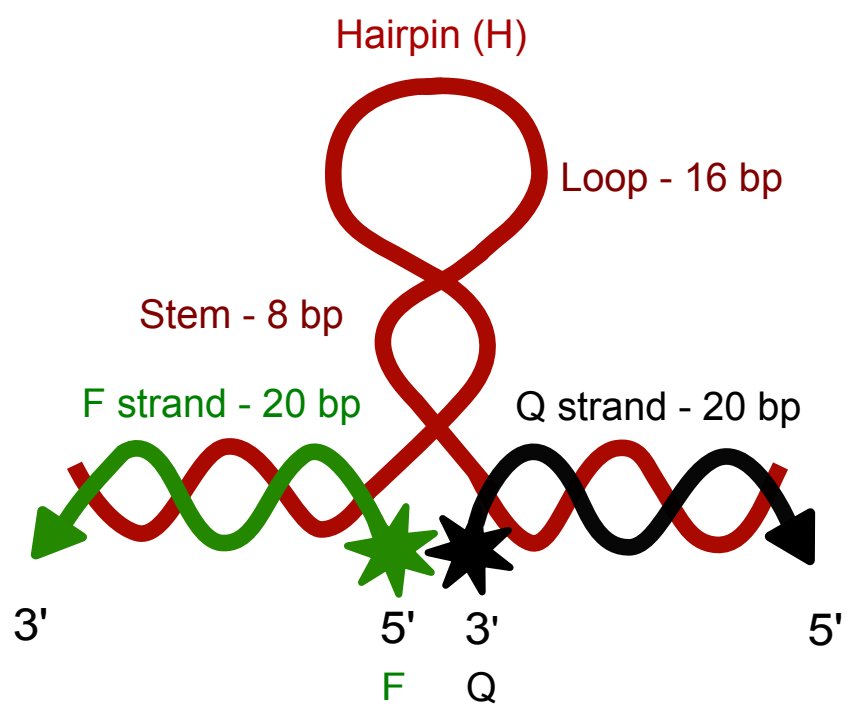

Figure 3.1.: DNA force sensor design. The force sensor consists of a hairpin with a $16 \mathrm{bp}$ loop and $8 \mathrm{bp}$ stem with two arms each of $20 \mathrm{bp}$ length. On one of its arms, it hybridizes with the F strand (20 bp) which has the fluorophore Alexa 488. The Q strand, also 20 bp in length, has the quencher and hybridizes with the hairpin on the other arm. $F$ and $Q$ form the FRET pair (present on the inner ends). F - Fluorophore, Q - Quencher, F strand - strand with a fluorophore, H - Hairpin, Q strand - quencher strand. Outer ends of the sensor (triangles) indicate the possibility of modification in $\mathrm{F}$ and $\mathrm{Q}$ strands to attach the sensor to the biological component of interest.

sensors via FLIM and thereafter estimate the FRET efficiency for our DNA sensors. This is a reversible sensor, meaning it can switch to the quenched position when forces cease.

The conformational state of DNA sensor is represented schematically for the quenched position in Fig. $3.2 \mathrm{a}$ and for the opened state in Fig. 3.2p. This sensor is a FRET based sensor where the process is strongly dependent on the distance between the two dyes [35]. Thus, in the closed conformation, the flurophore and quencher are brought near each other which leads to a high FRET (quenched fluorescence). The reason we use quencher as an acceptor in the FRET pair is that it gives a high signal/background ratio, where a high increase in fluorescence can be observed due to more efficient quenching [57]. As a part of the current thesis work, we also developed a control for the sensor termed as controls. We characterized its conformation through fluorescence in a spectrometer and lifetime from FLIM measurements. It has the same structure as the DNA sensor but lacks the quencher on the $Q$ strand. Its confirmation is represented schematically for the closed state in Fig. 3.2. and open state in Fig. 3.2 d. In the controls, FRET does not occur and therefore we expect the fluorescence and fluorophore's lifetime in FLIM measurements to remain unaltered for the closed and open states. 


\section{Force Sensor}

a)

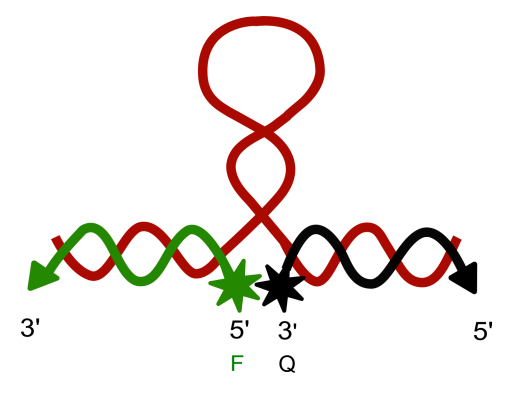

b)

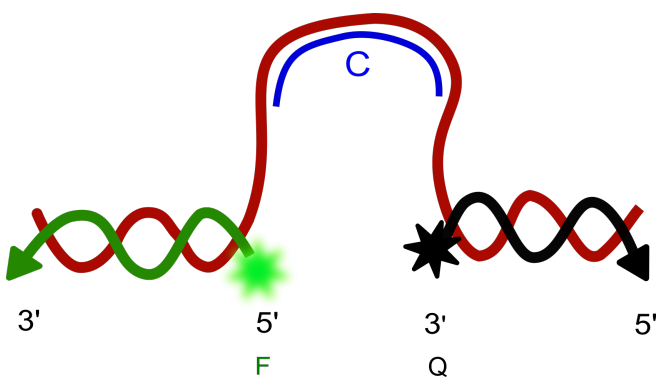

\section{Controls}

c)

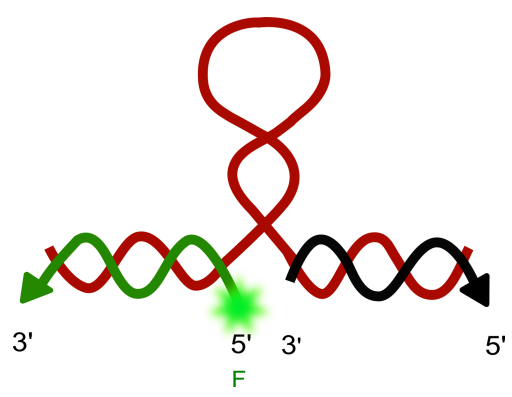

d)

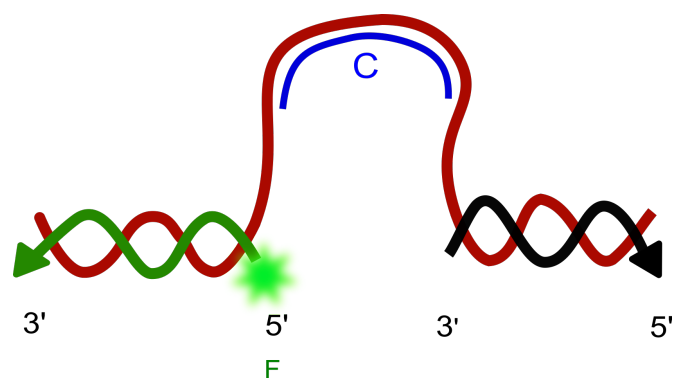

Figure 3.2.: DNA force sensor and controls fluorescence conformation. Schematic representation of a) quenched state of the DNA sensor (zero force) and b) open fluorescence state (threshold force opening). Controls have the same structural assembly but do not bear the quencher molecule on the $Q$ strand. c) closed state and d) open state of controls. The sensor is opened through C (complementary) strand shown in blue.

\subsection{Spectrometer: Bulk Fluorescence Intensity}

\subsubsection{Assembly and Conformation of Sensor via Bulk Fluorescence Measurements}

The assembly of sensors, its functionality in DNA buffer and when attached to actin was tested by measuring the bulk fluorescence intensity in a spectrometer. This was achieved by performing an emission spectrum to measure its fluorescence upon sequential addition of each strand of the sensor.

Figure 3.3 a shows the normalized intensity of sensors upon the sequential addition of each sensor component, indicating that it functions as hypothesized. All strands were used at the same molar concentration $(2 \mu \mathrm{M})$, except the complementary $(\mathrm{C})$ strand (20 


\section{\begin{tabular}{l|l} 
Chapter 3 & FORCE SENSOR CHARACTERIZATION
\end{tabular}}

$\mu \mathrm{M})$. After measuring the fluorescence of the fluorophore $(\mathrm{F})$ strand (green), the hairpin $(\mathrm{H})$ strand was then added (red) which leads to a slight reduction in fluorescence. The addition of the quencher $(\mathrm{Q})$ strand quenches the fluorophore due to FRET which is observed as strong decrease in fluorescence intensity (black). The sensor is now in its quenched state. By adding the complementary $(C)$ strand, the sensor is opened via the stem of the hairpin, spacing the FRET dyes apart and increasing the fluorescence. Figure 3.3 shows the time trace upon the sequential addition of sensors components. Although we have developed different stem lengths of the hairpin $(8,16,24 \mathrm{bp})$, we use the hairpin with 8 bp stem length in this thesis. For characterization purposes, we employ the complementary (C) strand designed for hairpin 8 , that binds to its loop region by overcoming the energy of the stem.

\section{Assembly of DNA Sensors}

a)

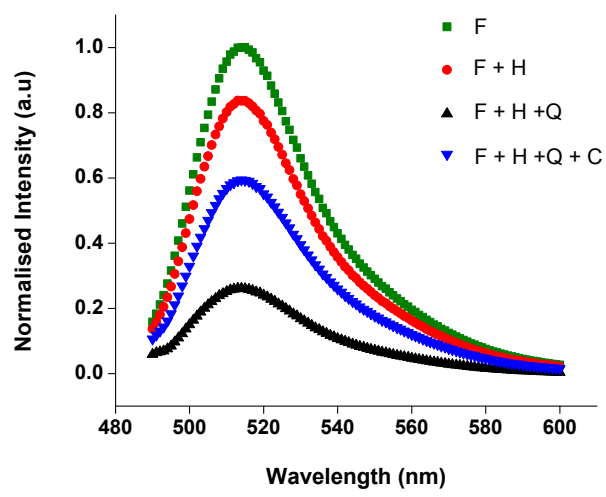

b)

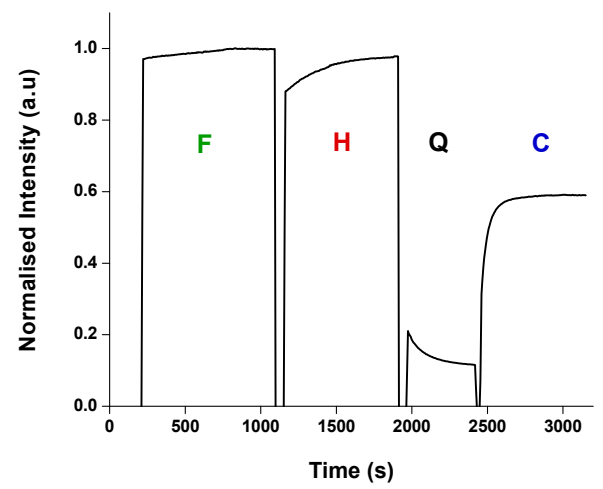

Figure 3.3.: Assembly of sensors in DNA buffer. Verification of DNA sensors proof of principle through a fluorescence emission scan. a) The sequential addition of $F, H, Q$ \& $C$ strands of the sensor is shown. After the addition of $Q$ strands, a quenched fluorescence occurs (black curve). The opening of the sensor with the $C$ strand is observed as an increase in the fluorescence (blue curve). b) Validation of the working of the sensor as a time trace experiment.

\subsubsection{Hairpin Opening: Binding Energy}

In this experiment we tested the opening of hairpins with different stem lengths (hairpin $16 \& 24)$ via the $C$ strand that is designed to open hairpin 8 . The experiment was performed in the same manner as before, where sensor components were sequentially added. The opening of the hairpin occurs via the rupture of hydrogen bonds in the stem. As shown in Fig. 3.4. the $\mathrm{C}$ strand is able to successfully open the hairpin structure with stem of $8 \mathrm{bp}$, but cannot compete with the binding energy of longer stems (hairpin 16 \& 24). From this observation, it is clear that opening of sensors is governed by the energy $(\Delta G)$ of the stem which is given by the number of base pairs on the stem and its GC con- 

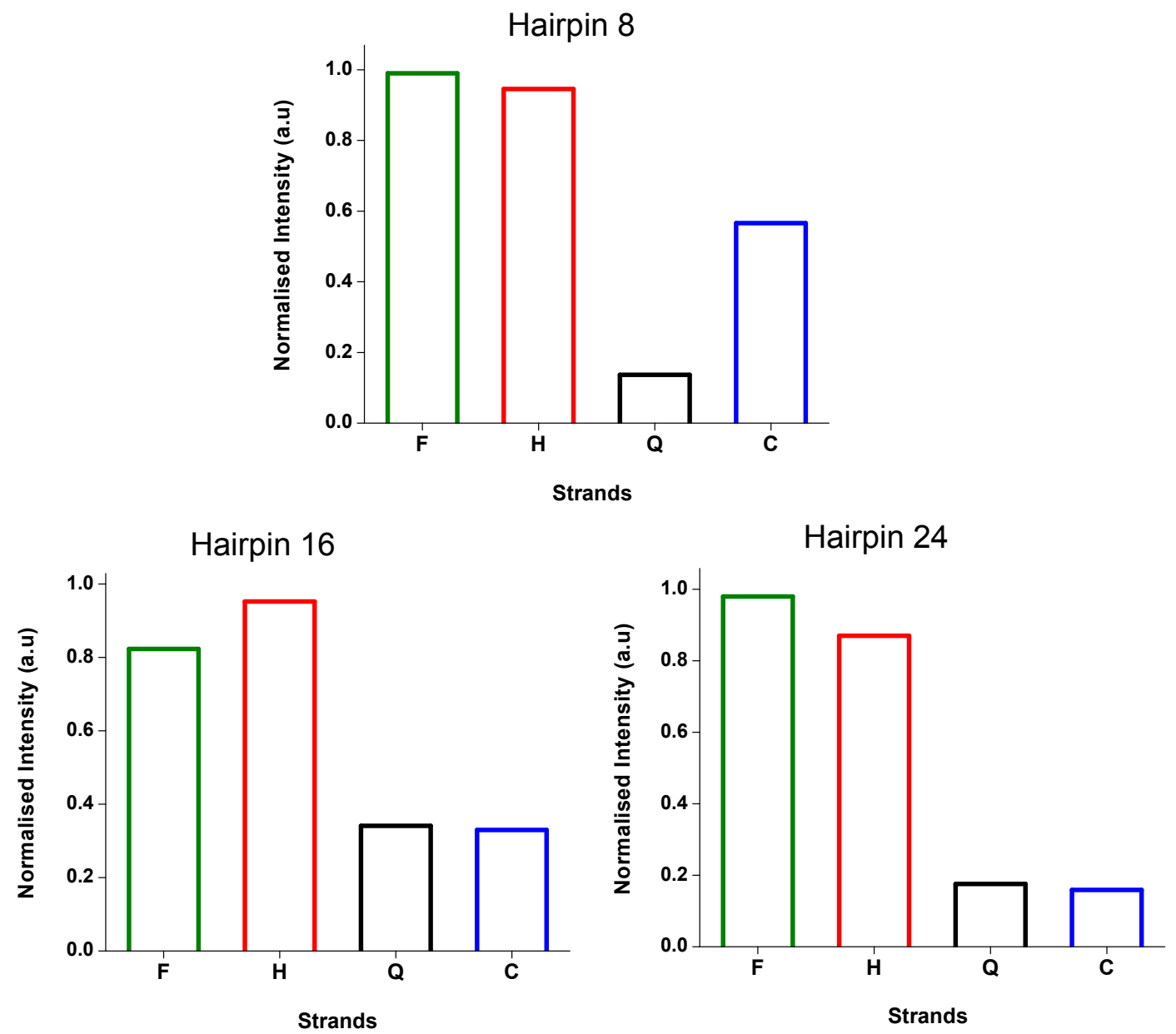

Figure 3.4.: Opening of hairpins governed by their stem energies. Top: The opening of sensors by the $C$ strand is seen from the increased fluorescence intensity (blue). The $C$ strand that is designed for hairpin 8 is tested in sensors with different hairpin stem lengths, the $16 \mathrm{bp}$ (bottom left), and 24 bp (bottom right). It does not unravel both of these hairpins as its energy is lower than the energies of these stems.

tent. C strand competes with the hairpin 8 stem's energy, overcomes it, thus causing the stem to separate [90]. In the case of hairpin 16 and hairpin 24, the energy of $C$ strand is insufficient compared to the energy of the stem region in these longer stems (hairpin 16 \& 24). Therefore even after the addition of $C$ strand, the quenched fluorescence can still be observed for hairpin 16 and 24 in Fig. 3.4 


\section{\begin{tabular}{l|l} 
Chapter 3 & FORCE SENSOR CHARACTERIZATION
\end{tabular}}

\subsubsection{Bulk Fluorescence: DNA Sensors versus Controls}

a)

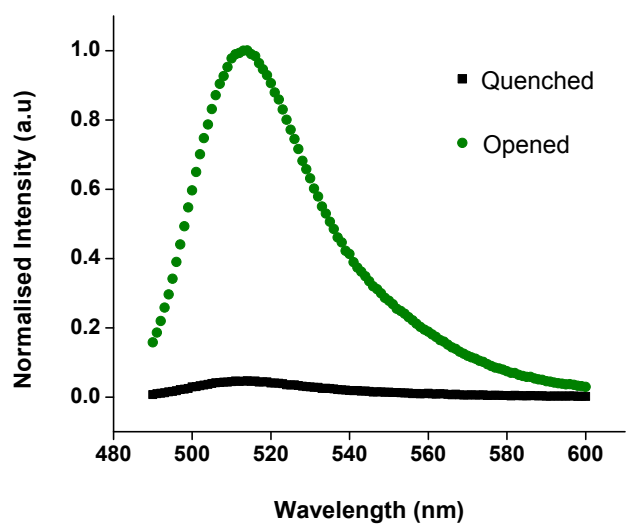

b)

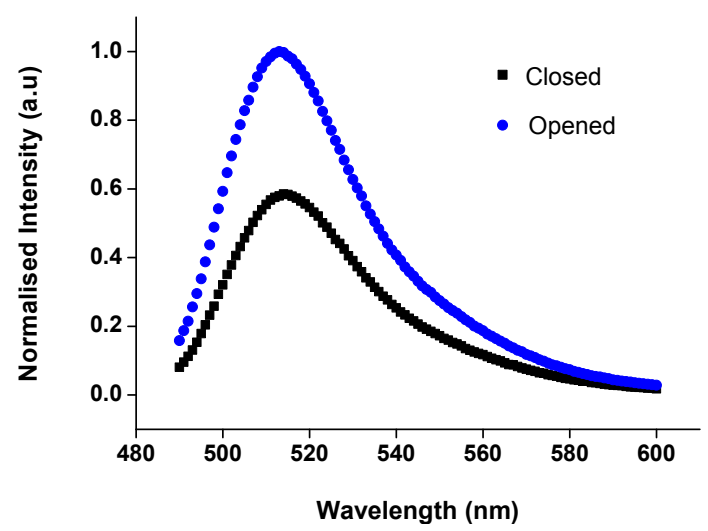

Figure 3.5.: Fluorescence of DNA force sensor versus controls in a spectrometer. a) Fluorescence of quenched sensor (black curve) and opened sensor (green curve). b)Closed controls (black curve) and opened controls (blue curve). The values plotted were averaged for $n=5$.

The controls that had been developed in this thesis do not bear the quencher. This is notated as $\mathrm{Q}^{-}$to show the lack of quencher in the $\mathrm{Q}$ strand. In this section, we quantify the bulk fluorescence of controls and sensors, to show the FRET-based proof of concept of DNA sensors which is not the case for controls. In Fig. 3.5, the left side shows a strongly quenched fluorescence (black curve) of closed sensors and an increased fluorescence from opened sensors (green curve). The fluorescence of controls in both the states, closed and open shows an unchanged bright fluorescence (Fig. 3.5p) indicating that no FRET occurs.

\subsubsection{Structural Quenching: Reduction in Fluorescence Intensities}

To observe the presence of structural quenching, we measured fluorescence intensities of individual $\mathrm{F}$ strands and $\mathrm{F}+\mathrm{H}$ strands (partially assembled sensor). We also measured the $\mathrm{F}+\mathrm{H}+\mathrm{C}$ strand's fluorescence, where the $\mathrm{F}+\mathrm{H}$ assembly is opened by the addition of the C strand. It is still a partially assembled sensor, where the loop is held open with the $C$ strand (Fig. B.2). From Fig. 3.6, the $\mathrm{F}+\mathrm{H}+\mathrm{C}$ strand (the blue curve) shows a clear peak in fluorescence intensity which is not observed in either individual $\mathrm{F}$ strands (green curve) nor $\mathrm{F}+\mathrm{H}$ (red curve). Although we expect a high fluorescence signal from these strands, their reduced fluorescence indicates the presence of structural quenching. This may arise due to the guanine bases present in the stem region of the $\mathrm{H}$ strand which may quench the Alexa 488 on the F strand which is elaborated under discussion (sec 3.3.8). From this experiment, we understood the contribution of each sensor strand towards the total fluorescence intensity. It also helped us gain insight into the interaction of the fluorophore 


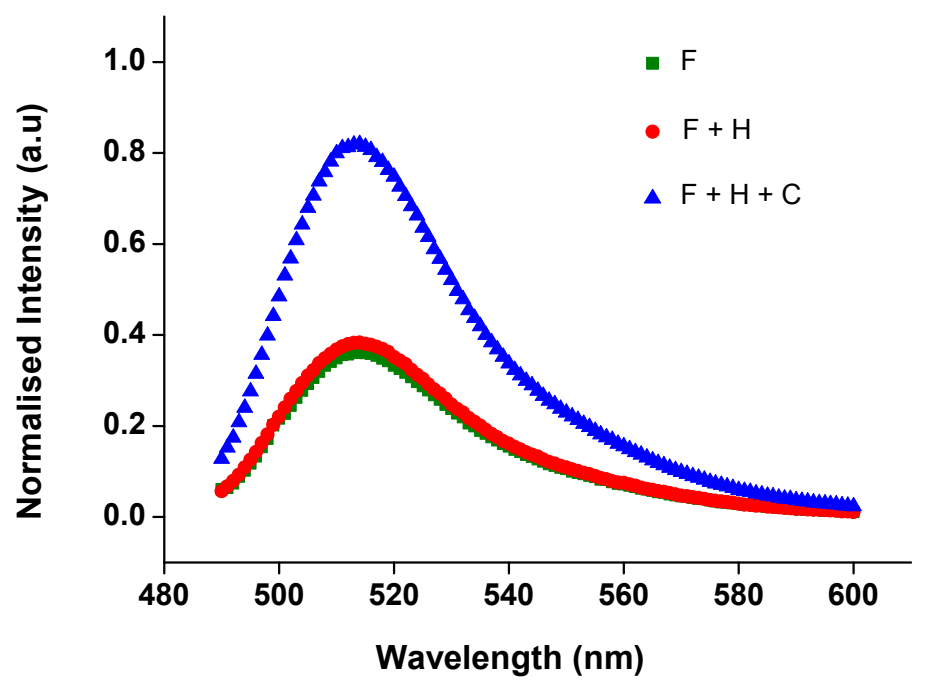

Figure 3.6.: Structural quenching from strands.. Strands F (green curve) and F\&H (red curve) show the presence of structural quenching which is observed as a reduced fluorescence. This quenching disappears in the $\mathrm{F}+\mathrm{H}+\mathrm{C}$ assembly which is seen as an increase in fluorescence (blue curve). Note: Red and green curves lie on top of one another.

Alexa 488 with F strand and strand assemblies $(\mathrm{F}+\mathrm{H}$ and $\mathrm{F}+\mathrm{H}+\mathrm{C})$ thereby indicating the presence of structural quenching.

\subsection{Sensor Characterization via Fluorescence Lifetime Imaging Mi- croscopy (FLIM)}

The advantage of lifetime measurements are discussed in Chapter 2 under FLIM (sec 2.2.3. When quenchers are used as one of the FRET pair, lifetime measurements (where the lifetime of the donor in FRET pair is measured in the presence and absence of acceptor) are commonly employed to characterize a FRET pair and estimate the FRET efficiency. It is also useful to determine if static quenching (contact quenching) accompanies FRET quenching. This can be investigated through fluorescence lifetime measurements. In static quenching, the fluorophore and quencher aggregate thus forming a physical association of a ground state complex [57]. When FRET quenching occurs the fluorescence intensity and lifetime is decreased. But in the static quenching mechanism, the fluorescence lifetime does not change as it occurs before the fluorophore absorbs a photon [57]. Since we have a quencher (Iowa black) in our FRET pair, we can also use FLIM to observe for any possible existence of static quenching in our DNA sensors. FLIM experiments were carried out in DNA hybridization buffer and actin buffer since they have varying amounts of salt compositions (Table A.1. A.2). This was done to validate the functionality of sensors in both of these buffers. 


\section{\begin{tabular}{l|l} 
Chapter 3 & FORCE SENSOR CHARACTERIZATION
\end{tabular}}

\subsubsection{Experimental Procedure}

Fluorescence lifetime measurements were done at the lab of Jörg Enderlein, University of Göttingen. This was performed using the commercial confocal setup Microtime 200 (PicoQuant GmbH, Berlin, Germany). The system is based on an Olympus IX-71 inverted microscope (Olympus Deutschland, Hamburg, Germany) amended with a side-port on the right side. The excitation unit consists of a pulsed diode laser $\left(\lambda_{\text {exc }}=485 \mathrm{~nm}\right)$ having a pulse width of 50 ps FWHM and repetition rate of $20 \mathrm{MHz}$. Additionally, a clean-up filter (BrightLine FF01-488/10, Semrock) was used in the excitation path. A polarisationmaintaining single-mode optical fibre (PMC-400-4.2-NA010-3-APC-250 V, Schäfter and Kirchhoff, Hamburg, Germany) was used to guide the laser light towards the microscope. The main optical unit consists of a fibre output that collimates the light into a beam of $12 \mathrm{~mm}$ diameter via an infinity-corrected $4 \mathrm{x}$ objective (UPISApo $4 \mathrm{X}$, Olympus). This beam was reflected by a quad-band dichroic mirror (Di01-R405/488/561/635, Semrock) towards the objective lens through the side port of the microscope. The laser power was maintained at $10 \mu \mathrm{W}$ at the back-focal plane of the objective lens. A high numerical aperture objective (UApoN 100X oil, 1.49 N.A., Olympus) was used to focus the light into the sample and was also used for collecting the fluorescence emission. Emission light was passed through the dichroic mirror and focused into a pinhole (diameter $100 \mu \mathrm{m}$ ) for confocal detection. After the pinhole, the light was refocused onto an avalanche photo diode (Excelitas technologies) using two achromatic lens doublets. In order to block backscattered excitation light, a long-pass filter was used. Additionally, we used a band-pass filter (Brightline HC5250/45, Semrock) before the detector. The dark count rate of the detector was less than 150 counts per second. Signals from the detector were processed by a multi-channel picosecond event timer (Hydraharp 400, PicoQuant) with 16 ps time resolution. Time-correlated single-photon counting histograms (TCSPC) were computed from the timed photon signals. For the solution measurements (DNA sensors in buffers), a droplet (20 to 30 microliter) of millimolar concentration of the sample was placed on a glass coverslip on the microscope stage. Recording of lifetime data was done at $30 \mu \mathrm{m}$ into the solution from the surface.

\subsubsection{FLIM: Structural Quenching of Sensors}

Fluorescence lifetime measurements were done on individual $\mathrm{F}$ strands, $\mathrm{F}+\mathrm{H}$ and $\mathrm{F}+\mathrm{H}+\mathrm{C}$ assemblies as separate measurements. The aim of this experiment was to measure the lifetime of fluorophore after the addition of each sensor strand. In this manner, structural quenching in DNA sensors, if present can be validated. Guanine bases in a DNA quench the fluorophore present next it due to a photoinduced electron transfer [112]. We refer to this as structural quenching.

Experimentally, a TCSPC histogram was computed from the recorded photons. For F, $\mathrm{FHQ}^{-}, \mathrm{FHQ}^{-} \mathrm{C}$ and FHQC strands, a mono-exponential decay function was fitted to the 
tail of the histogram ( $0.5 \mathrm{~ns}$ after the maximum) using a maximum likelihood procedure. The fitting function was

$$
I(t)=\frac{A}{\tau} e^{-(t / \tau)}+b
$$

where $\tau$ is the fluorescence lifetime, $\mathrm{A}$ is the amplitude, and $\mathrm{b}$ is the background. The negative $\log$-likelihood $\sum_{i} \mathrm{~h}\left(\mathrm{t}_{i}\right) \log \left(\mathrm{I}\left(\mathrm{t}_{i}\right)\right)-\mathrm{I}\left(\mathrm{t}_{i}\right)$, where $\mathrm{h}_{i}$ is the recorded TCSPC histogram and the sum runs over all TCSPC channels, was minimized using a Nelder-Mead simplex algorithm. From the fitting of the fluorescence decay curves in Fig. 3.7 we obtain the corresponding strands lifetimes. The trend of lifetime decay curves in Fig. 3.7 indicates that $\mathrm{F}$ (red curve) and $\mathrm{F}+\mathrm{H}$ (blue curve) have shorter decay curves with lifetimes of 3.51 $\pm 0.05 \mathrm{~ns}$ and $3.66 \pm 0.07 \mathrm{~ns}$ (Table 3.1). When $\mathrm{C}$ strand is added i.e $\mathrm{F}+\mathrm{H}+\mathrm{C}$, a completely longer decay (green curve) with a lifetime of $4.03 \pm 0.05 \mathrm{~ns}$ is observed. This is closer to the lifetime of a free Alexa 488 dye in solution (4.1 ns) [3]. Such a reduction in the lifetime of $\mathrm{F}$ and $\mathrm{F}+\mathrm{H}$ might be due to the presence of guanine bases in these strands lying close to the fluorophore.

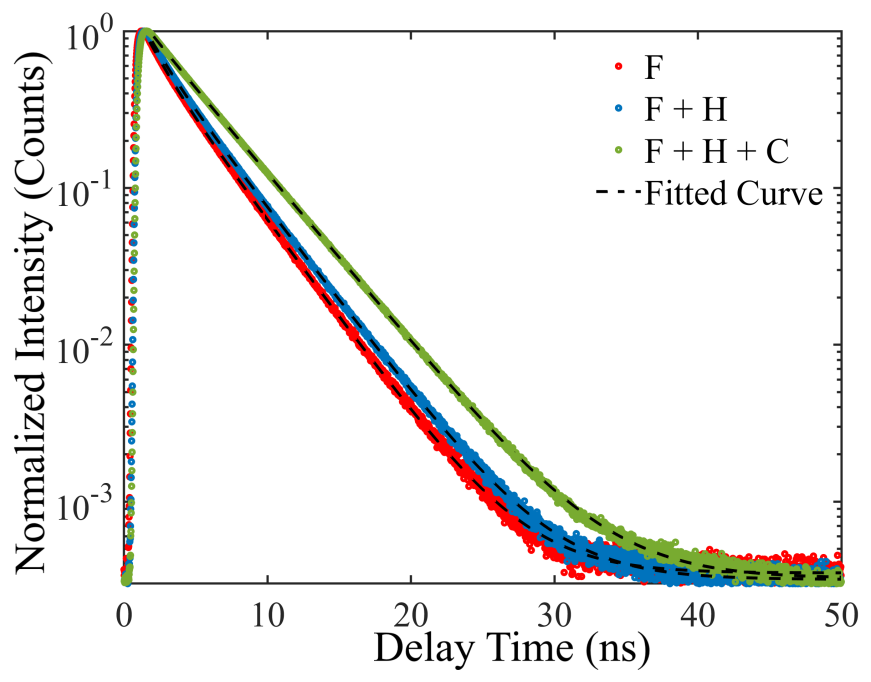

Figure 3.7.: Lifetime decay curves of different strands. Fluorescence lifetime decay curves of the $\mathrm{F}$ strand, $\mathrm{F}+\mathrm{H}$ strand, and $\mathrm{F}+\mathrm{H}+\mathrm{C}$ assembly are depicted. $\mathrm{F}$ and $\mathrm{F}+\mathrm{H}$ strands have a reduced lifetime (red and blue curves) which is seen below the longer lifetime curve of $\mathrm{F}+\mathrm{H}+\mathrm{C}$ (green). All strands are $2 \mu \mathrm{M}$ in concentration, except the $\mathrm{C}$ strand which is $20 \mu \mathrm{M}$.

The lifetime of free $\mathrm{F}$ strands is less than $\mathrm{F}+\mathrm{H}$ lifetime. This might be due to the reason, that, these $\mathrm{F}$ strands being thermally unstable may wrap around the fluorophore leading to a reduced lifetime than $\mathrm{F}+\mathrm{H}$ and $\mathrm{F}+\mathrm{H}+\mathrm{C}$. The lifetime of strands in decreasing order are $\mathrm{F}<\mathrm{F}+\mathrm{H}<\mathrm{F}+\mathrm{H}+\mathrm{C}(3.51 \pm 0.05<3.66 \pm 0.07 \mathrm{~ns}<4.03 \pm 0.05 \mathrm{~ns})$. In these measurements, we also find the presence of a short lifetime $(1.5 \mathrm{~ns})$ which has a small amplitude $(<$ $10 \%)$ that we fix as a constant parameter in the fit. From the results of previous bulk 


\section{\begin{tabular}{l|l} 
Chapter 3 & FORCE SENSOR CHARACTERIZATION
\end{tabular}}

Table 3.1.: Lifetime of different sensor strands

\begin{tabular}{ll}
\hline Sensor strands & Lifetimes (ns) \\
\hline $\mathrm{F}(2 \mu \mathrm{M})$ & $3.51 \pm 0.05$ \\
$\mathrm{~F}+\mathrm{H}(2 \mu \mathrm{M}+2 \mu \mathrm{M})$ & $3.66 \pm 0.07$ \\
$\mathrm{~F}+\mathrm{H}+\mathrm{C}(2 \mu \mathrm{M}+2 \mu \mathrm{M}+20 \mu \mathrm{M})$ & $4.03 \pm 0.05$ \\
\hline
\end{tabular}

fluorescence for structural quenching (sec 3.2.4 and the current one, we can conclude that there is the likelihood of guanine quenching the fluorophore in the absence of $\mathrm{Q}$ strands in the sensor. This scenario is completely absent when the hairpin is opened $(\mathrm{F}+\mathrm{H}+\mathrm{C})$. This guanine quenching may arise from the presence of guanine-cytosine (GC) rich bases in the DNA sensor which was designed specifically in order to have the sensors operate at higher forces [138], [67].

\subsubsection{Finding the Efficient Quenching stoichiometry}

DNA Buffer

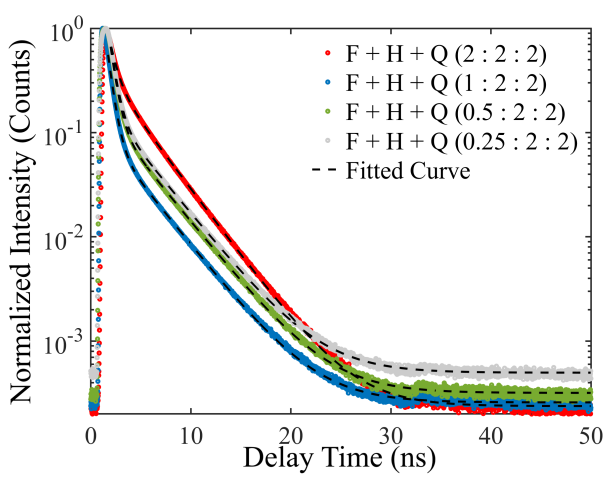

Actin Buffer

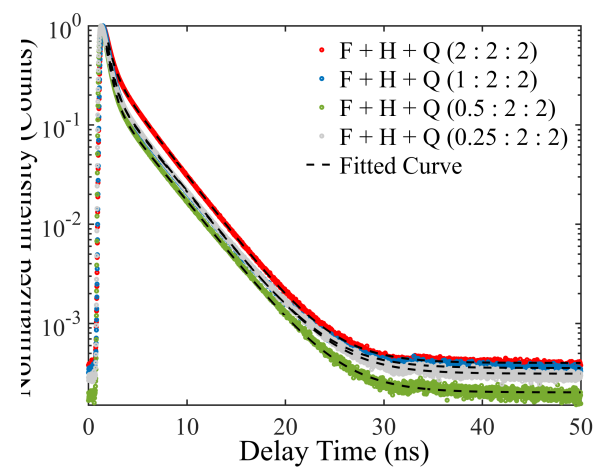

Figure 3.8.: Lifetime decay curves of sensors with decreasing $F$ strand concentration. $F$ strand concentration is decreased step-wise to find the best quenching stoichiometry. $F+H+Q$ is notated as $(2: 2: 2)$ which stands for $(2 \mu \mathrm{M}+2 \mu \mathrm{M}+2 \mu \mathrm{M})$ molar concentration of all strands. $1: 2: 2=1 \mu \mathrm{M}+2 \mu \mathrm{M}+2 \mu \mathrm{M}, 0.5: 2: 2=0.5 \mu \mathrm{M}+2 \mu \mathrm{M}+2 \mu \mathrm{M}$ and $0.25: 2: 2=0.25 \mu \mathrm{M}+2 \mu \mathrm{M}+2 \mu \mathrm{M}$. Left: Measurements in DNA buffer. Right: Measurements in actin buffer.

We began FLIM measurements on DNA sensors by using all strands $(\mathrm{F}, \mathrm{H}, \mathrm{Q})$ in equimolar concentration to find the best efficient quenching stoichiometry. This was done by reducing the concentration of $\mathrm{F}$ strand in sensors, while maintaining the same molar concentration for $\mathrm{H}$ and $\mathrm{Q}$ strands. $\mathrm{C}$ strand was used 10 times higher throughout the thesis to ensure opening of all existing assemblies. The notation $\mathrm{F}+\mathrm{H}+\mathrm{Q}$ in Fig. 3.8 represents the molar concentration stoichiometry of strands with respect to each other. Hence in this experiment i.e. 2:2:2 stands for $2 \mu \mathrm{M}: 2 \mu \mathrm{M}: 2 \mu \mathrm{M}$. It holds good for 1:2:2, 0.5:2:2 and 0.25:2:2. Thus, we titrated for $\mathrm{Q}$ by decreasing the $\mathrm{F}$ concentration in the quenched sensor. Each of 
these stochiometries were individual samples and separate measurements in DNA and in actin buffer. We used a bi-exponential decay function to fit the tail of the histogram (0.5 ns after the maximum) using the same maximum likelihood procedure. The fitting function in this case was

$$
I(t)=\frac{A_{1}}{\tau_{1}} e^{-t / \tau_{1}}+\frac{A_{2}}{\tau_{2}} e^{-t / \tau_{2}}+b
$$

Where $\tau_{1}$ and $\tau_{2}$ are fluorescence lifetimes and $A_{1}$ and $A_{2}$ are respective amplitudes and $\mathrm{b}$ is the background.

By fitting the exponential function in Eqn 3.2 we obtain two lifetimes $\tau_{1}$ and $\tau_{2}$ for the decay curves of quenched sensors in Fig. 3.8. This signifies that two populations of sensors exist (from two amplitudes $A_{1}$ and $A_{2}$ from Eqn 3.2), a quenched and non-quenched population. Quenched sensors have lifetimes around $0.5 \mathrm{~ns}$ and the second non-quenched lifetimes are around $3.5 \mathrm{~ns}$. Their corresponding populations are denoted in a bracket in Table 3.2. The best efficient quenching in DNA buffer is seen for two stoichiometries, 0.5:2:2 (38\%) \& 0.25:2:2 (42\%). However, in actin buffer, 0.5:2:2 stoichiometry yields the best quenching $(28 \%)$. Lifetimes remain the same in both buffers for all stoichiometries. The non-quenched sensors obtained from $\tau_{2}$, is a large population of around $60 \%-83 \%$ (given by long lifetimes 3.3 - $3.5 \mathrm{~ns}$ ). These sensors may have a conformation that is either unassembled or may possess a partial assembly $(\mathrm{F}+\mathrm{H})$.

Table 3.2.: Lifetime of quenched sensors (FHQ) with decreasing F strand concentration

\begin{tabular}{lll}
\hline F:H:Q $(\mu \mathrm{M})$ & $\tau_{1}(\mathrm{~ns})$ & $\tau_{2}(\mathrm{~ns})$ \\
\hline DNA buffer & & \\
$2: 2: 2$ & $0.56 \pm 0.04(23 \%)$ & $3.58 \pm 0.07(77 \%)$ \\
1:2:2 & $0.52 \pm 0.04(35 \%)$ & $3.65 \pm 0.06(65 \%)$ \\
$0.5: 2: 2$ & $0.51 \pm 0.08(38 \%)$ & $3.63 \pm 0.07(62 \%)$ \\
$0.25: 2: 2$ & $0.50 \pm 0.08(42 \%)$ & $3.58 \pm 0.08(58 \%)$ \\
Actin buffer & & \\
2:2:2 & $0.63 \pm 0.08(17 \%)$ & $3.38 \pm 0.07(83 \%)$ \\
1:2:2 & $0.57 \pm 0.02(24 \%)$ & $3.52 \pm 0.03(76 \%)$ \\
$0.5: 2: 2$ & $0.56 \pm 0.04(28 \%)$ & $3.51 \pm 0.05(72 \%)$ \\
$0.25: 2: 2$ & $0.58 \pm 0.05(24 \%)$ & $3.52 \pm 0.05(76 \%)$ \\
\hline
\end{tabular}

Additionally as an alternative analysis, for the bi-exponential fit, we kept the longer lifetime value $\tau_{2}$ fixed (non-quenched population of fluorophores). This we did, to see if there will be a further drastic increase in the quenched population (population of $\tau_{1}$ ). What we observe is that the long lifetime value that is fixed here is the same lifetime obtained for controls in its closed state $\left(\mathrm{FHQ}^{-}\right)$which indicates that quencher strands are not present in a configuration to quench. Table 3.3 gives the lifetimes of quenched sensors when the long lifetime is fixed. It can be observed that there is no significant increase 


\section{\begin{tabular}{l|l} 
Chapter 3 & FORCE SENSOR CHARACTERIZATION
\end{tabular}}

Table 3.3.: Lifetime of quenched sensors (FHQ) with decreasing $F$ strands when $\tau_{2}=$ a constant

\begin{tabular}{lll}
\hline F:H:Q $(\mu \mathrm{M})$ & $\tau_{1}(\mathrm{~ns})$ & $\tau_{2}(\mathrm{~ns})$ \\
\hline DNA buffer & & \\
$2: 2: 2$ & $0.62 \pm 0.04(25 \%)$ & $3.70 \pm 0.07(75 \%)$ \\
$1: 2: 2$ & $0.53 \pm 0.04(36 \%)$ & $3.70 \pm 0.06(64 \%)$ \\
$0.5: 2: 2$ & $0.53 \pm 0.08(38 \%)$ & $3.70 \pm 0.07(62 \%)$ \\
$0.25: 2: 2$ & $0.51 \pm 0.08(43 \%)$ & $3.70 \pm 0.08(57 \%)$ \\
Actin buffer & & \\
$2: 2: 2$ & $0.80 \pm 0.08(22 \%)$ & $3.58 \pm 0.07(78 \%)$ \\
$1: 2: 2$ & $0.59 \pm 0.02(25 \%)$ & $3.58 \pm 0.03(75 \%)$ \\
$0.5: 2: 2$ & $0.59 \pm 0.04(29 \%)$ & $3.58 \pm 0.05(71 \%)$ \\
$0.25: 2: 2$ & $0.61 \pm 0.05(29 \%)$ & $3.58 \pm 0.05(71 \%)$ \\
\hline
\end{tabular}

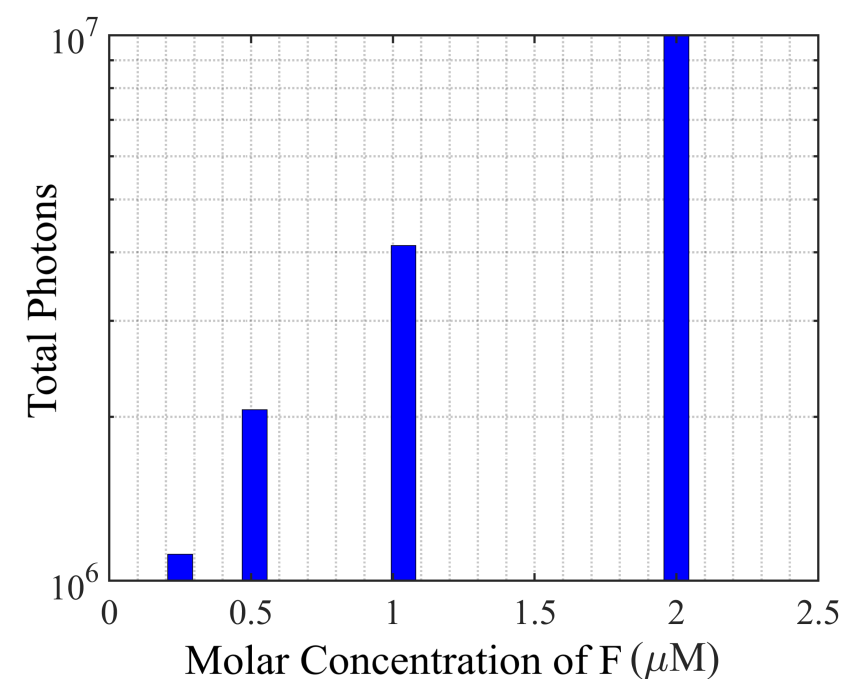

Figure 3.9.: Total photon counts of quenched sensors for decreasing concentration of $F$ strand. The total fluorescence intensity of quenched sensors for their decreasing concentration of $F$ strand is shown here. Total amount of photons represents the quenched photons and non quenched (long lifetime) photons.

(only 1-2\%) in the population of quenched sensors in both buffers. Lifetimes statistically remain the same between the former and this current method of analysis (fixing the long lifetime). That is, the non-quenched population remains the same by whichever means of analysis. Figure 3.9 is depicted to show the decrease in total fluorescence (quenched \& non-quenched photons) as F concentration in sensor is decreased.

From this experiment, we optimize and will further use 1:2:2 $(1 \mu \mathrm{M}: 2 \mu \mathrm{M}: 2 \mu \mathrm{M})$ as the most efficient quenched stoichiometry in both buffers for all future experiments. In this 
stoichiometry, DNA buffer gives a $35 \%$ quenched population while in actin $24 \%$ population of sensors are quenched. This huge variation may arise due to the different salt conditions ( buffer compositions given in A.1, A.2) that might affect the stability of the hairpins in sensors which is further detailed under discussion in 3.3 .8 .

\subsubsection{FLIM on Quenched and Opened Sensors}

DNA Buffer

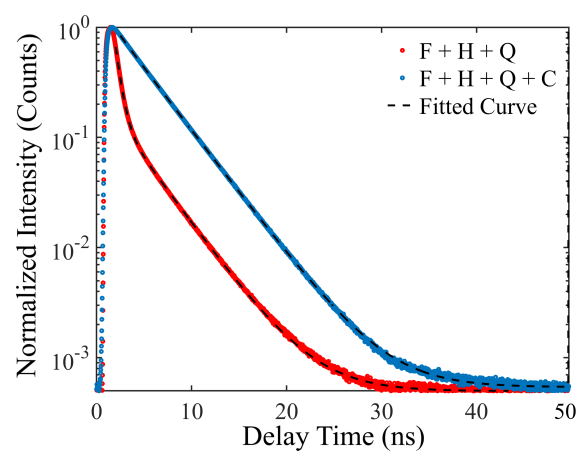

Actin Buffer

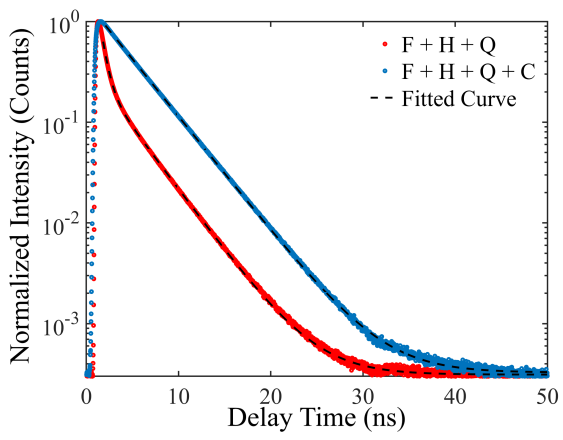

Figure 3.10.: Quenched and opened sensors lifetime decay curves in DNA and actin buffers. The lifetime decay curves of quenched sensors (red curve) have a bi-exponential component decay. The short slightly bent region (red curve) represents the quenching of sensors due to a high FRET. Blue curves represent the opened sensors that have a long lifetime decay due to a low FRET. Decay curves of sensors in DNA buffer (left) and actin buffer (right). $F+H+Q$ : Quenched sensors. $\mathrm{F}+\mathrm{H}+\mathrm{Q}+\mathrm{C}$ : Opened sensors.

Table 3.4.: Lifetime of DNA sensors when quenched $(F+H+Q)$ and opened $(F+H+Q+C)$ in DNA and actin buffer

\begin{tabular}{lll}
\hline Sensor strands & $\tau_{1}(\mathrm{~ns})$ & $\tau_{2}(\mathrm{~ns})$ \\
\hline DNA buffer & & \\
$\mathrm{F}+\mathrm{H}+\mathrm{Q}$ & $0.52 \pm 0.04(35 \%)$ & $3.65 \pm 0.06(65 \%)$ \\
$\mathrm{F}+\mathrm{H}+\mathrm{Q}+\mathrm{C}$ & $3.81 \pm 0.03(100 \%)$ & \\
Actin buffer & & \\
$\mathrm{F}+\mathrm{H}+\mathrm{Q}$ & $0.57 \pm 0.02(24 \%)$ & $3.52 \pm 0.03(76 \%)$ \\
$\mathrm{F}+\mathrm{H}+\mathrm{Q}+\mathrm{C}$ & $3.70 \pm 0.09(100 \%)$ & \\
\hline
\end{tabular}

In this section, we compare the differences in lifetime between the quenched sensor and the opened sensor (for the optimized stoichiometry 1:2:2). The lifetime decay curves with their corresponding lifetime for the quenched and open states establish very clearly the working of sensors via FRET. These measurements were made in both buffers as shown in Fig. 3.10. The decay curves for opened sensors were fit with monoexponential function 


\section{\begin{tabular}{l|l} 
Chapter 3 & FORCE SENSOR CHARACTERIZATION
\end{tabular}}

(Eqn 3.1) and their respective lifetime, amplitude (population) are given in Table 3.4 In both buffers, a prominent quenching is observed given by the bent region (in the red curve) which is quite long for the DNA buffer than for the actin buffer. It implies that a large population of sensors is quenched (35\%) in DNA buffer than in actin buffer $(24 \%)$. When opened, sensors posses a single lifetime (blue decay curves) in both buffers. Opened sensor lifetimes are slightly less in actin buffer (3.70 $\pm 0.09 \mathrm{~ns})$ than in DNA buffer (3.81 \pm $0.03 \mathrm{~ns})$. We observe a $(100 \%)$ population of a long lifetime for the opened sensors which shows that all sensors are completely opened in both buffers. To conclude, lifetimes are similar in both buffers, more quenched population is observed in DNA buffer, and all sensors are completely opened when $\mathrm{C}$ strand is added. At zero force, the sensor lifetime is $0.5 \mathrm{~ns}$ and for a threshold opening force the lifetime is $3.6 \mathrm{~ns}$.

\subsubsection{FLIM on Controls in Closed and Open Position}

DNA Buffer

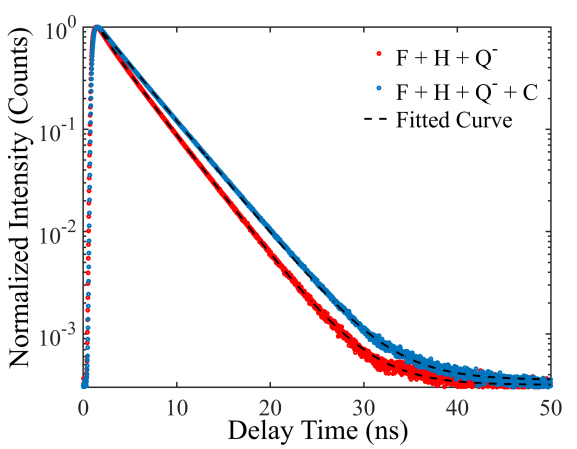

Actin Buffer

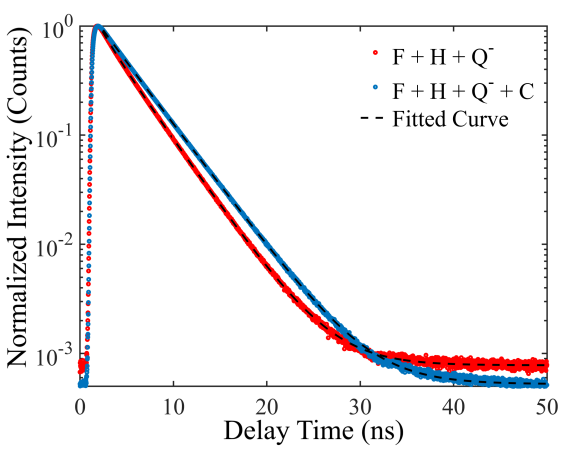

Figure 3.11.: Lifetime decay curves of controls. The fluorescence decay curves of controls in their closed $\left(\mathrm{F}+\mathrm{H}+\mathrm{Q}^{-}\right)$and open state $\left(\mathrm{F}+\mathrm{H}+\mathrm{Q}^{-}+\mathrm{C}\right)$ is represented. Both show a monoexponential decay with a long lifetime due to the absence of a quencher on the $Q$ strand. Left: Measurements in DNA buffer. Right: Measurements in actin buffer.

Table 3.5.: Lifetime of controls in DNA and actin buffer

\begin{tabular}{ll}
\hline Controls & $\tau(\mathrm{ns})$ \\
\hline DNA buffer & \\
$\mathrm{F}+\mathrm{H}+\mathrm{Q}^{-}$ & $3.70 \pm 0.10$ \\
$\mathrm{~F}+\mathrm{H}+\mathrm{Q}^{-}+\mathrm{C}$ & $3.94 \pm 0.07$ \\
Actin buffer & \\
$\mathrm{F}+\mathrm{H}+\mathrm{Q}^{-}$ & $3.58 \pm 0.08$ \\
$\mathrm{~F}+\mathrm{H}+\mathrm{Q}^{-}+\mathrm{C}$ & $3.86 \pm 0.09$ \\
\hline
\end{tabular}


The bulk fluorescence of controls in their closed and open positions in DNA buffer was quantified with a spectrometer as discussed in Chapter $3(\sec 3.2 .3$ and in Fig. 3.5. In this section, their fluorescence lifetime along with their decay curves for these two positions will be discussed. Since the quencher is absent, no FRET occurs, therefore we expect a single longer lifetime for the fluorophore (Alexa 488). Controls lifetime, like sensors were also measured in two buffers (DNA and actin buffer). As depicted in Fig. 3.11 they have a complete mono-exponential decay with a long lifetime (observed as a straight curve) for closed and open positions. Their respective lifetime values obtained by a mono-exponential fitting (Eqn 3.1) are given in Table 3.5. The closed position controls (red curves) in both buffers decay with a slightly lower lifetime ( $3.70 \pm 0.10 \mathrm{~ns} \& 3.5 \pm$ $0.08 \mathrm{~ns})$ than the open position control ( $3.94 \pm 0.07 \mathrm{~ns} \& 3.86 \pm 0.09 \mathrm{~ns}$, blue curves). This lower lifetime for the closed position controls validates further the presence of structural quenching that is seen even in the absence of quencher which has been previously discussed under structural quenching (section 3.3.2.

\subsubsection{PCR Annealing of Sensors Distorts Quenching}

\section{PCR Annealed Sensor and Controls}

\section{$2: 2: 2$}

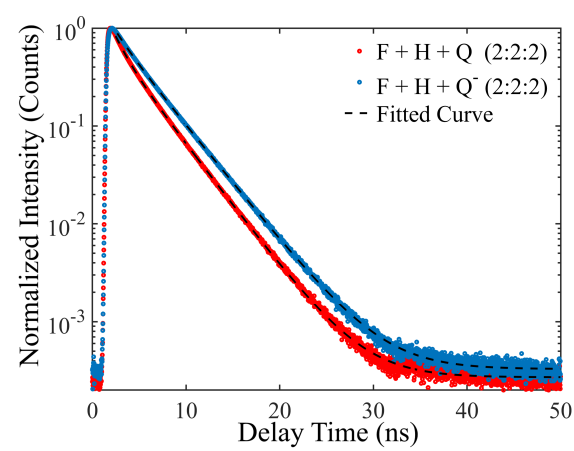

$1: 2: 2$

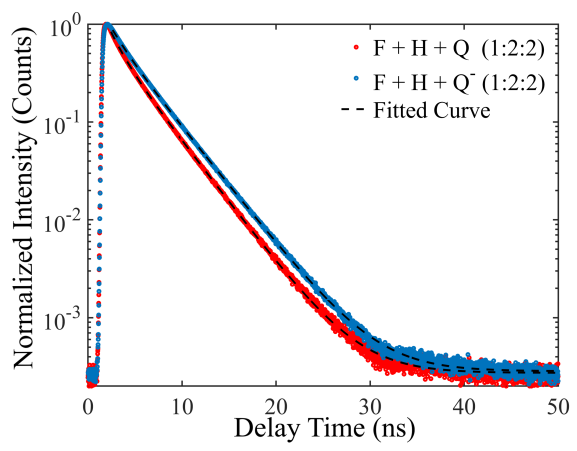

Figure 3.12.: PCR annealed sensors and controls lifetime measurements. Lifetime decay curves of annealed sensors and annealed controls. $P C R$ annealed quenched sensors $(F+H+Q$, red curve) show a completely non-quenched lifetime decay curve similar to the controls $\left(\mathrm{F}+\mathrm{H}+\mathrm{Q}^{-}\right.$, without quencher, blue curve). Due to the annealing of quenched sensors in PCR, they do not have their characteristic bi-exponential lifetime that has a short bent region in its decay curve. $\mathrm{F}: \mathrm{H}: \mathrm{Q}$ denotes the molar concentrations of each strand. $2: 2: 2$ is the $2 \mu \mathrm{M}+2 \mu \mathrm{M}+2 \mu \mathrm{M}$ and $1: 2: 2$ stands for $1 \mu \mathrm{M}+2 \mu \mathrm{M}+2 \mu \mathrm{M}$.

To remove mismatches and obtain a proper strand hybridization, sensors and controls were annealed in a PCR instrument by heating to $95^{\circ} \mathrm{C}$ and gradually cooling to $20^{\circ} \mathrm{C}$. We then performed FLIM on these samples for the 2:2:2 (F:H:Q) and 1:2:2 (F:H:Q) concentrations. From the fluorescence decay curves in Fig. 3.12, there is clear evidence that quenching does not occur in the PCR annealed sensor. Generally, quenched sensors (that 


\section{\begin{tabular}{l|l} 
Chapter 3 & FORCE SENSOR CHARACTERIZATION
\end{tabular}}

are room temperature annealed), exhibit decay curves with a bi-exponential component, which is seen as the small bent region in the first few nanoseconds of the decay curves (Fig. 3.8. We do not observe this bent region (quenching) in the decay curves for PCR annealed sensors at both the concentrations, 2:2:2 $(2 \mu \mathrm{M}: 2 \mu \mathrm{M}: 2 \mu \mathrm{M})$ and 1:2:2 $(1 \mu \mathrm{M}: 2 \mu \mathrm{M}: 2 \mu \mathrm{M})$. Instead, we find the opposite behavior. A completely mono-exponential longer lifetime are seen (blue curves in Fig. 3.12). Upon fitting these PCR annealed sensor decay curves, a reduced lifetime of $1.26 \mathrm{~ns}$ for (2:2:2) and 1:25 ns for (1:2:2) is observed and not a quenched lifetime (Table 3.6). On the other hand, controls that were also PCR annealed, shows no change in lifetime but represent their usual longer lifetime of $3.63 \mathrm{~ns}$ for (2:2:2) and 3.69 ns for $(1: 2: 2)$ similar to the room temperature annealed controls which is $3.70 \pm 0.10 \mathrm{~ns}$ for 1:2:2 (Table 3.5).

Table 3.6.: Lifetimes of annealed DNA sensors and annealed controls

\begin{tabular}{lll}
\hline Strands $(\mu \mathrm{M})$ & $\tau_{1}(\mathrm{~ns})$ & $\tau_{2}(\mathrm{~ns})$ \\
\hline Annealed sensors & & \\
$\mathrm{F}+\mathrm{H}+\mathrm{Q}(2: 2: 2)$ & $1.26 \pm 0.05(14 \%)$ & $3.46 \pm 0.07(86 \%)$ \\
$\mathrm{F}+\mathrm{H}+\mathrm{Q}(1: 2: 2)$ & $1.25 \pm 0.06(14 \%)$ & $3.46 \pm 0.06(86 \%)$ \\
Annealed controls & & \\
$\mathrm{F}+\mathrm{H}+\mathrm{Q}^{-}(2: 2: 2)$ & $3.63 \pm 0.04(89 \%)$ & \\
$\mathrm{F}+\mathrm{H}+\mathrm{Q}^{-}(1: 2: 2)$ & $3.69 \pm 0.07(92 \%)$ & \\
\hline
\end{tabular}

Gel electrophoresis experiments supports the above observations. The PCR annealed sensors were annealed in a PCR instrument through the heating and cooling cycle as mentioned in Table A.4 The room temperature annealed sensors were prepared by mixing the strands together. Both were loaded on 20\% TBE gels in PAGE (Polyacrylamide gel electrophoresis) as shown in Fig. 3.13 and were stained with SYBR Gold, which stains the single and double-stranded DNA. Hence, the presence of single strands is also visible in the gel electrophoresis. These were later imaged under UV fluorescence. Several combinations of different strands such as FH, FHC, FHQ, FHQC, QC, QHC, HC were prepared in order to check for hybridization and improper assembly. Individual strands of sensors $(\mathrm{F}, \mathrm{H}, \mathrm{Q}, \mathrm{C})$ were also run in the same gel.

From the SYBR staining of sensors, we observe a very faint fluorescence for the PCR annealed sensor (FHQ) in Fig. 3.13 indicated by the red arrow. The room temperature annealed sensor (FHQ) shows a bright fluorescence indicating the existence of more amount of assembled sensors. This experiment support our lifetime measurements, where sensors, when annealed in a PCR, showed a non-quenched lifetime (Table A.4 which in this electrophoresis experiment is seen as faint fluorescence band. Both of these results point to the presence of a poorly assembled sensor when annealed in a PCR experiment. Another observation is that whether sensors were PCR or room temperature annealed, the 

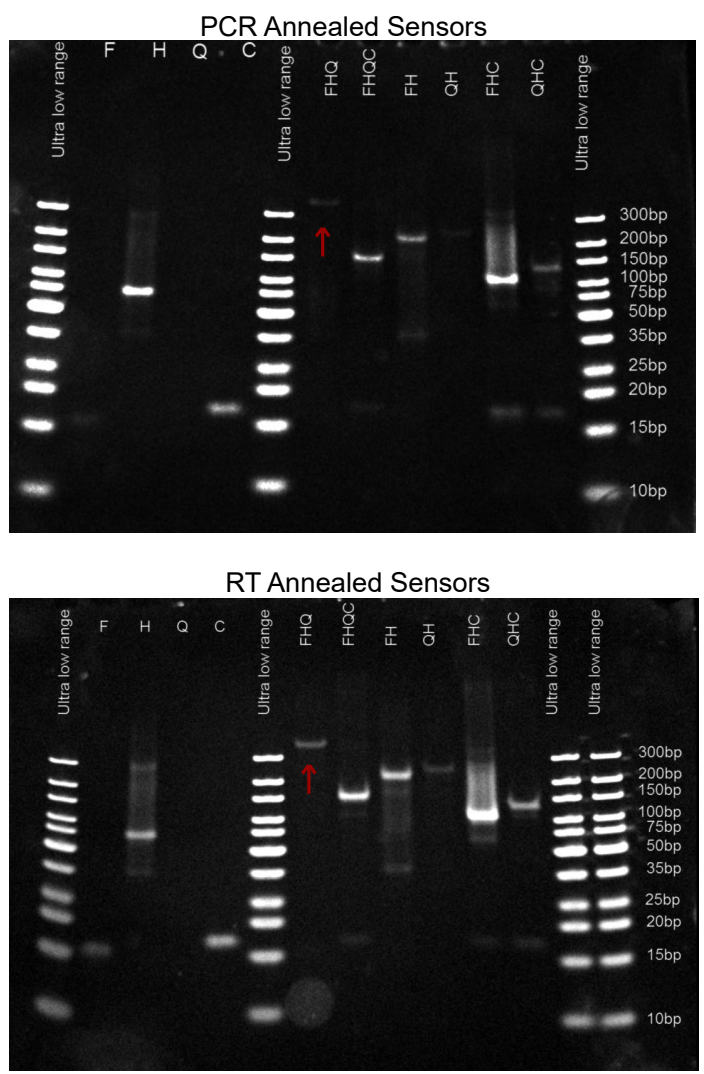

Figure 3.13.: PCR and room temperature annealed sensors. DNA sensors with several combinations of strand assemblies annealed in PCR and at room temperature in PAGE electrophoresis. FHQ, FHQC, FH, FHC, QC, QHC, HC are the combinations of strand assemblies. FHQ depicts a quenched sensor. PCR annealed sensor (FHQ, top image, red arrow) shows a faint fluorescence in SYBR gold staining due to poor assembly of quenched sensors. Room temperature annealed sensor (FHQ, bottom image, red arrow) shows a bright fluorescence indicating the presence of a fully assembled sensor in the quenched state. PCR Annealing thus distorts the assembly of DNA in the quenched state. The ultra-low range represents the ladder. Sensor concentration for annealing: $2 \mu \mathrm{M}$. Sensor concentration for gel electrophoresis: $250 \mathrm{nM}$.

quenched and opened states of DNA sensors can be visualized from these gels. Quenched (FHQ) and open (FHQC) appear at different locations in the gel. Quenched sensors (FHQ) that are both PCR and room temperature annealed, are seen at the top of the gel (band observed above $300 \mathrm{bp}$ in Fig. 3.13) as the mobility of FHQ is slow in electrophoresis similar to the mobility of a coiled or ring DNA. FHQC, the opened sensors (band observed at 150 bp in Fig. 3.13) are stretched out, runs faster in gel electrophoresis like a linear DNA. All other assemblies (FH, QH, FHC, QHC) are not fully assembled/coiled as they lack one strand of DNA sensor. Therefore their mobilites are faster and are observed above or below the the opened sensor (FHQC) in Fig. 3.13 


\section{\begin{tabular}{l|l} 
Chapter 3 & FORCE SENSOR CHARACTERIZATION
\end{tabular}}

\subsubsection{FRET Efficiency of DNA Sensors}
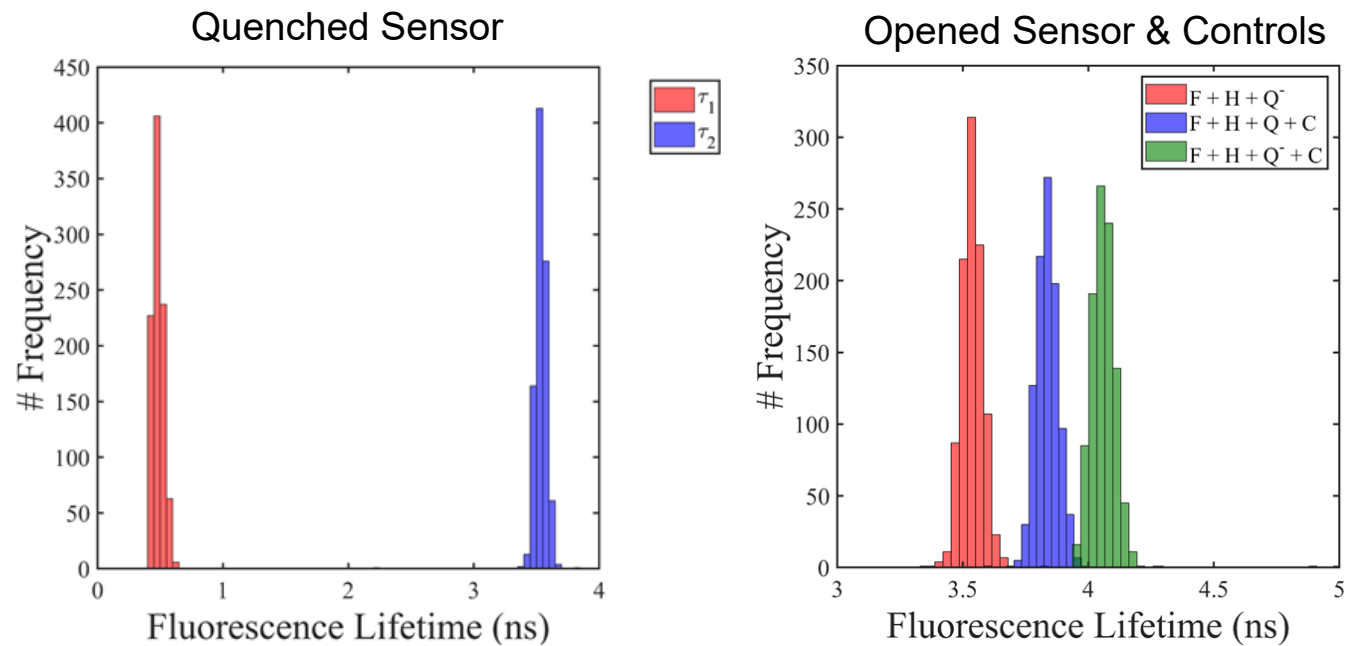

Figure 3.14.: Distribution of quenched and fluorescence lifetime of sensors. Left side: Quenched sensors have two different lifetimes, a quenched short lifetime of 0.5 ns and a nonquenched long lifetime of 3.5 ns (in blue). Right side: All the fluorescent states i.e. opened sensors $(\mathrm{F}+\mathrm{H}+\mathrm{Q}+\mathrm{C})$ in blue with $3.9 \mathrm{~ns}$, closed controls $\left(\mathrm{F}+\mathrm{H}+\mathrm{Q}^{-}\right)$in orange with $3.5 \mathrm{~ns}$ and opened controls $\left(\mathrm{F}+\mathrm{H}+\mathrm{Q}^{-}+\mathrm{C}\right)$ in green with $4.1 \mathrm{~ns}$.

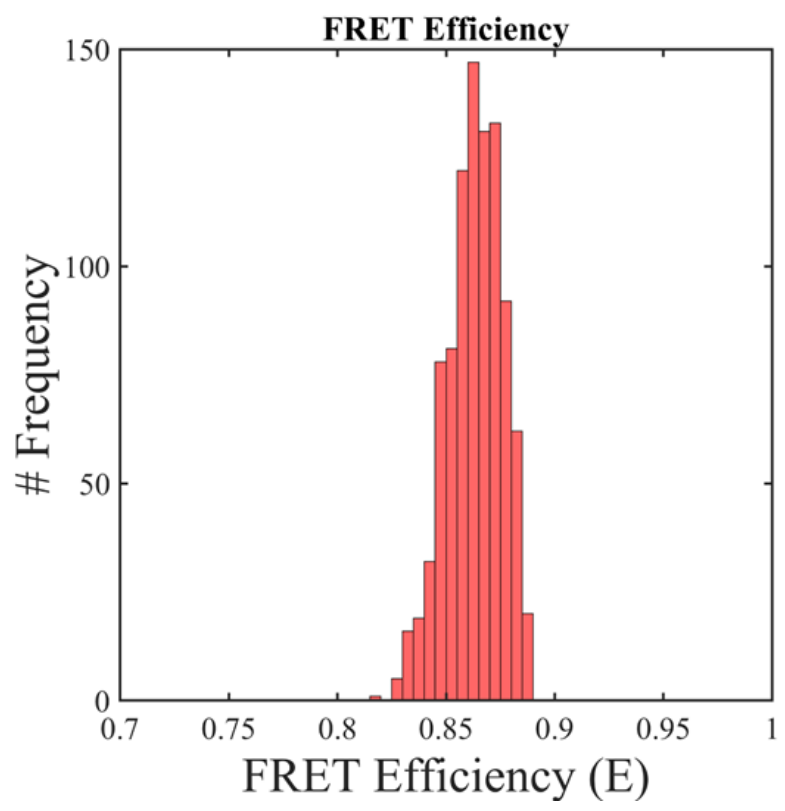

Figure 3.15.: FRET efficiency of DNA sensors. Molecular DNA force sensors are 85\% FRET efficient which is estimated from the lifetime of the quenched sensor $\left(\tau_{D A}\right)$ and closed controls $\left(\tau_{D}\right)$. 
Lifetime obtained from FLIM measurements is represented in Fig. 3.14 for quenched sensors on the left and for all the fluorescent states (opened sensor, closed controls, opened controls) on the right. Quenched sensors show two distinct lifetimes, a quenched lifetime around $0.6 \mathrm{~ns}$ and a long lifetime population of $3.7 \mathrm{~ns}$ which is depicted as a histogram on the left side image in Fig. 3.14 All fluorescence states are governed by a single long lifetime as represented on the right side image in Fig. 3.14 Opened sensors $(\mathrm{F}+\mathrm{H}+\mathrm{Q}+\mathrm{C})$ have a long lifetime around $3.8 \mathrm{~ns}$, closed controls $\left(\mathrm{F}+\mathrm{H}+\mathrm{Q}^{-}\right)$around $3.5 \mathrm{~ns}$ and opened controls $\left(\mathrm{F}+\mathrm{H}+\mathrm{Q}^{-}+\mathrm{C}\right)$ around $4.1 \mathrm{~ns}$. After the determination of lifetimes for senors and controls, we estimated the FRET efficiency of our sensors according to the equation.

$$
E=1-\frac{\tau_{D A}}{\tau_{D}}
$$

$\tau_{D A}$ - quenched sensor lifetime, $\tau_{D}$ - closed controls lifetime.

The FRET efficiency of our DNA sensor is 0.85 (given in Fig. 3.15). In other words, our molecular DNA force sensors have a $85 \%$ FRET efficiency, which is a high efficiency for a FRET based sensor.

\subsubsection{Discussion}

In this chapter, we have extensively characterized the sensors efficiency by bulk fluorescence measurements (with a spectrometer) and by lifetime measurements via FLIM. In the spectrometer, the quenched and high fluorescence of sensors were quantified, in solutions (DNA and actin buffer) and while crosslinked to actin. Fluorescence of 'controls' that is unchanged at zero force (closed) or at threshold opening force (opened state) proves that our sensor works via FRET.

Experiments that involved the addition of $C$ strand to different lengths of the hairpin (16 bp \& $24 \mathrm{bp}$ ) proves the energy-based opening for the hairpin 8 (stem length - $8 \mathrm{bp}$ ) via its $C$ strand. In a hairpin structure, the properties of the stem (stem length, GC content) governs its unfolding free energy $(\Delta G)$ and its behavior [137], [138]. From these reports it can be seen that the unfolding force of hairpins rises linearly with increased stem length and for increased GC content.Thus, in our DNA sensors unfolding of hairpins with higher stem lengths such as $16 \mathrm{bp}$ and $24 \mathrm{bp}$ was not possible with the help of a C strand designed for hairpin 8 . It proves again that unfolding of hairpins can be tuned by varying the length of hairpins.

The calculated opening threshold force for our DNA sensors with hairpin stem length 8 bp (69.4\% GC content) is around $10 \mathrm{pN}$ which has a lifetime (DNA buffer) of 3.6 ns. At 0 $\mathrm{pN}$ (folded state, quenched) the lifetime is $0.5 \mathrm{~ns}$. The opening threshold force of our DNA sensor is an estimated value that we use from reports that have calculated the threshold 


\section{\begin{tabular}{l|l} 
Chapter 3 & FORCE SENSOR CHARACTERIZATION
\end{tabular}}

force for hairpins with varying stem length and GC content [137], [138]. Our DNA sensors offer the possibility of being easily tuned for different force ranges by changing its stem length (as stated above $8 \mathrm{bp}, 16 \mathrm{bp} \& 24 \mathrm{bp}$ ), GC content and temperature that has also been previously reported in various works [104], [136], [137].

Structural quenching is prominently observed in the $\mathrm{F}, \mathrm{F}+\mathrm{H}$ and $\mathrm{F}+\mathrm{H}+\mathrm{C}$ strands in fluorescence and FLIM measurements. The reduction in fluorescence and lifetimes in $\mathrm{F}$ and $\mathrm{F}+\mathrm{H}$ strands which disappears completely when opened by $\mathrm{C}$ strands is an indication that quenching from the base sequences of DNA occurs. Quenching of fluorophore by guanine and guanosine nucleotides if present right next to the fluorophore has been previously reported [52], [112], [78], [57], [103], [93]. Noble et al., [97] have reported the quenching of Alexa 488 dye by guanosine to be dependent on the position, number of guanosine bases and the site of fluorophore attachment in the oligonucleotide strand. In our case, we have a cytosine residue right next to the fluorophore in the F strand and a guanine base on the $\mathrm{H}$ strand closer to the fluorophore which could possibly lead to this quenching due to photo-electron energy transfer as reported in [78], [112].

The lifetime of DNA sensors and controls characterized by FLIM measurements portrays excellently the quenched (zero force) and open (threshold force) states from their characteristic lifetimes. The population of quenched sensors decreases in the actin buffer which could possibly be due to its varied salt concentration as it has a low concentration of divalent salt ( $2 \mathrm{mM} \mathrm{Mgcl}_{2}$ ), which might affect the stability of the hairpin. Since nucleic acid structures are polyanionic in nature, the influence of metal ions play an important role in their stability. It has been shown that loop stability in hairpins is dependent on ion concentration [121].

Annealing of sensors in PCR distorts their ability to quench via FRET. There was no observed quenched lifetime but rather a reduced lifetime of around $1.25 \mathrm{~ns}$ and a long lifetime of around $3.5 \mathrm{~ns}$ when sensors were annealed in a PCR. The electrophoresis (PAGE) experiment also supports these lifetime results where a faint fluorescent band is observed for FHQ (quenched sensors) when annealed in the PCR whereas a bright fluorescent band is observed for sensors annealed at room temperature. Thus upon PCR annealing, sensors are present in a configuration where they do not quench via FRET.

Lifetime measurements enabled us to finally determine the FRET efficiency of our DNA sensors as $85 \%$. This is a very high efficiency of FRET among force sensors that are based on FRET pairs. As an example, a well defined existing DNA force sensor has an efficiency of around $79 \%$ [113]. 


\section{DNA Sensors in In Vitro Actin Network \& in Cellular Actin}

\subsection{DNA Sensors Crosslinked to Actin (In Vitro)}

This chapter deals with the attachment strategy of sensors to the cytoskeletal component - actin and characterization of DNA sensors via the FLIM technique while they are crosslinked to actin filaments. Sensors in vitro refers to their usage as crosslinkers of actin filaments. These sensors were also introduced into live 3T3 fibroblasts where they were attached to cellular actin via lifeact. We used FLIM to characterize sensors in in vitro networks and cells. In both of these cases, sensors need to be attached to actin. To achieve this haloTag fusion protein technology [70] was used. The outer ends of sensors were modified and a haloligand was incorporated. A covalent bond is formed between the haloligand and the haloTag. The haloTag is first genetically fused to RFP lifeact. Thus, sensors attach to actin via the lifeact which is an actin-binding peptide [106]. A pictorial representation of this attachment strategy is given in Fig. 4.1. By this, the in vitro quenched and opened sensors (with the $\mathrm{C}$ strand) can be crosslinked to actin filaments. Controls were also crosslinked to actin in the same manner, and lifetime measurements were performed. The optimized F:H:Q stoichiometry, 1:2:2 (1 $\mathrm{MM}: 2 \mu \mathrm{M}: 2 \mu \mathrm{M})$ from (sec 3.3.3) will be referred to as 0.5:1:1 (F:H:Q) throughout this chapter. This notation signifies the ratio of each strand to the other, rather than representing it in terms of molar concentration. 

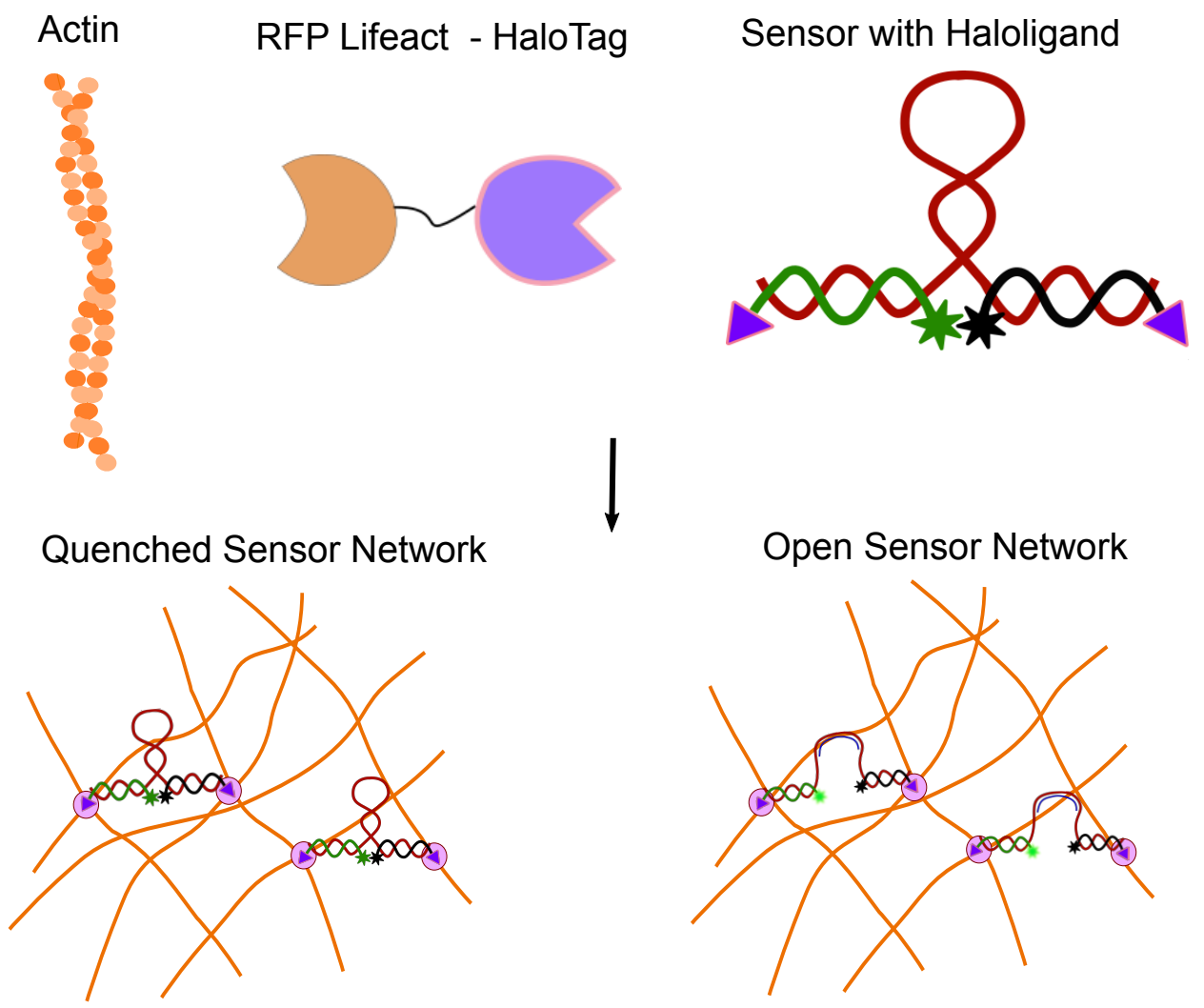

Figure 4.1.: DNA sensor crosslinked across actin network. Attachment strategy of sensors to actin filaments. Top: Actin is attached to sensors via the RFP lifeact haloTag system. The outer ends of $F$ and $Q$ strands in sensors are modified with haloligand (represented as violet triangles) which then covalently binds to haloTag that is genetically coupled to the RFP lifeact. The bottom left shows quenched sensors crosslinked to actin filaments and on the bottom right are opened sensors crosslinked to actin filaments.

\subsubsection{Spectrometer: Bulk Fluorescence of Sensors Crosslinked to Actin}

\section{Working of sensors in actin network}

Figure 4.2 illustrates the demonstration of sensor efficiency while crosslinked to actin in a spectrometer. We mixed F strands to actin quoted as AF (actin \& F). Similarly, Q strands were added to actin denoted as AQ (actin \& Q). The total actin concentration is $0.5 \mathrm{mg} / \mathrm{ml}(11.9 \mu \mathrm{M})$. Both of these mixtures were allowed to polymerize separately. After an hour of polymerization, an emission scan was performed by the sequential addition of each of the sample mixture. AF was first added to DNA buffer which is observed as a high fluorescence signal (green curve). Next, $\mathrm{H}$ strands were added. Finally, after the addition of the AQ mixture, quenching of sensors in the actin network can be observed (black curve). C strand is added which is $20 \mu \mathrm{M}$ (10 times excess in molar concentration than other strands). This was done to open all sensors. Sensors open up across the actin network which is noticed as an increase in fluorescence (blue curve) from the quenched 


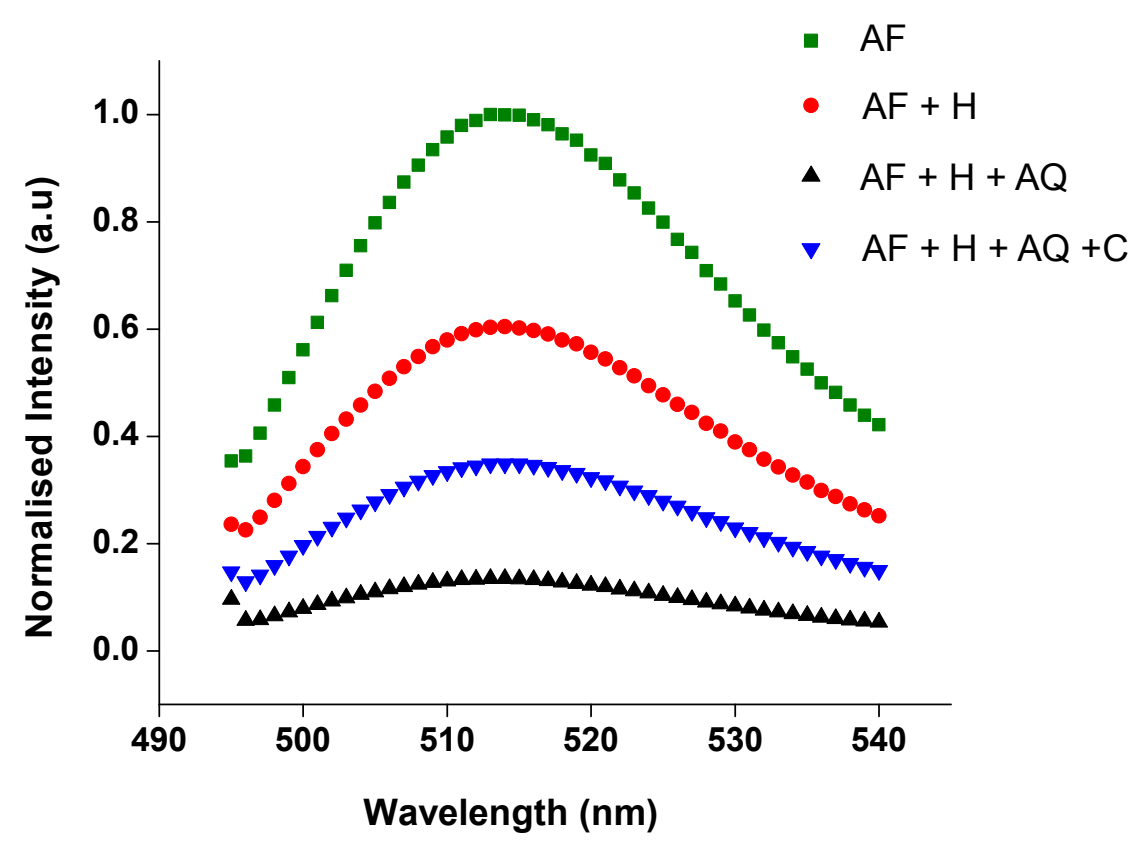

Figure 4.2.: Working of sensors crosslinked to actin. Emission spectra of DNA sensors crosslinked to actin filaments. AF represents actin attached to the $F$ strand of the sensor. $A Q$ is actin crosslinked to the $Q$ strand of the sensor. $A F+H$ represents $H$ strands added to the $A F$ mixture. $A F+H+A Q$ denotes actin attached to $F$ strands $+H$ strands $+Q$ strands attached to actin. Sensors are quenched after $A Q$ was added to the $A F+H$ mixture. Adding $C$ (complementary) strand opens the quenched sensors in the actin network which is seen as an increase in fluorescence.

state. Through this experiment, we quantified the sensors' fluorescence while they are crosslinked to actin. Note: A general observation is that, the fluorescence of sensors in actin network (Fig. 4.2) is slightly lower than sensors in solution - DNA buffer (Fig. 3.3p).

\section{Fluorescence of sensors and controls while crosslinked in an actin network}

Before characterizing the sensors via FLIM, we compare quantitatively in a spectrometer the bulk fluorescence of DNA force sensors and controls while both are crosslinked to actin. F strand in the sensor is half of its molar concentration with respect to $\mathrm{Q}$ and $\mathrm{H}$. This is denoted as 0.5:1:1 (F:H:Q) throughout this chapter. Actin concentration remains the same $(23.81 \mu \mathrm{M}-1 \mathrm{mg} / \mathrm{ml})$ for all conditions and network types unless specified. ActinDNA sensor networks were prepared as described in A.1. They were left undisturbed in the cuvette (Type No. 105.253-QS, Hellma Analytics, Germany) and emission spectral measurements were made an hour after polymerization.

Similar to solution measurements in chapter 3. DNA force sensors while crosslinked to actin also exhibits well pronounced quenched fluorescence (black curve in Fig. 4.33). When they are opened by C strand, a strong increase in fluorescence is observed (green 
a)

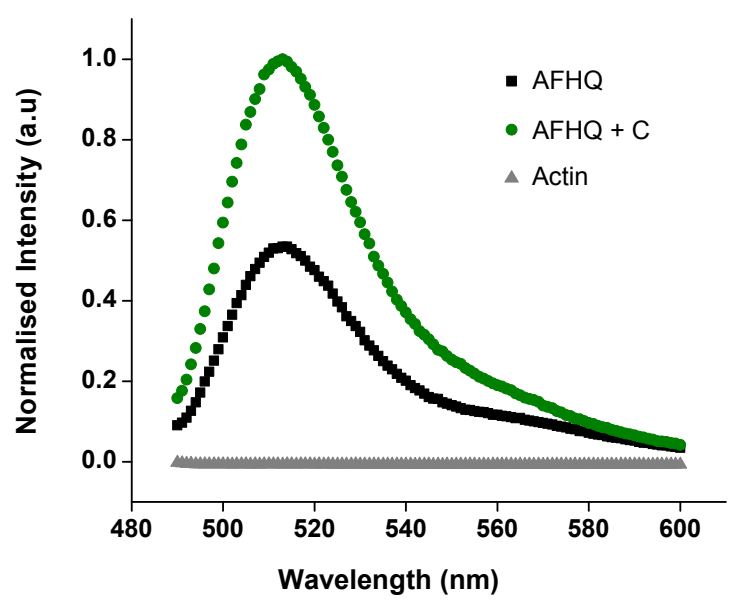

b) Actin + Controls

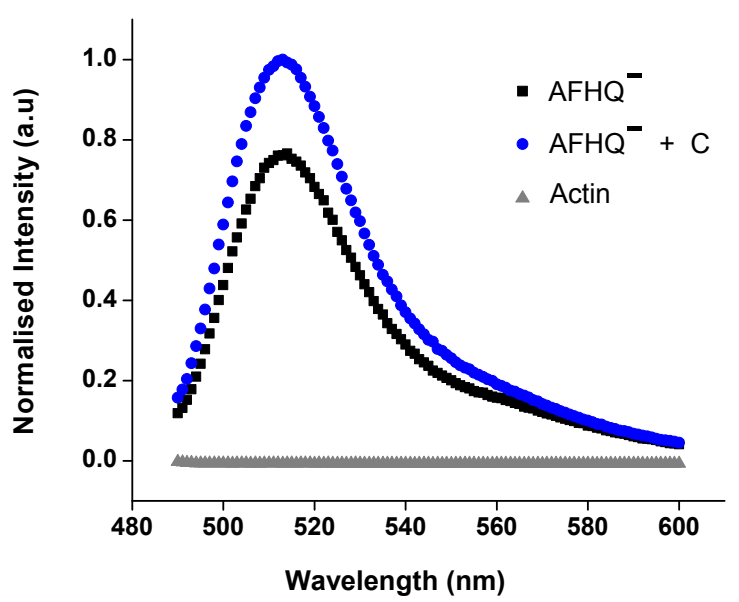

Figure 4.3.: Fluorescence of DNA sensors versus controls when crosslinked to actin. Emission spectra of DNA sensors and controls crosslinked to actin filaments. a) Working of DNA sensors in actin (0.5:1:1- F:H:Q). b) Working of controls in actin (0.5:1:1-F:H:Q). Gray line represents the intensity of uncrosslinked actin (entangled). F - Strand with fluorophore Alexa 488, H - Hairpin strand, Q - Strand with quencher molecule, C - Control strand. A - Actin. AFHQ: Quenched sensors in actin, AFHQC: Opened sensors in actin (opened by $C$ strand). $\mathrm{AFHQ}^{-}$: Closed controls in actin. $\mathrm{AFHQ}^{-} \mathrm{C}$ : Opened controls in actin (opened by $\mathrm{C}$ strand). The values plotted are averaged for $n=5$

curve in Fig. 4.3 ). Controls lack the quencher dye and are denoted as $\mathrm{Q}^{-}$. AFHQ $\mathrm{AFers}^{-}$ to controls in the closed state crosslinked to actin, while $\mathrm{AFHQ}^{-} \mathrm{C}$ refers to controls in the open state crosslinked to actin. When measured in a spectrometer at a strand ratio of $0.5: 1: 1$, controls show high fluorescence in both of their states i.e closed (black curve in Fig $4.3 \mathrm{~b}$ ) and open (blue curve in Fig $4.3 \mathrm{p}$ ). The fluorescence of DNA sensors and controls were thus quantified in their respective conformation while crosslinked in a network. These spectral emission measurements serve as a proof of principle for the bulk efficiency of our molecular DNA force sensors via their controls in actin networks.

\subsubsection{Confocal Laser Scan: Quenching and Fluorescence of Sensors across the Network}

Confocal laser scan microscopy was used to quantitatively image sensor fluorescence in networks. The quantitative fluorescence of actin-DNA sensor networks in their quenched and open states was determined for different sensor concentrations. Likewise, the fluorescence of controls in the closed and open state in networks was quantified. The ratio of DNA sensor concentration to actin concentration was varied and is given by $R=$ DNA sensor concentration/actin concentration which ranges from $R=0.005,0.01,0.02,0.1$ up to 

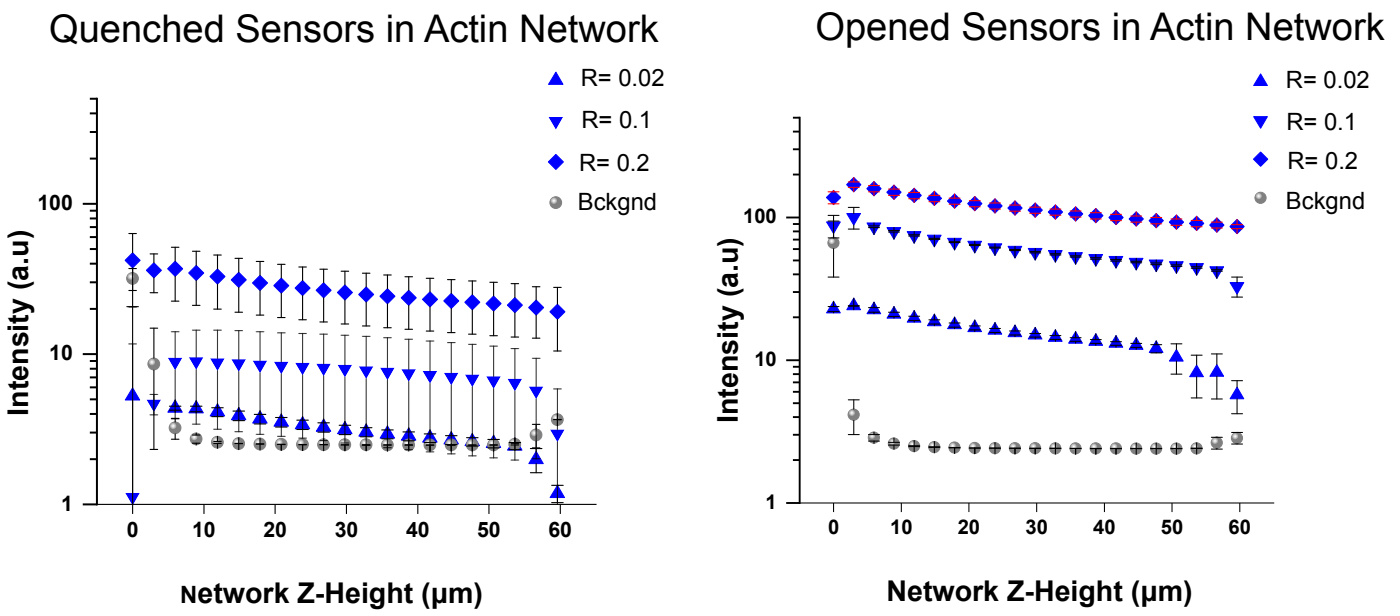

Figure 4.4.: Intensity of quenched and fluorescent sensors across actin network. A confocal z-stack image scan was made across the entire network's z-height at 10 different positions. Fluorescence of quenched (on the left) and opened sensors (on the right) in the network for different sensor concentrations (given by the $R$ value) are represented. DNA sensors remain quenched throughout the network and when opened also show a high fluorescence signal for all $z$-positions in the network at all probed concentrations. We do not represent the quenched and opened fluorescence for sensor concentrations of $R=0.005, R=0.01$ in networks, as they reach the detection limits of the detector and have high background fluorescence.

0.2. Z-stack imaging was done with a Leica microscope across the $60 \mu \mathrm{m}$ height of the network. The image acquisition settings are as described under section 2.2. These quantitative imaging experiments were done to choose an appropriate sensor concentration that gives a high fluorescence which can be used for FLIM measurements.

Figure 4.4 shows sensors fluorescence for concentrations $R=0.02, R=0.1 \& R=0.2$. In all of these concentrations, a uniformly quenched fluorescence is observed across the network's height (left side of Figure 4.4). Background denotes the uncrosslinked (entangled) actin network i.e. without any sensors crosslinked to actin. In the opened fluorescent state (right side of Fig. 4.4), sensors light up across the entire network, with an increased fluorescence high above the background signal. It is an order of magnitude higher than the quenched state of sensors. Networks with the sensor concentration $R=0.005$ and $R=$ 0.01 reach the detection limits of the detector. They do not show a signal notably different from the background signal and therefore are not shown in Fig. 4.4 Controls were also crosslinked to actin in the same manner as sensors and were quantified for their fluorescence in the closed and open state (Fig. 4.5). Their fluorescence intensity remains the same in the closed and opened state, although in the open state they are slightly brighter. From these confocal laser scan imaging, we quantitaively imaged the fluorescence of sensors in the actin-sensor networks, for their quenched and open state. This will help in the visualization of the presence of force chains ${ }^{\mathrm{i}}$ if present in networks.

\footnotetext{
${ }^{\mathrm{i} C h a i n ~ l i k e ~ r e g i o n s ~ o f ~ l a r g e ~ f o r c e s ~[20] ~}$
} 
Closed Controls in Actin Network

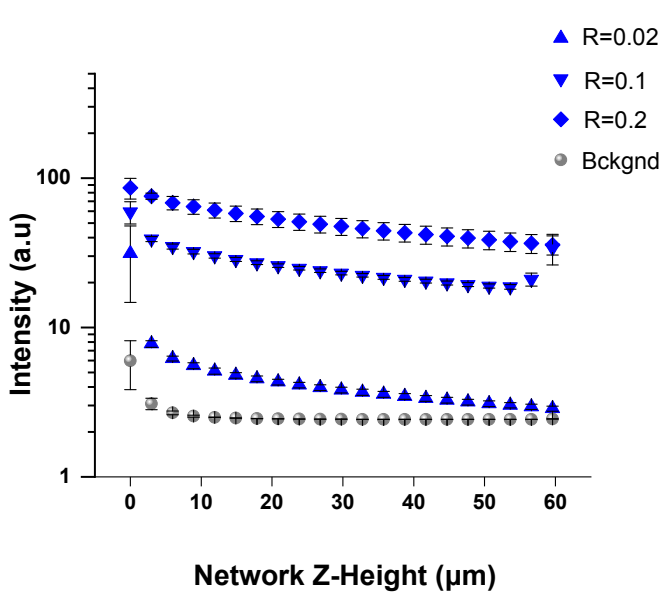

Opened Controls in Actin Network

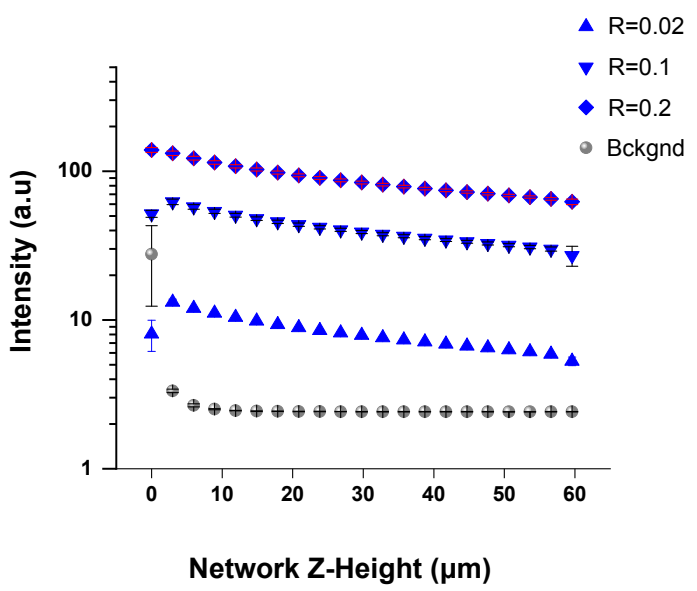

Figure 4.5.: Controls fluorescence intensity across actin network. The fluorescence of controls in the network for their closed and open states is depicted for different sensor concentrations (varying R). Their fluorescence in both states fairly remains the same. A z-stack image scan was made throughout the network $z$-height at 10 different positions. For $R=0.005, R=0.01$ networks, we do not show the fluorescence of the control as they also reach the detection limits of the detector.

\subsubsection{Fluorescence Lifetime Imaging Microscopy (FLIM) on Actin-DNA Sensor Network}

To characterize the DNA sensors quenching efficiency while in an actin network, we used the FLIM technique via the Time-Correlated Single Photon Counting (TCSPC). This was done at the lab of Jörg Enderlein, University of Göttingen. This key method allows sensitivity down to the single-molecule level. As described in Chapter 2, lifetime measurements are independent of the concentration of fluorophores or quencher molecules used. Hence we used this method to understand: what are the quenched lifetimes across the entire depth of the network? How many sensors remain quenched and do we have a homogeneous quenching throughout the entire network? What is the quenching efficiency across the network given the fact that the network itself may pose steric hindrances?

This section aims to answer these questions via FLIM in the actin-DNA sensor network at two different ratios of DNA sensor concentration to actin concentration ( $R=$ DNA sensor concentration / actin concentration. $R=0.01 \& R=0.1$ ). Throughout this thesis, a ratio of $R=0.01$ represents a sparsely crosslinked network and $R=0.1$ a densely crosslinked network. In both cases, actin concentration was kept at $1 \mathrm{mg} / \mathrm{ml}(23.81 \mu \mathrm{M})$. From Chapter 3, we had optimized the efficient quenching stoichiometry to be 0.5:1:1 - F:H:Q (i.e. half the molar concentration of $\mathrm{F}$ strands with respect to $\mathrm{H}$ and $\mathrm{Q}$ molar concentration). Thus, this stoichiometry is used in actin-sensor networks. FLIM measurements were then done 
in these networks for 4 conditions. (i) quenched sensors crosslinked to actin, (ii) opened sensors crosslinked to actin, (iii) closed controls and (iv) opened controls crosslinked to actin.

\subsubsection{FLIM on Sparsely Crosslinked Actin-DNA Sensor Network $(R=0.01)$}

Fluorescence lifetime imaging (FLIM) on in vitro reconstituted actin-DNA sensors network was performed on a home-built confocal setup equipped with a galvo-scanner (FLIM-Bee, Picoquant). The excitation unit is the same as described for experiments in solution. Measurements were done as follows: areas of $20 \mu \mathrm{m} \times 20 \mu \mathrm{m}$ on the sample surface were scanned with a frame rate of $5 \mathrm{~Hz}$ with the aid of the galvo-scanner. Recording of one frame was done at each z-plane with an interval of $1 \mu \mathrm{m}$ up to $5 \mu \mathrm{m}$ from the surface. Photons collected from each scanned area at each z plane was used to compute the TCSPC histogram for the respective plane. Following this, the tail of the histogram was fitted using the bi-exponential decay function ( $0.5 \mathrm{~ns}$ after the maximum) using the same maximum likelihood procedure. The fitting function in this case was

$$
I(t)=\frac{A_{1}}{\tau_{1}} e^{-t / \tau_{1}}+\frac{A_{2}}{\tau_{2}} e^{-t / \tau_{2}}+b
$$

where $A_{1}$ represents the amplitude of the lifetime $\tau_{1}, A_{2}$ the amplitude of $\tau_{2}$ and $\mathrm{b}$ is the background

\section{Quenched sensor-actin network}

The red curve in Fig. 4.6 represents the fluorescence decay for quenched sensors which has a bi-exponential component. Sensors that are quenched, decay faster with a short lifetime, which is seen as a slightly bent region in the curve in the first few nanoseconds (5 ns). It signifies the presence of a quenched lifetime (short lifetime) of DNA sensors. The remaining part of the red curve in Fig. 4.6 decays with long lifetime photons (from 5-25 ns). By fitting a bi-exponential function (Eqn 4.1 ) to the entire curve, we obtain two amplitudes $\left(A_{1} \& A_{2}\right)$ for their respective lifetimes. These amplitudes reflect the two different populations of quenched sensors in the network. The longer lifetime population which is around $75 \%-87 \%$ of the total sensor population in networks are sensors that do not quench. This might be because they either lack a $Q$ strand in their fully assembled state or are misassembled. Since they do not quench they may also not crosslink actin filaments as both $\mathrm{F}$ and $\mathrm{Q}$ are needed to quench as well as to crosslink. The quenched (shorter) lifetime component population is between 13\% - 25\% which indicates that $Q$ strands are present in the sensor in a manner available for quenching. We speculate that this small population may represent the actual amount of sensors that quench as well as crosslink actin filaments (via F and Q strands). Both, the short and longer lifetimes along with their population (amplitudes) for each z-height in the network are given in Table 4.1 


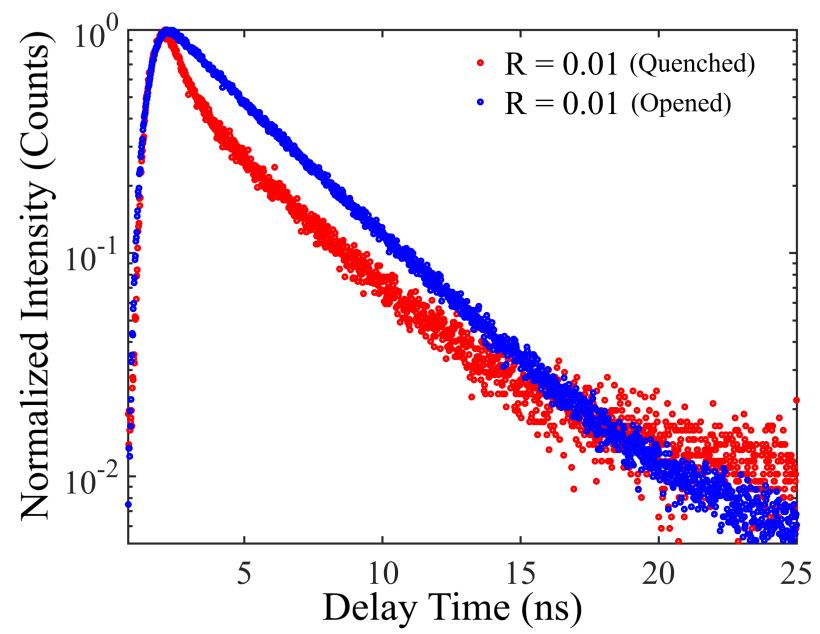

Figure 4.6.: Fluorescence lifetime decay curves of $R=0.01$ actin-DNA sensor network in 0.5:1:1 stoichiometry. The red curve shows the fluorescence lifetime decay of quenched sensors in the actin network. They have a bi-exponential decay where quenched photons that have shorter lifetimes, decay fast $(<5 \mathrm{~ns})$. This is seen as a small bent region in the upper part of the red curve. The remaining part of this curve decays with the longer lifetime photons ( $>$ $5 \mathrm{~ns}$ ). The blue curve displays the fluorescence decay of opened sensors in the actin network where all photons have a single lifetime (a straight line decay curve).

We speculate that the following reasons can lead to the existence of the longer lifetime in quenched sensors in the network. These longer lifetime sensors may exist in an unassembled manner either as free F strands or as partially assembled configurations where only the $\mathrm{F}$ and $\mathrm{H}$ strands might be present both of which lead to a long lifetime (non-quenched). A fully assembled sensor may also be present, but due to thermal fluctuations, the $F$ and $Q$ strands may not be available for quenching via FRET thus leading to a longer lifetime. We also performed an alternative analysis with the same data to see if the population of quenched sensors increases. To do this, we used the same bi-exponential fitting function to decay curves but fixed the longer lifetime as a constant parameter. By this analysis, we only observed that the quenched lifetime population did not increase but rather reduced to $8 \%-15 \%$ which is represented in Table 4.2 . The lifetime decay curves of quenched sensor-actin and opened sensor-actin network is shown in Fig. 4.6 
Table 4.1.: Quenched sensors lifetime and their amplitude (in percentages) in $R=0.01$ actin network at different z-positions

\begin{tabular}{lll}
\hline Network z-height $(\mu \mathrm{m})$ & $\tau_{1}(\mathrm{~ns})$ & $\tau_{2}(\mathrm{~ns})$ \\
\hline 0 & $0.77 \pm 0.04(13 \%)$ & $3.75 \pm 0.05(87 \%)$ \\
5 & $0.71 \pm 0.06(14 \%)$ & $3.68 \pm 0.06(86 \%)$ \\
10 & $0.73 \pm 0.07(18 \%)$ & $3.73 \pm 0.04(82 \%)$ \\
15 & $0.71 \pm 0.04(20 \%)$ & $3.80 \pm 0.08(80 \%)$ \\
20 & $0.63 \pm 0.05(21 \%)$ & $3.80 \pm 0.07(79 \%)$ \\
25 & $0.52 \pm 0.08(19 \%)$ & $3.56 \pm 0.08(81 \%)$ \\
30 & $0.63 \pm 0.07(25 \%)$ & $3.64 \pm 0.06(75 \%)$ \\
35 & $0.51 \pm 0.04(21 \%)$ & $3.73 \pm 0.04(79 \%)$ \\
40 & $0.54 \pm 0.06(23 \%)$ & $3.71 \pm 0.04(77 \%)$ \\
45 & $0.45 \pm 0.06(22 \%)$ & $3.86 \pm 0.04(78 \%)$ \\
\hline
\end{tabular}

Table 4.2.: Quenched sensors lifetime and amplitude (in percentages) in $R=0.01$ actin network when the longer lifetime $\left(\tau_{2}=3.5-3.70 \mathrm{~ns}\right)$ is held as a constant

\begin{tabular}{ll}
\hline Network z-height $(\mu \mathrm{m})$ & $\tau_{1}(\mathrm{~ns})$ \\
\hline 0 & $0.66 \pm 0.06(8 \%)$ \\
5 & $0.71 \pm 0.04(10 \%)$ \\
10 & $0.60 \pm 0.06(10 \%)$ \\
15 & $0.54 \pm 0.07(11 \%)$ \\
20 & $0.52 \pm 0.08(11 \%)$ \\
25 & $0.51 \pm 0.04(11 \%)$ \\
30 & $0.52 \pm 0.07(15 \%)$ \\
35 & $0.45 \pm 0.06(11 \%)$ \\
40 & $0.51 \pm 0.04(12 \%)$ \\
45 & $0.39 \pm 0.08(8 \%)$ \\
\hline
\end{tabular}

\section{Opened sensor-actin network}

Sensors opened by the $C$ (complementary) strand in the opened sensor-actin network were analyzed by fitting a mono-exponential function (Eqn 3.1). The blue curve in Fig. 4.6 represents the opened sensor network lifetime decay curve which indicates the existence of a longer lifetime. The mono-exponential fit function yielded a 100\% amplitude of photons with the longer lifetime of $3.6 \mathrm{~ns}$. A second short lifetime was not present. This shows that all sensors remain completely open when $C$ strand is added. The lifetime for opened sensors at various z-distances in the network are shown in Table 4.3 


\section{\begin{tabular}{l|l} 
Chapter 4 & DNA SENSORS IN IN VITRO ACTIN NETWORK \& IN CELLULAR ACTIN
\end{tabular}}

Table 4.3.: Opened sensors lifetime in $R=0.01$ actin network

\begin{tabular}{ll}
\hline Network z-height $(\mu \mathrm{m})$ & $\tau(\mathrm{ns})$ \\
\hline 0 & $3.66 \pm 0.06$ \\
5 & $3.66 \pm 0.07$ \\
10 & $3.69 \pm 0.05$ \\
15 & $3.84 \pm 0.04$ \\
20 & $3.61 \pm 0.04$ \\
25 & $3.73 \pm 0.04$ \\
30 & $3.71 \pm 0.06$ \\
35 & $3.68 \pm 0.07$ \\
40 & $3.68 \pm 0.08$ \\
45 & $3.71 \pm 0.04$ \\
\hline
\end{tabular}

\subsubsection{FLIM on Densely Crosslinked Actin-DNA Sensor Network $(R=0.1)$}

A densely crosslinked actin-DNA sensor network is represented as $R=0.1$ where the sensor concentration is tenfold higher compared to $R=0.01$. The optimized 0.5:1:1 (F:H:Q) stoichiometry was used here. FLIM was done in these quenched and opened sensor networks after an hour of polymerization. Care was taken to determine the lifetime of sensors in both of these networks exactly 1 hour after polymerization because this densely crosslinked network does not reach a steady-state of polymerization (explained in Chapter 5 in Fig. 5.1) rather it continues to form bundles post 1 hour of polymerization (Fig. 5.4).

\section{Quenched sensor-actin network}

Table 4.4.: Quenched sensor lifetime and amplitude (in percentages) in $R=0.1$ actin network

\begin{tabular}{lll}
\hline Network z-height $(\mu \mathrm{m})$ & $\tau_{1}(\mathrm{~ns})$ & $\tau_{2}=3.3-3.5(\mathrm{~ns})$ (a constant) \\
\hline 0 & $0.94 \pm 0.06(43 \%)$ & $3.52 \pm 0.06(57 \%)$ \\
1 & $0.98 \pm 0.04(40 \%)$ & $3.52 \pm 0.07(60 \%)$ \\
2 & $0.94 \pm 0.07(37 \%)$ & $3.35 \pm 0.08(63 \%)$ \\
3 & $0.92 \pm 0.06(35 \%)$ & $3.27 \pm 0.05(65 \%)$ \\
4 & $0.93 \pm 0.05(35 \%)$ & $3.20 \pm 0.06(65 \%)$ \\
5 & $0.90 \pm 0.08(33 \%)$ & $3.34 \pm 0.06(67 \%)$ \\
\hline
\end{tabular}

From the results of $R=0.01$ actin-sensor network, we had observed that lifetime remained the same all through the network's z-height $(50 \mu \mathrm{m})$. Therefore for this densely crosslinked $(R=0.1)$ network, lifetime measurements were performed only until a $\mathrm{z}$ height of $5 \mu \mathrm{m}$ into the network. Within this z-height of the network, we observed that quenched sensors populations are high, on an average of around $35 \%$ (Table 4.4 ) than 


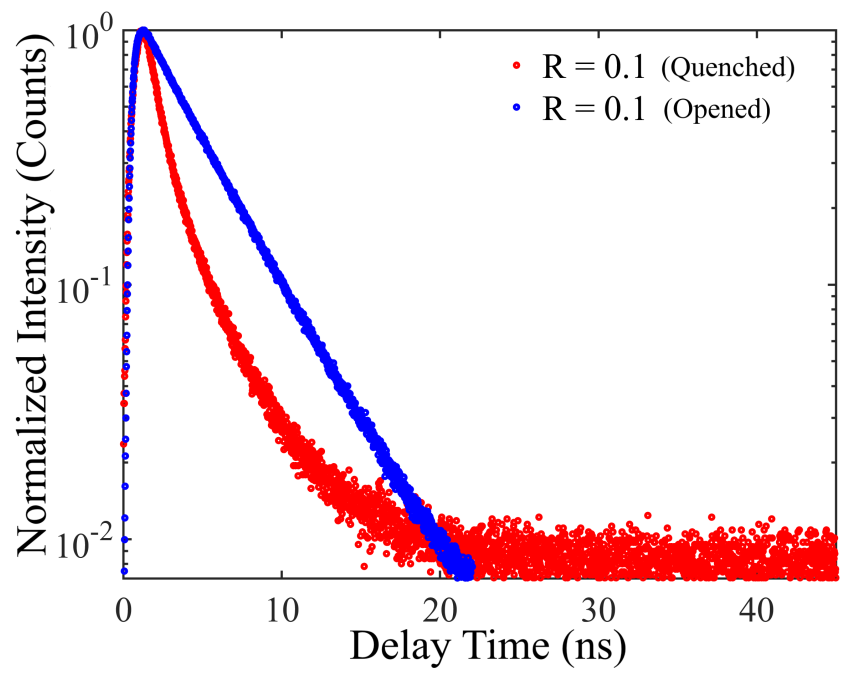

Figure 4.7.: Lifetime decay curves of $R=0.1$ actin-DNA sensor network in 0.5:1:1 (F:H:Q) stoichiometry. Red curve: Quenched sensors in actin network shows a well pronounced quenching. Observed as a bi-exponential decay curve, the bent region indicates shorter lifetime photons which further decays as longer lifetime photons. Blue curve: Opened sensors in actin network decay with a mono-exponential lifetime.

the sparsely crosslinked network which is $20 \%$ (see Table 4.1). Such a large population of quenched sensors represents an excellent quenching efficiency (more amount of sensors are quenched) in a crosslinked actin network which is the same quenching efficiency obtained for sensors in solution (DNA buffer - 35\%, see Chapter 3, Table 3.4. Possible reasons for such a large quenched population in this network might be due to the following. Sensors that quench may not be the ones crosslinked to the actin filaments. Rather, they may remain in the solution inside the network in a quenched configuration. This might lead to the observed large increase in the quenched population which was also seen in DNA buffer solutions. Concerning the lifetime, we observe a slightly higher value for the quenched lifetime ( $0.9 \mathrm{~ns})$ when compared to the sparsely crosslinked network, $R=0.01$ (0.5 - $0.9 \mathrm{~ns}$ ). The longer lifetime component which is $3.5 \mathrm{~ns}$ (Table 4.4 ) is also present in quenched sensors in this densely crosslinked network along with the short lifetime. This long lifetime could be either due to unassembled or partially assembled sensors. Also, due to the presence of bundles in this dense network (Fig. 5.4, sensors may attach along these bundles which can contribute to the observed increased quenching, without necessarily crosslinking the network in an isotropic manner. We reason that these may lead to the observed overall increase in the population of quenched sensors in this densely crosslinked network. Table 4.4 shows the lifetime of quenched sensors. 


\section{\begin{tabular}{l|l} 
Chapter 4 & DNA SENSORS IN IN VITRO ACTIN NETWORK \& IN CELLULAR ACTIN
\end{tabular}}

\section{Opened sensor-actin network}

Table 4.5.: Opened sensors lifetime in $R=0.1$ actin network

\begin{tabular}{ll}
\hline Network z-height $(\mu \mathrm{m})$ & $\tau(\mathrm{ns})$ \\
\hline 0 & $3.87 \pm 0.05$ \\
1 & $3.85 \pm 0.07$ \\
2 & $3.89 \pm 0.06$ \\
3 & $3.89 \pm 0.08$ \\
4 & $3.88 \pm 0.04$ \\
5 & $3.89 \pm 0.07$ \\
\hline
\end{tabular}

When the complementary (C) strand is used, we observe an opening of all sensors which is noticeable from the single lifetime in Tab. 4.5 and from the lifetime decay curve (without any bent region or a bi-exponent component) in Fig. 4.7 Since we use excess C strand $(20 \mu \mathrm{M})$, all the sensors in the network are completely opened thus leading to 100 $\%$ opened population.

\section{Controls crosslinked to actin $(R=0.1)$ at 0.5:1:1 stoichiometry}

Controls for our DNA sensors, as introduced before (Fig. 3.2 are constructs that have the same conformational structure as DNA sensors, but lack the quencher molecule in the $\mathrm{Q}$ strand. We determined the lifetime of controls via FLIM measurements while they were crosslinked to the actin filaments forming a network. $\mathrm{AFHQ}^{-}$denotes controls in the closed state crosslinked to actin filaments. $\mathrm{AFHQ}^{-} \mathrm{C}$ signifies controls in their open state crosslinked to actin. Characterization of these controls for their lifetimes in their closed and open state is a verification of the FRET-based quenching of DNA sensor. In these FLIM measurements for controls, we observe only non-quenched lifetimes for their closed and open state. Additionally, these measurements can also reveal the presence of any structural quenching, from the closed state controls, where then its lifetime then will be lower than opened state controls.

Table 4.6.: Controls lifetime in $R=0.1$ actin network for their closed state

\begin{tabular}{ll}
\hline Network z-height $(\mu \mathrm{m})$ & $\tau(\mathrm{ns})$ \\
\hline 0 & $3.28 \pm 0.05$ \\
1 & $3.40 \pm 0.07$ \\
2 & $3.48 \pm 0.06$ \\
3 & $3.53 \pm 0.08$ \\
4 & $3.58 \pm 0.04$ \\
5 & $3.59 \pm 0.07$ \\
\hline
\end{tabular}




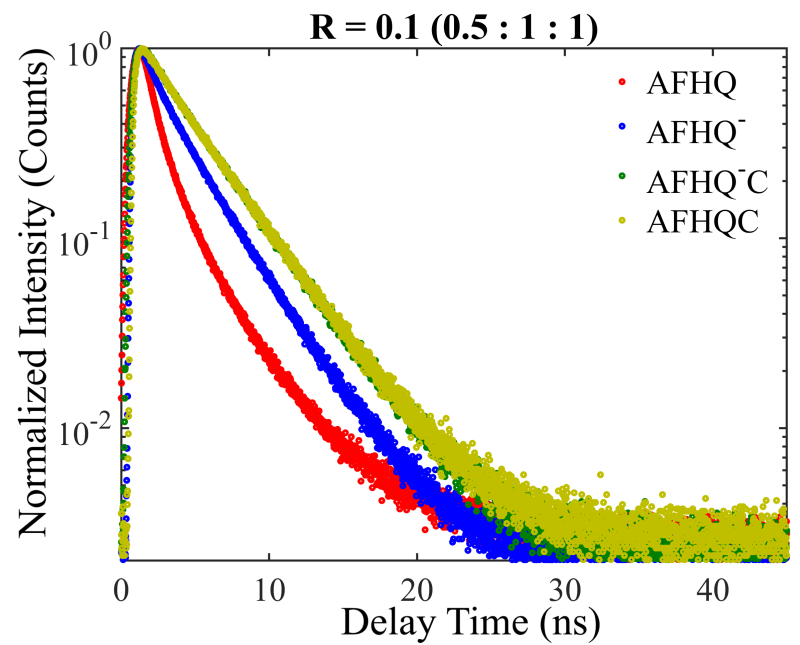

Figure 4.8.: DNA sensor and controls lifetime decay curves for $\boldsymbol{R}=\mathbf{0 . 1}$ actin-DNA sensor network. Quenched DNA sensors (AFHQ) decay with a bi-exponential component (red curve). When sensors are opened by $\mathrm{C}$ strand (AFHQC), a mono-exponential fluorescence decay (yellow curve) is observed. Controls both in the closed (blue curve, $\mathrm{AFHQ}^{-}$) and open (green curve below the yellow, $\mathrm{AFHQ}^{-} \mathrm{C}$ ) state decay mono-exponentially.

Table 4.7.: Controls lifetime in $R=0.1$ actin network for their opened state

\begin{tabular}{ll}
\hline Network z-height $(\mu \mathrm{m})$ & $\tau(\mathrm{ns})$ \\
\hline 0 & $3.72 \pm 0.07$ \\
1 & $3.90 \pm 0.04$ \\
2 & $3.94 \pm 0.06$ \\
3 & $3.95 \pm 0.05$ \\
4 & $3.95 \pm 0.05$ \\
5 & $3.95 \pm 0.07$ \\
\hline
\end{tabular}

From a mono-exponential fit function, we observe that the controls have a non-quenched longer lifetime of $3.5 \mathrm{~ns}$ in the closed state and around $3.9 \mathrm{~ns}$ in the open state across the actin network. We also observe a reduced lifetime in the closed state controls which is around $1.5 \mathrm{~ns}$. Since the amplitude (population) of this lifetime is less than $10 \%$ we consider it as negligible. Therefore we fix this as a constant parameter in the fitting of the lifetime decay curves. This small population of $1.5 \mathrm{~ns}$ may represent the noise of the system. Controls lifetime for each z-height in the network up until $5 \mu \mathrm{m}$ is given in Table 4.6 \& Table 4.7 and shown in Fig. 4.8

In summary, the characterization of DNA force sensors in actin networks at two different crosslinking densities, sparse $(R=0.01)$ and dense $(R=0.1)$, showed that sensors 


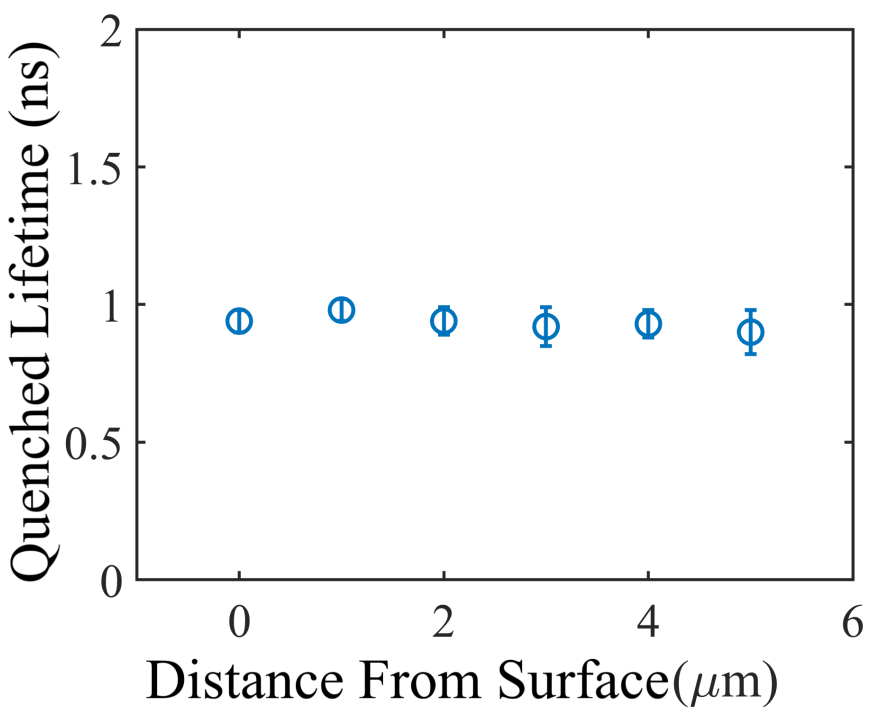

Figure 4.9.: Homogenous quenching of sensors in $\boldsymbol{R}=\mathbf{0 . 1}$ actin-DNA sensor network at 0.5:1:1 (F:H:Q) stoichiometry. A homogenously quenched lifetime is observed in the quenched sensor-actin network throughout the $5 \mu \mathrm{m}$ z-height.

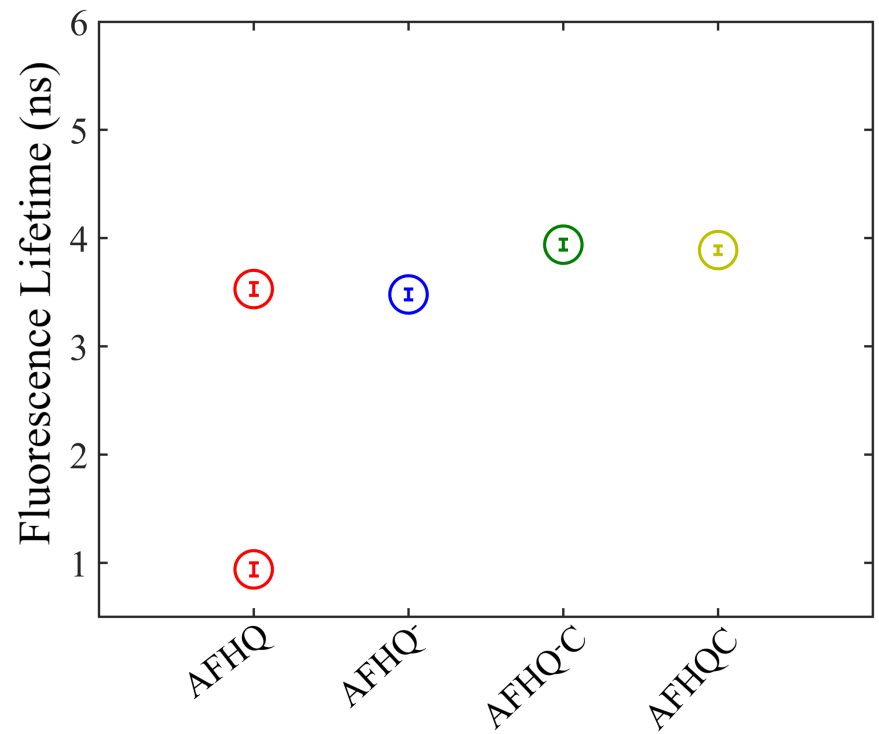

Figure 4.10.: Lifetimes of sensor and controls in $R=0.1$ actin-DNA sensor network for 0.5:1:1 (F:H:Q) stoichiometry. The lifetime of quenched and opened sensors in the actin-DNA sensor network and the lifetime of closed and opened controls in the actin-controls network are represented. AFHQ: Quenched sensor, $\mathrm{AFHQ}^{-}$: Closed controls, $\mathrm{AFHQ}^{-} \mathrm{C}$ : Opened controls, AFHQC: Opened Sensor. 
are homogeneously quenched in the network (Fig. 4.9. Table 4.1 and Table 4.4. This gives knowledge about the spatial distribution of sensors across the actin network. Since the quenching is homogeneous throughout the entire network $(50 \mu \mathrm{m})$ in $\mathrm{R}=0.01$ network, we measured the quenched lifetime for $R=0.1$ network up until only $5 \mu \mathrm{m}$ which is shown in Fig. 4.9. The summary of sensor lifetimes (AFHQ \& AFHQC) and control lifetimes ( $\mathrm{AFHQ}^{-} \& \mathrm{AFHQ}^{-} \mathrm{C}$ ) for the densely crosslinked network, $R=0.1$ are given in Fig. 4.10 Quenched sensors (AFHQ) have two lifetimes (quenched and long) given by red circles in Fig. 4.10. The opened sensor (yellow circle) and controls (blue \& green circles) show as expected, longer lifetimes for their fluorescence states. On an extra note, the longer lifetime for the quenched sensor (upper red circle, AFHQ) in Fig. 4.10 has the same lifetime as the closed controls $\mathrm{AFHQ}^{-}$(blue circle). This may suggest that these longer lifetime quenched sensors in the network are probably without the quencher strand.

\subsection{DNA Sensors in Fibroblast's Actin}

The aim of developing our force sensor is to also measure force distributions across cytoskeletal networks inside the cell. As described in chapter 11. polymeric actin that constitutes the cellular cortex is responsible for transmitting internal forces [55]. To understand force distributions across these networks, we chose to attach DNA sensors to F-actin inside cells. The attachment of sensors to cellular F-actin follows the same attachment strategy as for in vitro networks, the RFP lifeact - haloTag and haloligand as described in Fig. 4.1.

HaloTag is genetically cloned with RFP lifeact. This plasmid is first introduced into cells as a first nucleotransfection (4D Nucleofector, Core Unit (AAF-1002B), X-Unit (AAF - 1002X), Lonza, Switzerland) [2]. Once they were stably expressed after transfection (around 24 hours), the second transfection was done. To do this, haloligand modified DNA sensors were assembled in vitro and transfected through a viafect transfection medium. The detailed method of transfection is described in A.2.2. 3T3 Fibroblast cells (ACC 173) were used for these experiments. FLIM was performed on these cells $36 \mathrm{hrs}$ after the first lifeact transfection.

\subsubsection{DNA Sensors in 3T3 Fibroblasts}

We performed FLIM measurements on live 3T3 fibroblasts in $\mathrm{CO}_{2}$ independent medium (Gibco, 1X, 18045-05, Germany) to test the functioning of sensors using the same confocal setup as for actin networks. Scans were recorded on multiple areas identifying one cell for each area. Lifetime scans were done at frame rate of $0.1 \mathrm{~Hz}$. For measurements on cells, TCSPC histogram of each pixel was fitted using a bi-exponential decay function and the intensity weighted average lifetime was computed for each pixel. The intensity weighted average lifetime is the false colour scale in the FLIM images presented here. The imaging duration lasted for 20 minutes. Fluorescence lifetimes were monitored for 6 minutes after 
a)
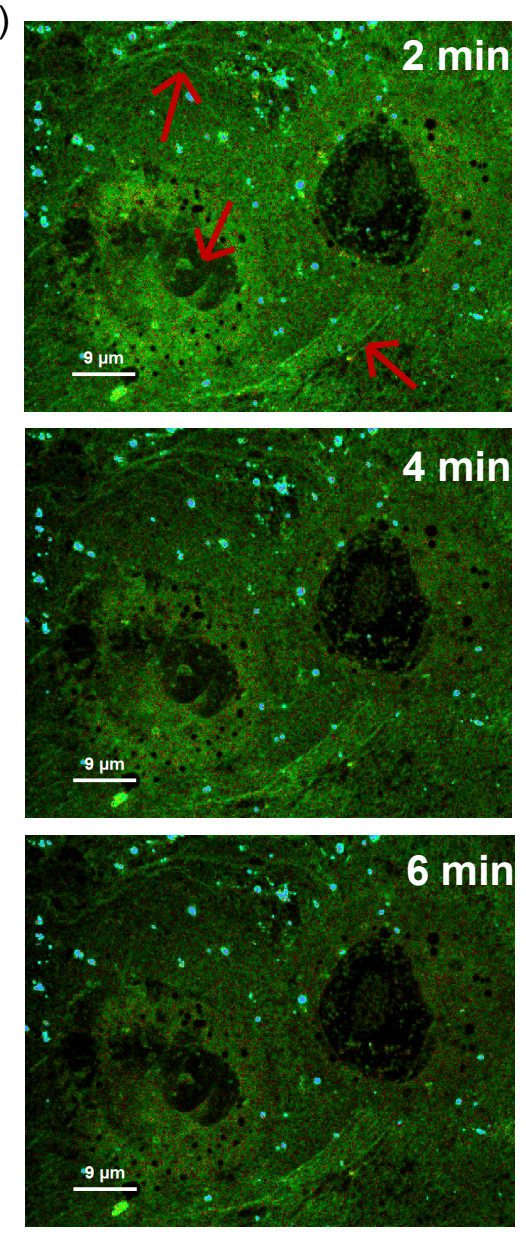

b)

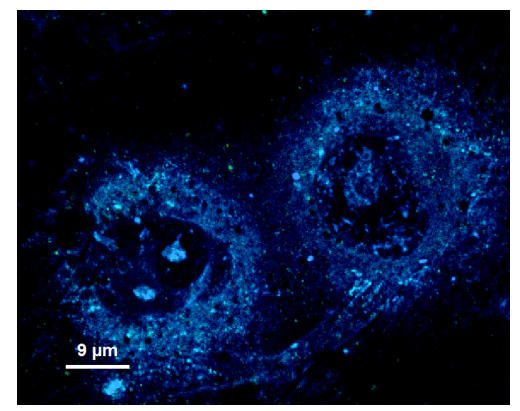

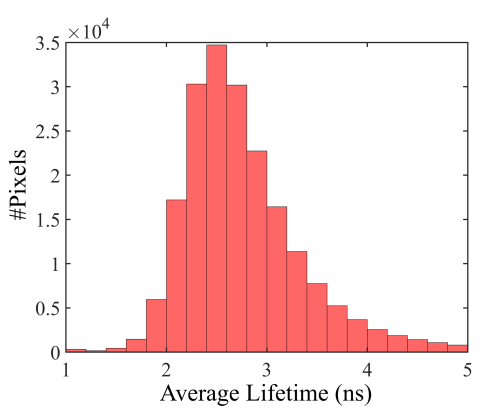
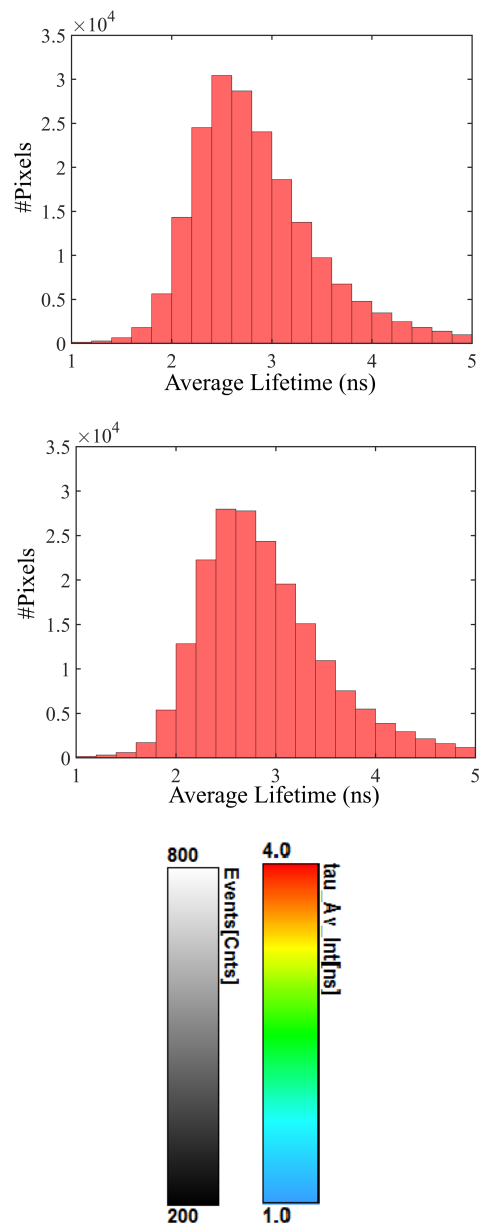

Figure 4.11.: DNA sensors inside live 3T3 fibroblasts. Two $3 T 3$ fibroblasts clustered next to each other are imaged via FLIM. Lifetimes are reported for frames between 0 and 2 min as 2 $\min$, frames between 2 and $4 \mathrm{~min}$ as $4 \mathrm{~min}$, frames between 4 and $6 \mathrm{~min}$ as $6 \mathrm{~min}$. a) represents sensor channel with excitation wavelength as $488 \mathrm{~nm}$. b) RFP channel where actin structures are visualized through the fluorescence of RFP Lifeact. Arrows indicate the presence of actin structures visualized in the sensor channel. The mean lifetimes of sensors are $2.7 \pm 0.5 \mathrm{~ns}$. 
the start of the experiment. We do not take into account any experimental data beyond 6 minutes since fluorescence signal dropped significantly due to photobleaching. The data is represented for frames between 0 and 2 minutes as $2 \mathrm{~min}$, frames between 2 and $4 \mathrm{~min}$ utes as 4 minutes and frames between 4 and 6 minutes as 6 minutes.

Figure 4.11 represents the sensor channel (excitation wavelength - $488 \mathrm{~nm}$ ) \& Fig. $4.11 \mathrm{p}$ represents the actin channel (RFP lifeact). Two fibroblasts that are clustered next to each other were imaged. The dark black structure is the nucleus. Sensors are absent inside the nucleus, but are seen in the nucleolus and as a thin arc-like structure (indicated by red arrows) across the nucleus. Sensors' absence in the nucleus is also confirmed in the actin channel (Fig. 4.11p - RFP lifeact image which stains actin structures) where the entire nucleus is seen as a dark black structure. The distribution of intensity weighted average lifetime of the sensor for each pixel of Fig. 4.11 was done. The lifetime of sensors inside cells is found to be $2.7 \pm 0.5 \mathrm{~ns}$ (mean and width of lifetime distribution) in the first 2 minutes and remains fairly unaltered till 6 minutes after the start of the experiment.

We observe that our DNA sensors localize to stress fibers (actin structures) which is seen from the sensor channel (Fig. 4.11 ), indicated by red arrows. They are visible at the top left corner and the bottom right corner of the $2 \mathrm{~min}$ image (Fig. 4.11a). The bottom right corner stress fiber is visible in both, the sensor and the actin channel. This validates that the DNA sensors localize on actin structures and are not degraded by the cell until the time of FLIM imaging ( $6 \mathrm{~h}$ after 'DNA sensor transfection'). The top left corner stress fiber (indicated by the red arrow) is seen only in the sensor channel but not visible in the actin channel. Ideally, this stress fiber should also be observed in the actin channel (RFP image, Fig. 4.11p). This would imply sensor localization in actin structures. However, this is not the case in the present context. This might be due to the reason that FLIM imaging of cells was done after the peak expression of RFP in cells (i.e. the timepoint of the highest fluorescence signal had elapsed when the FLIM imaging was done). Hence the fluorescence was faint or not visible for the mentioned stress fiber. In general, the presence of actin structures (stress fiber) both in the sensor and actin channel are a good preliminary indication of DNA sensor localization to actin structures (stress fiber). Another observance is the granular appearance throughout the whole cytoplasm (entire space) of the cell in the sensor channel. This might be due to the excess concentration of sensors, which may be localized into vesicles that fills the cytoplasmic space. 


\section{\begin{tabular}{l|l} 
Chapter 4 & DNA SENSORS IN IN VITRO ACTIN NETWORK \& IN CELLULAR ACTIN
\end{tabular}}

\subsubsection{Controls in 3T3 Fibroblasts}

Next, we performed FLIM measurements on "controls" that were also transfected into live 3T3 fibroblasts. The transfection protocol that was followed was the same as DNA sensors A.2.2). Fig. 4.12 represents controls channel (excitation wavelength - $488 \mathrm{~nm}$ ) and Fig. $4.12 \mathrm{p}$ the actin channel (RFP lifeact). Compared to DNA sensors, we observe that the mean of the distribution of average lifetimes for controls per pixel is shifted to $3.5 \mathrm{~ns}$ with a width of $0.8 \mathrm{~ns}$. The total distribution of controls lifetimes has two distinct peak regions as seen in Fig. 4.12 . The left shoulder region in the peak is the shorter lifetime which comes from the region between the two cells in Fig. $4.12 \mathrm{a}$ (seen as green in false-color scale). It may be due to local heterogeneities inside the cell that modulate the controls lifetime. Such a modulated lifetime is also observed in the RFP lifetime (actin channel - Fig. 4.12 ) of controls, where in this region, RFP also has a lifetime (from the false-color scale) different from other regions of the cell. This validates that microenvironmental changes in this region lead to the varying lifetimes of controls. Thus, further experiments are needed to account for such complexities. If one takes into account only the distribution towards longer lifetimes i.e. 3-5 ns, the mean value shifts towards $\sim 4 \mathrm{~ns}$. Similar trend is observed for frames between 2 and 4 minutes and 4 and 6 minutes. This longer lifetime distribution from controls (no FRET) in cells as against the DNA sensors (short lifetime) shows a promising direction of our DNA sensors function in a live cellular environment. Lifetimes above $4 \mathrm{~ns}$ are due to the low number of photons in certain pixels and due to background. Moreover, we observe that controls also co-localize to actin which is well noticed from the fluorescence of the actin channel (Fig. 4.12 $\mathrm{a}$ ) that is the same as the controls channel. This is because RFP lifeact binds to actin. The dark black region in control and actin channel is the nucleus where we do not find any presence of controls.

It is known that in experiments involving live-cell FRET, other complications persist such as cellular auto-fluorescence, the modulation of fluorophore properties due to environmental conditions of $\mathrm{pH}$, ion concentration, temperature, photobleaching effects etc [28]. Given these complications, we find a difference in lifetime exists between DNA sensors $(2.7 \pm 0.5 \mathrm{~ns})$ and controls $(\sim 4 \mathrm{~ns})$ (Fig. 4.13). We also observed that DNA sensors remain in fibroblasts up to 6 hours (timed from the second step of transfection) and that DNA sensors and controls both colocalize to some actin structures. Our results from FLIM on DNA sensors in cells represent a preliminary proof that our sensors function inside live fibroblasts. Further experiments and design of new controls will be required in the future for a detailed characterization of the sensor in a cellular environment. 

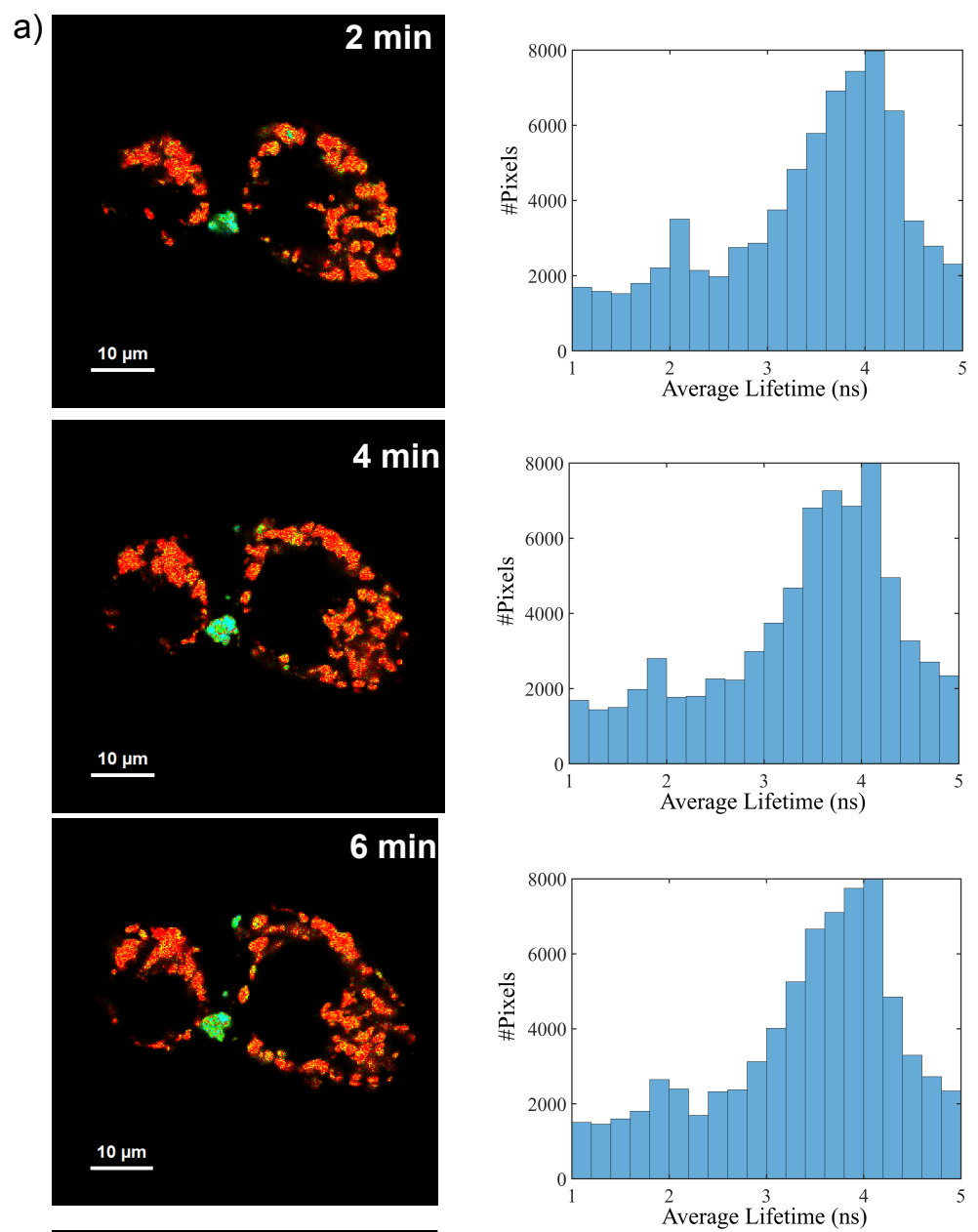

b)
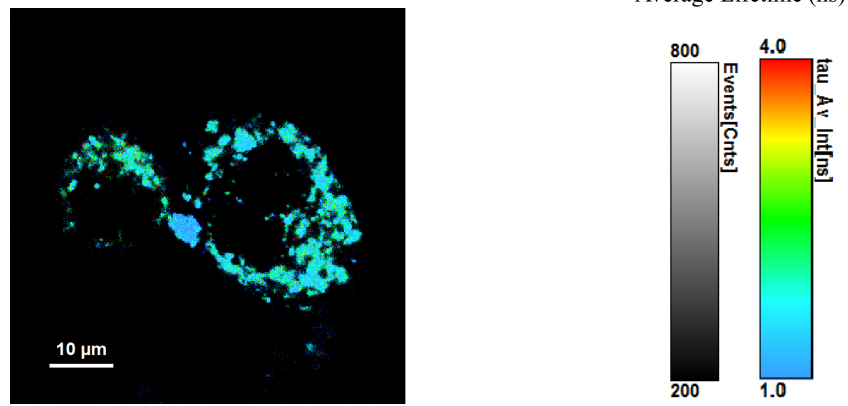

Figure 4.12.: Controls inside adherent 3T3 fibroblasts. a) Controls inside 3 T3 fibroblasts and their respective lifetimes until 6 minutes of the imaging duration at $488 \mathrm{~nm}$ wavelength. b) The RFP lifeact channel of controls for fibroblasts which validates that controls bind to actin structures via lifeact. The mean lifetime of controls in fibroblast is around $3.5 \mathrm{~ns}$, lifetimes between $0-2 \mathrm{~ns}$ in the histogram originate from the small region connecting the two cells. Lifetimes above 4.0 ns represent noise. Frames between 0 and $2 \mathrm{~min}$ are represented as $2 \mathrm{~min}$, likewise between 2 and $4 \min$ as $4 \mathrm{~min}, 4$ and $6 \min$ as $6 \min$. 


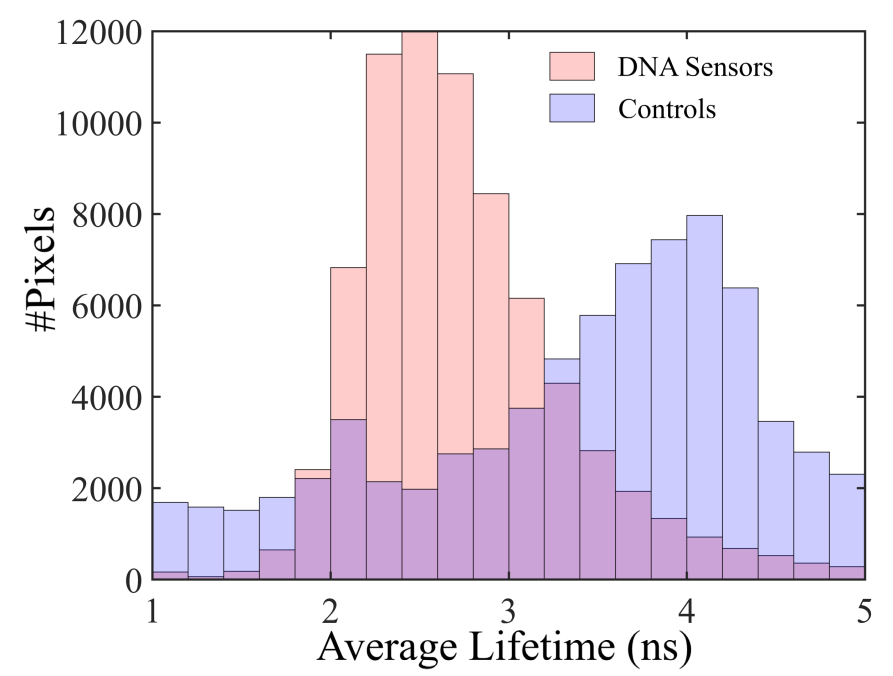

Figure 4.13.: The lifetime of DNA sensors versus controls in 3T3 fibroblasts. The mean lifetime of DNA sensors is shorter which is around $2.7 \pm 0.5 \mathrm{~ns}$ and controls lifetime are longer around $\sim 4$ ns in live fibroblasts. Lifetimes above 4 ns represent changes in the environment.

\subsubsection{Discussion}

With the spectrometer, we quantified the bulk fluorescence of our FRET DNA sensors while it was attached to actin filaments. From confocal laser scanning imaging experiments, we find that sensors at 0.5:1:1 stoichiometry quench and fluoresce for varying sensors concentrations $(R)$ in the network. Through this quantitative imaging, we also observed that they are distributed throughout the network. We also proved the functionality of sensors by quantifying controls non-quenched fluorescence across these networks through the same confocal laser scans. Characterization of sensors while crosslinked to actin was the first and foremost priority. This was achieved through FLIM measurements in this chapter. Two networks, sparsely crosslinked $(R=0.01)$ and densely crosslinked $(R=$ 0.1 ) were probed. From these experiments we find that our sensors quench uniformly across the network i.e. they undergo FRET throughout the whole network.

The quenching efficiency in the sparsely crosslinked network $(R=0.01)$ is $22 \%$ and gets decreased to $11 \%$ (when the longer lifetime component is fixed as a constant in the analysis). In the densely crosslinked network $(R=0.1)$, we have an excellently quenched population (36\%) similar to the quenched population in solution (36\% in DNA buffer, Chapter 3 , Table 3.2). Although the quenching efficiency is high in networks, at present we do not know, if this increased quenched population arises from sensors present in the solution of the network (i.e those that are not crosslinked to actin filaments) or if they are contributed from sensors that are crosslinked to actin filaments. To understand this, the results from $R=0.01$ network and the following control experiments might explain. The reduced pop- 
ulation of quenched sensors $(11 \%)$ observed in the $R=0.01$ network may, in fact, be the actual population of sensors that remain crosslinked to actin and quench at the same time without the influence of thermal fluctuations or steric hindrances from the network.

The two sets of control experiments that can be done are as follows. The first is where an actin network with uncrosslinked sensors $(R=0.1)$ can be used, to determine the population of sensors that are quenched but not crosslinked to actin i.e those that remain in the solution of the network. If this population is closer to the increased quenched sensor population (36\%) observed for the $R=0.1$ network population, it signifies that this increase arises from the quenched but uncrosslinked sensors (ones that remain in solution). The second control experiment is to employ sensors with only $\mathrm{F}$ and $\mathrm{H}$ strands in the actin-sensor network, and perform a FLIM. This will yield a population of non-quenched sensors with a long lifetime (since $Q$ strand is absent), that are crosslinked but do not quench. This can then be compared to see if they match with the population of long lifetime quenched sensors in $R=0.1$ network, which are also crosslinked but do not quench. By these two experiments, we can then determine the actual population of sensors that quench and are crosslinked to actin to account for the increase in the amount of quenched sensors in $R=0.1$ network.

To sum up, on sensors in cells, we find that DNA sensors and controls show a noteworthy difference inside cells. This is evident from the difference in the lifetime between the DNA sensors and controls given in Fig. 4.13 Since this is our first attempt to test DNA sensors in live cells, we do not conclude any existence of distribution of forces inside the cell, but rather we present here our first result of a working hypothesis in a highly complex cellular environment. We also highlight the evidence of attachment of our molecular DNA force sensors to certain actin structures.

It is observed that lifeact cannot bind to filopodia of mesenchymal cells [108] or nuclear actin rods [92]. When forces across such actin structures are to be probed, DNA sensors can be tuned at its attachment site. Actin binding proteins (ABPs) other than lifeact or actin nanobodies can be employed at the attachment end. Many other available actin binding tools [82] can simply be incorporated into our DNA sensors via the haloTag fusion protein technology. In this way, DNA sensors are pre-assembled in vitro and then introduced into cells to measure force distribution across a desired cytoskeletal structure by the two-step transfection protocol that was followed in this work.

Multiple control experiments are needed when determining the lifetime of the sensor inside the cell. Positive and negative control tests, that can be performed are a measurement of cellular autofluorescence, measurement of the lifetime of a cell without sensors, and introduction of sensors into cells without attaching them to actin. To isolate the local environmental influence, sensors can be introduced into fibroblasts that are fixed (dead 
and membrane permeabilized) before making a FLIM measurement. DNA sensors need to be also checked for nuclease degradation. Furthermore, a timeline-based live cell FLIM imaging needs to be done, which would help us gain insight on how long DNA sensors remain within the cell before being degraded.

Other interesting investigations that can be carried out are by attaching mutant ABPs on our sensors which will help understand different stress relaxation mechanisms and force distributions due to their delayed unbinding rate from actin. An example of such a mutant ABP is the ACTN4-K255E, which strongly binds to actin due to exposure of a third cryptic actin binding site [134], [135]. In conclusion, we have utilized the FLIM method for the characterization of sensors in actin networks and for probing their working inside cells. Also, by incorporating various sets of FRET pairs for different lengths of hairpins in our sensors, we can fine-tune and develop sensors to probe multiple force ranges simultaneously. 


\section{Mechanics of Actin-DNA Sensor Networks}

\subsection{Introduction}

Cells exhibit solid- and fluid-like material properties at specific timescales, i.e. they are viscoelastic [22], [16]. This is due to the transient nature of cytoskeletal networks and active mechanics. These networks dissolve and reform on timescales defined by the unbinding rate of actin-binding proteins (ABPs). To understand such viscoelastic behavior, a study of mechanical stress inside the cell is needed. Since the cellular environment is highly complex, a reductionist bottom-up approach is generally chosen where model networks are reconstituted in vitro by utilizing the cytoskeletal components. Actin, in particular, is largely used as it can be easily crosslinked into in vitro networks by employing the same ABPs the cell utilizes.

In this chapter, we investigate the bulk stiffness, time-dependent viscoelastic behavior and the morphology of our model actin-DNA sensor networks. To do these we use macrorheology, microrheology, and confocal imaging technique. We used Lifeact, an actinbinding peptide, to crosslink our DNA sensors to actin filaments. In this way, we could tune the mechanical properties of our model networks by varying the DNA sensor concentration. Crosslinking DNA sensors to actin networks also facilitate the feasibility of studying stress relaxation in these composite model networks due to the transient nature of Lifeact which unbinds from actin at $0.4 \mathrm{~s}$ [106]. Therefore with our DNA sensors, we 


\section{\begin{tabular}{l|l} 
Chapter 5 & MECHANICS OF ACTIN-DNA SENSOR NETWORKS
\end{tabular}}

hope to later study force distributions in these networks spatiotemporally. By having a combined approach such as studying the mechanics of the network from macrorheology alongside the FRET- FLIM imaging (described in Chapter 4) of sensors for force distributions in the network, and with future studies on stress relaxation (from macrorheology), we aimed to understand the failure mechanisms beyond the non-linear response.

\section{Experimental Procedure}

Actin and actin-sensor networks were prepared by adding polymerization buffer to double distilled water, followed by the addition of G-actin. The solution was mixed gently to avoid the breakage of filaments while pipetting [55]. For imaging purposes, Atto $647 \mathrm{~N}$ Phalloidin was immediately added to the sample mixture. It was then pipetted on to a microscopic slide (631-1550, VWR, Germany) and covered with a coverslip (No.1.5, $24 \times 24 \mathrm{~mm}, \mathrm{VWR}$, Germany). Edges of the coverslip were sealed with VALAP The sample was then left undisturbed at room temperature for 1 hour to allow for network polymerization. For macro and microrheology experiments the preparation of actin and actin-DNA sensor networks were the same as above except that Atto 647N Phalloidin was not added. In a macrorheology experiment, the sample solution mixture after preparation was immediately added onto the rheometer plate and the experiment was started. For microrheology, the same steps of sample preparation were followed except that $1 \mu \mathrm{m}$ silica particles were added to the double-distilled water and sonicated for 5 minutes before the addition of polymerization buffer.

The preparation of the actin-DNA sensor network is as follows. Polymerization buffer was diluted in double distilled water, soon after which G-actin was added. To this mixture, RFP-lifeact-haloTag (expressed as a protein) was added. Pre-assembled DNA sensors were then added which have their F strand (fluorophore strand) and $Q$ strand (quencher strand) chemically modified on their outer ends to incorporate a haloligand. The haloligand covalently binds to haloTag thereby attaching it to actin filaments (elaborated in Fig. A.1. Atto $647 \mathrm{~N}$ Phalloidin was then added at the end in the case of imaging experiments.

\subsection{Macrorheology of Actin and Actin-DNA Sensor Networks}

Due to the semi-flexible nature of actin ${ }^{\text {ii }}$, it forms stiff networks at both low and high crosslinker concentrations [73],[116],[40],[15],[39]. Such semiflexibility also renders the networks with a time-dependent elastic and viscous behavior which is quantified by the shear modulus that scales with frequency $(\omega)$ as $\omega^{3 / 4}$ [74],[44],[111],[91],[41],[59]. Our model networks consist of rigid rods of actin and DNA force sensors as flexible linkers.

\footnotetext{
i VALAP is a sealant containing vaseline, lanolin, and paraffin - preparation is described in A.1

${ }^{i i}$ With their persistence length $\left(l_{p}=17 \mu \mathrm{m}\right.$ orders of magnitude larger than their filament diameter $=7 \mathrm{~nm}$ [42]), filaments resist bending when thermal forces act but exhibit small fluctuations.
} 
With DNA's persistence length on the order of $50 \mathrm{~nm}\left(l_{p} \sim \mathrm{nm}\right)$ and actin's persistence length on the order of $10 \mu \mathrm{m}\left(1_{p} \sim \mu \mathrm{m}\right)$, actin is considered as a rigid rod as its much stiffer than DNA. A network comprising both can then be considered as an effective medium where elastic properties are determined from those of the rods and linkers. The networks compliance is governed by flexible linkers (DNA sensors) where stiffer actin filaments act as a scaffold for the flexible crosslinkers thereby ensuring a network rigidity.

In a macroscopic shear deformation, we investigate the compliance of these networks as the elastic shear modulus $\left(G^{\prime}\right)$. The different sensor concentrations are given by the ratio $R=$ crosslinker concentration (DNA sensors)/actin concentration. The crosslinking ratios and their corresponding molar concentration are given in Table 5.1. Hence $R=0$ indicates a purely entangled actin network. By varying the sensor concentration, crosslinked actin-DNA sensor networks with different mechanical stiffness were produced. The concentration of actin in all networks remains as $23.81 \mu \mathrm{M}(1 \mathrm{mg} / \mathrm{ml})$.

Table 5.1.: Networks and crosslinking ratios

\begin{tabular}{l|c|c}
\hline Network & $\boldsymbol{R}$ & Molar Concentration $(\mu \mathbf{M})$ \\
\hline Actin & 0 & 23.81 \\
\hline Actin - DNA Sensor & 0.005 & 0.12 \\
& 0.01 & 0.24 \\
& 0.02 & 0.48 \\
& 0.1 & 2.4 \\
& 0.2 & 4.7 \\
\hline
\end{tabular}

\subsubsection{Gelation Kinetics: Elastic Shear Modulus of Networks}

The gelation kinetics, the frequency response of purely entangled actin network, and crosslinked actin-DNA sensor networks were monitored at $23{ }^{\circ} \mathrm{C}$ with a $25 \mathrm{~mm}$ cone and plate geometry at an opening angle of $1^{\circ}$ in a strain-controlled rheometer (MCR 501, Anton Paar). To overcome evaporation effects proper hydration of samples was ensured (by placing wet paper towels around the measuring plates) during the measurement.

Crosslinking the DNA sensors to actin filaments increased the network elasticity over time which is observed as an increase in the elastic shear modulus $\left(G^{\prime}\right)$ when compared to an entangled actin network's elasticity. The polymerization of actin into an entangled network takes place by the elongation of filaments which is evident from the rising $\mathrm{G}^{\prime}$ value over time. The entangled network reaches a steady-state, which is observed as a plateau in $\mathrm{G}^{\prime}$ of $0.4 \mathrm{~Pa}$ at $1 \mathrm{~Hz}$ and $1 \%$ strain amplitude. The increase in $\mathrm{G}^{\prime}$ for actin-DNA sensor networks indicates elongation and crosslinking of actin filaments into networks by 


\section{Actin-DNA Sensor Networks}
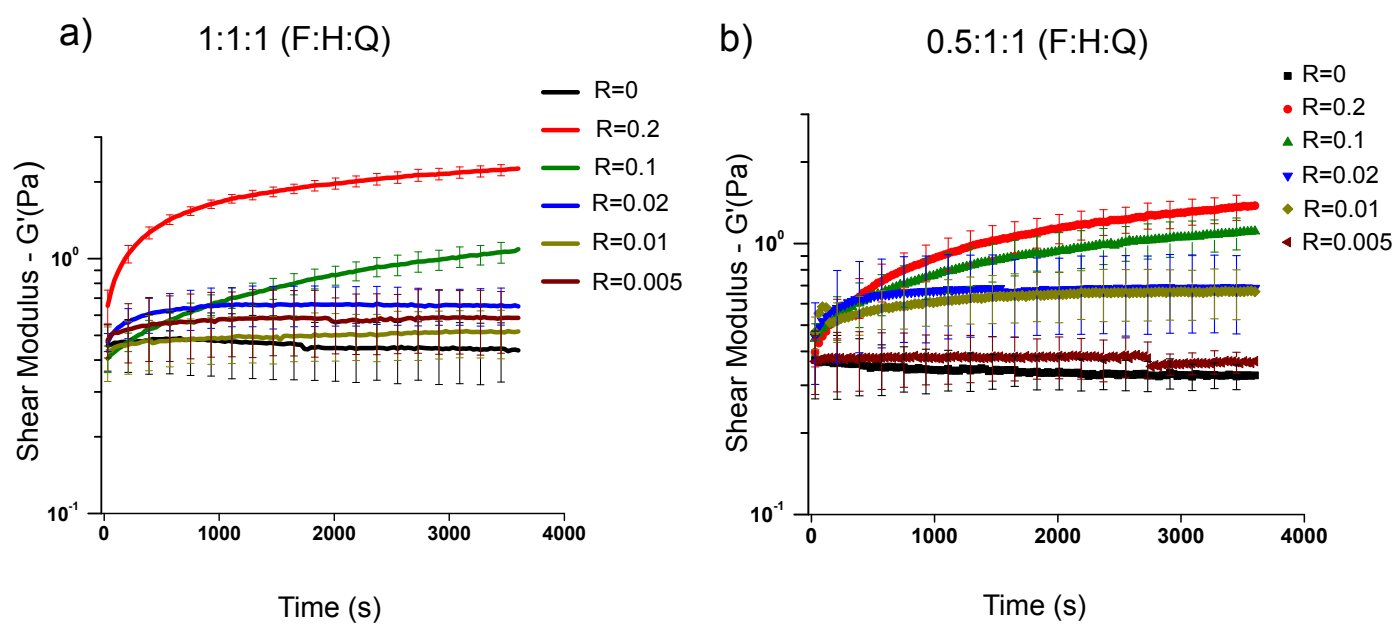

Figure 5.1.: Gelation kinetics of actin and actin-DNA sensor networks. The polymerization of actin and actin-DNA sensor networks are monitored in a rheometer as a time sweep experiment at $1 \mathrm{~Hz}$ and $1 \%$ osicllatory shear deformation. a) Equal stoichiometry (1:1:1 - F:H:Q) networks. b) Half stoichiometry $(0.5: 1: 1-\mathrm{F}: \mathrm{H}: \mathrm{Q})$. The different sensor concentration given by the ratio $R$ sensor concentration/actin concentration. $R=0.005$ (left open triangle), $R=0.01$ (diamond), $R=0.02$ (bottom inverted triangle), $R=0.1$ (upper triangle), $R=0.2$ (circle). $R=$ 0 in gray represents a purely entangled actin network (uncrosslinked) at $23.81 \mu \mathrm{M}(1 \mathrm{mg} / \mathrm{ml})$ molar concentration. Data represents mean \pm S.D of $n=3$.

the sensors which are seen from the varying stiffnesses $\left(G^{\prime}\right)$ of the networks (Fig. 5.1). We investigated elasticity of crosslinked networks for both of the F:H:Q ratios, the 1:1:1 and 0.5:1:1.

In the most densely crosslinked state (highest sensor concentration, $R=0.2$ ), we observe a notable six-fold increase in $G^{\prime}$ from the purely entangled actin network for both the ratio networks. While a steady state is reached for most of the actin-sensor networks within 1 hour of polymerization, densely crosslinked networks $(R=0.1$ and $R=0.2)$ has an increasing $G^{\prime}$ even after 1 hour of polymerization. These networks do not exhibit an elastic plateau after 1 hour of polymerization but continue their crosslinking further, which can be seen as an increasing $G^{\prime}$ (Fig. 5.1. This increasing $G^{\prime}$ due to the high number of crosslinks in the network retards the glassy dynamics of the network which would otherwise push the network towards equilibrium (steady-state).

Overall, we observe a stiffening of actin-DNA sensor networks (increased G') that depicts the effect of a physically cross-linked network of actin filaments and DNA sensor crosslinkers. Mechanical signatures of a six-fold increase and an unreached steady-state for higher DNA crosslinker concentration has been previously reported for a similarly crosslinked actin-DNA networks [69]. 
5.2.2 Bulk Frequency Response of Networks

\section{Actin - DNA Sensor Networks}

a)

$1: 1: 1(\mathrm{~F}: \mathrm{H}: \mathrm{Q})$

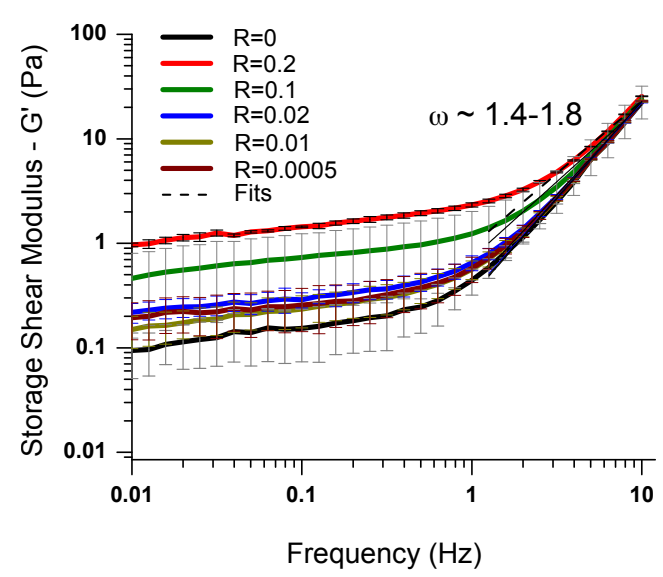

c)

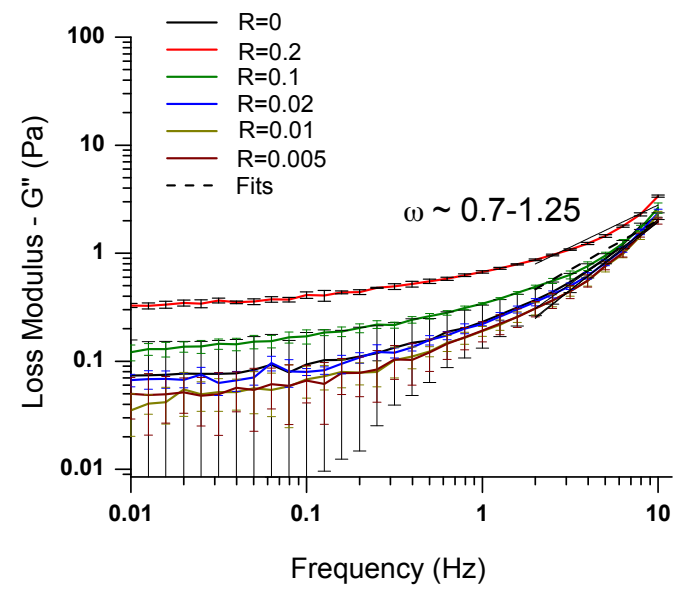

b)

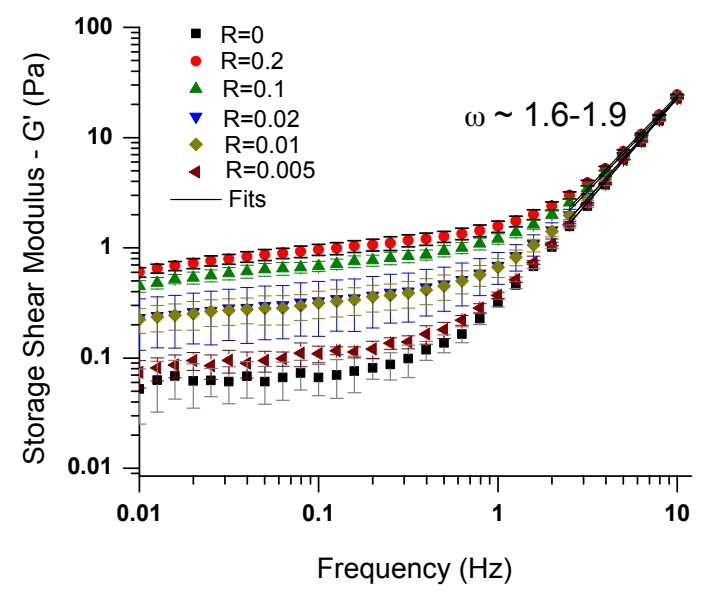

d)

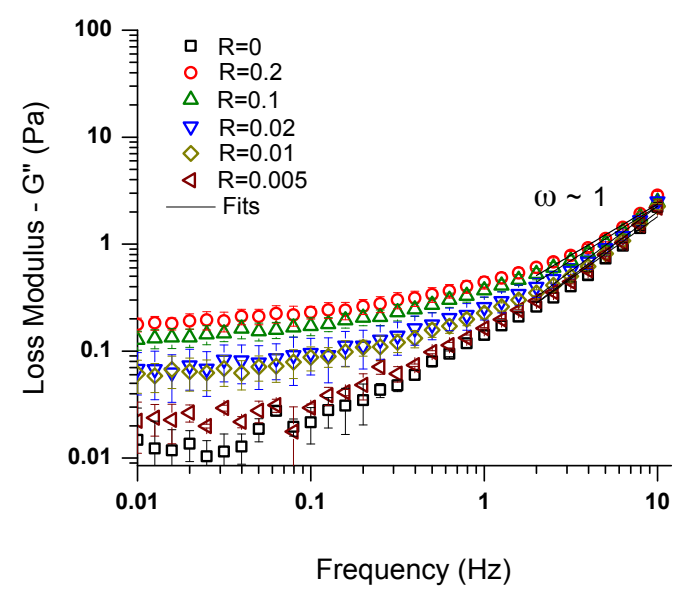

Figure 5.2.: Bulk frequency response of actin, actin-sensor networks in a shear deformation. The plots represent the storage modulus $\left(G^{\prime}\right)$ of a) 1:1:1, b) 0.5:1:1 networks and the viscous loss modulus $\left(G^{\prime \prime}\right)$ of c) 1:1:1, d) 0.5:1:1 networks in an oscillatory shear deformation at $1 \%$ strain in a macrorheometer. The $G^{\prime}$ is weakly dependent on the frequency with an elastic plateau at low frequencies and a power-law scaling regime at high frequencies. G' of networks are lower in magnitude than $\mathrm{G}^{\prime}$. It has a power-law scaling between 1.4-1.9. Actin concentration $=23.81 \mu \mathrm{M}(R=0)$. The different sensor concentrations given by the ratio $\mathrm{R}$, are $R=0.005$, $R=0.01, R=0.02, R=0.1$ and $R=0.2$. Their respective molar concentrations are between $0.12 \mu \mathrm{M}$ upto $4.7 \mu \mathrm{M}$. Data shows a mean of $n=3 \pm$ S.D. 


\section{\begin{tabular}{l|l} 
Chapter 5 & MECHANICS OF ACTIN-DNA SENSOR NETWORKS
\end{tabular}}

The frequency response of transiently crosslinked networks is dictated by actin entanglements and biochemical properties of the crosslinker e.g. the crosslinker unbinding, the binding strength $(\Delta \mathrm{E})$ or transition state position of crosslinker. We in this work, have varied only one parameter, the crosslinker unbinding which will influence the frequency response of actin-DNA sensor networks by exhibiting elastic and viscous behavior at specific time scales. The unbinding of our DNA sensors from actin filaments arises due to lifeact unbinding from actin filaments at $0.4 \mathrm{~s}$ [106]. Thus, we investigate the viscoelastic frequency behavior of actin-DNA sensor networks in a frequency sweep experiment in the linear small deformation limit (1\% amplitude of strain).

From the frequency spectrum in Fig. 5.2. the response of the networks can be distinctly divided into two regimes. An elastic plateau regime that is observed at low frequencies and a power-law scaling regime at high frequencies. There is no discernible elasticity observed at high frequencies, in which all of the different sensor concentrations behave the same (i.e. $\mathrm{G}^{\prime}$ converge on the same line). In the low-frequency regime $(0.01-1 \mathrm{~Hz})$, the elastic plateau $\left(G^{\prime}\right)$ of the network increases as the sensor concentration is increased. This indicates the characteristic solid-like elastic behavior of networks at low frequencies for all the given sensor concentrations.

Actin-DNA sensor networks do not exhibit any specific frequency dependent change in their viscoelasticity. Yet they are viscoelastic, meaning, their elastic modulus ( $\left.G^{\prime}\right)$ is always an order of magnitude higher than their viscous loss modulus ( $\left.G^{\prime \prime}\right)$ across the probed frequency range (Fig. 5.2). Their viscoelastic responses follow a behavior similar to entangled actin networks. There is only a marginal difference in the viscoelastic properties between the both, except that those crosslinked networks are more elastic (increased $\left.G^{\prime}\right)$ than $G^{\prime}$ of the entangled actin.

Between the different sensor concentrations, there are no distinctive features observed concerning frequency in the linear network regime (Fig. 5.2). This suggests that there is no time scale-dependent behavior in the experimentally accessible frequency range. We also do not see any viscous dissipation (a local minimum in $\mathrm{G}^{\prime \prime}$ ) in the networks although the networks are transiently connected. Above $1 \mathrm{~Hz}$, all networks show a powerlaw dependent response, where storage modulus scales with frequency as $G^{\prime} \sim \omega^{1.4-1.8}$ for 1:1:1 network and as $G^{\prime} \sim \omega^{1.6-1.9}$ for 0.5:1:1 network. The viscous loss moduli of networks scales as $G^{\prime \prime} \sim \omega^{0.7-1.25}$ for $1: 1: 1$ and $G^{\prime \prime} \sim \omega$ for $0.5: 1: 1$ networks. This frequency dependent power-law scaling of our networks at high frequencies is similar to an actinpalladin network where $\mathrm{G}^{\prime} \sim \omega^{2}$ and $\mathrm{G}^{\prime \prime} \sim \omega$ [47]. 


\subsection{Imaging of Actin-DNA Sensor Networks Morphology}

\section{Equal Stochiometry Networks (1:1:1 - F:H:Q)}

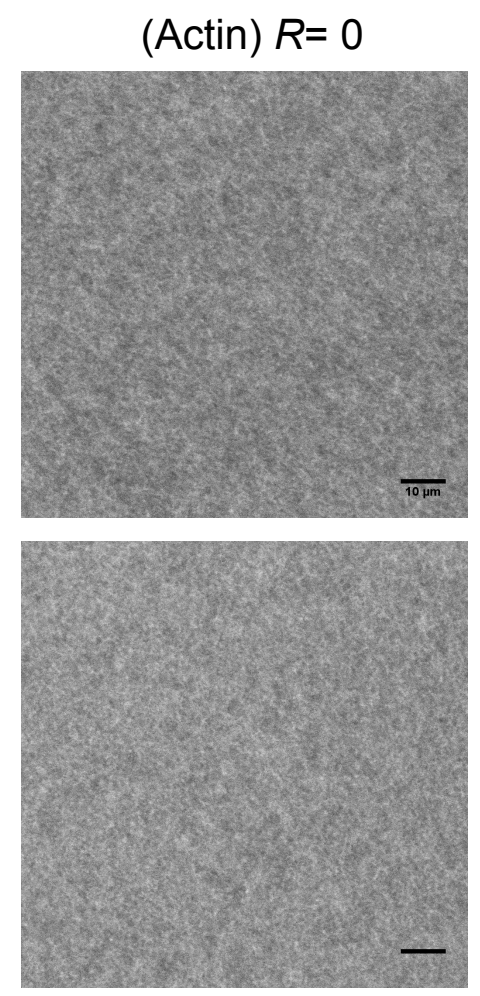

$R=0.02$
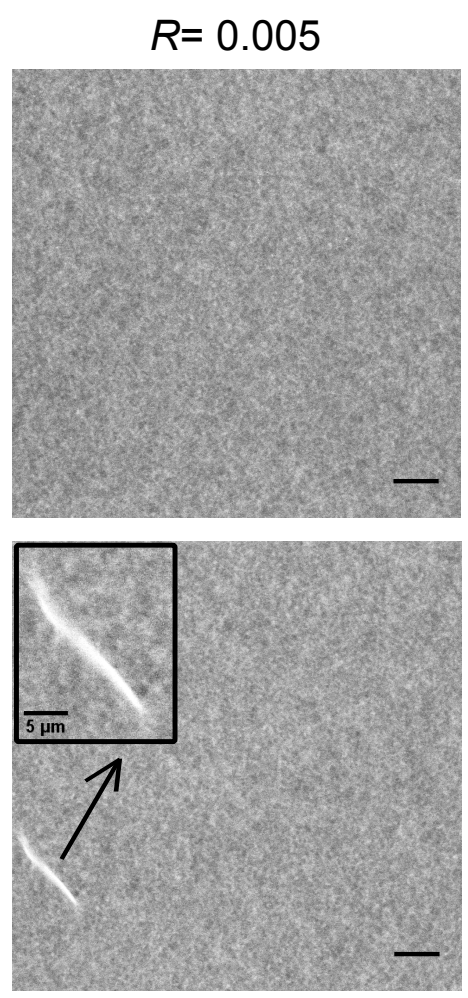

$R=0.1$
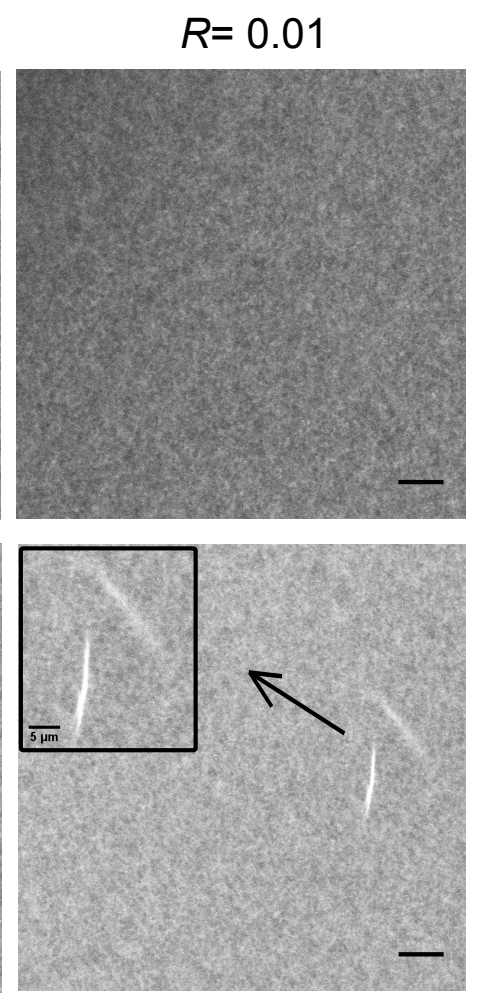

$R=0.2$

Figure 5.3.: Microstructure of equal stoichiometry (1:1:1) actin-sensor networks. Confocal laser scan images of actin-DNA sensor networks with equal stoichiometry of strands $F, H, Q$ in the sensor. At high crosslinking densities $(R=0.1, R=0.2)$ composite networks develop in which bundles are seen to appear along with an isotropically crosslinked network. At low crosslinker density $(R=0.005, R=0.01, R=0.02)$ the network microstructure is similar to an entangled actin $(R=0)$. Inset and arrows indicate the presence of bundles. Scale bar of micrographs: 10 $\mu \mathrm{m}$. Scale bar of inset: $5 \mu \mathrm{m}$

Confocal laser scan microscopy was used to qualitatively image the actin-DNA sensors network morphology. We correlate these network microstructures to their elastic stiffness which was determined by the elastic shear modulus $G^{\prime}$ from macrorheology experiments. Morphology of actin-DNA sensor network remains unaltered at low crosslink densities $(R=0.005, R=0.01, R=0.02)$, but changes to different structural morphologies at high crosslink ratios $(R=0.1, R=0.2)$. We used a laser scanning confocal microscope (SP5, Leica, Germany) to image the network heterogeneities by fluorescence staining the actin 


\section{Half Stochiometry Networks (0.5:1:1 - F:H:Q)}
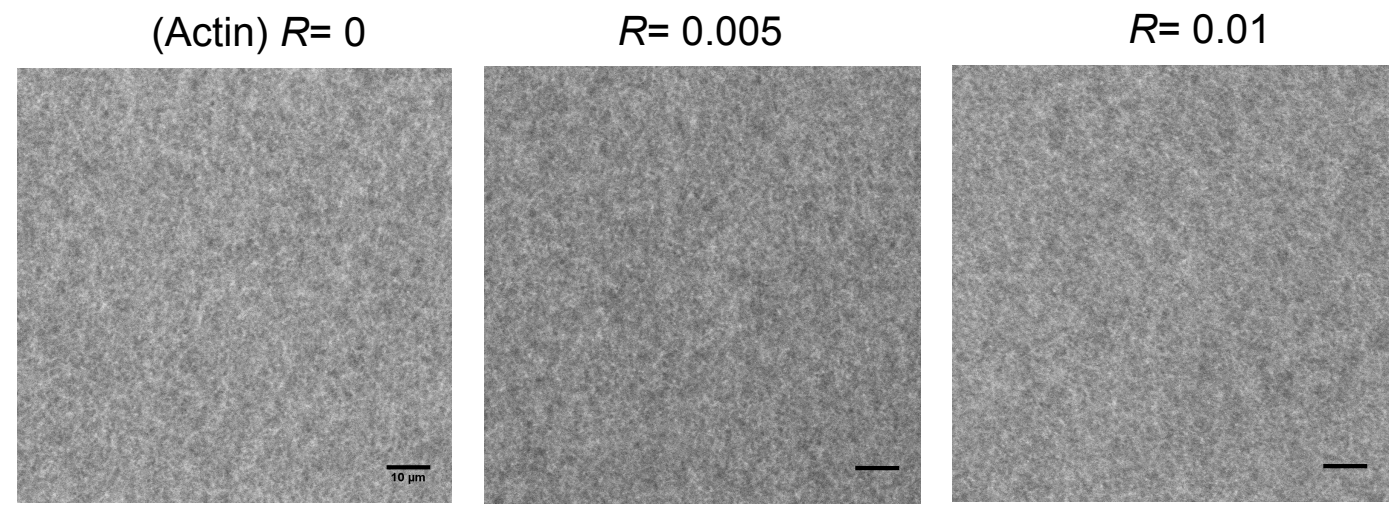

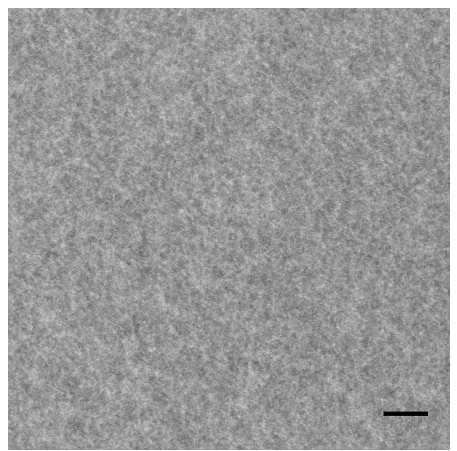

$R=0.02$

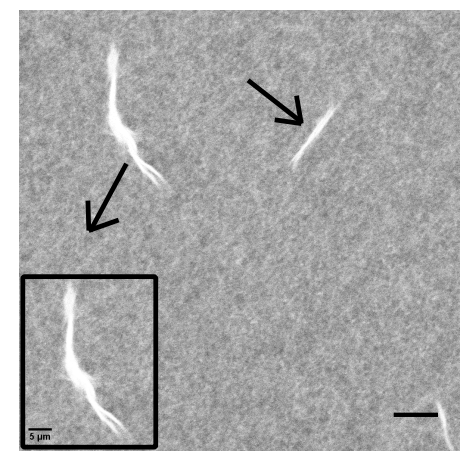

$R=0.1$

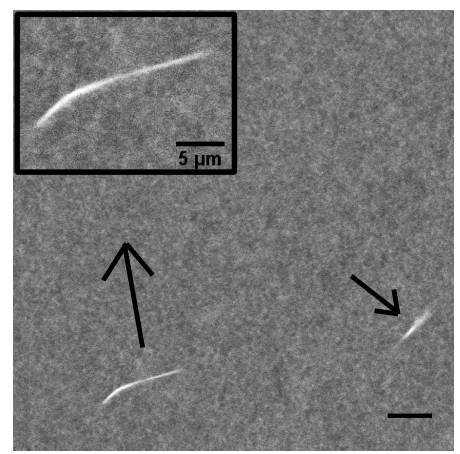

$R=0.2$

Figure 5.4.: Microstructure of half stoichiometry (0.5:1:1) of actin-sensor networks. Confocal laser scan imaging of actin sensor networks with half $F$ strands in the sensor with respect to $\mathrm{H}$ and $\mathrm{Q}$ strands (0.5:1:1 - $\mathrm{F}: \mathrm{H}: \mathrm{Q})$. Isotropic microstructure for $R=0.005, R=0.01$, $R=0.02$ and a composite network structure with the presence of bundles are observed at higher sensor concentrations $(R=0.1, R=0.2)$. Arrows indicate the presence of bundles in the network. Inset: Shows the zoom of the bundle in focus. Scale bar of micrographs: $10 \mu \mathrm{m}$. Scale bar of inset: $5 \mu \mathrm{m}$

with Phalloidin 647N (Atto Tec GmbH, Germany). Networks were imaged an hour after polymerization as described in sec 2.2

In equal stoichiometry networks (1:1:1 - F:H:Q) at low crosslink ratios, networks are isotropically crosslinked $(R=0.005, R=0.01, R=0.02 \mathrm{in} \mathrm{Fig.} \mathrm{5.3).} \mathrm{Visually} \mathrm{they} \mathrm{appear} \mathrm{the}$ same as that of entangled actin $(R=0$ in Fig. 5.3. However, at a threshold concentration $(R=0.1)$ a structural transition in the network is observed, where they have a composite structure. In this composite phase, short bundles appear embedded in an isotropic crosslinked scaffold $(R=0.1, R=0.2$ in Fig. 5.3). Bundles are fewer in number and do not dominate the microstructure after 1 hour of polymerization. 
In the half stoichiometry networks (0.5:1:1 - F:H:Q), the networks microstructure is morphologically the same as that of equal stoichiometry networks (1:1:1) for all crosslinking ratios. At low sensor concentrations $(R=0.005 R=0.01, R=0.02$ in Fig. 5.4), networks are isotropically crosslinked which are visibly indistinguishable from an entangled actin ( $R=0$ in Fig. 5.4). However, at high crosslink ratios (for $R=0.1$ and $R=0.2$ in Fig. 5.4), we observe the presence of bundles embedded in the crosslinked scaffold, similar to 1:1:1 stoichiometry networks (Fig. 5.4).

\subsection{Optical Trap: Microrheology of Actin and Actin-DNA Sensor Net- work}

Microrheology of actin and actin-DNA sensor networks was done by single-particle microrheology (1PMR). In order to choose an optimal laser power for these experiments, we recorded the bead displacement in water and actin $(1 \mathrm{mg} / \mathrm{ml})$ for different laser power settings between $8 \mathrm{~mW}$ to $113 \mathrm{~mW}$. The quoted laser powers are the ones measured before the laser enters the objective. Our observations are similar to what has been previously reported in [11]. We see the same trend of the shift in corner frequency $\left(f_{c}\right)$ as a function of wavelength (upper image in Fig. 5.5.

\subsubsection{Laser Power Optimization}

In Fig. 5.5 the upper graph shows the power spectral density (PSD) for water. At lower frequencies, the effect of an optical trap on the particle is dominant which is visible as a plateau for all the different laser powers. For higher frequencies, the power spectra of all laser powers converge on a single line. This represents the free diffusion of the particle due to Brownian motion where the spectrum decays as $1 / f^{2}$. Free diffusion shows that the drag forces on the particle are larger than the trapping force. Alongside the shift in $f_{c}$ (indicated by the arrow in Fig. 5.5), the area under the PSD curve also decreases as laser power increases. This indicates that the particle is tightly trapped due to the increase in the trap stiffness.

The lower graph in Fig. 5.5 shows the PSD of a $1 \mathrm{mg} / \mathrm{ml}$ actin at different laser powers. As described in [11], the power spectrum for viscoelastic solutions is not a Lorentzian function as in Eqn 2.24 and in Fig. 2.7. which is also observed in our actin PSD data. At higher frequencies, the PSD of all laser powers coincides on one line as in water. For viscoelastic solutions like actin, the corner frequency cannot be obtained from its PSD as there is no frequency dependent shift observed in the power spectrum (bottom image in Fig. 5.5). Thus, in these cases, the trap stiffness is obtained from the water PSD, where the same particle size is used for trapping at the same given laser power. 


\section{\begin{tabular}{l|l} 
Chapter 5 & MECHANICS OF ACTIN-DNA SENSOR NETWORKS
\end{tabular}}
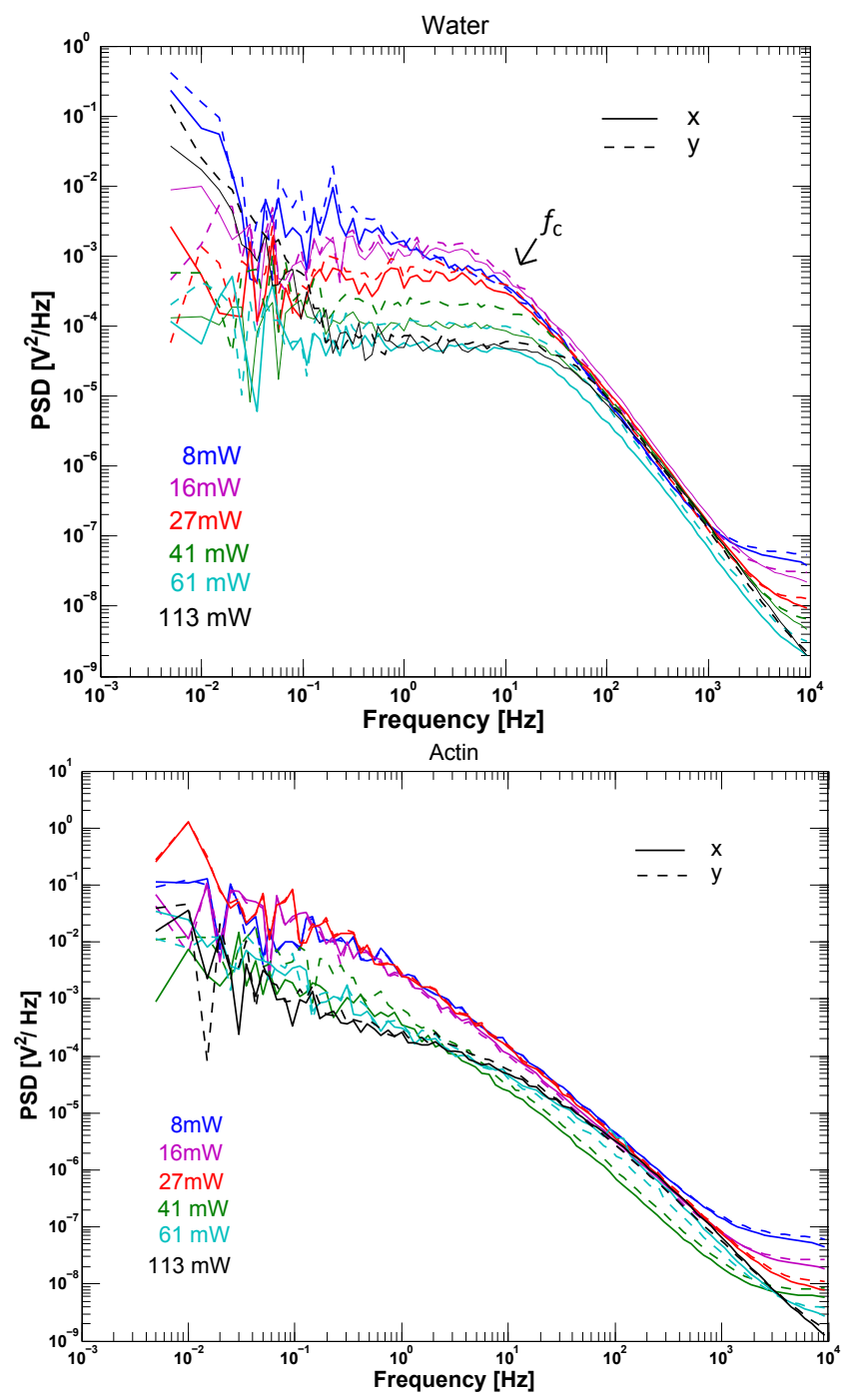

Figure 5.5.: The power spectrum of bead in water and actin at varying laser powers. Autocorrelations of particle displacement in water (above) and $1 \mathrm{mg} / \mathrm{ml}$ actin (below) at various laser powers are given as a function of frequency. The corner frequency $\left(f_{c}\right)$ is indicated by the arrow. In water, $f_{c}$ shifts as laser power increase. Viscoelastic solutions such as actin (bottom image) do not have a shit in the corner frequency. The intensity of the laser $(\lambda=1064 \mathrm{~nm})$ was varied between $8 \mathrm{~mW}$ and $113 \mathrm{~mW}$. PSD refers to power spectral density.

The viscosity of water is calculated from the power spectra represented in Fig. 5.6 where the uncorrected viscosity and the corrected viscosity $\left(G^{\prime \prime}\right)$ are displayed. The effect of trap stiffness can be well seen in the uncorrected $G^{\prime \prime}$, which increases with an increase in the laser power settings. The highest laser power $(113 \mathrm{~mW})$ gives a tight trapping stiffness (upper image in Fig 5.6. The dotted lines indicate the viscosity $\left(G^{\prime \prime}\right)$ of water in the uncorrected frequency spectra. After correcting for the trap stiffness, the pure viscosity of 

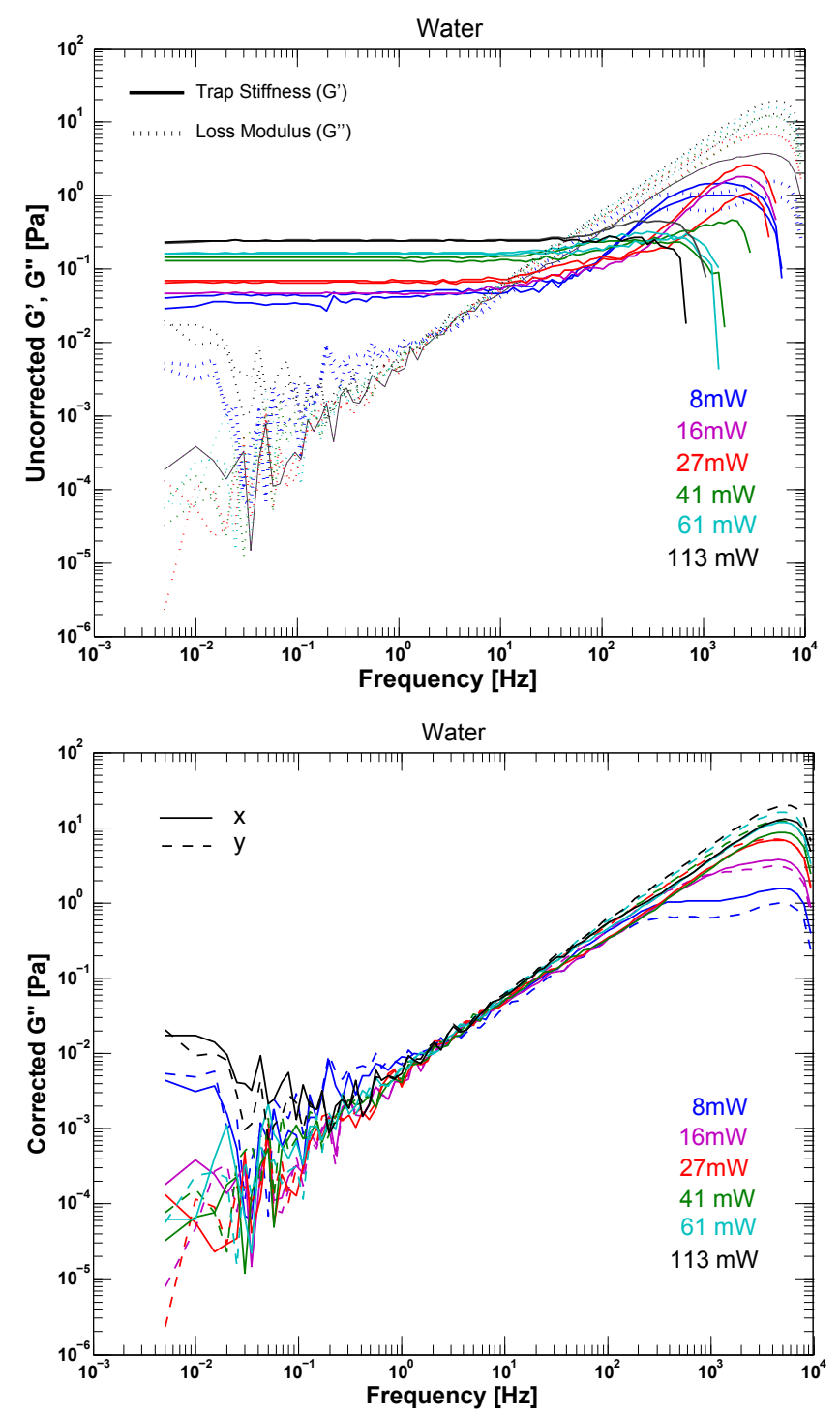

Figure 5.6.: The viscosity of water at different laser powers. The loss modulus ( $\left.G^{\prime \prime}\right)$ of water when uncorrected (top) and corrected (bottom) for trap stiffness is represented for various laser powers. The increasing stiffness in a trap due to increasing laser powers are well seen in the uncorrected G'. Laser powers vary from 8, 16, 27 41, 61 \& $113 \mathrm{~mW}$ which is given in blue, pink, red, green, cyan and black color.

water for all the different laser powers can be observed in the bottom image of Fig. 5.6

The frequency dependent storage $\left(G^{\prime}\right)$ and loss modulus $\left(G^{\prime \prime}\right)$ of viscoelastic actin solutions are obtained from the power spectra in Fig. 5.5. The uncorrected and corrected storage modulus $\left(G^{\prime}\right)$ of entangled actin solutions is represented in Fig. 5.7. The uncorrected storage modulus $\left(G^{\prime}\right)$ in Fig. 5.7 shows the effect of trap stiffness for all laser 


\section{\begin{tabular}{l|l} 
Chapter 5 & MECHANICS OF ACTIN-DNA SENSOR NETWORKS
\end{tabular}}
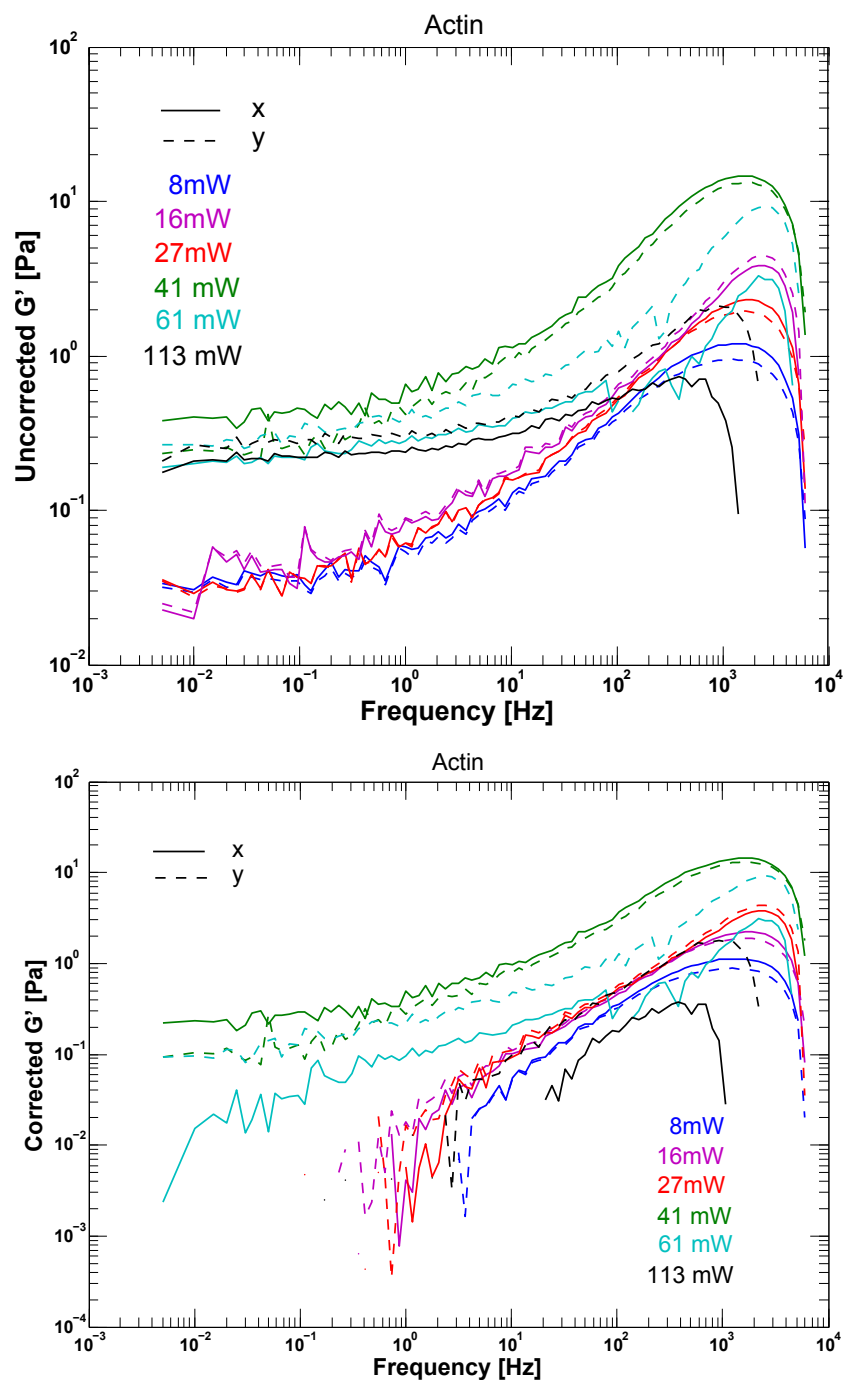

Figure 5.7.: The storage modulus of actin at various laser powers. The storage modulus $\left(G^{\prime}\right)$ of actin are shown for various laser powers when uncorrected for the trap stiffness (above) and when corrected for the trap stiffness (bottom). A $1 \mathrm{mg} / \mathrm{ml}$ concentration of actin was used.

powers. The trap stiffness increases proportionally to the laser intensity except for 113 $\mathrm{mW}$. When corrected for trap stiffness (bottom image in Fig. 5.7), its $\mathrm{G}^{\prime}$, at lower laser powers $(8,16 \& 27 \mathrm{~mW})$, are not the same. These curves lie close to each other but do not converge on the same line. At higher laser powers $(41 \& 62 \mathrm{~mW})$, the effect of trap stiffness can be seen as a plateau at low frequencies (green \& cyan curves) representing an elasticity like that of a crosslinked actin except for the $113 \mathrm{~mW}$ laser power (reason unknown). Thus, the actual storage moduli of entangled actin are seen only at lower laser powers of $8,16 \& 27 \mathrm{~mW}$ (the corrected $\mathrm{G}^{\prime}$ - bottom image in Fig. 5.7). Therefore the frequency spectra of entangled actin at higher laser powers, is only an effect of the trap 

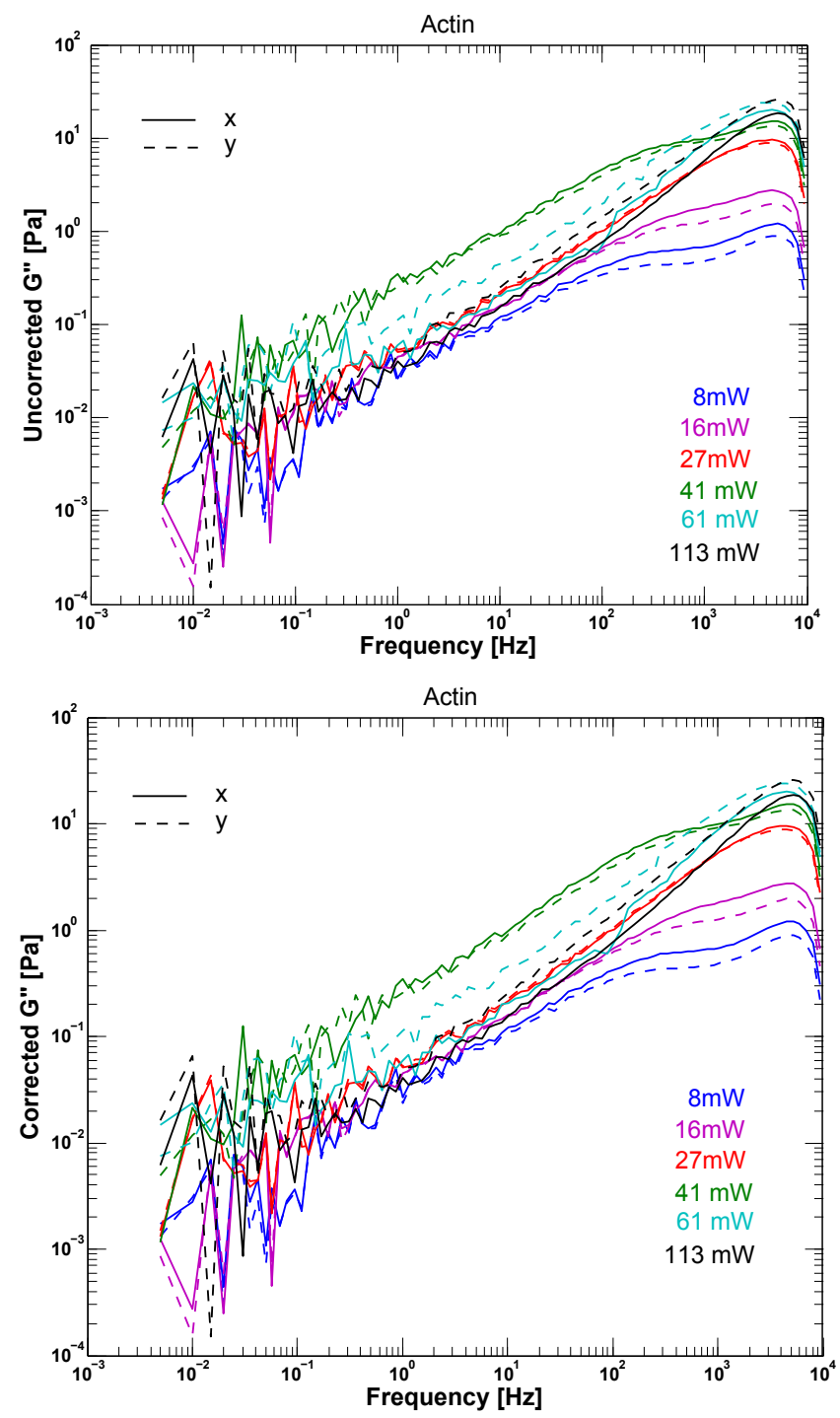

Figure 5.8.: The loss modulus of actin at varying laser powers. The viscous loss modulus $\left(G^{\prime}\right)$ of a $1 \mathrm{mg} / \mathrm{ml}$ actin are shown for different laser powers. Upper plot represents the loss modulus when trap stiffness is uncorrected and the lower plot when it is corrected.

stiffness. The viscous (loss) modulus ( $\left.\mathrm{G}^{\prime \prime}\right)$ of entangled actin network both for the uncorrected and corrected (Fig. 5.8 remains the same without any influence of trap stiffness. This viscosity is the viscosity of water.

\section{Nyquist Criterion: Sampling Frequency Optimization}

We recorded the power spectrum of a trapped bead ( $1 \mu \mathrm{m}$ silica) in water at different sampling frequencies for various laser powers settings. This experiment was carried out to avoid the effects of aliasing and to chose an optimal sampling frequency $\left(f_{s}\right)$. Aliasing of 


\section{\begin{tabular}{l|l} 
Chapter 5 & MECHANICS OF ACTIN-DNA SENSOR NETWORKS
\end{tabular}}
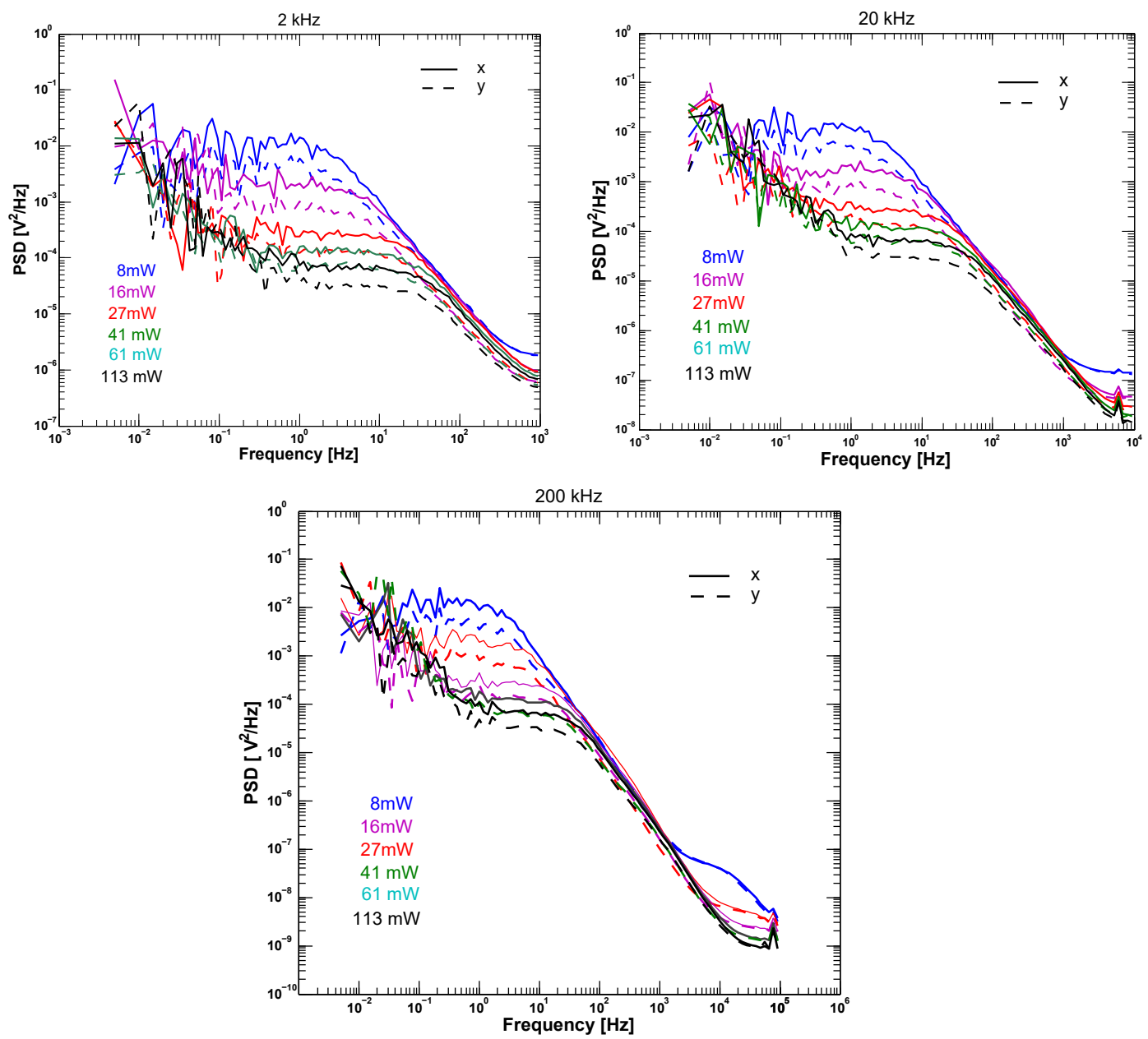

Figure 5.9.: Water PSD at various powers for different sampling frequency. Power spectrum of water for various laser powers sampled at three different frequencies $2,20 \& 200 \mathrm{kHz}$.

a power spectrum occurs in a limited data acquisition bandwidth. This effect was avoided by low pass filtering with a cut off frequency at the Nyquist frequency liii The power spectrum was calculated from the bead displacement. Sampling frequency optimization was performed at 3 different bandwidths 2, $20 \& 200 \mathrm{kHz}$. As observed clearly, a lower sampling rate $(2 \mathrm{kHz})$ cuts off the signal frequency at $1000 \mathrm{~Hz}$. Higher sampling rates of 20 and $200 \mathrm{kHz}$ captures the signal frequency although $200 \mathrm{kHz}$ sampling rate gives a higher noise at higher frequencies. Due to this reason and to reduce large data size, 20 $\mathrm{kHz}$ was chosen as the optimal sampling rate (frequency) for the microrheology on actin and actin-sensor networks.

iii Highest frequency component in the data is equal to $f_{N y q}=0.5 x f_{s}$, where $f_{N y q}$ is Nyquist frequency, $f_{s}$ is sample frequency. In a power spectrum, power spectral density above $f_{N y q}$ will be folded back to low frequencies leading to an alias [51]. 


\subsubsection{Frequency Response of Densely Crosslinked Actin-DNA Sensor Network ( $R=$} $0.1)$
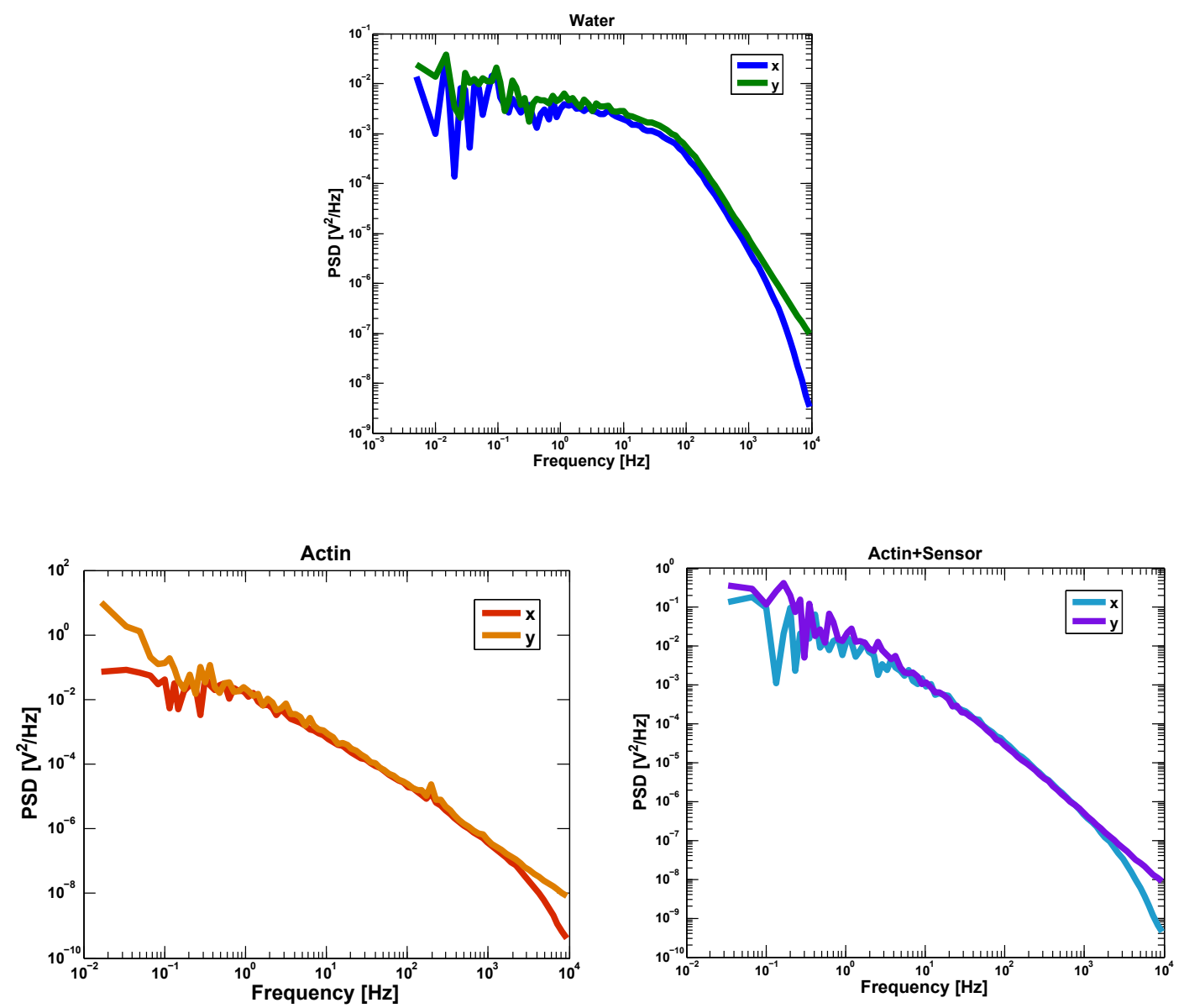

Figure 5.10.: Power Spectra of water, actin, and actin-DNA sensor network. The power spectral density of water (above), actin (bottom left), and actin-DNA sensor network (bottom right) is represented as a function of frequency. PSD curves are represented for the particle displacement in $x$ and $y$-direction. Actin and actin-DNA sensor networks which are viscoelastic solutions do not follow a Lorentzian function as water, where a trap stiffness regime at lower frequencies and the free diffusion of the particle at higher frequencies is observed. Silica particles of $1 \mu \mathrm{m}$ were used.

To probe the local viscoelasticity of actin-DNA sensor networks and entangled actin network, 1 PMR approach was used. $1 \mu \mathrm{m}$ silica particles were used for this purpose. Actin concentration was $1 \mathrm{mg} / \mathrm{ml}$. The optimized $R=0.1$ network from macrorheology experiments ( $\sec 5.2 .1$ ) which has a high elastic shear modulus is used, where $R=$ DNA sensor concentration/actin concentration. Actin solutions and actin-DNA sensor solutions were prepared as stated under the experimental procedure, except that the addition of silica particles in water was done here before the addition of polymerization buffer. From 


\section{\begin{tabular}{l|l} 
Chapter 5 & MECHANICS OF ACTIN-DNA SENSOR NETWORKS
\end{tabular}}
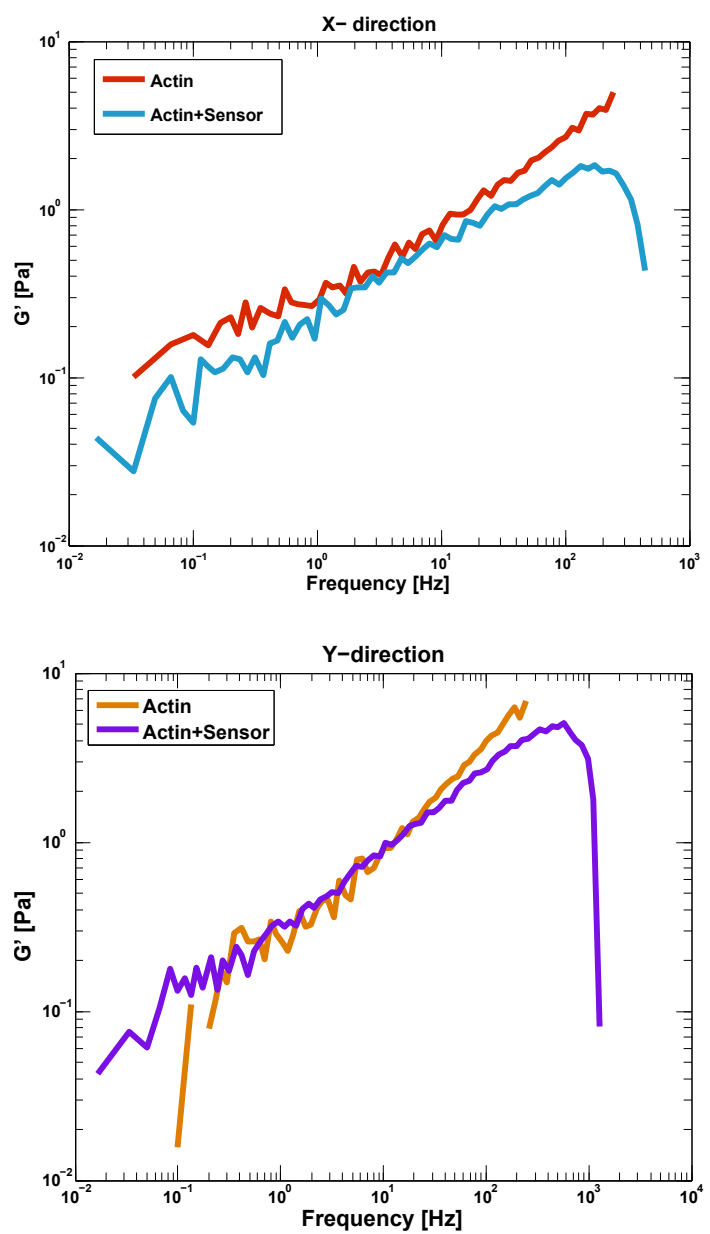

Figure 5.11.: Frequency dependent $G^{\prime}$ of actin and actin-DNA sensor network. The viscoelastic frequency response of an entangled actin network and a crosslinked network of actin - DNA sensor is shown for particle displacement in $x$ and $y$ directions. At $1 \mathrm{~Hz}$ the elastic modulus $\left(\mathrm{G}^{\prime}\right)$ of actin is $0.4 \mathrm{~Pa}$. The crosslinked actin-DNA sensors network has an elasticity lower than actin, where a fluid-like behavior is observed similar to the curve of entangled actin for all probed frequencies.

the above-mentioned optimization experiments, $25 \mathrm{~mW}$ laser power and $20 \mathrm{kHz}$ sampling frequency was found optimal for elucidating the local viscoelastic properties of actin and actin-DNA networks.

The storage and loss moduli determined from displacements of the trapped particle in actin and actin-DNA sensor networks are given in (Fig. 5.11). Measurements were made 2 hours after the polymerization of networks. The entangled actin solutions have an elastic modulus of around $0.4 \mathrm{~Pa}$ at $1 \mathrm{~Hz}$ which is similar to the macrorheology data obtained 5.2.1. There is no discernible effect of a plateau in elasticity for entangled actin probed at all given frequencies. The loss modulus of actin is the same as the elastic modulus. Upon 

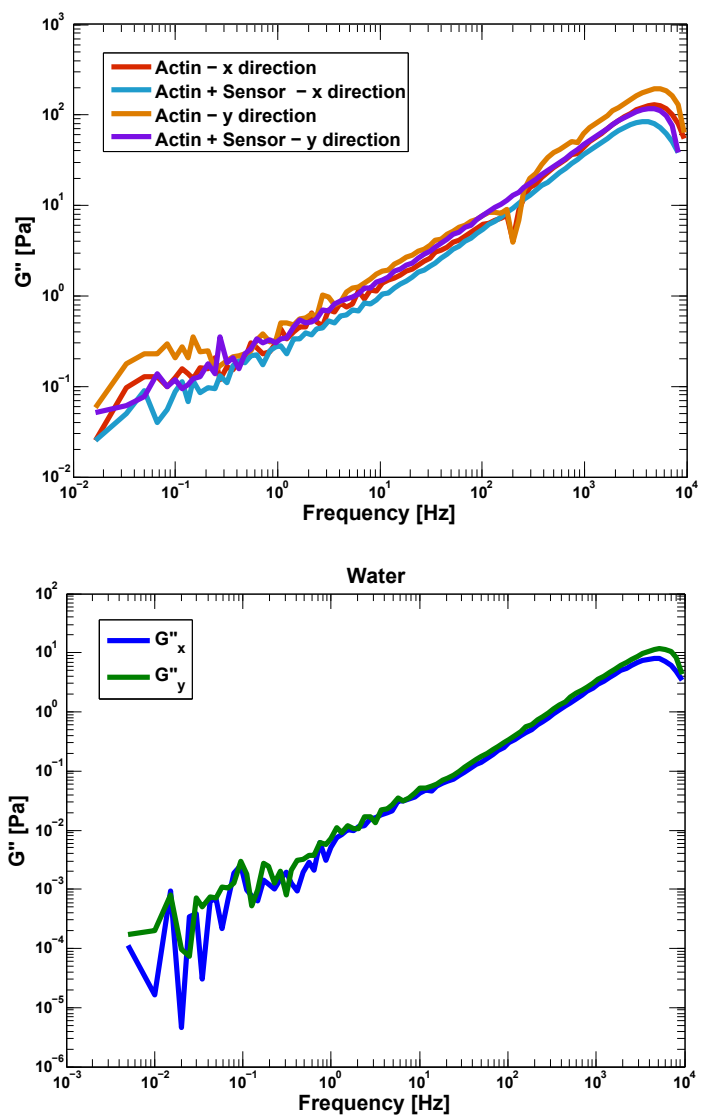

Figure 5.12.: Frequency dependent G" of actin, actin-DNA sensor networks. The viscous loss modulus ( $\left.G^{\prime \prime}\right)$ of actin, actin-DNA sensor network observed is the viscosity of water.

crosslinking actin with the DNA sensors, we do not observe any distinguishing effect of elasticity at low frequencies. Rather the actin-DNA sensor network shows a fluidization behavior at all probed frequencies as against the crosslinked elastic signature expected for a crosslinked network of actin by an ABP observed through microrheology [62].

The elasticity $\left(G^{\prime}\right)$ of actin-DNA networks is slightly lower than actin, which indicates the existence of a liquid-like soft gel. This might be due to the bundle formation that may have occurred in these gels at the end of 2 hours of polymerization which may be the reason for the observed fluidity. The bundling of filaments occurs due to the high sensor concentration $(R=0.1)$. The networks then enter the composite phase of microstructure, where bundles coexist (Fig. 5.4) leading to liquid-like local viscoelasticity of actin-sensor networks. The viscous (loss) modulus of actin and actin-DNA networks follows the same frequency dependent behavior of water and represents the water viscosity, as shown in (Fig. 5.12). The corresponding PSD of actin, actin-DNA networks, and water are shown in Fig. 5.10 


\section{\begin{tabular}{l|l} 
Chapter 5 & MECHANICS OF ACTIN-DNA SENSOR NETWORKS
\end{tabular}}

\subsubsection{Discussion}

In this chapter, the mechanics of crosslinked actin-DNA sensor networks was determined via macro- and microrheology. From the macrorheology, actin-DNA sensor networks show a remarkable six-fold increase in elasticity at the highest sensor concentration. A similar finding [69] reported such an increase in elasticity for actin-DNA networks at the highest DNA concentration. In our results, the elasticity of actin-DNA sensor network for high sensor concentrations $(R=0.1, R=0.2)$ do not reach steady-state after $3600 \mathrm{~s}$. A similar finding was reported by Lorenz et al., [69] for a high DNA concentration in their actin-DNA networks after $2000 \mathrm{~s}$.

The frequency dependent viscoelastic properties of actin and actin-DNA sensor networks were probed through both macro and microrheology. The storage elastic modulus $\left(G^{\prime}\right)$ of actin agrees with the other reports on the frequency response of actin [59], [44] and for other viscoelastic solutions [11]. The crosslinked actin-sensor networks do not show a time-dependent behavior except that they are elastic at low frequencies which is a signature of their crosslinking. They follow a power-law scaling behavior after $1 \mathrm{~Hz}$, which is closer to an actin-palladin network [47].

Microrheological experiments that probe viscoelasticity at the length scales of the network was used in this work to investigate the local viscoelastic behavior of $R=0.1$ actinDNA sensor network. In this experiment, we had observed a fluid-like behavior at the high sensor concentration $(R=0.1)$. Such a fluidization of the actin-DNA sensor network which is due to the excess DNA sensor concentration may be compared to a similar one reported for actin-cofilin networks in [79]. Here, increasing the cofilin concentration resulted in a fluid like beahvior of actin network solutions.

The microstructure of actin-DNA sensor networks in this work is a direct result of crosslinking and not due to other factors such as phase separation or entropically driven network formation [33]. We confirm this by comparing to the previously existing reports on actin-DNA networks. As an example, actin-DNA networks that are entangled and not crosslinked also lead to bundle formation. This is caused entropically by DNA, that drives actin filaments to bundle formation. Such bundle formation occurs at the loss of connectivity of entanglements in actin [33] which is not the case for our crosslinked actin-DNA sensor networks. These remain crosslinked post 1 hour of polymerization.

In another similar finding [139], the appearance of bundles in composite networks of actin-DNA has been reported, which is solely due to phase separation. Upon addition of trivalent ions, actin condenses into bundles and DNA into toroids. While the above two cases are purely studies of actin-DNA networks as coentangled composite networks without any induced crosslinking, our results of a crosslinked network (actin-DNA sensors) 
agree to a similar finding of crosslinked actin-DNA network [69]. They had also utilized lifeact to crosslink DNA to actin, which is the same as ours, except that the attachment chemistry differs. Nevertheless, in a crosslinked actin-DNA network, they [69] observe a composite network structure with bundle appearance at high crosslinker concentration similar to ours. Such a co-existence of bundles in our isotropic crosslinked network arises solely due to the increase in crosslinker concentration as also reported by them and not due to any condensation of ions or an entropically driven bundle formation.

In brief, we have successfully shown the possibility of crosslinking DNA force sensors across a polymeric network of actin with the aid of lifeact (ABP). Such model networks of rigid rods (actin) and flexible linkers (DNA sensors) help recapitulate the cytoskeletal network in vitro for the study of force transmission. These networks can be probed in the future for their non-linear mechanical properties such as stress relaxation mechanism, re-annealing of networks after their rupture at yield stress. With the feasibility of a force sensor across a polymer network, it helps to gain a microscopic picture of the glassy dynamics underlying transient networks. 



\section{Summary \& Outlook}

The presence of internal cellular forces at the molecular scale has been shown to influence various cellular functions [34], [31], [95]. Advances in the field of force sensing have led to the development of a wide variety of force sensing tools. Among them, molecular force sensors have been recently developed which are integrated directly within the biological structure of interest [83], [46], [84] to be probed. As a new class of molecular tension probes, DNA sensors have gained recent popularity [132], [138], [19], [66].

This current work is about our FRET-based molecular DNA stretch sensitive sensor. Through spectrometer measurements, we established the proof of principle of our DNA sensors by quantifying their bulk fluorescence in the quenched and open states. As part of this thesis work, we have developed excellent "controls" that proves the efficiency of our tension sensors by utilizing it in all characterization experiments. Since we aimed to understand force distributions across actin networks, we have utilized the haloTag-fusion protein technology [70] in this thesis to attach our DNA sensors and controls to actin via lifeact.

Through FLIM measurements, we characterized our DNA sensors by determining their lifetimes in the quenched/closed (high FRET) and fluorescent/opened state (low FRET). We searched for a "best quenching" efficiency which is 0.5:1:1 (F:H:Q). That is, we have the most efficient FRET quenching when the F strands are at half the molar concentration 


\section{\begin{tabular}{l|l} 
Chapter 6 & SUMMARY \& OUTLOOK
\end{tabular}}

of the $\mathrm{H}$ and $\mathrm{Q}$ strands. By using this stoichiometry, the sensor was then characterized for its lifetime at zero force (quenched) and threshold opening force (fluorescent) which are $0.5 \mathrm{~ns}$ and $3.6 \mathrm{~ns}$ depending on the buffer. The longer lifetime of $3.5 \mathrm{~ns}$ that is also present in quenched sensors may occur due to the presence of a ground-state complex (static quenching) which needs to be validated in future experiments.

The development of "controls" (sensor structure without the quencher molecule) and determination of their lifetimes indicate that our molecular DNA sensor is indeed a FRET-based sensor. Lifetime measurements (FLIM) and bulk fluorescence quantification (through the spectrometer) reveal that individual F strands \& F+H strands has a certain level of structural quenching present inherently in our sensors. This quenching disappears when the partially assembled sensor $(\mathrm{F}+\mathrm{H})$ is opened by the $\mathrm{C}$ strand, where the characteristic long lifetime is observed as well as an increased high fluorescence. From FLIM measurements, it was observed that the population of DNA sensors that remain quenched in DNA buffer is larger (35\%) than in actin buffer $(24 \%)$. When DNA sensors are completely denatured and annealed in PCR, they do not have a quenched lifetime. From the characterization of DNA sensors, we estimated that our stretch-sensitive DNA sensor has a FRET efficiency of $85 \%$.

Sensors characterization across the actin network was completed in this thesis work. The bulk fluorescence of sensors in their quenched and open states across the actin network was quantified with a spectrometer and through quantitative imaging (confocal laser scanning microscopy). From FLIM, lifetimes of sensors were measured in both of these states. The optimized stoichiometry (0.5:1:1 - F:H:Q) from the results of the solution measurements (Chapter 3) was used in networks for the two different sensor concentrations $(R=0.01 \& R=0.1)$. This gave insight about the population of quenched sensors in both of these networks. In the low sensor concentration network $(R=0.01)$ we observed that only $22 \%$ of sensors are quenched across the network. In the high sensor concentration $(R=0.1)$ we observe a higher amount of quenched sensors ( $36 \%$ ) across the network. What remains unclear as of now from these two observations are, that, if these quenched populations are the ones crosslinked to actin filaments or do they represent quenched sensor population in solution. A possible experiment to help clarify this is to incorporate uncrosslinked sensors into the actin network and determine their lifetimes in the uncrosslinked condition across the actin network. The spatial distribution of sensors across the actin network was also studied through the sensor characterization across the actin network. From the results (Table 4.1. Table 4.4 and Fig 4.9) we observed that a uniform quenching occurs throughout the whole network. This is a notable result, which is later essential for studying the force distribution across the network. This result of homogeneous quenching in the whole network will be helpful later to indicate which part of the network bears forces due to the opening of sensors (non-quenched lifetimes) or where forces distribute when the network is deformed locally with an external force. 
As one of the goals is to measure the force distribution across the cellular cytoskeleton, we tested our sensors by introducing them into live $3 \mathrm{~T} 3$ fibroblasts at $20^{\circ} \mathrm{C}$ and measured the lifetime of our sensors via FLIM. We also introduced "controls" inside live fibroblasts and measured their lifetime. Comparing sensors mean lifetime ( $2.7 \pm 0.5 \mathrm{~ns})$ and controls lifetime mean lifetime ( $\sim 4 \mathrm{~ns}$ ) inside live fibroblasts, we find promising evidence of our DNA sensors function, amidst the complex cellular environment. These findings need to be further validated with multiple control experiments in the future to precisely measure lifetimes across a specific actin structure. From the RFP lifeact images, we prove the colocalization of DNA sensors on actin structures. These results point out a future possibility that our DNA sensors can be attached to other different actin architectures inside the cell. This can be achieved by modifying the attachment ends of sensors with several other actin-binding proteins apart from lifeact. Sensors with different lengths of hairpins (hairpin 16 and 24) along with hairpin 8 can also be introduced into cells to simultaneously probe multiple force ranges. This can be achieved by using distinct FRET pairs for each hairpin length such that each sensor lights up for different forces.

Attaching DNA sensors to actin filaments in vitro, resulted in an elastic crosslinked network. This was evident from their elastic shear modulus $\left(G^{\prime}\right)$ in the macrorheology experiment. Morphologically, these model networks have an isotropic crosslinked network structure at low sensor concentrations but transitions to a composite network structure at high sensor concentrations where bundles develop. Using two different stoichiometries of F:H:Q strands (the 1:1:1 and the 0.5:1:1), we arrived at networks having two different stiffness in which 0.5:1:1 networks are less stiff than 1:1:1. Actin-DNA sensor networks are viscoelastic, but they do not exhibit a frequency dependent behavior (i.e their elastic shear moduli G', does not show a frequency dependent change in the linear regime (small amplitude deformation)). Contrary to the macrorheology, the microrheology based frequency behavior of the $R=0.1$ actin-DNA sensor network indicated a fluid-like behavior after one hour of polymerization. The local elastic modulus $G^{\prime}$ of this network was lower than the $G^{\prime}$ of the entangled actin network.

In conclusion, this study has successfully determined the FRET efficiency of our molecular DNA tension sensors. The quencher-lifetime determination at zero force will enable in future to do force mapping and imaging of force fields in actin networks through FLIM. It may be also possible to investigate the re-annealing mechanisms in these transient networks, after they have ruptured at a given yield stress. An interesting prediction from theory and simulations in networks, is the development of force chains due to anisotropic strain distributions [49],[8]. We hope that this work of characterization of sensors in the actin network, may later help in the study of force chains if present in any model network like actin-DNA sensor network. Thus, we hope this work would contribute in the future to a larger understanding of network mechanics in the non-linear regime and also in the study of force distributions across the cytoskeleton of live cells. 



\section{Appendix - Protocols}

\section{A.1 Biochemical Protocols}

Materials:

- HaloTag Iodoacetamide $\left(\mathrm{O}_{4}\right)$ Ligand (Promega, Madison, USA)

- Atto 647N Phalloidin (Atto-Tec GmbH, Siegen, Germany)

- DNA oligos (Integrated DNA Technologies, Leuven, Belgium)

- TCEP (Tris(2-carboxyethyl) phosphine hydrochloride) (Sigma-Aldrich, Germany)

- SYBR green nucleic acid gel stain (Molecular Probes, Invitrogen)

- Polymix 10X (Cat.\# 5000-01, Hypermol, Bielefeld, Germany)

- 20\% TBE Gel 15 wells (Novex, Fisher Scientific EC63155 Box, Germany)

- 1X TBE running buffer

- DNA loading dye (Thermo Scientific, R0611, Germany)

- Gene Ruler ultra low range DNA ladder (Life Technologies GmbH, Darmstadt, Germany) 
- Intas ECL chemocam imager (INTAS Science Imaging Instruments GmbH, Sartorius, Göttingen, Germany)

- Cuvette (Type No. 105.253-QS, Hellma Analytics, Germany)

\section{A.1.1 KOH Cleaning}

- Place microslides (631-1550, VWR, Germany), coverslips (No. 1.5, 24×24 mm, VWR,Germany) in a teflon holder in a glass box.

- Add few drops of $1 \mathrm{M} \mathrm{KOH}$.

- Fill the glass box with $99 \% \mathrm{EtOH}$ so that the mircoslides, coverslips are fully submerged.

- Sonicate for $5 \mathrm{~min}$.

- Discard $\mathrm{KOH}$ solution and sonicate the coverslips 2 times in MilliQ water.

\section{A.1.2 Preparation of Chambers for Actin Networks}

- To the cleaned microslides, stick $75 \mu \mathrm{m}$ thick 3M double stick adhesive tapes each 3 $\mathrm{mm}$ wide in the middle of the slide.

- Place the appropriate actin/actin-DNA sensor solution between the sticky tapes.

- Cover immediately with the $\mathrm{KOH}$ cleaned coverslips. Avoid air bubbles while smushing it down.

- Seal all the four sides with VALAP and cover with aluminium foil.

- Allow the solution to polymerize into networks for 1 hour. 


\section{A.1.3 Actin and Actin-DNA Sensor Network Sample Preparation}

- G-actin used for this work was self prepared according to the protocols of [36],[101],[75].

- It was prepared from rabbit skeletal muscle and was stored as small aliquots in -80 ${ }^{\circ} \mathrm{C}$.

- Each aliquot is a single use, thawed freshly for the preparation of actin and actinDNA sensor networks.

- $10 \%$ volume of polymerization buffer Table A.1 is added to an appropriate volume of water and then mixed with an appropriate volume of G-actin $(1 \mathrm{mg} / \mathrm{ml})$.

- $10 \%$ volume of Atto $647 \mathrm{~N}$ phalloidin is added to the above mixture, mixed well by pipetting up and down gently with a pipette and then added onto the microscopic slide.

- Approximateley $50 \mu \mathrm{l}$ of above solution is loaded on to each chamber, closed with coverslips and sealed with VALAP.

- Solutions of actin/actin-DNA sensors are allowed to polymerize for 1 hour.

\section{A.1.4 Preparation of Sensor Strands F and Q}

- Lyophilized DNA oligos (Integrated DNA technologies, Leuven, Belgium) was reconstituted in DNA buffer. This was used as the stock solution of DNA oligos.

- DNA oligo strands F and $\mathrm{Q}, 30 \mu \mathrm{M}$ each, were dissolved from the stock solution in DNA hybridization buffer.

- $2 \mathrm{mM}$ TCEP solution was added to each of the above solution.

- They were incubated for about 60 - 90 mins.

- $20 \mu \mathrm{M}$ of iodoacetamide haloligand was then added to the two respective incubated solution mixture.

- They were incubated again for about 60 mins.

- Finally $30 \mu \mathrm{M}$ of the genetically expressed lifeact-RFP-haloTag protein was added to each of the above F and Q DNA oligos. 


\section{A.1.5 VALAP Recipe}

- Equal weight of petroleum jelly (e.g. Vaseline), lanolin and paraffin were mixed together in a 1-L beaker.

- Mixture was kept at a low setting on hot plate.

- It was stirred occasionally until thoroughly blended, care was taken to not overheat this mixture.

- Mixture was warmed enough until components liquefied and the final color of the product was golden yellow color (corn color).

- It was stored at room temperature in small aliquots and was used as sealant.

\section{A.1.6 PCR Annealing and PAGE Electrophoresis}

1X TBE running buffer for electrophoresis:

- $10.8 \mathrm{~g}$ Tris base $(89 \mathrm{mM})$

- $5.5 \mathrm{~g}$ Borate $(89 \mathrm{mM})$

- 0.7 g EDTA (2 mM)

- The above constitents were dissolved in $1 \mathrm{~L}$ water at $\mathrm{pH} 8.0$

PCR and room temperature annealing:

- FHQ strands each $100 \mu \mathrm{M}$ in concentration was mixed to a final volume of $2 \mu \mathrm{l}$.

- FHQ, FHQC, FH, FHC, QH, QHC and HC were the combination of strands.

- PCR annealing of strands involved denaturing DNA strands at $95^{\circ} \mathrm{C}$ and re-annealing by rapid cooling as mentioned in Table A.4.

- Room temperature annealing involved mixing the strand combinations at the given molar concentration at room temperature.

PAGE electrophoresis of annealed sensors:

- $2.5 \mu \mathrm{l}$ of PCR annealed sensors and room temperature annealed sensors were individually mixed with $247.5 \mu \mathrm{l}$ of DNA buffer.

- $2.5 \mu \mathrm{l}(\approx 250 \mathrm{nM})$ of the above solution was mixed with $7.5 \mu \mathrm{l}$ of water \& $2 \mu \mathrm{l} 6 \mathrm{X}$ DNA loading dye . 
- The above mixture was loaded into wells and run for $1 \mathrm{~h}$ with $200 \mathrm{~V}$ at room temperature .

- Gels were then stained with SYBR Gold (1:10000 diluted) in TBE for 1h at room temperature.

- They were then imaged in UV fluorescence. 
Appendix | APPENDIX-PROTOCOLS

Table A.1. Actin polymerization buffer - Polymix 10X

\begin{tabular}{l|c} 
Constituents & Concentration (in mM) \\
\hline $\mathrm{KCl}$ & 100 \\
Immidazol (pH 7.4) & 10 \\
Adenosine Triphosphate (ATP) & 1 \\
$\mathrm{MgCl}_{2}$ & 2
\end{tabular}

Table A.2. DNA hybridization buffer

\begin{tabular}{l|c} 
Constituents & Concentration (in $\mathbf{m M})$ \\
\hline $\mathrm{NaCl}$ & 50 \\
$\mathrm{Mgcl}_{2}$ & 10 \\
$\mathrm{PBS}$ & $1 \mathrm{x}$
\end{tabular}

Table A.3. DNA oligos sequence

\begin{tabular}{l|l} 
DNA oligo strands & Sequence \\
\hline $\mathrm{F}$ & ${\text { 3' Thiol C3 GCGGGACTTTCGTGCGTCGC Alexa } 4885^{\prime}}^{\prime}$ \\
$\mathrm{Q}$ & 3' Iowa blackFQ CGCGCCCGTGCGCCGAACGC C6 Thiol 5' $^{\prime}$ \\
$\mathrm{H}$ & GCGAACCG GAGAGTGTTAGAGACA CGGTTCGC \\
$\mathrm{C}$ & CTCTCACAATCTCTGTCGGTTCGC
\end{tabular}

Table A.4. PCR annealing of DNA strands

\begin{tabular}{l|l} 
Temperature $\left(^{\circ}\right)$ & Time (min) \\
\hline 95 & 5 \\
80 & 5 \\
70 & 5 \\
60 & 5 \\
50 & 5 \\
40 & 5 \\
30 & 5 \\
20 & Stop
\end{tabular}


DNA strand with Alexa 488

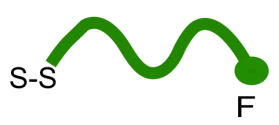

DNA strand with quencher
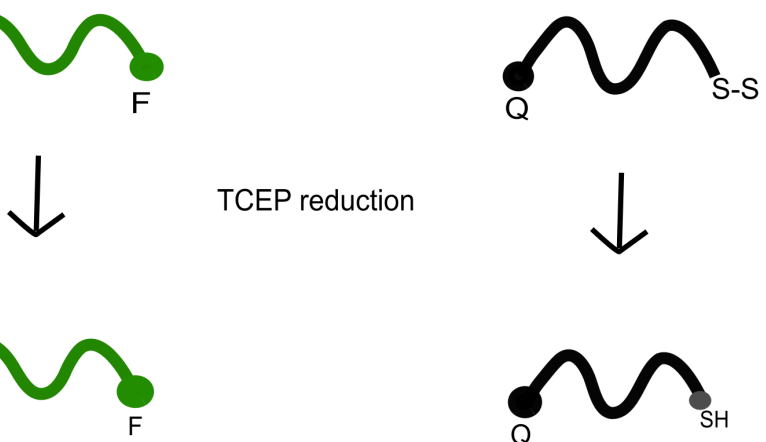

TCEP reduction<smiles>CCCC</smiles>

$\bigcap_{Q}$

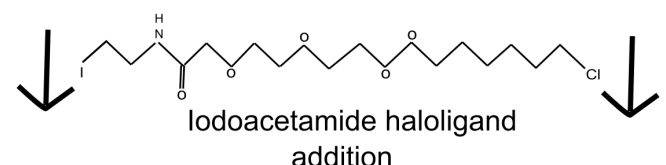

N

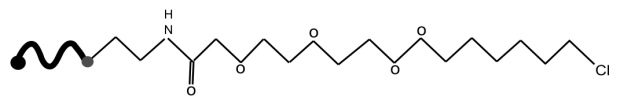

\section{$\downarrow \sqrt{8}$ Halotag Protein with lifeact addition}
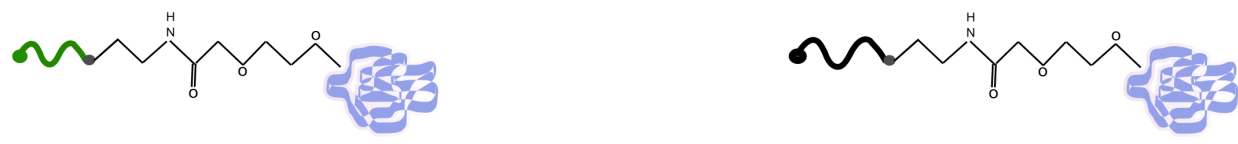

Figure A.1.: Sensor preparation. 


\section{A.2 Cell Culture Protocols}

In this appendix the passaging and transfection of cell are given in two different steps.

\section{A.2.1 Passaging of Cells}

\section{Cell line:}

- 3T3 (ACC 173) mouse embryonic fibroblasts.

\section{Materials:}

- Cell Culture medium: Dulbecco's modified Eagle's medium, High Glucose (DMEM) (D6429, Sigma- Aldrich, St.Louis, MO, USA) with $10 \%$ fetal bovine serum (FBS), heat inactivated $\left(30 \mathrm{~min}, 56{ }^{\circ} \mathrm{C}\right),($ \# F0244, Sigma-Aldrich) and $1 \%$ penicillin-streptomycin (\# 17-602E, Lonza, Basel, Switzerland).

- EDTA/Trypsin solution, 0.05 \% (59417C, Gibco, Thermo Fisher).

- T75 culture flask $\left(75 \mathrm{~cm}_{2}\right)$ (83.1813, Saarstedt AG, Numbrecht, Germany).

- Incubator with $5 \% \mathrm{CO}_{2}$ at $37^{\circ} \mathrm{C}$.

\section{Cell passaging protocol:}

- The medium was removed from a $70 \%-90 \%$ confluent flask.

- $1.2 \mathrm{ml}$ trypsin was added and incubated for $5 \mathrm{~min}$ at $37^{\circ} \mathrm{C}$ ca $3 \mathrm{~min}$.

- After making sure the cells were completely detached, $9 \mathrm{ml}$ medium was added.

- This cell solution was filled up in a $15 \mathrm{ml}$ falcon tube.

- It was centrifuged for $5 \mathrm{~min}$ at $1000 \mathrm{rpm}$.

- Cell pellets were resuspended in $1 \mathrm{ml}$ medium.

- They were counted and seeded in T75 flask with $10 \mathrm{ml}$ medium and in ibidi petridishes with $2 \mathrm{ml}$ medium for imaging. 


\section{A.2.2 Double Transfection of Cells}

DNA sensors were transfected in a two step manner. Firstly the lifeact-RFP-HaloTag was nucleofected on the first day. After 24 hours post transfection, a second transfection step consisting of the assembled sensors is done with viafect.

\section{Cell line:}

- 3 T3 (ACC 173, Leibniz Institute DMSZ, Braunschweig, Germany) mouse embryonic fibroblasts $(50,000$ cells $)$

\section{Materials:}

- PBS (Phosphate buffer saline).

- Cell Culture medium: Dulbecco's modified Eagle's medium, High Glucose (DMEM) (D6429, Sigma- Aldrich, St.Louis, MO, USA) with $10 \%$ fetal bovine serum (FBS), heat inactivated $\left(30 \mathrm{~min}, 56^{\circ} \mathrm{C}\right)$, (\# F0244, Sigma-Aldrich) and $1 \%$ penicillin-streptomycin (\# 17-602E, Lonza, Basel, Switzerland).

- Medium for imaging: $\mathrm{CO}_{2}$ independent medium (Gibco, 1X, 18045-05, Germany), with $10 \%$ fetal bovine serum (FBS), heat inactivated $\left(30 \mathrm{~min}, 56{ }^{\circ} \mathrm{C}\right),($ \# F0244, SigmaAldrich) and $1 \%$ penicillin-streptomycin (\# 17-602E, Lonza, Basel, Switzerland)

- EDTA/Trypsin solution, 0.05 \% (59417C, Gibco, Thermo Fisher).

- Ibidi Petridishes ( $\mu$-dish,35 mm low, 80136, Ibidi GmbH, Martinsried, Germany).

- Incubator with $5 \% \mathrm{CO}_{2}$ at $37^{\circ} \mathrm{C}$.

- Viafect TM Transfection Reagent (44981, Promega, USA)

- 4D Nucleofector Core Unit (AAF-1002B, Lonza, Switzerland).

- 4D Nucleofector X Unit (AAF-1002X, Lonza, Switzerland).

\section{Transfection solution:}

- $82 \mu \mathrm{l}$ of SE Cell Line 4D-Nucleofector X kit L Solution (V4XC-1024, Lonza, Switzerland)

- $18 \mu$ of Supplement 1 solution in the 4D-Nucleofector X kit L (V4XC-1024, Lonza, Switzerland)

- $2 \mu \mathrm{g}$ of pFc14A Lifeact-RFP-haloTag plasmid (home prepared)

\section{Lifeact-RFP- haloTag transfection:}

- Medium was removed from T 75 flasks. 
- $10 \mathrm{ml}$ PBS was added to the the flask.

- PBS was removed, and $3 \mathrm{ml}$ warm Trypsin was added, incubated for $3 \mathrm{~min}$ at $37^{\circ} \mathrm{C}$ and $5 \% \mathrm{CO}_{2}$.

- Cells were completely detached, and $5 \mathrm{ml}$ DMEM was added to stop the trypsin reaction.

- The cell suspension was transferred to $14 \mathrm{ml}$ Falcon-tube.

- It was centrifuged for $5 \mathrm{~min}$ at $1000 \mathrm{rpm}$ and supernatant was discarded.

- $5 \mathrm{ml}$ PBS was added to the cell pellet and centrifuged again for $5 \mathrm{~min}$ at $1000 \mathrm{rpm}$.

- Pellet was re-suspended in $100 \mu \mathrm{l}$ of transfection solution mix and cells were transfected with pulse code DS-150.

- After transfection, the transfected solution was incubated for 3-5 min at room temperature (RT).

DNA sensors \& controls transfection with Viafect (TM):

- $190 \mu$ LMEM medium (plain i.e without FBS and antibiotics), 2 mg of sensors \& controls (synthesized as stated in A.1 without the haloTag protein \& pre-assembled) and $10 \mu \mathrm{l}$ of Viafect TM transfection reagent (Promega, USA) were all mixed well together and added to an Ibidi petridish.

- They were incubated for a period of 5-20 min, but not longer than $20 \mathrm{~min}$.

- Meanwhile cells were rinsed 2 times with PBS.

- Then 0.8-1.0 ml of plain medium was added to cells.

- $200 \mu \mathrm{l}$ of solution that contains viafect \& DNA mixture was added gently from the sides (wall) of the Ibdi petridishes and mixed by gently swirling the dishes.

- They were then incubated for 2 hours and new medium was added. 


\section{A.3 Protein Expression and Purification}

The lifeact-RFP-Halotag is genetically fused and expressed as a protein to be used in actinDNA sensor networks. Hence in this appendix the protein expression in E.coli cells and their purification is given.

Materials required:

Lysis buffer:

- $50 \mathrm{mM}$ Tris $\mathrm{pH} 8.0$

- $250 \mathrm{Mm} \mathrm{NacL}$

- $10 \mathrm{mM} \beta$-Mercaptoethanol

- Protease inhibitor Sigma (1 Tab/100 ml) or 1mM PMSF (freshly made 200x stock)

- $1 \mathrm{mg} / \mathrm{ml}$ Lysozyme

Wash buffer:

- 50 mM Tris pH 8.0

- $250 \mathrm{mM} \mathrm{Nacl}$

- $10 \mathrm{mM} \beta$-Mercaptoethanol

- 20/40 mM Imidazol

- 1 mM PMSF

Elution buffer:

- $50 \mathrm{mM}$ Tris $\mathrm{pH} 8.0$

- 250 mM Nacl

- $10 \mathrm{mM} \beta$-Mercaptoethanol

- 350 mM Imidazol

- 1 mM PMSF 


\section{Appendix | APPENDIX - PROTOCOLS}

\section{Transformation and growth of cell}

\section{Cell growth protocol:}

- Transform BL21 cells with pEt28alifeact-RFP-Halotag-His6 and plate on $\mathrm{LB}_{\text {kan }}$ on 37 ${ }^{\circ} \mathrm{C}$.

- Innoculate $50 \mathrm{ml} \mathrm{LB}$ Kan with a single colony on $37^{\circ} \mathrm{C}$ as a preculture.

- Innoculate $11 \mathrm{LB}_{\text {antibiotics }}$ with $5 \mathrm{ml}$ preculture, and start measuring optical density $(\mathrm{OD})_{600}$ nearly 0.1 .

- Grow until $\mathrm{OD}_{600} 0.4$ to 0.5 at $37^{\circ} \mathrm{C}$.

- Set the shaker to $22^{\circ} \mathrm{C}$.

- Induce with $0.1 \mathrm{mM}$ IPTG.

- Expression for o/N at $22{ }^{\circ} \mathrm{C}$.

- Spin down at $4600 \mathrm{xg}$ for $20 \mathrm{~min}$ at $4{ }^{\circ} \mathrm{C}$, split in $2 \times 500 \mathrm{ml}$ tubes..

- Freeze the pellet at $-80^{\circ} \mathrm{C}$ or resuspend in $20 \mathrm{ml}$ lysis buffer per tube and then freeze in liquid $\mathrm{N}_{2}$ and store at $-80^{\circ} \mathrm{C}$. 


\section{Protein Purification}

Materials required:

- NiNTA: Machery and Nagel Protino NiNTA Agarose (LOT: 1411/002)

- Bradford: Roti-Quant 5x Concentrate (Art.Nr. K015.1)

- PMSF: Applichem powder, stock $200 \mathrm{mM}$ in $\mathrm{EtOH}$ (35 mg in $1 \mathrm{ml}$ ) on ice

- Gelstain: Roti-Blue quick ready to use 1x solution (Art. Nr. 4829.2)

\section{NINTA Purification:}

- Thaw and refreeze in liquid N2 2x and incubate 30 min with lysozym and DNAseA.

- Thaw and sonicate $4 \times 20$ sec output 40 , pulse $50 \%$ on ice.

- Pass 3X through a G 20 Needles.

- Spin in JA30.5 Beckmann rotor $25000 \mathrm{xg} 20 \mathrm{~min} 4{ }^{\circ} \mathrm{C}$.

- Give $2 \mathrm{ml}$ NINT flurry to a $10 \mathrm{ml}$ column, wash with $10 \mathrm{ml} \mathrm{H}_{2} \mathrm{O}$ then with $10 \mathrm{ml}$ Lysis buffer

- Let drain by gravity.

- Fill the supernatant to a $50 \mathrm{ml}$ tube after spinning add the NINTA by transferring it with a few supernatant and incubate by rotation in the cold room for $1 \mathrm{~h}$.

- Add stepwise to the column, let drain by gravity.

- Wash with $10 \mathrm{ml}$ wash buffer.

- Elute with 6x $1 \mathrm{ml}$ Elution buffer in $1.5 \mathrm{ml}$ cups.

\section{Bradford:}

- Make 1x Roti-Quant solution

- Standards with BSA

- $1 \mathrm{ml}$ dye and $20 \mu \mathrm{l}$ probe (may be dilutions)

- $\mathrm{OD}_{595 \mathrm{~nm}}$

\section{SDS PAGE:}

- 10 or $12 \%$ Bis/Tris-Gel precast from Invitrogen

- 1x MOPS running buffer, $500 \mathrm{ml}$

- $100 \mathrm{~V}, 50 \mathrm{~min}$

- Stain with $20 \mathrm{ml}$ Roti-Blue Quick (re-use solution 3-4x, store dark at RT) 

B

Supporting Information 
B.1 Spectrometer: Bulk Fluorescence of Sensor and Controls across Actin $(1: 1: 1-F: H: Q)$

\section{$1: 1: 1(\mathrm{~F}: \mathrm{H}: \mathrm{Q})$}

a) Actin + DNA Sensors

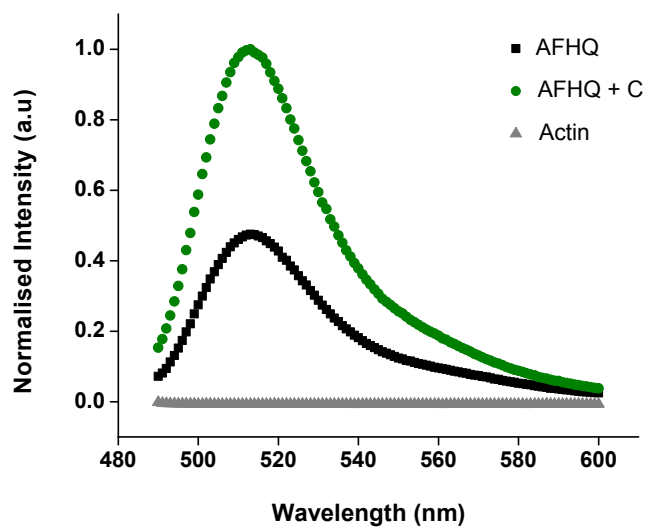

b) Actin + Controls

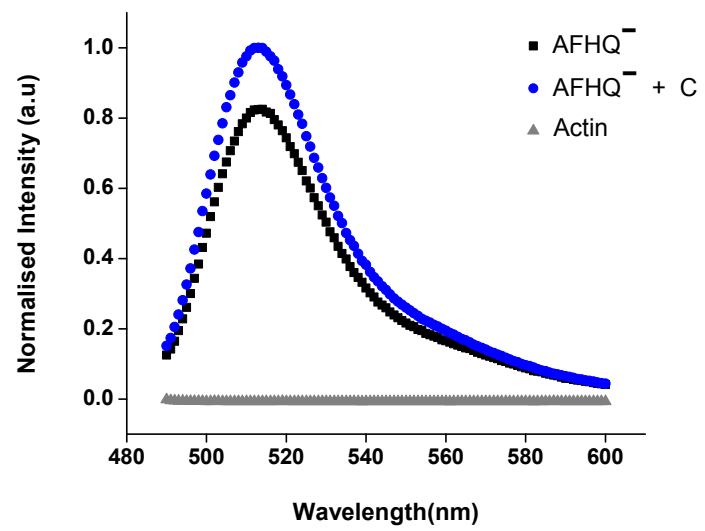

Figure B.1.: Fluorescence of DNA force sensor versus controls when crosslinked to actin $(1: 1: 1-F: H: Q)$. Emission spectra of force sensors and controls crosslinked to actin filaments. a) Working of force sensors in actin (1:1:1- F:H:Q). b) Working of controls in actin $(1: 1: 1-\mathrm{F}: \mathrm{H}: \mathrm{Q})$. Gray line represents uncrosslinked actin (entangled) intensity. F: Strand with fluorophore Alexa 488, H: Hairpin strand, Q: Q strand with quencher molecule, C: Control strand. A: Actin. AFHQ: Quenched sensors in actin, AFHQC: Opened sensors (with $C$ strand) in actin. AFHQ ${ }^{-}$: Closed controls in actin. $\mathrm{AFHQ}^{-} \mathrm{C}$ : Opened controls in actin (opened by $\mathrm{C}$ strand). The values plotted are averaged for $n=5$.

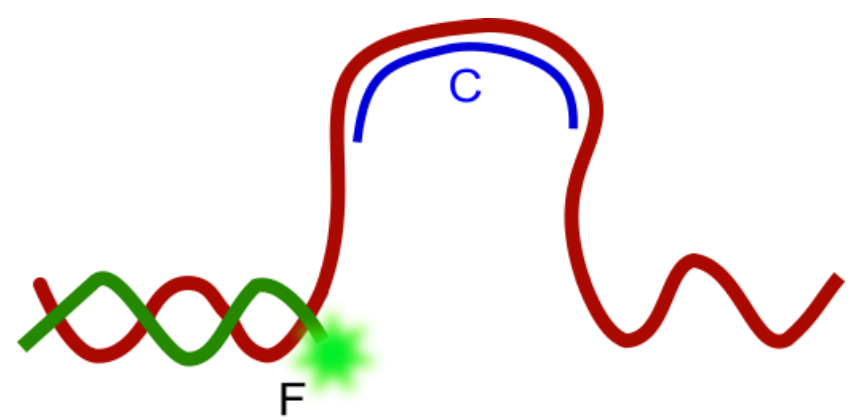

Figure B.2.: Scheme of a partially assembled sensor. A partially assembled sensor that is held opened by $\mathrm{C}$ strand $(\mathrm{F}+\mathrm{H}+\mathrm{C})$ is depicted. Red strand indicates the hairpin strand. 
B.2 Confocal Laser Scan: Quenching and Fluorescence of Sensors across the Network (1:1:1 - F:H:Q)

Quenched Sensors in Actin Network

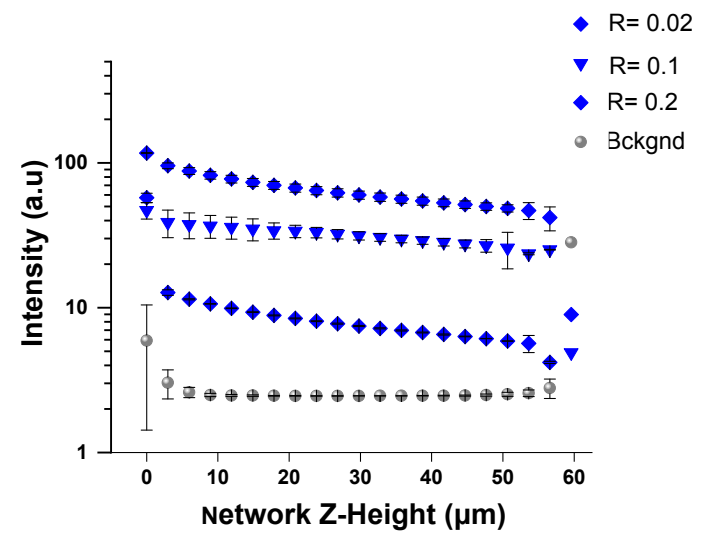

Opened Sensors in Actin Network

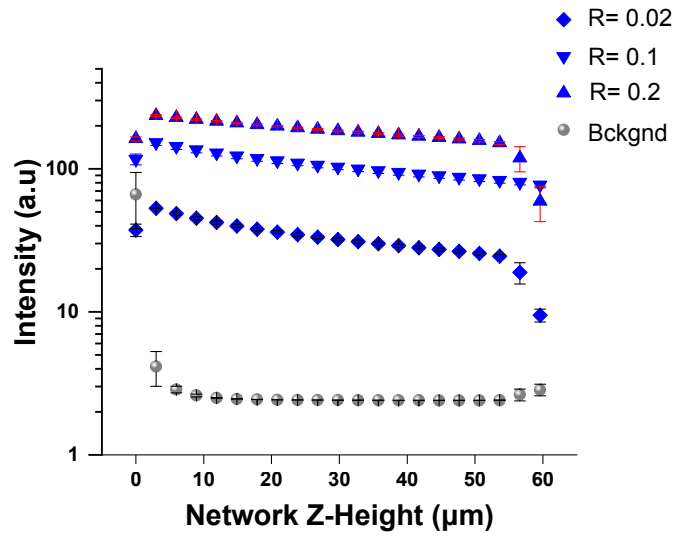

Figure B.3.: Quenched and fluorescence intensity of sensors in actin network 1:1:1-F:H:Q. Quenched sensors and open fluorescent sensors across network for different sensor concentrations (given as $R$ values) are represented for $1: 1: 1-\mathrm{F}: \mathrm{H}: \mathrm{Q}$ stochiometry. A z-stack scan across the entire network was done at 10 different positions. Sensors for all concentrations remains well quenched (on the left) and show a high fluorescence when opened (on the right). For $R=0.005, R=0.01$ networks, we do not show the quenched and open fluorescence as they are at the detection limits of the detector and have high background fluorescence.

Closed Controls in Actin Network

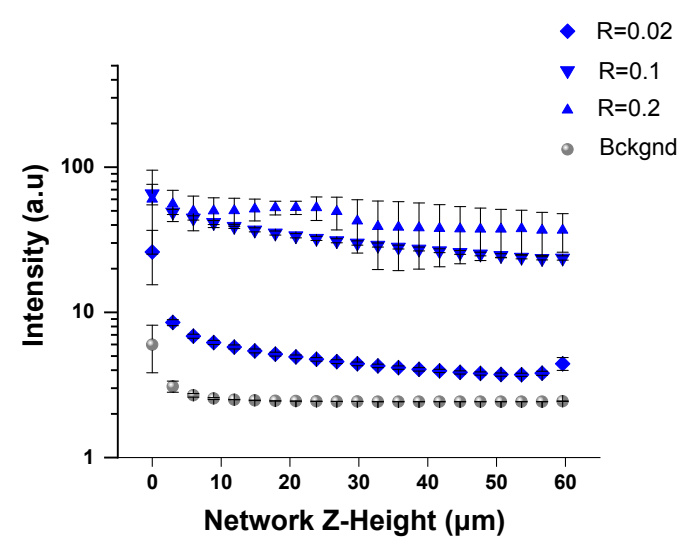

Opened Controls in Actin Network

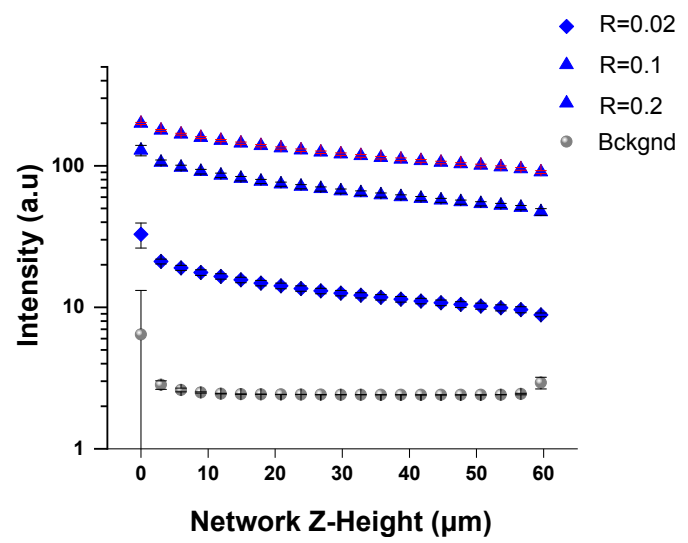

Figure B.4.: Controls fluorescence intensity in actin network for 1:1:1-F:H:Q. Controls closed and open fluorescence across network depicted for different sensor concentrations at 1:1:1$\mathrm{F}: \mathrm{H}: \mathrm{Q}$ stochiometry. A z-stack scan was made throughout the network at 10 different positions. For $R=0.005, R=0.01$ networks, we do not show the controls fluorescence as they are also like sensors within the detection limits of the detector. 


\section{B.3 FLIM: Actin-DNA Sensor Network Equal stoichiometry (1:1:1- $\mathrm{F}: \mathrm{H}: \mathrm{Q})$}

\section{B.3.1 FLIM: Sparsely Crosslinked Network $R=0.01$}

We also performed lifetime measurements in actin-DNA sensor networks for equal stoichiometry (1:1:1- F:H:Q) conditions at two sensor crosslink ratios $(R=0.01, R=0.1)$ in quenched and opened sensors.

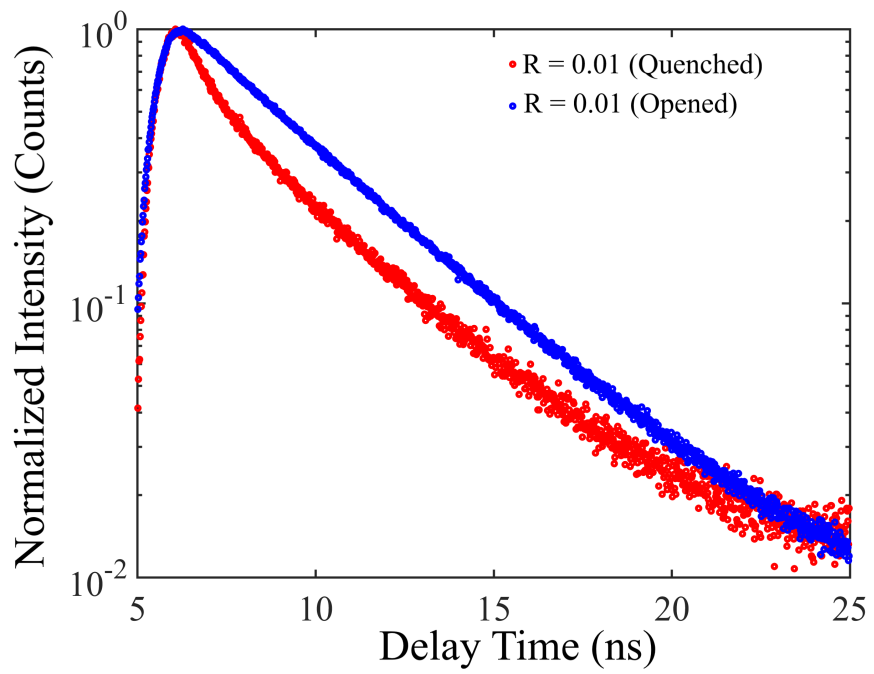

Figure B.5. Lifetime decay curves of $R=0.01$ actin-sensor network 1:1:1 (F:H:Q) stoichiometry. Red curve indicates the quenched sensors across actin network with a bi-exponential component. The small bent region in red curve indicates the short decay of photons due to quenching. Opened sensors across the actin network has a mono-exponential long lifetime decay (blue curve).

Table B.1. Quenched sensor lifetime across actin network $R=0.01$.

\begin{tabular}{lll}
\hline Network height $(\mu \mathbf{m})$ & $\tau_{1}(\mathrm{~ns})$ & $\tau_{2}(\mathrm{~ns})$ \\
\hline 0 & $0.90 \pm 0.04(15 \%)$ & $3.54 \pm 0.05(85 \%)$ \\
5 & $0.82 \pm 0.06(13 \%)$ & $3.59 \pm 0.06(87 \%)$ \\
10 & $0.73 \pm 0.07(10 \%)$ & $3.55 \pm 0.04(90 \%)$ \\
15 & $0.68 \pm 0.04(10 \%)$ & $3.48 \pm 0.08(90 \%)$ \\
20 & $0.74 \pm 0.05(13 \%)$ & $3.61 \pm 0.07(87 \%)$ \\
25 & $0.66 \pm 0.08(19 \%)$ & $3.45 \pm 0.08(88 \%)$ \\
30 & $0.71 \pm 0.07(15 \%)$ & $3.66 \pm 0.06(85 \%)$ \\
35 & $0.67 \pm 0.04(17 \%)$ & $3.52 \pm 0.04(83 \%)$ \\
40 & $0.66 \pm 0.06(17 \%)$ & $3.51 \pm 0.04(83 \%)$ \\
45 & $0.58 \pm 0.06(17 \%)$ & $3.44 \pm 0.04(83 \%)$ \\
\hline
\end{tabular}


Table B.2. Quenched sensor lifetime by fixing the longer lifetime $\left(\tau_{2}=3.5-3.70 \mathrm{~ns}\right)$ as a constant.

\begin{tabular}{ll}
\hline Network height $(\mu \mathbf{m})$ & $\tau_{1}(\mathrm{~ns})$ \\
\hline 0 & $0.91 \pm 0.05(10 \%)$ \\
5 & $0.89 \pm 0.06(10 \%)$ \\
10 & $0.90 \pm 0.07(10 \%)$ \\
15 & $0.77 \pm 0.04(10 \%)$ \\
20 & $0.76 \pm 0.04(11 \%)$ \\
25 & $0.72 \pm 0.04(10 \%)$ \\
30 & $0.70 \pm 0.07(12 \%)$ \\
35 & $0.69 \pm 0.06(12 \%)$ \\
40 & $0.70 \pm 0.05(10 \%)$ \\
45 & $0.66 \pm 0.07(12 \%)$ \\
\hline
\end{tabular}

Table B.3. Opened sensor lifetime in $R=0.01$ actin network.

\begin{tabular}{ll}
\hline Network z-height $(\mu \mathbf{m})$ & $\tau(\mathrm{ns})$ \\
\hline 0 & $3.79 \pm 0.05$ \\
5 & $3.80 \pm 0.07$ \\
10 & $3.79 \pm 0.05$ \\
15 & $3.82 \pm 0.05$ \\
20 & $3.77 \pm 0.06$ \\
25 & $3.76 \pm 0.08$ \\
30 & $3.71 \pm 0.06$ \\
35 & $3.98 \pm 0.07$ \\
40 & $3.75 \pm 0.05$ \\
45 & $3.78 \pm 0.06$ \\
\hline
\end{tabular}


B.3.2 FLIM: Densely Crosslinked Network $R=0.1$

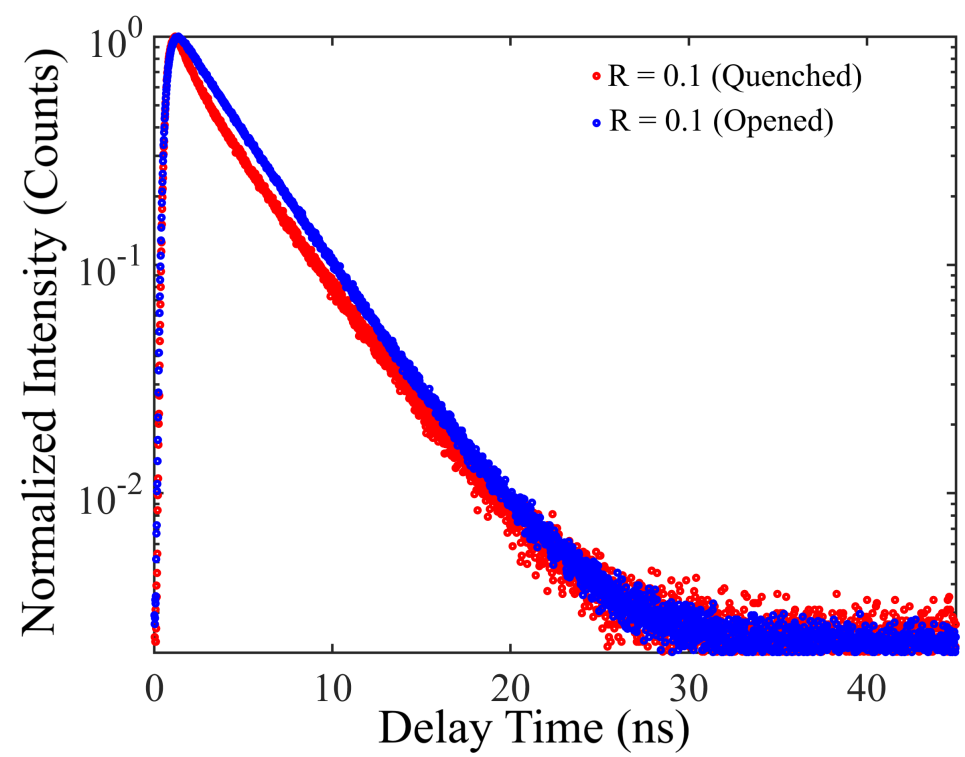

Figure B.6. Lifetime decay curves of DNA sensors in $R=0.1$ actin network with 1:1:1 (F:H:Q) stoichiometry. The red curve denotes the lifetime decay curve of quenched sensors and blue curve represents the lifetime decay of opened sensors. We do not observe any prominent quenching (no bent region) of sensors at this equal stochiometry (1:1:1) in this dense crosslinked network $(R=0.1)$.

Table B.4. Quenched sensor lifetime in $R=0.1$ actin network.

\begin{tabular}{lll}
\hline Network z-height $(\mu \mathbf{m})$ & $\tau_{1}(\mathrm{~ns})$ & $\tau_{2}(\mathrm{~ns})$ \\
\hline 0 & $0.40 \pm 0.06(3 \%)$ & $3.70 \pm 0.06(97 \%)$ \\
1 & $0.60 \pm 0.04(5 \%)$ & $3.70 \pm 0.07(95 \%)$ \\
2 & $0.33 \pm 0.07(2 \%)$ & $3.70 \pm 0.08(98 \%)$ \\
3 & $0.21 \pm 0.06(1 \%)$ & $3.70 \pm 0.05(65 \%)$ \\
4 & $0.17 \pm 0.05(1 \%)$ & $3.70 \pm 0.06(99 \%)$ \\
5 & $0.18 \pm 0.08(1 \%)$ & $3.70 \pm 0.06(99 \%)$ \\
\hline
\end{tabular}


Table B.5. Opened sensor lifetime in $R=0.1$ actin network.

\begin{tabular}{ll}
\hline Network z-height $(\mu \mathbf{m})$ & $\tau(\mathrm{ns})$ \\
\hline 0 & $3.79 \pm 0.05$ \\
1 & $3.79 \pm 0.06$ \\
2 & $3.81 \pm 0.05$ \\
3 & $3.80 \pm 0.07$ \\
4 & $3.81 \pm 0.07$ \\
5 & $3.81 \pm 0.04$ \\
\hline
\end{tabular}

Table B.6. Controls lifetime in $R=0.1$ actin network for closed state.

\begin{tabular}{ll}
\hline Network z-height $(\mu \mathbf{m})$ & $\tau(\mathrm{ns})$ \\
\hline 0 & $3.71 \pm 0.05$ \\
1 & $3.60 \pm 0.07$ \\
2 & $3.67 \pm 0.06$ \\
3 & $3.71 \pm 0.08$ \\
4 & $3.71 \pm 0.04$ \\
5 & $3.70 \pm 0.07$ \\
\hline
\end{tabular}

Table B.7. Controls lifetime in $R=0.1$ actin network for opened state.

\begin{tabular}{ll}
\hline Network z-height $(\mu \mathbf{m})$ & $\tau(\mathrm{ns})$ \\
\hline 0 & $3.95 \pm 0.07$ \\
1 & $3.86 \pm 0.04$ \\
2 & $3.90 \pm 0.06$ \\
3 & $3.92 \pm 0.05$ \\
4 & $3.93 \pm 0.05$ \\
5 & $3.94 \pm 0.07$ \\
\hline
\end{tabular}


B.4 FLIM: DNA sensors in Fibroblasts

a)
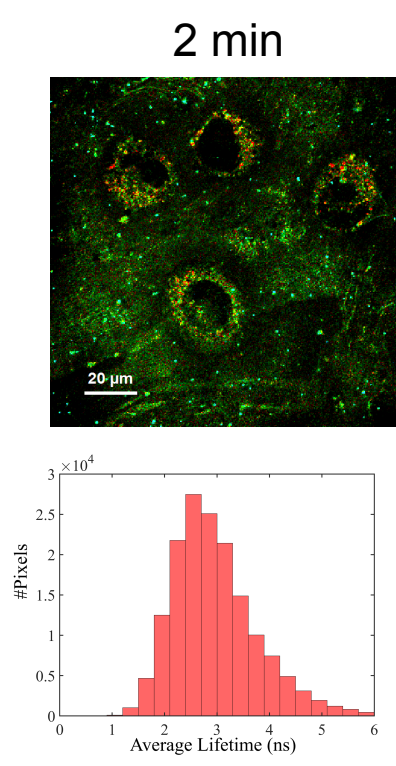

$4 \min$
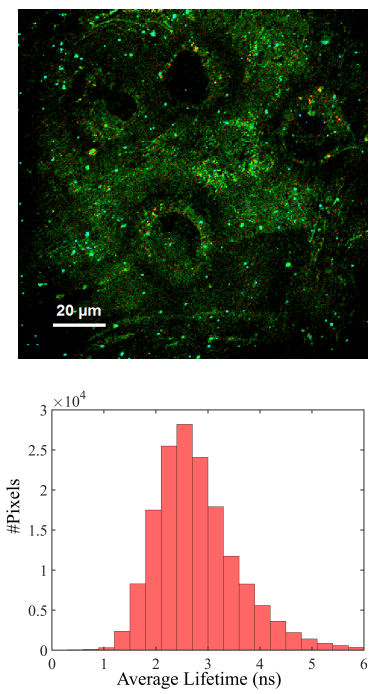

\section{$6 \mathrm{~min}$}
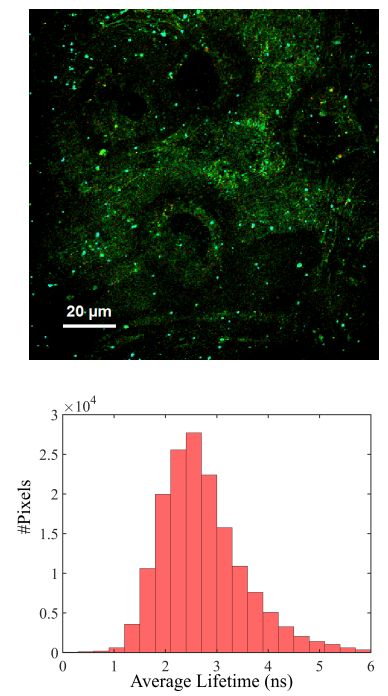

b)
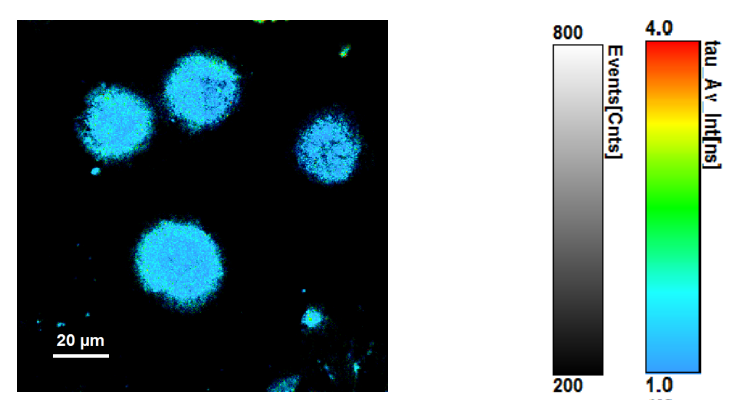

Figure B.7.: DNA sensors in a cluster of fibroblasts. a) A cluster of adherent fibroblasts are imaged at $488 \mathrm{~nm}$ as excitation wavelength for sensors. Their respective lifetimes are represented beneath their images. The sensor lifetimes are between 2 - 4 ns. Lifetimes are analysed framewise. $2 \mathrm{~min}$ indicates frames from $0-2 \mathrm{~min}$ of imaging, $4 \mathrm{~min}$ - frames from $2 \mathrm{~min}$ to $4 \mathrm{~min}, 6 \mathrm{~min}$ frames from $4 \mathrm{~min}$ to $6 \mathrm{~min}$. b) Shows the same cell imaged for actin structures via the lifeact that is tagged to a RFP. 


\section{Bibliography}

[1] Basics of rheology :: Anton Paar Wiki. (cited on page13)

[2] Electroporation and Nucleofector ${ }^{\mathrm{TM}}$ Technology | Lonza. (cited on page 59]

[3] Fluorescence Quantum Yields (QY) and Lifetimes (t) for Alexa Fluor Dyes-Table 1.5. (cited on page 33)

[4] Quick Reference for Confocal Time Resolves Microscopy (FLIM, FRET, FCS). (cited on page 9

[5] Adams, J. C. Roles of fascin in cell adhesion and motility. Current opinion in cell biology 16, 5 (2004), 590-596. (cited on page2)

[6] Alemany, A., Mossa, A., Junier, I., And Ritort, F. Experimental free-energy measurements of kinetic molecular states using fluctuation theorems. Nature Physics 8 (2012), $688 \mathrm{EP}$-. (cited on page 19)

[7] Allersma, M. W., Gittes, F., deCastro, M. J., Stewart, R. J., and Schmidt, C. F. Two-Dimensional Tracking of ncd Motility by Back Focal Plane Interferometry. Biophysical Journal 74, 2 (1998), 1074-1085. (cited on page 19)

[8] Amuasi, H. E., Heussinger, C., Vink, R. L. C., and Zippelius, A. Nonlinear and heterogeneous elasticity of multiply-crosslinked biopolymer networks. New Journal of Physics 17, 8 (2015), 083035. (cited on page 89.

[9] Ashrin, A. Acceleration and Trapping of Particles by Radiation Pressure. Physical Review Letters 26, 4 (1970), 156-159. (cited on page 19)

[10] AshKin, A., AND DzIEDzic, J. M. Optical trapping and manipulation of viruses and bacteria. Science (New York, N.Y.) 235, 4795 (1987), 1517-1520. (cited on page 3 )

[11] Atakhorrami, M., Sulkowska, J. I., Addas, K. M., Koenderink, G. H., Tang, J. X., Levine, A. J., MacKintosh, F. C., AND Schmidt, C. F. Correlated fluctuations of microparticles in viscoelastic solutions: quantitative measurement of material 
properties by microrheology in the presence of optical traps. Physical review. E, Statistical, nonlinear, and soft matter physics 73, $6 \mathrm{Pt} 1$ (2006), 061501. (cited on page 17. 75. and 84

[12] Austen, K., Ringer, P., Mehlich, A., Chrostek-Grashoff, A., Kluger, C., Klingner, C., Sabass, B., Zent, R., Rief, M., and Grashoff, C. Extracellular rigidity sensing by talin isoform-specific mechanical linkages. Nature cell biology 17,12 (2015), 1597-1606. (cited on page4

[13] Bathe, M., Heussinger, C., Claessens, Mireille M. A. E., Bausch, A. R., And FreY, E. Cytoskeletal bundle mechanics. Biophysical Journal 94, 8 (2008), 2955-2964. (cited on page1)

[14] Battle, C. G. Mechanics and Dynamics of the Primary Cilium. PhD thesis, 2013. (cited on page 19

[15] Bausch, A. R., and Kroy, K. A bottom-up approach to cell mechanics. Nature Physics 2, 4 (2006), 231-238. (cited on page68

[16] Bausch, A. R., Ziemann, F., Boulbitch, A. A., Jacobson, K., and Sackmann, E. Local Measurements of Viscoelastic Parameters of Adherent Cell Surfaces by Magnetic Bead Microrheometry. Biophysical Journal 75, 4 (1998), 2038-2049. (cited on page 67

[17] Becker, W., Bergmann, A., Hink, M. A., König, K., Benndorf, K., and Biskup, C. Fluorescence lifetime imaging by time-correlated single-photon counting. Microscopy research and technique 63, 1 (2004), 58-66. (cited on page 11 .

[18] Bershitsky, S. Y., Tsaturyan, A. K., Bershitskaya, O. N., Mashanov, G. I., Brown, P., Burns, R., AND Ferenczi, M. A. Muscle force is generated by myosin heads stereospecifically attached to actin. Nature 388, 6638 (1997), 186-190. (cited on page (1)

[19] Blakely, B. L., Dumelin, C. E., Trappmann, B., McGregor, L. M., Choi, C. K., Anthony, P. C., van Duesterberg, K., BaKer, B. M., Block, S. M., LiU, D. R., AND CHEN, C. S. A DNA-based molecular probe for optically reporting cellular traction forces. Nature methods 11, 12 (2014), 1229-1232. (cited on page 4. 5. and 87)

[20] Blumenfeld, R. Stresses in isostatic granular systems and emergence of force chains. Physical Review Letters 93, 10 (2004), 108301. (cited on page 49)

[21] Brangwynne, C. P., Koenderink, G. H., MacKintosh, F. C., and Weitz, D. A. Nonequilibrium microtubule fluctuations in a model cytoskeleton. Physical Review Letters 100, 11 (2008), 118104. (cited on page1) 
[22] Bray, D., ANd White, J. Cortical flow in animal cells. Science 239, 4842 (1988), 883-888. (cited on page67)

[23] Brenner, M. D., Zhou, R., Conway, D. E., Lanzano, L., Gratton, E., Schwartz, M. A., AND HA, T. Spider Silk Peptide Is a Compact, Linear Nanospring Ideal for Intracellular Tension Sensing. Nano letters 16, 3 (2016), 2096-2102. (cited on page 4)

[24] Bussonnier, M., Carvalho, K., Lemière, J., Joanny, J.-F., Sykes, C., and Betz, T. Mechanical detection of a long-range actin network emanating from a biomimetic cortex. Biophysical Journal 107, 4 (2014), 854-862. (cited on page1)

[25] Bustamante, C., Bryant, Z., And Smith, S. B. Ten years of tension: single-molecule DNA mechanics. Nature 421, 6921 (2003), 423-427. (cited on page 19 )

[26] Chowdhury, F., Li, I. T. S., Ngo, T. T. M., Leslie, B. J., Kim, B. C., Soкoloski, J. E., Weiland, E., Wang, X., Chemla, Y. R., Lohman, T. M., and Ha, T. Defining Single Molecular Forces Required for Notch Activation Using Nano Yoyo. Nano letters 16, 6 (2016), 3892-3897. (cited on page 5 )

[27] Clark, A. G., Dierkes, K., and Paluch, E. K. Monitoring actin cortex thickness in live cells. Biophysical Journal 105, 3 (2013), 570-580. (cited on page2)

[28] Cost, A.-L., Ringer, P., Chrostek-Grashoff, A., and Grashoff, C. How to Measure Molecular Forces in Cells: A Guide to Evaluating Genetically-Encoded FRETBased Tension Sensors. Cellular and molecular bioengineering 8, 1 (2015), 96-105. (cited on page 62

[29] Dasanayake, N. L., Michalski, P. J., and Carlsson, A. E. General mechanism of actomyosin contractility. Physical Review Letters 107, 11 (2011), 118101. (cited on page 1 1)

[30] Discher, D. E., Janmey, P., And Wang, Y.-L. Tissue cells feel and respond to the stiffness of their substrate. Science (New York, N.Y.) 310, 5751 (2005), 1139-1143. (cited on page 1

[31] Dumont, S., And Mitchison, T. J. Force and length in the mitotic spindle. Current biology : CB 19, 17 (2009), R749-61. (cited on page 3 and 87 .

[32] Felix ZÂ"orgiebel. MRToolbox Documentation. (cited on page 18

[33] Fitzpatrick, R., Michieletto, D., Peddireddy, K. R., Hauer, C., Kyrillos, C., Gurmessa, B. J., AND Robertson-Anderson, R. M. Synergistic Interactions Between DNA and Actin Trigger Emergent Viscoelastic Behavior. Physical Review Letters 121, 25 (2018), 257801. (cited on page 84 
[34] Footer, M. J., Kerssemakers, J. W. J., Theriot, J. A., and Dogterom, M. Direct measurement of force generation by actin filament polymerization using an optical trap. Proceedings of the National Academy of Sciences 104, 7 (2007), 2181-2186. (cited on page 3 and 87

[35] Förster, T. Zwischenmolekulare Energiewanderung und Fluoreszenz. Annalen der Physik 437, 1-2 (1948), 55-75. (cited on page 8, 9. and 26)

[36] Frederiksen, D. W., ANd Cunningham, L. W., Eds. Structural and contractile proteins. Pt.B, The contractile apparatus and the cytoskeleton;edited by Dixie W. Frederiksen, Leon W. Cunningham. Methods in Enzymology. Academic Press, New York and London, 1982. (cited on page 93 )

[37] Freikamp, A., Cost, A.-L., and Grashoff, C. The Piconewton Force Awakens: Quantifying Mechanics in Cells. Trends in cell biology 26, 11 (2016), 838-847. (cited on page 4

[38] Gardel, M. L., Nakamura, F., Hartwig, J., Crocker, J. C., Stossel, T. P., and WEITZ, D. A. Stress-dependent elasticity of composite actin networks as a model for cell behavior. Physical Review Letters 96, 8 (2006), 088102. (cited on page1)

[39] Gardel, M. L., Nakamura, F., Hartwig, J. H., Crocker, J. C., Stossel, T. P., and WeITZ, D. A. Prestressed F-actin networks cross-linked by hinged filamins replicate mechanical properties of cells. Proceedings of the National Academy of Sciences 103, 6 (2006), 1762-1767. (cited on page 2 and 68

[40] Gardel, M. L., Shin, J. H., MacKintosh, F. C., Mahadevan, L., Matsudaira, P., AND WEITZ, D. A. Elastic behavior of cross-linked and bundled actin networks. Science (New York, N.Y.) 304, 5675 (2004), 1301-1305. (cited on page 22 and 68)

[41] Gittes, F., And MacKintosh, F. C. Dynamic shear modulus of a semiflexible polymer network. Physical Review E 58, 2 (1998), R1241-R1244. (cited on page 68)

[42] Gittes, F., Mickey, B., Nettleton, J., and Howard, J. Flexural rigidity of microtubules and actin filaments measured from thermal fluctuations in shape. The Journal of Cell Biology 120, 4 (1993), 923-934. (cited on page 68)

[43] Gittes, F., And Schmidt, C. F. Chapter 8 Signals and Noise in Micromechanical Measurements. vol. 55 of Methods in Cell Biology. Elsevier, 1997, pp. 129-156. (cited on page 22]

[44] Gittes, F., Schnurr, B., Olmsted, P. D., MacKintosh, F. C., And Schmidt, C. F. Microscopic Viscoelasticity: Shear Moduli of Soft Materials Determined from Thermal Fluctuations. Physical Review Letters 79, 17 (1997), 3286-3289. (cited on page 22. 68 and 84 
[45] Goktas, M., and Blank, K. G. Molecular Force Sensors: From Fundamental Concepts toward Applications in Cell Biology. Advanced Materials Interfaces 4, 1 (2017), 1600441. (cited on page 5 )

[46] Grashoff, C., Hoffman, B. D., Brenner, M. D., Zhou, R., Parsons, M., Yang, M. T., Mclean, M. A., Sligar, S. G., Chen, C. S., Ha, T., and Schwartz, M. A. Measuring mechanical tension across vinculin reveals regulation of focal adhesion dynamics. Nature 466, 7303 (2010), 263-266. (cited on page 4 and 87)

[47] Grooman, B., Fujiwara, I., Otey, C., and Upadhyaya, A. Morphology and Viscoelasticity of Actin Networks Formed with the Mutually Interacting Crosslinkers: Palladin and Alpha-actinin. PloS one 7, 8 (2012). (cited on page 72 and 84

[48] Hategan, A., Law, R., Kahn, S., and Discher, D. E. Adhesively-Tensed Cell Membranes: Lysis Kinetics and Atomic Force Microscopy Probing. Biophysical Journal 85, 4 (2003), 2746-2759. (cited on page 3 )

[49] Heidemann, K. M., Sharma, A., Rehfeldt, F., Schmidt, C. F., and Wardetzky, M. Elasticity of 3D networks with rigid filaments and compliant crosslinks. Soft matter 11, 2 (2015), 343-354. (cited on page 89.

[50] Hochreiter, B., Garcia, A. P., AND Schmid, J. A. Fluorescent proteins as genetically encoded FRET biosensors in life sciences. Sensors (Basel, Switzerland) 15, 10 (2015), 26281-26314. (cited on page9 9 and 10

[51] Horowitz, P., AND Hill, W. The art of electronics, 2nd ed. ed. 1989. (cited on page 80)

[52] Integrated DNA Technologies. Fluorescence Quenching by Proximal G Bases, 2005 and 2011. (cited on page 44)

[53] Janmey, P. A., Euteneuer, U., Traub, P., and Schliwa, M. Viscoelastic properties of vimentin compared with other filamentous biopolymer networks. The Journal of Cell Biology 113, 1 (1991), 155-160. (cited on page 1)

[54] Janmey, P. A., Georges, P. C., And Hvidt, S. Basic Rheology for Biologists. In Cell Mechanics, vol. 83 of Methods in Cell Biology. Elsevier, 2007, pp. 1-27. (cited on page 13 and 16

[55] Janmey, P. A., Hvidt, S., Käs, J., Lerche, D., Maggs, A., Sackmann, E., Schliwa, M., AND STOSSEL, T. P. The mechanical properties of actin gels. Elastic modulus and filament motions. Journal of Biological Chemistry 269, 51 (1994), 32503-32513. (cited on page 1.59 and 68

[56] Janmey, P. A., ANd Weitz, D. A. Dealing with mechanics: mechanisms of force transduction in cells. Trends in biochemical sciences 29, 7 (2004), 364-370. (cited on page 11 


\section{Appendix $\mid$ Bibliography}

[57] Johansson, M. K. Choosing reporter-quencher pairs for efficient quenching through formation of intramolecular dimers. Methods in molecular biology (Clifton, N.J.) 335 (2006), 17-29. (cited on page 26, 31, and 44 ,

[58] Joost van Mameren. Single molecule mechanics of biopolymers: An optical tweezers study. PhD thesis, 2002. (cited on page 19 and 20)

[59] Koenderink, G. H., Atakhorrami, M., MacKintosh, F. C., and Schmidt, C. F. High-frequency stress relaxation in semiflexible polymer solutions and networks. Physical Review Letters 96, 13 (2006), 138307. (cited on page 68 and 84

[60] Lakowicz, J. R. Principles of fluorescence spectroscopy, 3rd ed. ed. Springer, New York, 2006. (cited on page 7 and 10 )

[61] LARSON, R. G. THE_STRUCTURE_AND_RHEOLOGY_OF_COMPLEX_FL. Oxford University Press, 1999. (cited on page 14 and 17 .

[62] Lee, H., Ferrer, J. M., Nakamura, F., Lang, M. J., and Kamm, R. D. Passive and active microrheology for cross-linked F-actin networks in vitro. Acta biomaterialia 6, 4 (2010), 1207-1218. (cited on page 83

[63] Levine, A. J., AND Lubensky, T. C. Response function of a sphere in a viscoelastic two-fluid medium. Physical review. E, Statistical, nonlinear, and soft matter physics 63, 4 Pt 1 (2001), 041510. (cited on page 17)

[64] Lieleg,, O., Claessens, M. M. A. E., Heussinger, C., Frey, E., and Bausch, A. R. Mechanics of bundled semiflexible polymer networks. Physical Review Letters 99, 8 (2007), 088102. (cited on page2)

[65] Liv, B., Chen, W., Evavold, B. D., and Zhu, C. Accumulation of dynamic catch bonds between TCR and agonist peptide-MHC triggers T cell signaling. Cell 157, 2 (2014), 357-368. (cited on page3)

[66] Liv, Y., Blanchfield, L., Ma, V. P.-Y., Andargachew, R., Galior, K., Liu, Z., Evavold, B., AND SAlaita, K. DNA-based nanoparticle tension sensors reveal that T-cell receptors transmit defined $\mathrm{pN}$ forces to their antigens for enhanced fidelity. Proceedings of the National Academy of Sciences of the United States of America 113, 20 (2016), 5610-5615. (cited on page 5 and 87

[67] Liv, Y., Galion, K., Ma, V. P.-Y., and Salaita, K. Molecular Tension Probes for Imaging Forces at the Cell Surface. Accounts of chemical research 50, 12 (2017), 29152924. (cited on page 5 and 34 )

[68] Loidolt-Krüger, M. STED Microscopy of FRET Pairs. PhD thesis, 2018. (cited on page 10) 
[69] Lorenz, J. S., Schnauss, J., Glaser, M., Sajfutdinow, M., Schuldt, C., Käs, J. A., And Smith, D. M. Synthetic Transient Crosslinks Program the Mechanics of Soft, Biopolymer-Based Materials. Advanced materials (Deerfield Beach, Fla.) 30, 13 (2018), e1706092. (cited on page 70,84 and 85

[70] Los, G. V., Encell, L. P., McDougall, M. G., Hartzell, D. D., Karassina, N., Zimprich, C., Wood, M. G., Learish, R., Ohana, R. F., Urh, M., Simpson, D., MendeZ, J., Zimmerman, K., Otto, P., Vidugiris, G., Zhu, J., Darzins, A., Klaubert, D. H., BulleIt, R. F., AND Wood, K. V. HaloTag: a novel protein labeling technology for cell imaging and protein analysis. ACS chemical biology 3, 6 (2008), 373-382. (cited on page 45 and 87 ,

[71] Ma, V. P.-Y., Liu, Y., Blanchfield, L., Su, H., Evavold, B. D., and Salaita, K. Ratiometric Tension Probes for Mapping Receptor Forces and Clustering at Intermembrane Junctions. Nano letters 16, 7 (2016), 4552-4559. (cited on page5)

[72] Machesky, L. M., Atkinson, S. J., Ampe, C., Vandekerckhove, J., and Pollard, T. D. Purification of a cortical complex containing two unconventional actins from Acanthamoeba by affinity chromatography on profilin-agarose. The Journal of Cell Biology 127, 1 (1994), 107-115. (cited on page2)

[73] MacKintosh, Käs, AND Janmey. Elasticity of semiflexible biopolymer networks. Physical Review Letters 75, 24 (1995), 4425-4428. (cited on page 68)

[74] MacKintosh, F. C., ANd Schmidt, C. F. Active cellular materials. Current opinion in cell biology 22, 1 (2010), 29-35. (cited on page 68

[75] MacLean-Fletcher, S., and Pollard, T. D. Identification of a factor in conventional muscle actin preparations which inhibits actin filament self-association. Biochemical and biophysical research communications 96, 1 (1980), 18-27. (cited on page 93.

[76] Malkin, A. I., And Isayev, A. I. Rheology: Concepts, methods, and applications, 2nd ed. ed. ChemTec Pub, Toronto, 2012. (cited on page13)

[77] Marcel Bremerich. High-bandwidth microrheology of cytoskeletal networks. PhD thesis, 2011. (cited on page 18 and 19

[78] Marras, S. A. E., Kramer, F. R., AND Tyagi, S. Efficiencies of fluorescence resonance energy transfer and contact-mediated quenching in oligonucleotide probes. Nucleic Acids Research 30, 21 (2002), e122. (cited on page 44)

[79] McCall, P. M., MacKintosh, F. C., Kovar, D. R., and Gardel, M. L. Cofilin Drives Rapid Turnover and Fluidization of Entangled F-actin. 2017. (cited on page 84 


\section{Appendix $\quad$ Bibliography}

[80] Meenakshi Prabhune. DNA-based molecular force sensors in cytoskeletal networks and cells. PhD thesis, 2015. (cited on page 25)

[81] Menta, A. D. Single-Molecule Biomechanics with Optical Methods. Science 283, 5408 (1999), 1689-1695. (cited on page 19)

[82] Melak, M., Plessner, M., and Grosse, R. Actin visualization at a glance. Journal of Cell Science 130, 3 (2017), 525-530. (cited on page65)

[83] Meng, F., AND Sachs, F. Orientation-based FRET sensor for real-time imaging of cellular forces. Journal of Cell Science 125, Pt 3 (2012), 743-750. (cited on page 4 and 87)

[84] Meng, F., Suchyna, T. M., AND Sachs, F. A fluorescence energy transfer-based mechanical stress sensor for specific proteins in situ. The FEBS journal 275, 12 (2008), 3072-3087. (cited on page 4 and 87 )

[85] Michelot, A., AND Drubin, D. G. Building distinct actin filament networks in a common cytoplasm. Current biology : CB 21, 14 (2011), R560-9. (cited on page2)

[86] Mira Bovellan. Assembly and composition of the cellular actin cortex. PhD thesis, University College London, London, 2012. (cited on page2 and (3)

[87] MITCHELL, J. R. The Rheology of Gels. Journal of Texture Studies 11, 4 (1980), 315337. (cited on page 13 and 15 )

[88] Mizuno, D., Tardin, C., Schmidt, C. F., and MacKintosh, F. C. Nonequilibrium mechanics of active cytoskeletal networks. Science 315, 5810 (2007), 370-373. (cited on page 1 and 19 .

[89] Morone, N., Fujiwara, T., Murase, K., Kasai, R. S., Ike, H., Yuasa, S., Usukura, J., AND Kusumi, A. Three-dimensional reconstruction of the membrane skeleton at the plasma membrane interface by electron tomography. The Journal of Cell Biology 174, 6 (2006), 851-862. (cited on page 2 )

[90] Morrison, L. E., Halder, T. C., and Stols, L. M. Solution-phase detection of polynucleotides using interacting fluorescent labels and competitive hybridization. Analytical Biochemistry 183, 2 (1989), 231-244. (cited on page 29

[91] Morse, D. C. Viscoelasticity of tightly entangled solutions of semiflexible polymers. Physical Review E 58, 2 (1998), R1237-R1240. (cited on page68)

[92] Munsie, L. N., Caron, N., Desmond, C. R., and Truant, R. Lifeact cannot visualize some forms of stress-induced twisted F-actin. Nature methods 6, 5 (2009), 317. (cited on page 65 
[93] Nazarenko, I., Pires, R., Lowe, B., Obaidy, M., and Rashtchian, A. Effect of primary and secondary structure of oligodeoxyribonucleotides on the fluorescent properties of conjugated dyes. Nucleic Acids Research 30, 9 (2002), 2089-2195. (cited on page 44

[94] Neuman, K. C., And Block, S. M. Optical trapping. The Review of scientific instruments 75, 9 (2004), 2787-2809. (cited on page 19 )

[95] NickLAs, R. B. Measurements of the force produced by the mitotic spindle in anaphase. The Journal of Cell Biology 97, 2 (1983), 542-548. (cited on page 3 and 87 )

[96] Nikon Microscopyu. Basics of FRET Microscopy: Fundamental Principles of Förster Resonance Energy Transfer (FRET) Microscopy with Fluorescent Proteins. (cited on page 9)

[97] Noble, J. E., Wang, L., Cole, K. D., and Gaigalas, A. K. The effect of overhanging nucleotides on fluorescence properties of hybridising oligonucleotides labelled with Alexa-488 and FAM fluorophores. Biophysical chemistry 113, 3 (2005), 255-263. (cited on page 44,

[98] Oliva Saldanha. Tracking Assembly Kinetics of Intermediate Filaments. PhD thesis. (cited on page 2)

[99] Olympus. Fluorescence Resonance Energy Transfer (FRET) Microscopy. (cited on page 10.

[100] Orthaus, S., Buschmann, V., Büller, A., Fore, S., König, M., and Erdmann, R. Quantitative in vivo imaging of molecular distances using FLIM-FRET. (cited on page 11]

[101] Pardee, J. D., And Aspudich, J. [18] Purification of muscle actin. In Structural and contractile proteins. Pt.B, The contractile apparatus and the cytoskeleton;edited by Dixie W. Frederiksen, Leon W. Cunningham, D. W. Frederiksen and L. W. Cunningham, Eds., vol. 85 of Methods in Enzymology. Academic Press, New York and London, 1982, pp. 164-181. (cited on page 93 )

[102] Pelletier, V., Gal, N., Fournier, P., and Kilfoil, M. L. Microrheology of microtubule solutions and actin-microtubule composite networks. Physical Review Letters 102, 18 (2009), 188303. (cited on page 19 .

[103] Qu, P., Chen, X., Zhou, X., LI, X., and ZhaO, X. Fluorescence quenching of TMR by guanosine in oligonucleotides. Science in China Series B: Chemistry 52, 10 (2009), 1653-1659. (cited on page 44 
[104] Renner, S., Bessonov, A., Gerland, U., and Simmel, F. C. Sequence-dependent unfolding kinetics of DNA hairpins studied by nanopore force spectroscopy. Journal of physics. Condensed matter : an Institute of Physics journal 22, 45 (2010), 454119. (cited on page 44

[105] Ridley, A. J., Schwartz, M. A., Burridge, K., Firtel, R. A., Ginsberg, M. H., Borisy, G., PARsons, J. T., AND Horwitz, A. R. Cell migration: integrating signals from front to back. Science (New York, N.Y.) 302, 5651 (2003), 1704-1709. (cited on page 11

[106] Riedl, J., Crevenna, A. H., Kessenbrock, K., Yu, J. H., Neukirchen, D., Bista, M., Bradke, F., Jenne, D., HolaK, T. A., Werb, Z., Sixt, M., and Wedlich-Soldner, R. Lifeact: a versatile marker to visualize F-actin. Nature methods 5, 7 (2008), 605-607. (cited on page 45, 67, and 72

[107] Samaneh Rezvani. Mechanical and biochemical stimulation of suspended cells in a microfluidic device. PhD thesis, 2017. (cited on page 19)

[108] Sanders, T. A., Llagostera, E., And Barna, M. Specialized filopodia direct longrange transport of SHH during vertebrate tissue patterning. Nature 497, 7451 (2013), 628-632. (cited on page65)

[109] Schaap, I. A. T., Carrasco, C., de Pablo, P. J., MacKintosh, F. C., and Schmidt, C. F. Elastic response, buckling, and instability of microtubules under radial indentation. Biophysical Journal 91, 4 (2006), 1521-1531. (cited on page1)

[110] Schlosser, F. Mechanics of suspended cells probed by dual optical traps in a confocal microscope. PhD thesis, Göttingen, June 12th, 2015. (cited on page 24)

[111] Schnurr, B., Gittes, F., MacKintosh, F. C., And Schmidt, C. F. Determining Microscopic Viscoelasticity in Flexible and Semiflexible Polymer Networks from Thermal Fluctuations. Macromolecules 30, 25 (1997), 7781-7792. (cited on page68

[112] Seidel, C. A. M., Schulz, A., And Sauer, M. H. M. Nucleobase-Specific Quenching of Fluorescent Dyes. 1. Nucleobase One-Electron Redox Potentials and Their Correlation with Static and Dynamic Quenching Efficiencies. The Journal of Physical Chemistry 100, 13 (1996), 5541-5553. (cited on page 32 and 44.

[113] Shroff, H., Reinhard, B. M., Siu, M., Agarwal, H., Spakowitz, A., and Liphardt, J. Biocompatible Force Sensor with Optical Readout and Dimensions of $6 \mathrm{~nm} 3$. Nano letters 5, 7 (2005), 1509-1514. (cited on page 5 and 44

[114] Smith, S. B., Finzi, L., ANd Bustamante, C. Direct mechanical measurements of the elasticity of single DNA molecules by using magnetic beads. Science (New York, N.Y.) 258, 5085 (1992), 1122-1126. (cited on page 3 ) 
[115] Soares e Silva, M., Depken, M., Stuhrmann, B., Korsten, M., MacKintosh, F. C., AND KoenderinK, G. H. Active multistage coarsening of actin networks driven by myosin motors. Proceedings of the National Academy of Sciences of the United States of America 108, 23 (2011), 9408-9413. (cited on page 1 )

[116] Storm," Cornelis, Pastore,, Jennifer J., MacKintosh, F. C., Lubensky, T., and JANMEY, P. A. Nonlinear elasticity in biological gels. Nature 435, 7039 (2005), 191194. (cited on page 68)

[117] Stossel, T. P., Condeelis, J., Cooley, L., Hartwig, J. H., Noegel, A., Schleicher, M., AND Shapiro, S. S. Filamins as integrators of cell mechanics and signalling. Nature Reviews. Molecular Cell Biology 2, 2 (2001), 138-145. (cited on page 2 )

[118] Style, R. W., Boltyanskiy, R., German, G. K., Hyland, C., MacMinn, C. W., Mertz, A. F., Wilen, L. A., Xu, Y., AND Dufresne, E. R. Traction force microscopy in physics and biology. Soft matter 10, 23 (2014), 4047-4055. (cited on page 3 )

[119] Svoboda, K., And Block, S. M. Biological applications of optical forces. Annual review of biophysics and biomolecular structure 23 (1994), 247-285. (cited on page 19)

[120] Tan, J. L., Tien, J., Pirone, D. M., Gray, D. S., Bhadriraju, K., and Chen, C. S. Cells lying on a bed of microneedles: an approach to isolate mechanical force. Proceedings of the National Academy of Sciences 100, 4 (2003), 1484-1489. (cited on page 3 )

[121] TAN, Z.-J., AND CHEN, S.-J. Salt dependence of nucleic acid hairpin stability. Biophysical Journal 95, 2 (2008), 738-752. (cited on page 44.

[122] Toyota, T., Head, D. A., Schmidt, C. F., And Mizuno, D. Non-Gaussian athermal fluctuations in active gels. Soft matter 7, 7 (2011), 3234. (cited on page 19 )

[123] Trautmann, S., Buschmann, V., Orthaus, S., Koberling, F., Ortmann, U., and ERdmann, R. Fluorescence Lifetimme Imaging (FLIM) in Confocal Microscopy Applications:An Overview. (cited on page 10 )

[124] Tseng, Y., AND Wirtz, D. Mechanics and Multiple-Particle Tracking Microheterogeneity of a-Actinin-Cross-Linked Actin Filament Networks. Biophysical Journal 81, 3 (2001), 1643-1656. (cited on page1)

[125] Tsukanov, R., Tomov, T. E., Masoud, R., Drory, H., Plavner, N., Liber, M., and NIR, E. Detailed study of DNA hairpin dynamics using single-molecule fluorescence assisted by DNA origami. The Journal of Physical Chemistry. B 117, 40 (2013), 1193211942. (cited on page 4)

[126] Veigel, C., Wang, F., Bartoo, M. L., Sellerrs, J. R., And Molloy, J. E. The gated gait of the processive molecular motor, myosin V. Nature cell biology 4, 1 (2002), 59-65. (cited on page19 
[127] Wachsstock, D. H., Schwartz, W. H., ANd Pollard, T. D. Affinity of alpha-actinin for actin determines the structure and mechanical properties of actin filament gels. Biophysical Journal 65, 1 (1993), 205-214. (cited on page2)

[128] WaHL, M. Time Correlated Single Photon Counting, 2014. (cited on page 11 and 12 )

[129] Wahl, M., and Orthaus-Müller, S. Time Tagged Time-Resolved Fluorescence Data Collection in Life Sciences, 2014. (cited on page 12,

[130] Wan, Z., Chen, X., Chen, H., Ji, Q., Chen, Y., Wang, J., CaO, Y., Wang, F., Lou, J., TANG, Z., AND LiU, W. The activation of IgM- or isotype-switched IgG- and IgE-BCR exhibits distinct mechanical force sensitivity and threshold. eLife 4 (2015). (cited on page 5

[131] WANG, N., Butler, J. P., AND IngBer, D. E. Mechanotransduction across the cell surface and through the cytoskeleton. Science (New York, N.Y.) 260, 5111 (1993), 1124-1127. (cited on page 1 )

[132] WANG, X., AND HA, T. Defining single molecular forces required to activate integrin and notch signaling. Science (New York, N.Y.) 340, 6135 (2013), 991-994. (cited on page 4 and 87

[133] Wang, X., Rahil, Z., LI, I. T. S., Chowdhury, F., Leckband, D. E., Chemla, Y. R., AND HA, T. Constructing modular and universal single molecule tension sensor using protein G to study mechano-sensitive receptors. Scientific reports 6 (2016), 21584. (cited on page 5 )

[134] Ward, S. M. V., Weins, A., Pollak, M. R., and Weitz, D. A. Dynamic Viscoelasticity of Actin Cross-Linked with Wild-Type and Disease-Causing Mutant a-Actinin-4. Biophysical Journal 95, 10 (2008), 4915-4923. (cited on page 66)

[135] Weins, A., Schlondorff, J. S., Nakamura, F., Denker, B. M., Hartwig, J. H., Stossel, T. P., AND PollaK, M. R. Disease-associated mutant a-actinin-4 reveals a mechanism for regulating its F-actin-binding affinity. Proceedings of the National Academy of Sciences 104, 41 (2007), 16080-16085. (cited on page 66

[136] Woodside, M. T., Anthony, P. C., Behnke-Parks, W. M., Larizadeh, K., HerSCHLAG, D., AND BLOCK, S. M. Direct measurement of the full, sequence-dependent folding landscape of a nucleic acid. Science (New York, N.Y.) 314, 5801 (2006), 10011004. (cited on page 44

[137] Woodside, M. T., BehnKe-Parks, W. M., Larizadeh, K., Travers, K., Herschlag, D., AND BLOCK, S. M. Nanomechanical measurements of the sequence-dependent folding landscapes of single nucleic acid hairpins. Proceedings of the National Academy of Sciences 103, 16 (2006), 6190-6195. (cited on page 443 and 44) 
[138] Zhang, Y., Ge, C., Zhu, C., ANd Salaita, K. DNA-based digital tension probes reveal integrin forces during early cell adhesion. Nature Communications 5 (2014), 5167. (cited on page 4, 5, 34, 43,44 and 87

[139] Zribi, O. V., Kyung, H., Golestanian, R., Liverpool, T. B., and Wong, G. C. L. Condensation of DNA-actin polyelectrolyte mixtures driven by ions of different valences. Physical review. E, Statistical, nonlinear, and soft matter physics 73, $3 \mathrm{Pt} 1$ (2006), 031911. (cited on page 84 ) 



\section{List of Figures}

1.1. Cytoskeletal components of a cell. . . . . . . . . . . . . . 2

1.2. Actin structures in an adherent cell . . . . . . . . . . . . . . . 3

1.3. Physical forces at the molecular scale $\ldots \ldots \ldots \ldots \ldots$

2.1. FRET mechanism . . . . . . . . . . . . . . . . . . . 9

2.2. Time correlated single photon counting . . . . . . . . . . . 12

2.3. Shear deformation of an elastic solid and viscous fluid . . . . . . . . . . . 13

2.4. Measuring geometries of a rheometer . . . . . . . . . . . . . 14

2.5. A dynamic experiment illustrating responses of a solid, liquid and vis-

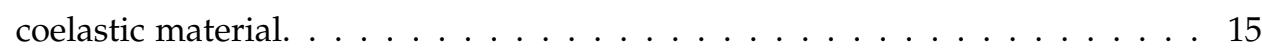

2.6. Detection via back focal plane interferometry . . . . . . . . . . . . . 20

2.7. Calibration of optical trap using the power spectrum of an embedded particle 21

2.8. A scheme of the optical trap set up. . . . . . . . . . . . . . 23

3.1. DNA force sensor design . . . . . . . . . . . . . . . . . 26

3.2. DNA force sensor and controls fluorescence conformation. . . . . . . . . . 27

3.3. Assembly of sensors in DNA buffer. . . . . . . . . . . . . . . . 28

3.4. Opening of hairpins governed by their stem energies. . . . . . . . . . . . . 29

3.5. Fluorescence of DNA force sensor versus controls in a spectrometer. . . . . . 30

3.6. Structural quenching from strands. . . . . . . . . . . . . . 31

3.7. Lifetime decay curves of different strands. . . . . . . . . . . . . . 33

3.8. Lifetime decay curves of sensors with decreasing $\mathrm{F}$ strand concentration. . . 34

3.9. Total photon counts of quenched sensors for decreasing concentration of $F$

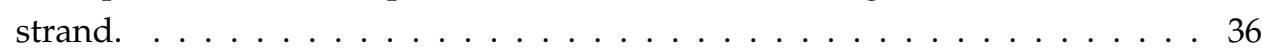

3.10. Quenched and opened sensors lifetime decay curves in DNA and actin

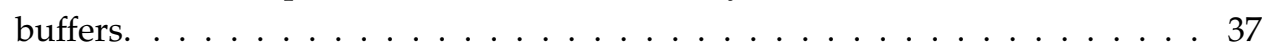

3.11. Lifetime decay curves of controls. . . . . . . . . . . . . . . 38

3.12. PCR annealed sensors and controls lifetime measurements. . . . . . . . . . . 39

3.13. PCR and room temperature annealed sensors . . . . . . . . . . . . 41

3.14. Distribution of quenched and fluorescence lifetime of sensors. . . . . . . . . 42 
3.15. FRET efficiency of DNA sensors $\ldots \ldots \ldots \ldots$. . . . . . . . 42

4.1. DNA sensor attachment to actin network. . . . . . . . . . . . . 46

4.2. Working of sensors crosslinked to actin . . . . . . . . . . . . . 47

4.3. Fluorescence of DNA sensors versus controls when crosslinked to actin . . . 48

4.4. Intensity of quenched and fluorescent sensors across actin network. . . . . . 49

4.5. Controls fluorescence intensity across actin network. . . . . . . . . . . . 50

4.6. Fluorescence lifetime decay curves of $R=0.01$ actin-DNA sensor network in $0.5: 1: 1$ stoichiometry. . . . . . . . . . . . . . . . . . . 52

4.7. Lifetime decay curves of $R=0.1$ actin-DNA sensor network in $0.5: 1: 1$ (F:H:Q)

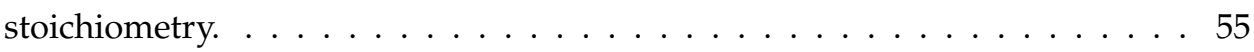

4.8. DNA sensor and controls lifetime decay curves for $R=0.1$ actin-sensor net-

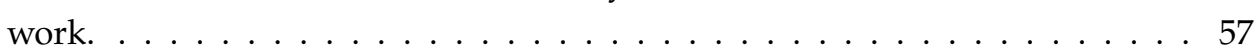

4.9. Homogenous quenching of sensors in $R=0.1$ actin-DNA sensor network at 0.5:1:1 (F:H:Q) stoichiometry. . . . . . . . . . . . . . . . 58

4.10. Lifetimes of sensor and controls in $R=0.1$ actin-DNA sensor network for 0.5:1:1 (F:H:Q) stoichiometry. . . . . . . . . . . . . . . . . . . 58

4.11. DNA sensors inside live 3T3 fibroblasts. . . . . . . . . . . . . . . 60

4.12. Controls inside adherent $3 \mathrm{~T} 3$ fibroblasts. . . . . . . . . . . . . . 63

4.13. The lifetime of DNA sensors versus controls in $3 \mathrm{~T} 3$ fibroblasts. . . . . . . . . 64

5.1. Gelation kinetics of actin and actin-DNA sensor networks . . . . . . . . . 70

5.2. Bulk frequency response of actin, actin-DNA sensor networks in a shear

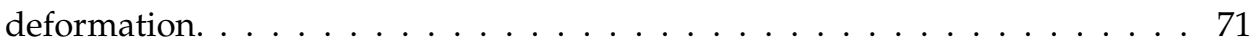

5.3. Microstructure of equal stoichiometry (1:1:1) actin-sensor networks. . . . . 73

5.4. Microstructure of half stoichiometry (0.5:1:1) actin-DNA sensor networks. . . 74

5.5. The power spectrum of bead in water and actin at varying laser powers. . . 76

5.6. The viscosity of water at different laser powers. . . . . . . . . . . . 77

5.7. The storage modulus of actin at various laser powers. . . . . . . . . . 78

5.8. The loss modulus of actin at varying laser powers. . . . . . . . . . . . 79

5.9. Water PSD at various laser powers for different sampling frequency. . . . . . 80

5.10. Power Spectra of water, actin and actin-DNA sensor network. . . . . . . . . . 81

5.11. Frequency dependent $G^{\prime}$ of actin and actin-DNA sensor network. . . . . . . 82

5.12. Frequency dependent $G^{\prime \prime}$ of actin, actin-DNA sensor networks. . . . . . . . 83

A.1. Sensor preparation . . . . . . . . . . . . . . . . . . . . . . 97

B.1. Fluorescence of DNA force sensor versus controls when crosslinked to actin

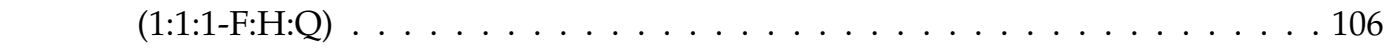

B.2. Scheme of a partially assembled sensor . . . . . . . . . . . . . . 106

B.3. Quenched and fluorescence intensity of sensors in actin network 1:1:1-F:H:Q 107

B.4. Controls fluorescence intensity in actin network for 1:1:1-F:H:Q . . . . . . 107 
B.5. Lifetime decay curves of $R=0.01$ actin-sensor network 1:1:1 (F:H:Q) sto-

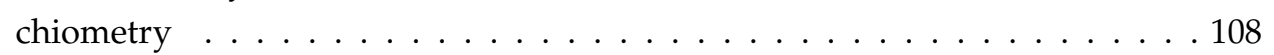

B.6. Lifetime decay curves of DNA sensors in $R=0.1$ actin network with 1:1:1

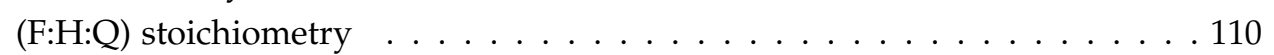

B.7. DNA sensors in a cluster of fibroblasts . . . . . . . . . . . . . . 112 



\section{List of Tables}

3.1. Lifetime of different sensor strands . . . . . . . . . . . . . . . . . . 34

3.2. Lifetime of quenched sensors (FHQ) with decreasing F strand concentration 35

3.3. Lifetime of quenched sensors (FHQ) with decreasing F strands when $\tau_{2}=\mathrm{a}$

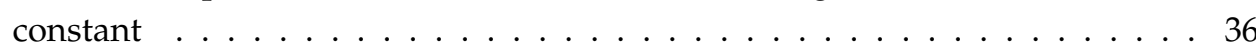

3.4. Lifetime of DNA sensors when quenched $(\mathrm{F}+\mathrm{H}+\mathrm{Q})$ and opened $(\mathrm{F}+\mathrm{H}+\mathrm{Q}+\mathrm{C})$ in DNA and actin buffer . . . . . . . . . . . . . . . . 37

3.5. Lifetime of controls in DNA and actin buffer . . . . . . . . . . . 38

3.6. Lifetimes of annealed DNA sensors and annealed controls . . . . . . . . . . 40

4.1. Quenched sensors lifetime and their amplitude (in percentages) in $R=0.01$ actin network at different z-positions $\ldots \ldots \ldots \ldots \ldots$

4.2. Quenched sensors lifetime and amplitude (in percentages) in $R=0.01$ actin network when the longer lifetime $\left(\tau_{2}=3.5-3.70 \mathrm{~ns}\right)$ is held as a constant . . . 53

4.3. Opened sensors lifetime in $R=0.01$ actin network . . . . . . . . . . . 54

4.4. Quenched sensor lifetime and amplitude (in percentages) in $R=0.1$ actin

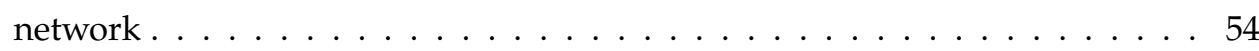

4.5. Opened sensors lifetime in $R=0.1$ actin network . . . . . . . . . . . 56

4.6. Controls lifetime in $R=0.1$ actin network for their closed state . . . . . . . . . 56

4.7. Controls lifetime in $R=0.1$ actin network for their opened state . . . . . . . 57

5.1. Networks and crosslinking ratios . . . . . . . . . . . . . . . . . . 69

A.1. Actin polymerization buffer - Polymix 10X . . . . . . . . . . . . . . . 96

A.2. DNA hybridization buffer $\ldots \ldots \ldots \ldots$. . . . . . . . . . . . . 96

A.3. DNA oligos sequence. . . . . . . . . . . . . . . . . . . . . . 96

A.4. PCR annealing of DNA strands . . . . . . . . . . . . . . . . . 96

B.1. Quenched sensor lifetime across actin network $R=0.01$. . . . . . . . . . . . . 108

B.2. Quenched sensor lifetime by fixing the longer lifetime $\left(\tau_{2}=3.5-3.70 \mathrm{~ns}\right)$ as a constant. . . . . . . . . . . . . . . . . . . . . . . 109

B.3. Opened sensor lifetime in $R=0.01$ actin network. . . . . . . . . . . . . 109 
Appendix $\mid$ List of Tables

B.4. Quenched sensor lifetime in $R=0.1$ actin network. . . . . . . . . . . . . . . . 110

B.5. Opened sensor lifetime in $R=0.1$ actin network. . . . . . . . . . . . . . . . . . . . . . . . . 111

B.6. Controls lifetime in $R=0.1$ actin network for closed state. . . . . . . . . . . . 111

B.7. Controls lifetime in $R=0.1$ actin network for opened state. . . . . . . . . . 111 



\section{Abbreviations}

\begin{tabular}{|c|c|}
\hline ABPs & : Actin binding proteins \\
\hline AFM & : Atomic force microscopy \\
\hline BFP & : Back focal plane \\
\hline $\mathrm{BP}$ & : Base pair \\
\hline C & : Complementary strand \\
\hline CCD & : Charge-Coupled Device \\
\hline $\mathrm{CP}$ & : Cone plate \\
\hline DNA & : Deoxy ribonucleic acid \\
\hline $\mathrm{F}$ & : Fluorophore \\
\hline F-actin & : Filamentous actin \\
\hline FDT & : Fluctuation dissapiation theorem \\
\hline FLIM & : Fluorescence lifetime imaging \\
\hline$f_{N y q}$ & : Nyquist Frequency \\
\hline FRET & : Förster resonance energy transfer \\
\hline$f_{s}$ & : Sampling frequency \\
\hline G-Actin & : Globular actin \\
\hline GFP & : Green Fluorescent Protein \\
\hline $\mathrm{H}$ & : Hairpin \\
\hline KCL & : Potassium Chloride \\
\hline $1_{c}$ & : Contour length \\
\hline $1_{p}$ & : Persistence length \\
\hline MBs & : Molecular Beacons \\
\hline MFSs & : Molecular Force Sensors \\
\hline NA & : Numerical Aperture \\
\hline $\mathrm{nN}$ & : nanoNewton \\
\hline ns & : nanoseconds \\
\hline OD & : Optical Density \\
\hline OT & : Optical Trap \\
\hline PAGE & : Polyacrylamide Gel Electrophoresis \\
\hline PBS & : Phosphate buffer saline \\
\hline PCR (buffers) & : Polymerase Chain Reaction \\
\hline PMR & : Passive microrheology \\
\hline PMT & : Photomultiplier tube \\
\hline
\end{tabular}




$\begin{array}{ll}\text { pN } & \text { : picoNewton } \\ \text { PP } & \text { : Parallel Plate } \\ \text { Q } & \text { : Quencher } \\ \text { QPD / std } & \text { : Quadrant photodiode } \\ \text { RFP } & \text { : Red fluorescent protein } \\ \text { rpm } & \text { : rotations per minute } \\ \text { RT } & \text { : } 75 \text { room temperature } \\ \text { TCSPC } & \text { : Time Correlated Single Photon Counting } \\ \text { TFM } & \text { : Traction force microscopy } \\ \text { TGT } & \text { : Tension Gauge Tether } \\ \text { TS } & : \text { Tension Sensor } \\ \text { WLC } & \text { : Worm like Chain }\end{array}$




\section{Acknowledgments}

First and foremost, I would like to thank my supervisors Christoph F. Schmidt and Florian Rehfeldt. Their guidance to take the right scientific steps, immense patience, dedicated weekly discussions and strong support has led to a success of this project. They motivated me from the beginning to attend many conferences, workshops which gave me the opportunity to learn about the topic deeper. It helped me in staying updated with the international community and to avoid many difficulties. Thank you for believing in me, giving me a freedom and independence which helped me to learn new techniques that was vital for this work. I thank you both for having me start from scratch in biophysics and helping me grow thus far. It felt home to come back after my master thesis to the same group.

I would also like to thank my thesis committee members, Max Wardetzky and Fred Wouters. Their scientific advice, technical expertise, timely direction to strike off on the right path of the project, suggestions which kept me on track for the project goal was an invaluable help that I received during these years. Without your advise on how to lead a project as a graduate student, I would have been lost. It helped me to achieve what was possible. I am glad to have had you both as my thesis advisors.

A substantial part of this thesis is the fantastic collaboration work with Arindam Gosh in the lab of Jörg Enderlein. I thank Jörg Enderlein for his excellent scientific expertise and his appreciating words. Arindam, I thank you very much for your amazing dedication in this work, long overnight measurements and analysis, untiring efforts and for your unmatched instrumental expertise. You were ever present to help and teach. I definitely learnt what collaboration and networking can bring forth to science. Eventually I enjoyed our Indian food and political discussions.

The insight provided by imaging experts Jörg Enderlein and Fred Wouters has contributed to a deeper understanding of this work. It has been enriching and rewarding to be able to understand this project at a fundamentally deeper level. Dieter Klopfenstein, I thank you for your all your inspiring scientific ideas, suggestions for this work during the group seminars, for fixing any issue that I came up to you for and for the annual wine and cheese evenings that you hosted. It was a memorable part of my graduate life.

A lab isn't lively without colleagues of the institute. I was happy to spend these years with the these incredible colleagues around. Alok, André, Miquel (Mike), Chris Battle, Ulrich, Alice, Meenakhsi aka Minu, Adam, Paula, Kai, Volker, Florian Schlosser, Charlotte Willms, Nikta and Nuria thanks for making me feel comfortable in a new place when I started in the lab as a master student. The new members definitely made graduate years 
more fun and an active vibrant atmosphere. I thank Renata, Jianguo, Kengo, Amna, Octavio, Chonglin, Sufi, Samaneh, Gabriela, Galina, Carina, Achintya, Heidi, Lara, Daniel, Constantin, Behzhad for this.

I am grateful to our technical assistants Kerstin von Roden, Ulrike Schulz, Tanja Gall. Thanks for all the underlying important part of this project, the technical experiments from protein purifications (actin from Kerstin and lifeact-halotag from Ulrike), to molecular cloning to running of gels, the biochemistry work etc. Without this sound expertise, it would not have been possible. I thank Nicole Rehbein, for her ever smiling welcoming approach in any of the administrative issues concerned over these years.

I thank my funding agency SFB 755 A3 and my graduate program Göttingen Graduate Centre for Neurosciences, Biophysics and Molecular Biosciences (GGNB) for supporting me financially.

Time spent together with friends outside lab made the years fly by, gave times to laugh, relax and enjoy the community feeling of being home. I thank Samane, Shyam (for his delicious Indian food), Kishore, Andrew, Anupa, Kruttika for this. Samane, for being a wonderful flatmate, a best friend over the years who was there through happy and tough times. You showed me that things are not stressed out as they are and that there is always an easy way. I thank Nina for being there as a wonderful close friend at all times, no words. You were there standing with me through tough days, thank you for schönes Wochenende, Feierabend and for helping me approach problems pragmatically.

Helge who supported me over the many years and for my growth in every way, thank you. Sankar for motivating me to not give up and suggesting me the right tips at the right time. Sumanth you have stood beside me from our undergraduate days, thank you for your support and encouragement from India to Germany. Lara and Heidi you both made my last months happier. I owe you both Indian curries for your extensive corrections on my thesis. Thank you very much. Tridev, I admire you for who you have been during my most difficult phase. Thanks for your solid support and making these days light, enjoyable and funny. If I have missed anyone, I thank you for being a part of this journey.

Finally, I want to thank my dear parents and siblings. They never forgot to call me every week and made sure I still felt embraced as part of the family though miles apart. I thank my parents, Mary Jemima Samuel and P. Jayachandran for their unconditional love, constant encouragement, their support for what I had chosen to do, their guidance during the thesis and for their scientific support. I am proud to have you as my parents. 


\section{Affidavit}

I herewith declare that I have produced this dissertation without the prohibited assistance of third parties and without making use of aids other than those specified; notions taken over directly or indirectly from other sources have been identified as such. This dissertation has not previously been presented in identical or similar form to any other German or foreign examination board.

This thesis work was conducted from November 2015 to July 2019 under the supervision of Prof. Dr. Christoph F. Schmidt and Dr. Florian Rehfeldt at the Third Institute of Physics - Biophysics.

Christina Jayachandran

Göttingen, July 2019 